Supporting Information

for

\title{
Photocatalytic (Het)Arylation of $\mathrm{C}\left(\mathrm{sp}^{3}\right)-\mathrm{H}$ Bonds with Carbon Nitride
}

Saikat Das, ${ }^{a}$ Kathiravan Murugesan, ${ }^{a}$ Gonzalo J. Villegas Rodríguez, ${ }^{a}$ Jaspreet Kaur, ${ }^{a}$ Joshua.

P. Barham, ${ }^{a}$ Aleksandr Savateev, ${ }^{b}$ Markus Antonietti ${ }^{b}$ and Burkhard König*a

${ }^{a}$ Fakultät für Chemie und Pharmazie, Universität Regensburg, 93040 Regensburg, Germany

${ }^{b}$ Department of Colloid Chemistry, Max-Planck Institute of Colloids and Interfaces, Research

Campus Golm, 14424 Potsdam, Germany

*Correspondence: burkhard.koenig@ur.de 


\section{Table of Contents}

1. General Information S1

2. Characterization of fresh mpg-CN photocatalyst S3

3. Description of general synthetic procedures $\quad$ S9

$\begin{array}{lll}3.1 & \text { Synthesis of mpg-CN photocatalyst } & \text { S9 }\end{array}$

3.2 General procedure 1 (GP1) for Aryl Bromides $\quad$ S9

3.3 General procedure 2(GP2) for Aryl Chlorides $\quad$ S10

3.4 General procedure 3 (GP3) for Heteroaryl Halides $\quad$ S10

3.5 General Procedure $4(\mathrm{GP} 4)$ for $\mathrm{C}(\mathrm{sp} 3)-\mathrm{H}$ arylation of amides $\quad$ S11

3.6 General Procedure 5 (GP5) for gram scale synthesis $\quad$ S12

4. Optimization Studies $\quad$ S13

$\begin{array}{llr}\text { 5. Scope and analytical data } & \text { S16 }\end{array}$

6. Characterization of recovered mpg-CN photocatalyst S55

$\begin{array}{llr}\text { 7. Mechanistic Considerations } & \text { S64 }\end{array}$

8. Kinetic Studies and investigation of scalability in Flow S66

$\begin{array}{llr}\text { 9. Supporting references } & \text { S79 }\end{array}$

10. NMR Spectra $\quad$ S81 


\section{General information}

All reactions were carried out with dry solvents unless otherwise stated. Dry nitrogen was used as inert gas atmosphere. All solvents and commercially available reagents were purchased as reagent grade or at the highest commercial quality and used without further purification, unless otherwise stated. Thin-layer chromatography was performed using silica gel plates 60 F254: Visualization was accomplished with short wavelength UV light $(254 \mathrm{~nm})$ and near UV light $(366 \mathrm{~nm})$ sources. Standard flash chromatography was performed on a Biotage ${ }^{\circledR}$ Isolera ${ }^{\mathrm{TM}}$ Spektra system automated with high performance flash purification system using either a prepacked Biotage $\left(\right.$ SNAP Ultra HP-Sphere ${ }^{\mathrm{TM}}$ silica gel of particle size $25 \mu \mathrm{m}$ or silica gel $60 \mathrm{M}$ (particle size 40-63 $\mu \mathrm{m}, 230-440$ mesh, Merck). Photocatalytic reactions were performed using TAK 120 photoreactor equipped with blue LEDs (OSRAM OSLON® SSL 80 GD CS8PM1.14 LEDs $(\lambda=451 \mathrm{~nm}( \pm 15 \mathrm{~nm}), 5 \mathrm{~W}$ optical power), unless stated otherwise. The optical power of LEDs was determined using FieldMaxII-TOTM laser power meter equipped with PM3 sensor. GC measurements were performed on a GC 7890 from Agilent Technologies. Data acquisition and evaluation was done with Agilent Chem Station Rev.C.01.04. GC-MS measurements were performed on a 7890A GC system from Agilent Technologies with an Agilent 5975 MSD Detector. Data acquisition and evaluation was done with MSD Chem Station E.02.02.1431. A capillary column HP-5MS/30 m x $0.25 \mathrm{~mm} / 0.25 \mu \mathrm{M}$ film and helium as carrier gas (flow rate of $1 \mathrm{~mL} / \mathrm{min}$ ) were used. The injector temperature (split injection: 40:1 split) was $280{ }^{\circ} \mathrm{C}$, detection temperature $300{ }^{\circ} \mathrm{C}$ (FID). GC measurements were made and investigated via integration of the signal obtained. The GC oven temperature program was adjusted as follows: initial temperature $40{ }^{\circ} \mathrm{C}$ was kept for 3 minutes, the temperature was increased at a rate of $15{ }^{\circ} \mathrm{C} / \mathrm{min}$ over a period of 16 minutes until $280{ }^{\circ} \mathrm{C}$ was reached and kept for 5 minutes, the temperature was again increased at a rate of $25^{\circ} \mathrm{C} / \mathrm{min}$ over a period of 48 seconds until the final temperature $\left(300^{\circ} \mathrm{C}\right)$ was reached and kept for 5 minutes. 1,4-dimethoxy benzene was used as an internal standard. All NMR spectra were measured at room temperature using a Bruker Avance $300\left(300 \mathrm{MHz}\right.$ for ${ }^{1} \mathrm{H}, 75 \mathrm{MHz}$ for ${ }^{13} \mathrm{C}, 282 \mathrm{MHz}$ for ${ }^{19} \mathrm{~F}$ ) or a Bruker Avance $400\left(400 \mathrm{MHz}\right.$ for ${ }^{1} \mathrm{H}, 101 \mathrm{MHz}$ for ${ }^{13} \mathrm{C}, 376 \mathrm{MHz}$ for ${ }^{19} \mathrm{~F}$ ) NMR spectrometer in $\mathrm{CDCl}_{3}$, DMSO-d6, and MeOH-d4 solutions with internal solvent signals (for ${ }^{1} \mathrm{H}$ and ${ }^{13} \mathrm{C}$ ) as reference (7.26, 77.2 for $\mathrm{CDCl}_{3}, 2.50$ and 39.5 for DMSO-d6, and 3.31, 49.0 for $\mathrm{MeOH}-\mathrm{d} 4$ ). All chemical shifts are reported in $\delta$-scale as parts per million [ppm] (multiplicity, coupling constant $\mathrm{J}$, number of protons) relative to the solvent residual peaks as the internal standard. Coupling constants $\mathrm{J}$ are given in Hertz $[\mathrm{Hz}]$. Abbreviations used for signal multiplicity: ${ }^{1} \mathrm{H}$ NMR: $\mathrm{b}=$ broad, $\mathrm{s}=$ singlet, $\mathrm{d}=$ doublet, $\mathrm{t}=$ triplet, $\mathrm{q}=$ quartet, hept $=$ heptet $\mathrm{dd}=$ doublet of 
doublets, $\mathrm{dt}=$ doublet of triplets, $\mathrm{dq}=$ doublet of quartets, and $\mathrm{m}=$ multiplet; ${ }^{13} \mathrm{C}-\mathrm{NMR}:(+)=$ primary/tertiary, $(-)=$ secondary, $(\mathrm{Cq})=$ quaternary carbon. Most of the characterized compounds gave double signals in ${ }^{1} \mathrm{H},{ }^{13} \mathrm{C} N \mathrm{NM}$ and ${ }^{19} \mathrm{~F} N \mathrm{NR}$, this is due to the rotamer nature of the amide compounds. HRMS (high resolution mass spectra) and LRMS (low resolution mass spectra) were measured at the Central Analytical Laboratory of the University of Regensburg. These mass spectra were recorded on a Finnigan MAT 95, Thermo Quest Finnigan TSQ 7000, Finnigan MAT SSQ 710 A or an Agilent Q-TOF 6540 UHD instrument. Powder XRay diffraction (PXRD) patterns have been acquired using Bruker D8 diffractometer using radiation from $\mathrm{Cu} \mathrm{K \alpha}(\lambda=1.54060 \mathrm{~nm})$ equipped with scintillation counter detector in the range of $2 \theta 3-60^{\circ}$ with step size $0.05^{\circ}$ and time per step $4 \mathrm{~s}$. Scanning electron microscopy (SEM) and Energy Dispersive X-Ray Analysis (EDX) have been performed using JSM-7500F (JEOL) scanning electron microscope at accelerating voltage $3 \mathrm{kV}$. Inductively coupled plasma optical emission spectrometry (ICP-OES) (Optima 8000; Perkin Elmer) was used to analyze Ni content in the samples. X-ray photoelectron spectroscopy (XPS) analysis was carried out on a Thermo Fisher Scientific ESCALAB spectrometer with aluminum K $\alpha$ radiation. Data was processed using Origin 2020b. Shirley's background has been subtracted prior deconvolution of the peaks. Fourier-transform infrared spectra (ATR FT-IR) have been acquired in the range of wavelengths $550-4000 \mathrm{~cm}^{-1}$ using Nicolet iS5 equipped with attenuated total reflectance module iD5. Nitrogen sorption by the materials has been conducted at 77K using Quantasorb SI station. Prior measurements samples were degassed at $150^{\circ} \mathrm{C}$ in vacuum $(<0.2 \mathrm{mbar})$ for $15 \mathrm{~h}$. Relative pressure range (P/P0) from 0.05 to 0.3 was used to calculate surface area of the materials employing Brunauer-Emmett-Teller (BET) theory model in Quantachrome ${ }^{\circledR}$ QuadraWin $^{\mathrm{TM}}$ V.5.11 software. N2 at $77 \mathrm{~K}$ on carbon (slit/cylindric/sphere pores, quenched solid density functional theory (QSDFT), adsorption branch kernel) has been chosen to analyze pore diameter distribution. Diffuse-reflectance UV-vis (DRUV-vis) absorption spectra of the samples in solid state has been acquired using Shimadzu UV-2600 equipped with integrating sphere. Room temperature steady-state photoluminescence spectra of the samples upon excitation with $365 \mathrm{~nm}$, have been acquired using Jasco FP-8300 spectrometer. Transmission electron microscopy (TEM) images were obtained on a double-corrected JEOL ARM200 at an acceleration voltage of $80 \mathrm{kV}$ and an emission of $10 \mu \mathrm{A}$. 


\section{Characterization of fresh mpg-CN photocatalyst}

The structure of the prepared mpg-CN has been confirmed by a series techniques and characterization results are in agreement with the reported earlier data. ${ }^{1}$ EDX elemental composition revealed $\mathrm{C} / \mathrm{N}$ ratio of 0.58 (Table $\mathbf{S 1}$ ), which is lower than expected for melon (i.e. 0.75 ) and is explained by the presence of terminal amino groups in the structure of mpg$\mathrm{CN}$ due to incomplete condensation of the precursor and partially hydroxylated surface. In agreement with this conclusion is FT-IR spectrum (Figure S1). A broad peak with maximum at $3180 \mathrm{~cm}^{-1}$ was assigned to $\mathrm{N}-\mathrm{H}$ and hydrogen-bonded $\mathrm{O}-\mathrm{H}$ stretch; a peak at $2180 \mathrm{~cm}^{-1}$ to nitrile stretch; a broad band between $1000-1650 \mathrm{~cm}^{-1}$ is assigned to vibrations of $\mathrm{CN}$ heterocycles. The peak at $805 \mathrm{~cm}^{-1}$ is assigned to deformation vibration of tri-s-triazine system. In addition, a peak at $1628 \mathrm{~cm}^{-1}$ was assigned to primary amine $\mathrm{N}-\mathrm{H}$ bend; a peak at $1561 \mathrm{~cm}^{-}$ ${ }^{1}$ to secondary amine $\mathrm{N}-\mathrm{H}$ bend; and peaks at $1230,1308,1398 \mathrm{~cm}^{-1}$ to $\mathrm{C}-\mathrm{O}$ stretch.

Table S1. EDX elemental analysis.

\begin{tabular}{|c|c|c|}
\hline C wt. \% & N wt. \% & O wt. \% \\
\hline $36 \pm 1.3$ & $62 \pm 1.2$ & $2 \pm 0.4$ \\
\hline
\end{tabular}

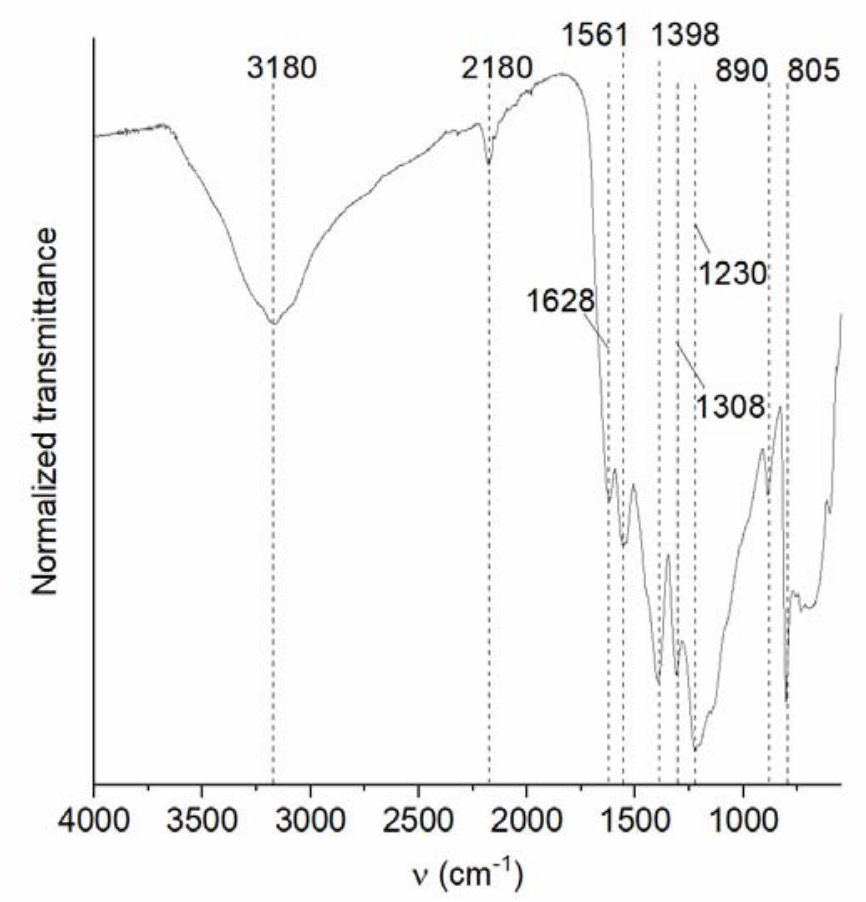

Figure S1. FT-IR spectrum of mpg-CN. 
Given that penetration depth in XPS is limited to $\sim 5 \mathrm{~nm}$, higher oxygen content $(8.3$ at. \%) determined by this technique compared to EDX $(2 \pm 0.4 \%)$, confirmed that oxygen is located mainly on the surface of the material (Table S2). Deconvolution of high-resolution X-Ray photoelectron spectrum of $\mathrm{C} 1 \mathrm{~s}$ confirmed existence of this element in three chemical states (Figure S2). The peak with maximum at $288.3 \mathrm{eV}$ was assigned to carbon coordinated to nitrogen $\left(\mathrm{CN}_{3}\right)$ (Figure $\mathbf{S 2}$ and S3), ${ }^{2,3}$ peak at $286.2 \mathrm{eV}$ to carbon bound to oxygen ${ }^{4,5}$ and peak at $284.7 \mathrm{eV}$ to adventitious carbon. ${ }^{2,4,3}$ Deconvolution of high-resolution X-Ray photoelectron spectrum of $\mathrm{N} 1 \mathrm{~s}$ revealed the following chemical states: peak at $398.8 \mathrm{eV}$ was assigned to dicoordinated nitrogen of $\mathrm{C}=\mathrm{N}-\mathrm{C}$ moieties in tri-s-triazine (Figure S3 and S4), ${ }^{2,5,6}$ peak at 400.1 $\mathrm{eV}$ to the central nitrogen atom of tri-s-triazine, ${ }^{2,6,3}$ peak at $401.3 \mathrm{eV}$ to secondary and primary amino-groups in mpg-CN due to incomplete condensation, ${ }^{2,7,3}$ and a broad peak of low intensity at $404.8 \mathrm{eV}$ to $\pi-\pi^{*}$ satellite or $\pi$-excitation that is indicative for conjugated aromatic structures. ${ }^{8,9}$ Deconvolution of high-resolution X-Ray photoelectron spectrum of O 1s revealed oxygen in two chemical states: the peak at $532.0 \mathrm{eV}$ is assigned to oxygen bound to carbon (Figure S5), ${ }^{2}$ while the peak at $533.4 \mathrm{eV}$ to water absorbed in mpg-CN. ${ }^{2}$

Table S2. XPS elemental analysis.

\begin{tabular}{|c|c|c|}
\hline C at. $\%$ & N at. $\%$ & O at. $\%$ \\
\hline 29.1 & 64.8 & 6.1 \\
\hline
\end{tabular}

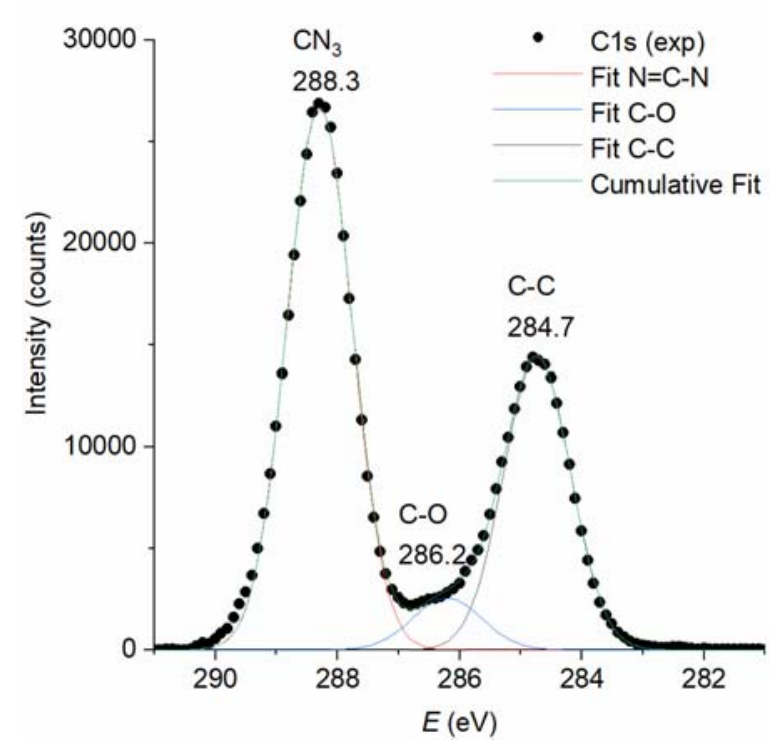

Figure S2. C 1s XPS of mpg-CN. 


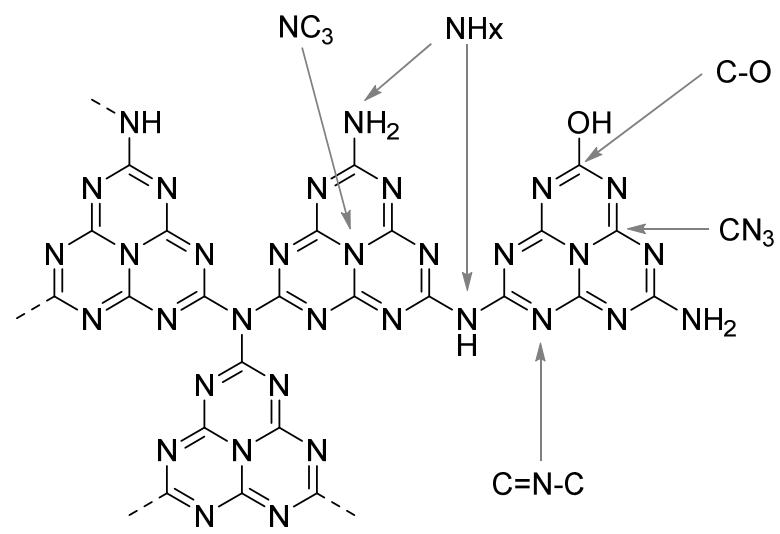

Figure S3. A fragment of mpg-CN structure based on characterization data.

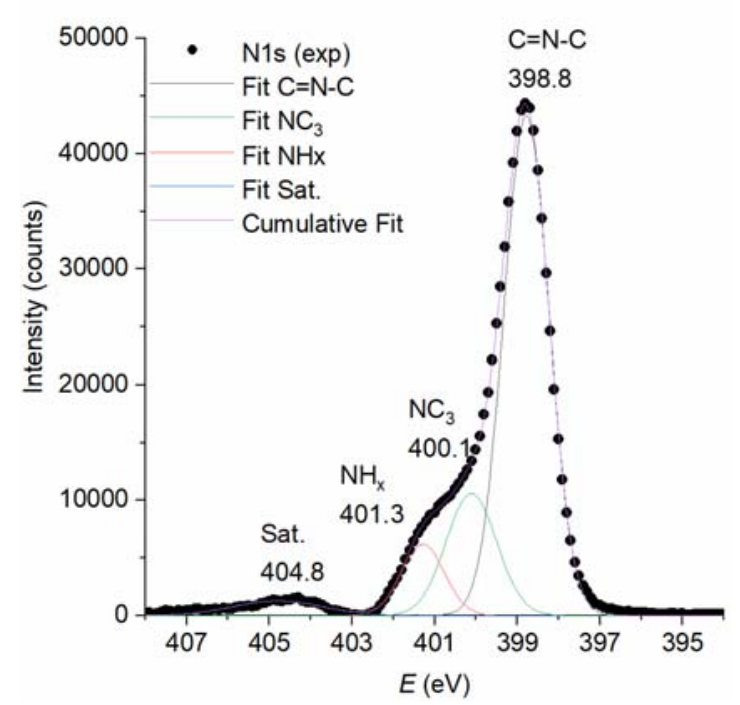

Figure S4. N 1s XPS of mpg-CN.

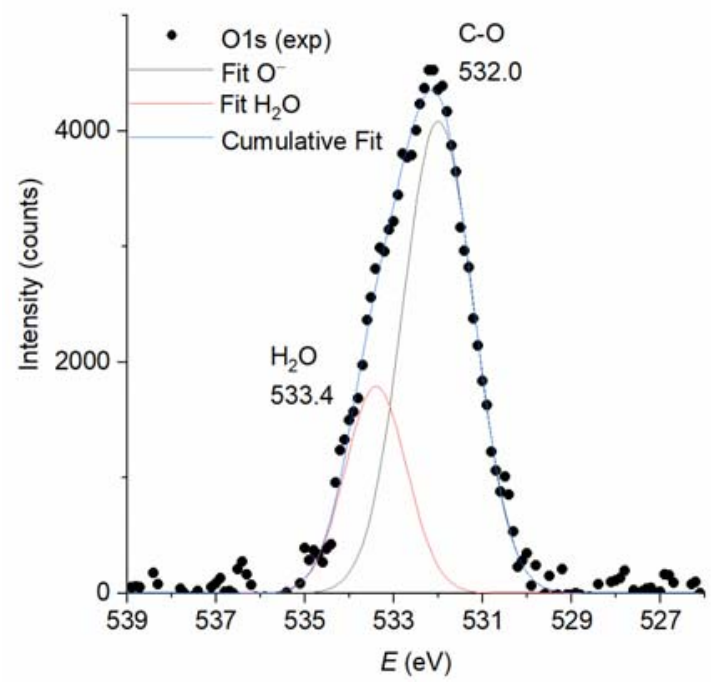

Figure S5. O 1s XPS of mpg-CN. 
Surface area of mpg-CN was determined from $\mathrm{N}_{2}$ absorption isotherm to be $186 \mathrm{~m}^{2} \mathrm{~g}^{-1}$ (Figure S6a). Mpg-CN features pores in the range from 5 to $44 \mathrm{~nm}$ and maximum at $13 \mathrm{~nm}$ (Figure S6b). As evidenced by PXRD, mpg-CN is amorphous material (Figure S7). The diffraction pattern features a peak at $\sim 27^{\circ}$ that is related to interplanar periodicity in the material with the distance between layers of $\sim 0.326 \mathrm{~nm} \cdot{ }^{10,11}$ Less pronounced peak at $\sim 13^{\circ}$ is assigned to intraplanar periodicity in the material with the corresponding distance of $\sim 0.68 \mathrm{~nm}$.
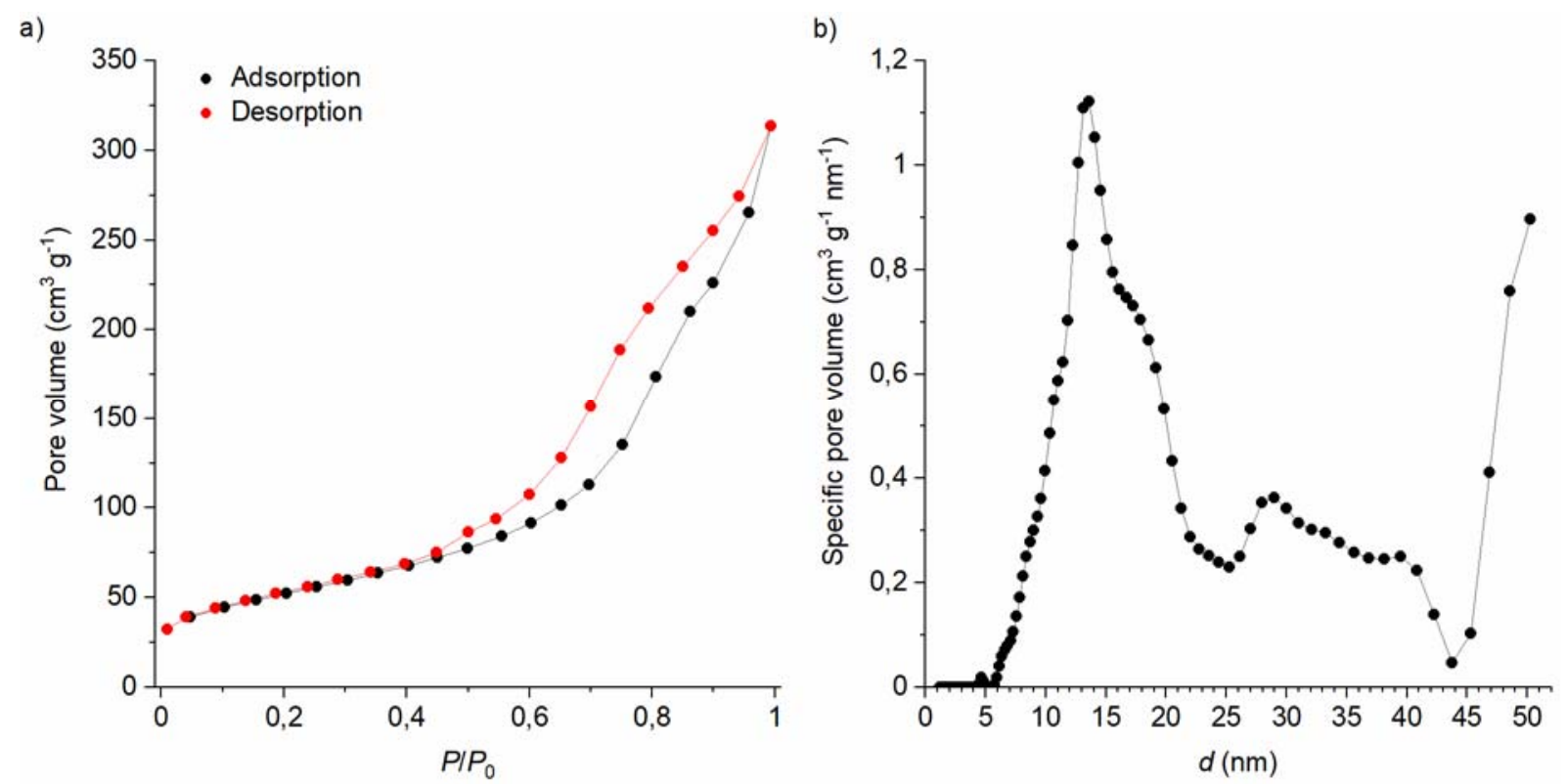

Figure S6. (a) $\mathrm{N}_{2}$ sorption isotherm recorded at $77 \mathrm{~K}$. (b) Pore size distribution in mpg-CN.

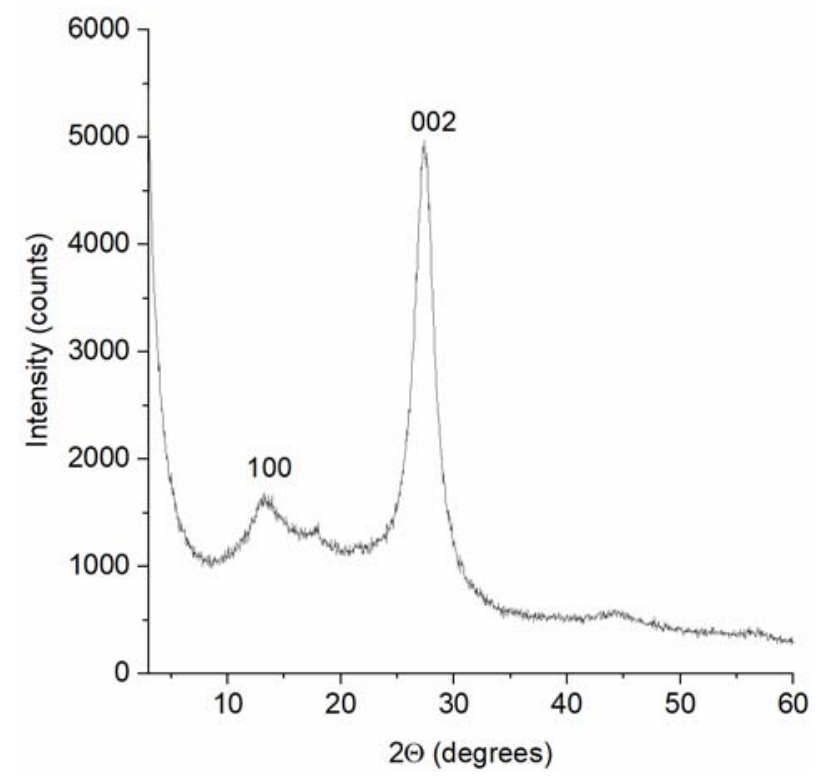

Figure S7. PXRD pattern of mpg-CN. 
DRUV-vis spectrum of mpg-CN features steep onset of absorption at $\sim 460 \mathrm{~nm}$ that is related to electron transition between conduction and valence band, also known in literature as $\pi-\pi *$ transitions, (Figure S8a). ${ }^{11}$ The optical band gap calculated from Tauc plot assuming that mpg$\mathrm{CN}$ is a direct semiconductor is $2.7 \mathrm{eV}$ (Figure S8b). ${ }^{11}$ A band at $\sim 650 \mathrm{~nm}$ of lower intensity is assigned to $n-\pi^{*}$ transitions that involve $\mathrm{N}$ - and O-electron pairs (Figure S8a). ${ }^{12,13}$ mpg-CN features broad fluorescence signal with maximum at $508 \mathrm{~nm}$ related radiative recombination of photogenerated electrons and holes (Figure S9).

a)

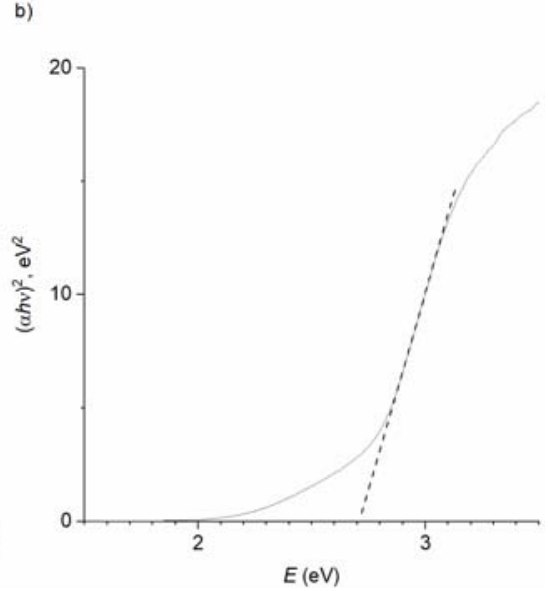

Figure S8. (a) DRUV-vis absorption spectrum. (b) Tauc plot of mpg-CN.

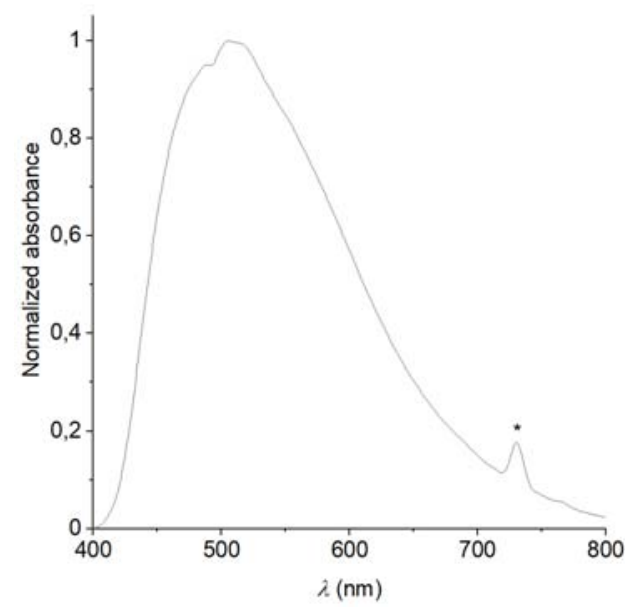

Figure S9. Steady state PL spectrum of mpg-CN. The sample was excited with $\lambda=365 \mathrm{~nm}$. Asterisk denotes $2^{\text {nd }}$ order excitation light diffraction.

Morphology of mpg-CN is represented by agglomerated particles with the dimeter ranging from few hundred nanometers to few micrometers (Figure S10). TEM image revealed mesoporous structure of the material with pores ranging from $<10 \mathrm{~nm}$ up to $\sim 25 \mathrm{~nm}$ (Figure S11a) that is consistent with the results of pore-size distribution analysis derived from $\mathrm{N}_{2}$ sorption (Figure S6b). HR-TEM image and the results of the corresponding FFT analysis (Figure S11b) 
confirmed PXRD data that mpg- $\mathrm{CN}$ is amorphous material, albeit possessing layered structure with the distance between layers of $\sim 0.352 \mathrm{~nm}$ as seen from the profile analysis (Figure S11c).

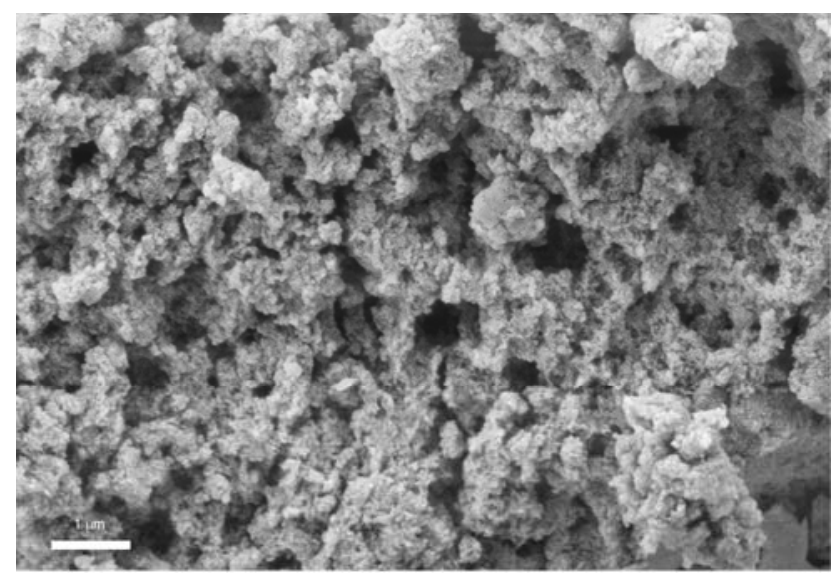

Figure S10. SEM image of mpg-CN.
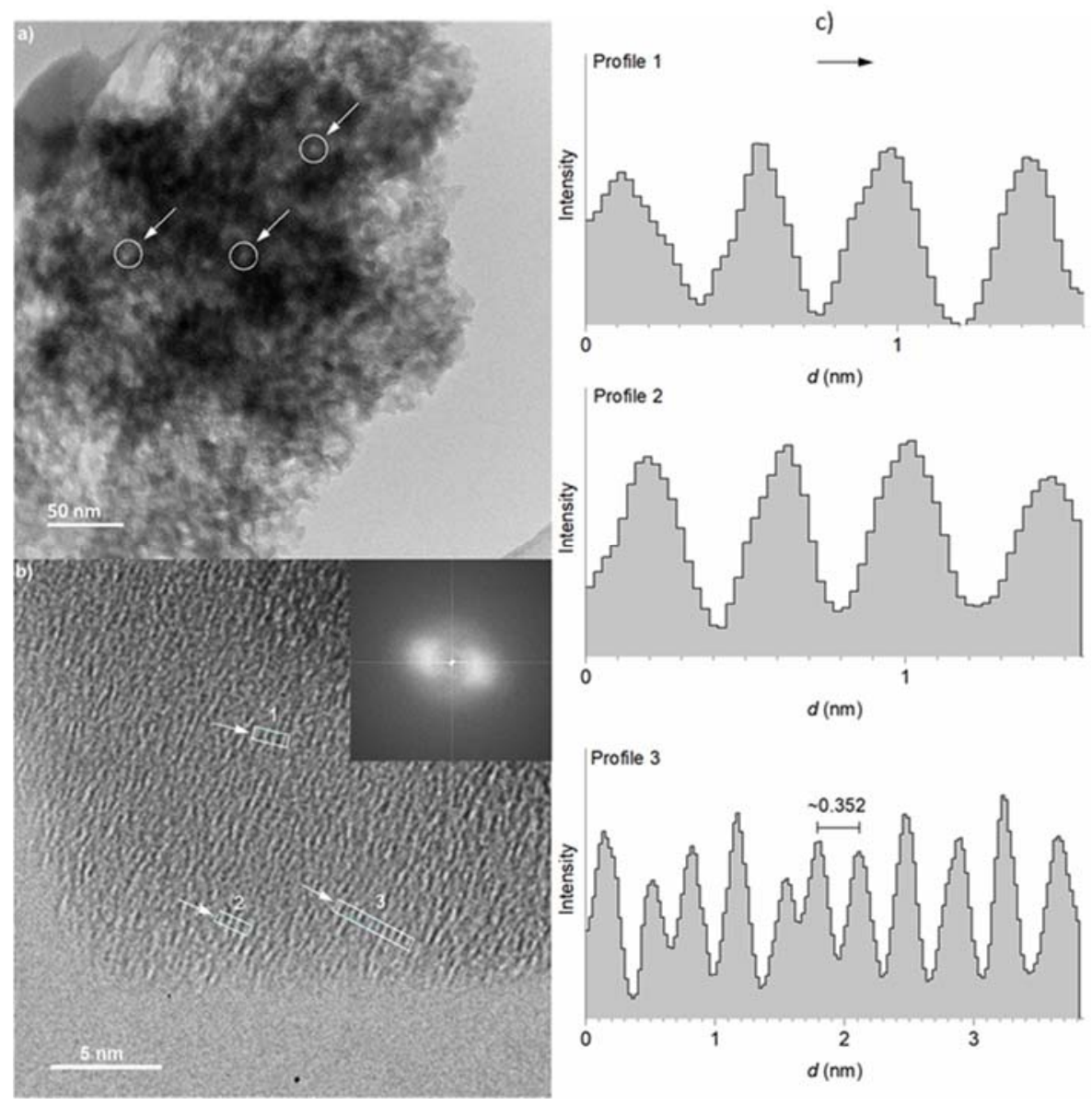

Figure S11. (a) Overview TEM image of mpg-CN image, Circles mark pores in the structure of the material. (b) HR-TEM image of mpg-CN with Fast Fourier Transform (FFT) in inset, arrows denote directions of view in profile analysis. (c) Profiles of the corresponding areas of HR-TEM image. 


\section{Description of general synthetic procedures}

\subsection{Synthesis of mpg-CN photocatalyst}

mpg-CN has been synthesized according to the reported literature procedure. ${ }^{1}$

\subsection{General procedure 1 (GP1) for Aryl Bromides}

A $5 \mathrm{~mL}$ crimp vial equipped with a magnetic stirring bar was charged with, bromo arene $(0.2$ mmol, 1.0 equiv ), mpg-CN (10.0 mg), NiBr2•glyme (0.01 mmol, $5 \mathrm{~mol} \%$.) and 2,2'-bipyridine (0.01 mmol, $5 \mathrm{~mol} \%)$. Subsequently, $1.0 \mathrm{~mL}$ of $N, N$-dimethylacetamide (DMA) was added followed by addition of 2,6 -lutidine $(0.6 \mathrm{mmol}, 3.0$ equiv $)$. The vial was sealed, and the reaction mixture was then introduced to a nitrogen atmosphere via "freeze-pump-thaw" cycles $(\times 3)$ with a syringe needle, keeping the vacuum approx. 5 mbar. After that the reaction mixture was irradiated with $5 \mathrm{~W} 455( \pm 15) \mathrm{nm}$ LEDs through the plane bottom side of the crimp vial (see Figure S12 for the reaction set up) and stirred intensely. The temperature was maintained at 30 ${ }^{\circ} \mathrm{C}$ by cooling with built-in cooling fan. The reaction progress was monitored by GC or TLC analysis. After completion (approx. $48 \mathrm{~h}$ ), the reaction mixture was transferred to a centrifuge tube (approx. $3 \times 3 \mathrm{~mL}$ of ethyl acetate was used in order to transfer the reaction mixture completely), and the mixture was centrifuged at $4000 \mathrm{rpm}$ (time approx. 1-10 minutes) until the heterogeneous photocatalyst mpg-CN precipitates. The supernatant liquid was collected in a separating funnel, and the solid in the centrifuge tube was washed three times using approx. $10 \mathrm{~mL}$ of ethyl acetate and added to the same separating funnel. Then, the mixture was washed with approx. $10 \mathrm{~mL}$ of distilled water and $5 \mathrm{~mL}$ of brine solution the organic layer was collected. The water layer was extracted again with ethyl acetate $(2 \times 10 \mathrm{~mL})$. The combined organic layers were washed with $5 \mathrm{~mL}$ of $0.1 \mathrm{~N} \mathrm{HCl}$ solution and the organic layer was collected. The combined organic layers were dried over $\mathrm{MgSO}_{4}$, filtered and concentrated in vacuum. Purification of the crude product was achieved by flash column chromatography.

\subsection{General procedure 2 (GP2) for Aryl Chlorides}

A $5 \mathrm{~mL}$ crimp vial equipped with a magnetic stirring bar was charged with, chloro arene $(0.2$ mmol, 1.0 equiv ), mpg-CN (10.0 mg), $\mathrm{NiCl}_{2} \bullet$ glyme (0.01 mmol, $5 \mathrm{~mol} \%$.) and 2,2'-bipyridine (0.01 mmol, $5 \mathrm{~mol} \%$ ). Subsequently, $1.0 \mathrm{~mL}$ of $N, N$-dimethylacetamide (DMA) was added followed by addition of 2,6-lutidine $(0.6 \mathrm{mmol}, 3.0$ equiv $)$. The vial was sealed, and the reaction mixture was then introduced to a nitrogen atmosphere via "freeze-pump-thaw" cycles $(\times 3)$ with a syringe needle, keeping the vacuum approx. 5 mbar. After that the reaction mixture was 
irradiated with $5 \mathrm{~W} 455( \pm 15)$ nm LEDs through the plane bottom side of the crimp vial (Figure S12) and stirred intensely. The temperature was maintained at $30{ }^{\circ} \mathrm{C}$ by cooling with built-in cooling fan. The reaction progress was monitored by GC or TLC analysis. After completion (approx. 72h), the reaction mixture was transferred to a centrifuge tube (approx. $3 \times 3 \mathrm{~mL}$ of ethyl acetate was used in order to transfer the reaction mixture completely), and the mixture was centrifuged at $4000 \mathrm{rpm}$ (time approx. 1-10 minutes) until the heterogeneous photocatalyst mpg-CN precipitates. The supernatant liquid was collected in a separating funnel, and the solid in the centrifuge tube was washed three times using approx. $10 \mathrm{~mL}$ of ethyl acetate and added to the same separating funnel. Then, the mixture was washed with approx. $10 \mathrm{~mL}$ of distilled water and $5 \mathrm{~mL}$ of brine solution the organic layer was collected. The water layer was extracted again with ethyl acetate $(2 \times 10 \mathrm{~mL})$. The combined organic layers were washed with $5 \mathrm{~mL}$ of $0.1 \mathrm{~N} \mathrm{HCl}$ solution and the organic layer was collected. The combined organic layers were dried over $\mathrm{MgSO}_{4}$, filtered and concentrated in vacuum. Purification of the crude product was achieved by flash column chromatography.
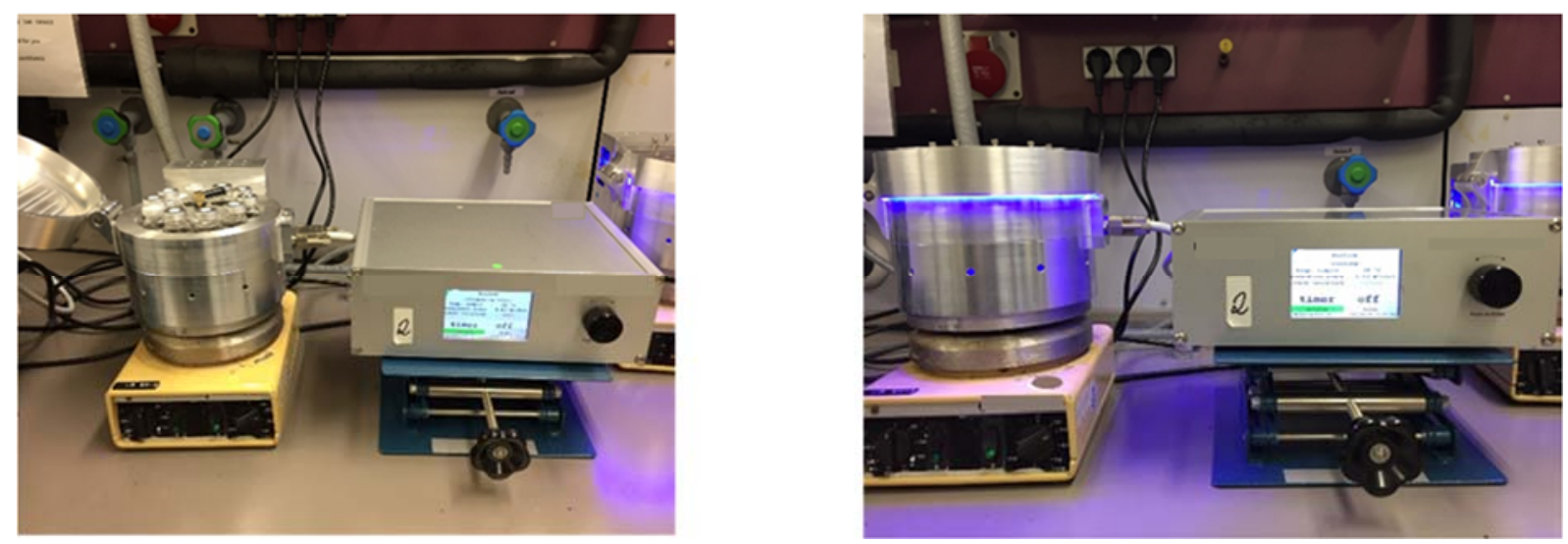

Figure S12. Photograph for the photochemical reaction set-up.

\subsection{General procedure 3 (GP3) for Heteroaryl Halides}

A $5 \mathrm{~mL}$ crimp vial equipped with a magnetic stirring bar was charged with, halo (het)arene $(0.2$ mmol, 1.0 equiv ), mpg-CN (10.0 mg), $\mathrm{NiX}_{2} \bullet$ glyme $(0.01 \mathrm{mmol}, 5 \mathrm{~mol} \%$.) (in case of bromoarene $\mathrm{NiBr}_{2} \bullet$ glyme and in case of chloro (het)arene $\mathrm{NiCl}_{2} \bullet$ glyme) and 2,2'-bipyridine (0.01 mmol, $5 \mathrm{~mol} \%$ ). Subsequently, $1.0 \mathrm{~mL}$ of $N, N$-dimethylacetamide (DMA) was added followed by addition of 2,6-lutidine $(0.6 \mathrm{mmol}, 3.0$ equiv $)$. The vial was sealed, and the reaction mixture was then introduced to a nitrogen atmosphere via "freeze-pump-thaw" cycles $(\times 3)$ with a syringe needle, keeping the vacuum approx. 5 mbar. After that the reaction mixture was irradiated with $5 \mathrm{~W} 455( \pm 15) \mathrm{nm}$ LEDs through the plane bottom side of the crimp vial (Figure S12) and stirred intensely. The temperature was maintained at $30{ }^{\circ} \mathrm{C}$ by cooling with built-in 
cooling fan. The reaction progress was monitored by GC or TLC analysis. After completion (approx. 48-72h), the reaction mixture was transferred to a centrifuge tube (approx. $3 \times 3 \mathrm{~mL}$ of ethyl acetate was used in order to transfer the reaction mixture completely), and the mixture was centrifuged at $4000 \mathrm{rpm}$ (time approx. 1-10 minutes) until the heterogeneous photocatalyst mpg-CN precipitates. The supernatant liquid was collected in a round bottom flask, and the solid in the centrifuge tube was washed three times using approx. $10 \mathrm{~mL}$ of ethyl acetate and added to the same round bottom flask. The combined organic layers were directly concentrated under reduced pressure. Purification of the crude product was achieved by flash column chromatography.

\subsection{General Procedure 4 (GP4) for $\mathrm{C}\left(\mathrm{sp}^{3}\right)-\mathrm{H}$ arylation of amides}

A $5 \mathrm{~mL}$ crimp vial equipped with a magnetic stirring bar was charged with, chloro (het)arene (0.2 mmol, 1.0 equiv ), mpg-CN (10.0 mg), NiCl $\bullet$ glyme (0.01 mmol, $5 \mathrm{~mol} \%$.) and 2,2'bipyridine $(0.01 \mathrm{mmol}, 5 \mathrm{~mol} \%)$. Subsequently, $1.0 \mathrm{~mL}$ of C-H precursors (for those which can be used as solvents, otherwise, $2.0 \mathrm{mmol}, 10$ equiv. of $\mathrm{C}-\mathrm{H}$ precursors in combination with 0.8 $\mathrm{mL}$ of $\mathrm{CH}_{3} \mathrm{CN}$ as solvent and $10 \mathrm{~mol} \%$ tetrabutylammonium chloride as an additive) was added followed by addition of 2,6-lutidine ( $0.6 \mathrm{mmol}, 3.0$ equiv $)$. The vial was sealed, and the reaction mixture was then introduced to a nitrogen atmosphere via "freeze-pump-thaw" cycles $(\times 3)$ with a syringe needle, keeping the vacuum approx. 5 mbar. After that the reaction mixture was irradiated with $5 \mathrm{~W} 455( \pm 15) \mathrm{nm}$ LEDs through the plane bottom side of the crimp vial (Figure S12) and stirred intensely. The temperature was maintained at $30^{\circ} \mathrm{C}$ by cooling with built-in cooling fan. The reaction progress was monitored by GC or TLC analysis. After completion (approx. 72h), the reaction mixture was transferred to a centrifuge tube (approx. $3 \times 3 \mathrm{~mL}$ of ethyl acetate was used in order to transfer the reaction mixture completely), and the mixture was centrifuged at $4000 \mathrm{rpm}$ (time approx. 1-10 minutes) until the heterogeneous photocatalyst mpg-CN precipitates. The supernatant liquid was collected in a separating funnel, and the solid in the centrifuge tube was washed three times using approx. $10 \mathrm{~mL}$ of ethyl acetate and added to the same separating funnel. Then, the mixture was washed with approx. $10 \mathrm{~mL}$ of distilled water and $5 \mathrm{~mL}$ of brine solution the organic layer was collected. The water layer was extracted again with ethyl acetate $(2 \times 10 \mathrm{~mL})$. The combined organic layers were washed with $5 \mathrm{~mL}$ of $0.1 \mathrm{~N} \mathrm{HCl}$ solution and the organic layer was collected. The combined organic layers were dried over $\mathrm{MgSO}_{4}$, filtered and concentrated in vacuum. Purification of the crude product was achieved by flash column chromatography. 


\subsection{General Procedure 5 (GP5) for gram scale synthesis}

A custom-made glass-photoreactor ("Tauchschachtreaktor") was charged with a magnetic stirring bar, the bromoarene (6.0 mmol, 1.0 equiv.), mpg-CN (150.0 mg), and $\mathrm{NiBr}_{2} \bullet$ glyme (0.3 mmol, $5 \mathrm{~mol} \%$.) and 2,2'-bipyridine ( $0.3 \mathrm{mmol}, 5 \mathrm{~mol} \%)$. Subsequently, $60 \mathrm{~mL}$ of $N, N$ dimethylacetamide (DMA) (c = $0.1 \mathrm{M})$ was added followed by addition of 2,6-lutidine (12 mmol, 2.0 equiv). The reaction mixture was placed in a nitrogen atmosphere via "freeze-pumpthaw" cycles $(\times 3)$. The reaction mixture was then irradiated at $25^{\circ} \mathrm{C}$ under nitrogen atmosphere using blue LED arrays (OSRAM Oslon SSL 80 LT-2010, $\lambda=451 \mathrm{~nm}, 700 \mathrm{~mA}$ ) generating a total radiant flux of $12 \mathrm{~W}$ (Figure S13) and stirred intensely. After completion (approx. 60h), the reaction mixture was transferred to a centrifuge tube (approx. 5 X $15 \mathrm{~mL}$ of ethyl acetate was used in order to transfer the reaction mixture completely), and the mixture was centrifuged until the mpg-CN precipitates. The supernatant liquid was then collected in a separating funnel, and the solid in the centrifuge tube was washed 5 times using approx. $15 \mathrm{~mL}$ ethyl acetate and the solvent added to the same separating funnel. Then, approx. $60 \mathrm{~mL}$ of brine solution was added, shaken, and the organic layer was collected. The water layer was extracted with ethyl acetate $(3 \times 15 \mathrm{~mL})$. The combined organic layers were washed with $60 \mathrm{~mL}$ of $0.1 \mathrm{~N} \mathrm{HCl}$ solution, the organic layer was collected and dried over $\mathrm{MgSO}_{4}$, filtered and concentrated in vacuum. Purification of the crude product by column chromatography.

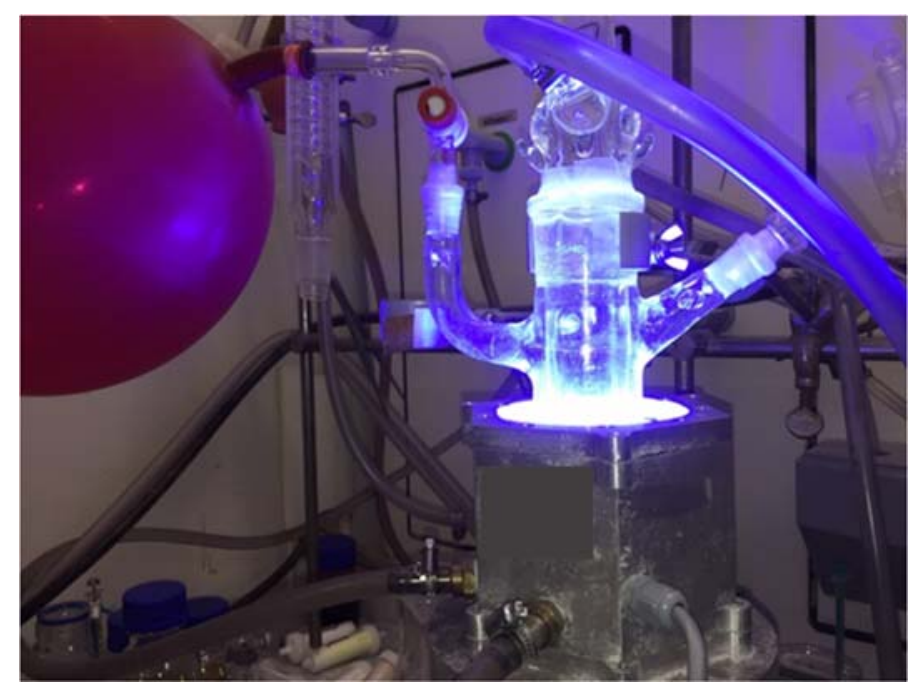

Figure S13. Photograph for the large-scale photochemical reaction set-up ("Tauchschacht Photoreactor''). 


\section{Optimization Studies}

Table S3. Screening of Bases.

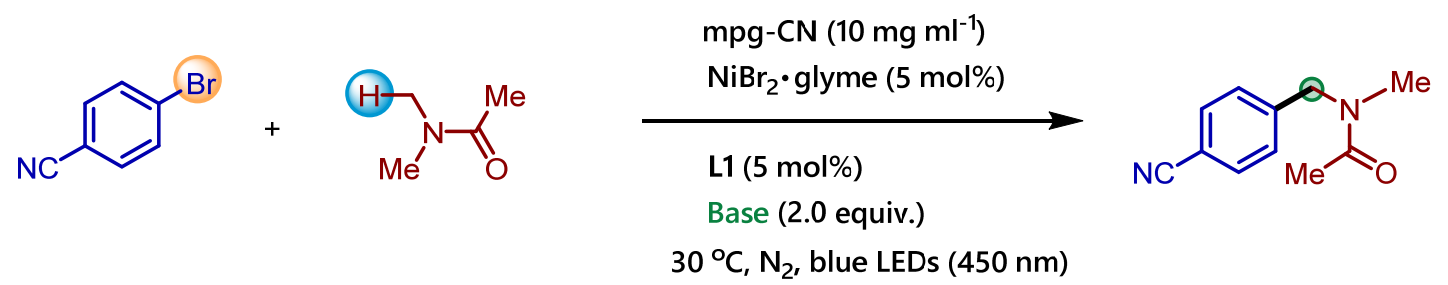

\begin{tabular}{ccc}
\hline Entry $^{a}$ & Base & Yield $^{b}$ \\
\hline 1. & $\mathrm{~K}_{2} \mathrm{CO}_{3}$ & $11 \%$ \\
2. & $\mathrm{~K}_{3} \mathrm{PO}_{4}$ & $13 \%$ \\
3. & $\mathrm{~K}_{2} \mathrm{HPO}_{4}$ & $16 \%$ \\
4. & $\mathrm{DABCO}$ & $12 \%$ \\
5. & $\mathrm{DBU}$ & $15 \%$ \\
6. & $\mathrm{DBN}$ & $<5 \%$ \\
7. & 2,6 -Lutidine & $52 \%$ \\
8. & $2,4,6-$ Collindine & $34 \%$ \\
9. & Pyridine & $28 \%$ \\
10. & DMAP & $18 \%$ \\
\hline
\end{tabular}

${ }^{a}$ Reaction Condition: 4-bromobenzonitrile (0.2 mmol, $\left.36.4 \mathrm{mg}\right)$, mpg-CN (10 mg), NiBr $2 \cdot$ glyme (5 mol\%, $\left.3.2 \mathrm{mg}\right)$, L1 $(5 \mathrm{~mol} \%, 2.7 \mathrm{mg})$, Base (2.0 equiv.), DMA ( $1 \mathrm{~mL})$ under nitrogen atmosphere and blue light irradiation for 48 h. ${ }^{b}$ Yields were determined by GC-FID using 1,4-dimethoxybenzene as internal standard.

Table S4. Screening of Ligands.

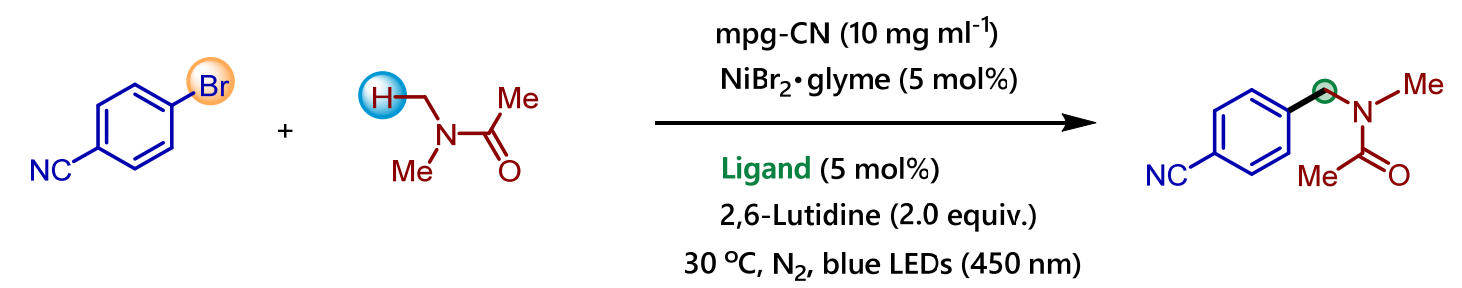

\begin{tabular}{ccc}
\hline Entry $^{a}$ & Ligand & Yield $^{b}$ \\
\hline 1. & L1 & $52 \%$ \\
2. & L2 & $43 \%$ \\
3. & L3 & $54 \%$ \\
4. & L4 & $28 \%$ \\
5. & L5 & $65 \%$ \\
6. & $\mathbf{L 6}$ & $74 \%$ \\
7. & $\mathbf{L 7}$ & $22 \%$ \\
8. & $\mathbf{L 8}$ & $72 \%$ \\
9. & $\mathbf{L 9}$ & $13 \%$ \\
\hline
\end{tabular}

${ }^{a}$ Reaction Condition: 4-bromobenzonitrile (0.2 mmol, $\left.36.4 \mathrm{mg}\right)$, mpg-CN (10 mg), $\mathrm{NiBr}_{2} \cdot$ glyme (5 mol\%, $\left.3.2 \mathrm{mg}\right)$, Ligand $(5 \mathrm{~mol} \%)$, 2,6-lutidine ( $48 \mu \mathrm{L}, 2.0$ equiv.), DMA $(1 \mathrm{~mL})$ under nitrogen atmosphere and blue light irradiation for $48 \mathrm{~h}$. ${ }^{\text {}}$ Yields were determined by GC-FID using 1,4-dimethoxybenzene as internal standard. 


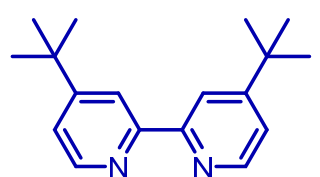

L1

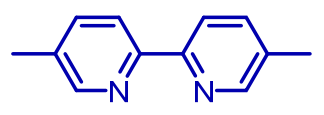

L5<smiles>C1COC(C2=NCCO2)=N1</smiles>

L9

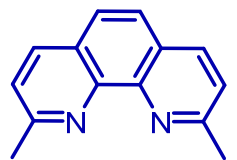

L2

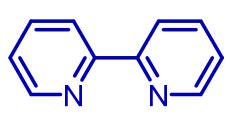

L6

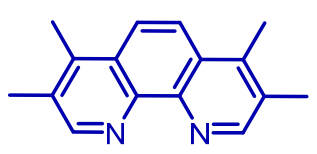

L3

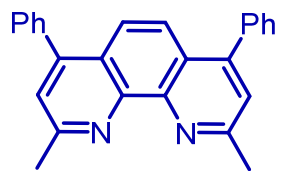

L4<smiles>Cc1cccc(-c2cccc(C)n2)n1</smiles>

L7

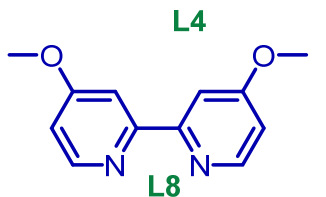

Table S5. Screening of Additives and other variables.
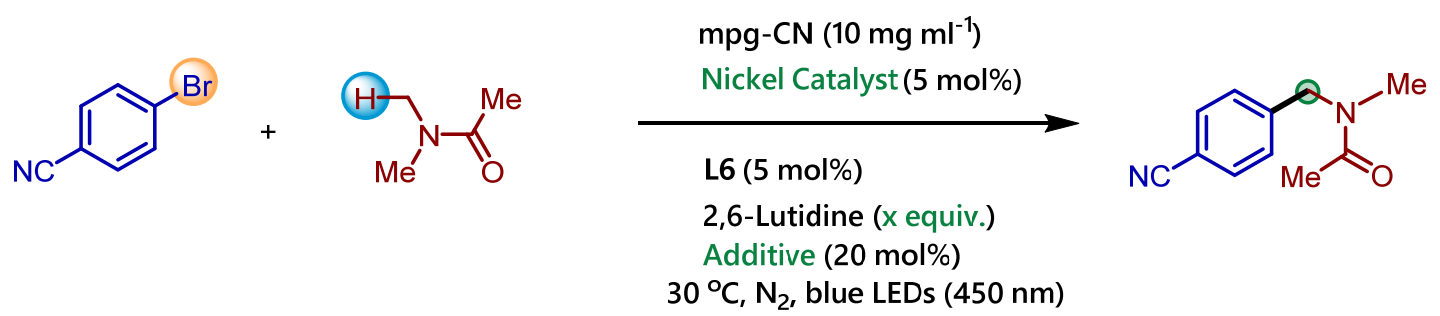

\begin{tabular}{|c|c|c|c|c|}
\hline Entry $^{a}$ & Nickel source & Base & Additive & Yield $^{b}$ \\
\hline 1. & $\mathrm{NiBr}_{2} \cdot$ glyme & 2,6-Lutidine & $\mathrm{NaBr}$ & $48 \%$ \\
\hline 2. & $\mathrm{NiBr} 2 \cdot$ glyme & 2,6-Lutidine & $\mathrm{CBr}_{4}$ & $<5 \%$ \\
\hline 3. & $\mathrm{NiBr} 2 \cdot$ glyme & 2,6-Lutidine & $\mathrm{TBAB}^{c}$ & $7 \%$ \\
\hline 4. & $\mathrm{NiBr} 2 \cdot$ glyme & $\begin{array}{c}\text { 2,6-Lutidine } \\
\text { (1.2 equiv.) }\end{array}$ & - & $62 \%$ \\
\hline 5. & $\mathrm{NiBr}_{2} \cdot$ glyme & $\begin{array}{l}\text { 2,6-Lutidine } \\
\text { (3.0 equiv.) }\end{array}$ & - & $90 \%(85 \%)^{d}$ \\
\hline 6. & $\mathrm{NiBr}_{2}$ & $\begin{array}{c}\text { 2,6-Lutidine } \\
\text { (3.0 equiv.) }\end{array}$ & - & $18 \%$ \\
\hline 7. & $\mathrm{NiBr}_{2} \cdot 3 \mathrm{H}_{2} \mathrm{O}$ & $\begin{array}{l}\text { 2,6-Lutidine } \\
\text { (3.0 equiv.) }\end{array}$ & - & $72 \%$ \\
\hline 8. & $\mathrm{Ni}(\mathrm{bpy})_{3} \mathrm{Br}_{2}$ & $\begin{array}{c}\text { 2,6-Lutidine } \\
\text { (3.0 equiv.) }\end{array}$ & - & $46 \%$ \\
\hline
\end{tabular}


Table S6. Control Experiments.

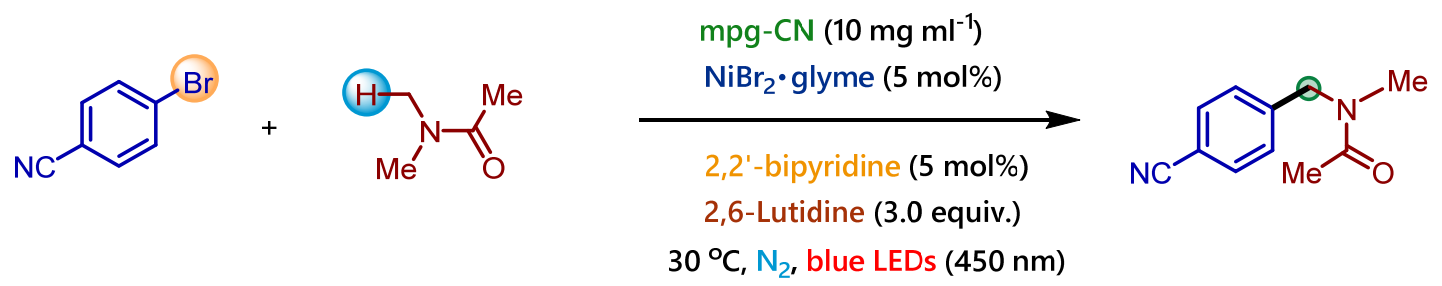

\begin{tabular}{ccc}
\hline Entry $^{a}$ & Conditions & Yield $^{b}$ \\
\hline 1. & Without mpg-CN & ND \\
2. & Without NiBr2'glyme & ND \\
3. & Without 2,2'-bipyridine & $<5 \%$ \\
4. & Without 2,6-Lutidine & $11 \%$ \\
5. & Without Light & \\
6. & Without degassing \& $\mathrm{N}_{2}$ & $74 \%$ \\
7. & Under air & $60 \%$ \\
\hline
\end{tabular}

${ }^{a}$ Reaction Condition: 4-bromobenzonitrile $(0.2 \mathrm{mmol}, 36.4 \mathrm{mg})$, mpg-CN (10 mg), Nickel catalyst (5 mol\%), 2,2'bipyridine $(5 \mathrm{~mol} \%, 1.6 \mathrm{mg})$, 2,6-lutidine, DMA $(1 \mathrm{~mL})$ under nitrogen atmosphere and blue light irradiation for $48 \mathrm{~h} .{ }^{b}$ Yields were determined by GC-FID using 1,4-dimethoxybenzene as internal standard. ND, Not detected.

Unfortunately, when the reaction was performed with $\mathrm{N}$-Boc pyrrolidine as $\mathrm{C}-\mathrm{H}$ precursor instead of DMA under the standard reaction condition, it failed to afford the desired product. 


\section{Scope and analytical data}

\section{$N$-(4-cyanobenzyl)- $N$-methylacetamide (1)}<smiles>CC(=O)N(C)Cc1ccc(C#N)cc1</smiles>

The compound was prepared according to the General Procedure 1 using 4-bromobenzonitrile (36.4 mg, $0.2 \mathrm{mmol}$ ), mpg-CN (10.0 mg), NiBr ${ }^{\bullet}$ glyme (3.2 mg, 5 mol\%), 2,2'-bipyridine (1.6 mg 5 mol\%), $N, N$-dimethylacetamide $(1.0 \mathrm{~mL})$ and 2,6-lutidine $(70 \mu \mathrm{L} 0.6 \mathrm{mmol})$. The reaction mixture was irradiated under nitrogen atmosphere for 48h using blue LED $(455( \pm 15) \mathrm{nm})$. The reaction mixture was then subjected to the work-up procedure as described in the General Procedure 1. Purification was performed using flash chromatography on silica gel using $\mathrm{PE} /$ EtOAc solvent mixture as eluent to provide the title compound in $85 \%$ yield $(31.9 \mathrm{mg})$. Following the General Procedure 2 the same compound was synthesized from 4chlorobenzonitrile (27.4 mg, $0.2 \mathrm{mmol})$, mpg-CN (10.0 mg), NiCl${ }_{2}^{\bullet}$ glyme (2.2 mg, $5 \mathrm{~mol} \%$ ), 2,2'-bipyridine (1.6 mg $5 \mathrm{~mol} \%), N, N$-dimethylacetamide $(1.0 \mathrm{~mL})$ and 2,6-lutidine (70 $\mu \mathrm{L} 0.6$ mmol) with an isolated yield of $89 \%$ (33.4 mg). Following the General Procedure 5 the same compound was synthesized from 4-bromobenzonitrile $(1.1 \mathrm{~g}, 6.0 \mathrm{mmol})$ with an isolated yield of $83 \%(936 \mathrm{mg}) .{ }^{1} \mathrm{H} \mathrm{NMR}\left(300 \mathrm{MHz}, \mathrm{CDCl}_{3}\right) \delta 7.67(\mathrm{~d}, J=8.2 \mathrm{~Hz}) \& 7.61(\mathrm{~d}, J=8.2 \mathrm{~Hz}, 2 \mathrm{H}$ combined), $7.34(\mathrm{~d}, J=8.1 \mathrm{~Hz}) \& 7.29(\mathrm{~d}, J=8.1 \mathrm{~Hz}, 2 \mathrm{H}$ combined), $4.62(\mathrm{~s}) \& 4.59(\mathrm{~s}, 2 \mathrm{H}$ combined), 2.96 (s) \& 2.94 (s, 3H combined), 2.17 (s) \& 2.12 (s, 3H combined). ${ }^{13} \mathrm{C}$ NMR (75 $\left.\mathrm{MHz}, \mathrm{CDCl}_{3}\right) \delta 171.0 \& 170.9,142.9 \& 142.1,132.8 \& 132.4,128.4 \& 126.9,118.7 \& 118.4$, $111.3 \& 111.2,53.9 \& 50.6,35.9 \& 33.9,21.7 \& 21.4$. HRMS (APCI): $\mathrm{m} / \mathrm{z}$ calculated. for $\mathrm{C}_{11} \mathrm{H}_{13} \mathrm{~N}_{2} \mathrm{O}[\mathrm{M}+\mathrm{H}]^{+}:$189.1022; found 189.1024.

\section{Methyl 4-(( $N$-methylacetamido)methyl)benzoate (2)}<smiles>CC(=O)c1ccc(CN(C)C(C)=O)cc1</smiles>

The compound was prepared according to the General Procedure 1 using ethyl 4-bromobenzoate (45.8 $\mathrm{mg}, 0.2 \mathrm{mmol}$ ), mpg-CN (10.0 mg), NiBr2•glyme (3.2 mg, 5 mol\%), 2,2'-bipyridine (1.6 mg $5 \mathrm{~mol} \%), N, N$-dimethylacetamide $(1.0 \mathrm{~mL})$ and 2,6-lutidine $(70 \mu \mathrm{L} 0.6 \mathrm{mmol})$. The reaction mixture was irradiated under nitrogen atmosphere for 48h using blue LED $(455( \pm 15)$ $\mathrm{nm})$. The reaction mixture was then subjected to the work-up procedure as described in the General Procedure 1. Purification was performed using flash chromatography on silica gel using PE/EtOAc solvent mixture as eluent to provide the title compound in $73 \%$ yield $(32.2$ mg). ${ }^{1} \mathrm{H}$ NMR (300 MHz, $\left.\mathrm{CDCl}_{3}\right) \delta 8.04-7.98$ (m) \& 7.98 - 7.92 (m, 2H combined), 7.29 $7.23(\mathrm{~m}) \& 7.24-7.18(\mathrm{~m}, 2 \mathrm{H}$ combined), $4.60(\mathrm{~s}) \& 4.55(\mathrm{~s}, 2 \mathrm{H}$ combined), $3.88(\mathrm{~s}), 3.86(\mathrm{~s}$, 
$3 \mathrm{H}$ combined), 2.91 (s) \& 2.90 (s, 3H combined), 2.14 (s) \& 2.10 (s, 3H combined). ${ }^{13} \mathrm{C}$ NMR $\left(75 \mathrm{MHz}, \mathrm{CDCl}_{3}\right) \delta 171.0 \& 170.9,166.8 \& 166.6,142.6 \& 141.8,130.3 \& 129.9,129.6$ $\& 129.2,127.82 \& 126.2,54.0 \& 50.4,52.2 \& 52.1,35.7 \& 33.9,21.8 \& 21.4$. HRMS (ESI): $m / z$ calculated. for $\mathrm{C}_{12} \mathrm{H}_{16} \mathrm{NO}_{3}[\mathrm{M}+\mathrm{H}]^{+}: 222.1125$; found 222.1128 .

\section{$N$-(4-acetylbenzyl)- $N$-methylacetamide (3)}<smiles>CC(=O)c1ccc(CN(C)C(C)=O)cc1</smiles>

The compound was prepared according to the General Procedure 1 using 1-(4-bromophenyl)ethan-1-one (39.8 mg, 0.2 mmol), mpg-CN (10.0 mg), NiBr2•glyme (3.2 mg, 5 mol\%), 2,2'bipyridine $(1.6 \mathrm{mg} 5 \mathrm{~mol} \%), N, N$-dimethylacetamide $(1.0 \mathrm{~mL})$ and 2,6-lutidine $(70 \mu \mathrm{L} 0.6$ $\mathrm{mmol})$. The reaction mixture was irradiated under nitrogen atmosphere for $48 \mathrm{~h}$ using blue LED $(455( \pm 15) \mathrm{nm})$. The reaction mixture was then subjected to the work-up procedure as described in the General Procedure 1. Purification was performed using flash chromatography on silica gel using PE/EtOAc solvent mixture as eluent to provide the title compound in 82\% yield (33.6 mg). ${ }^{1} \mathrm{H}$ NMR (300 MHz, $\left.\mathrm{CDCl}_{3}\right) \delta 7.95$ - 7.90 (m) \& 7.89 - 7.85 (m, 2H combined), 7.31 $7.26(\mathrm{~m}) \& 7.26-7.21(\mathrm{~m}, 2 \mathrm{H}$ combined), $4.60(\mathrm{~s}) \& 4.55$ (s, 2H combined), $2.91(\mathrm{~s}, 3 \mathrm{H}), 2.56$ (s) \& 2.55 (s, 3H combined), 2.14 (s) \& 2.10 (s, 3H combined). ${ }^{13} \mathrm{C} \mathrm{NMR}\left(75 \mathrm{MHz}, \mathrm{CDCl}_{3}\right) \delta$ $197.7 \& 197.5,171.0 \& 170.9,142.9 \& 142.0,136.6 \& 136.2,129.0 \& 128.7,128.0 \& 126.4$, $54.0 \& 50.4,35.8 \& 33.9,26.6,21.8 \& 21.4$. HRMS (APCI): $\mathrm{m} / \mathrm{z}$ calculated. for $\mathrm{C}_{12} \mathrm{H}_{16} \mathrm{NO}_{2}$ $[\mathrm{M}+\mathrm{H}]^{+}:$206.1176; found 206.1177.

\section{$N$-(4-formylbenzyl)- $N$-methylacetamide (4)}<smiles>CC(=O)N(C)Cc1ccc(C=O)cc1</smiles>

The compound was prepared according to the General Procedure 1 using 4-bromobenzaldehyde (37.0 mg, $0.2 \mathrm{mmol})$, mpg-CN (10.0 mg), NiBr $2 \bullet$ glyme (3.2 mg, 5 mol\%), 2,2'-bipyridine (1.6 mg $5 \mathrm{~mol} \%), N, N$-dimethylacetamide $(1.0 \mathrm{~mL})$ and 2,6-lutidine $(70 \mu \mathrm{L} 0.6 \mathrm{mmol})$. The reaction mixture was irradiated under nitrogen atmosphere for 48h using blue LED $(455( \pm 15)$ $\mathrm{nm})$. The reaction mixture was then subjected to the work-up procedure as described in the General Procedure 1. Purification was performed using flash chromatography on silica gel using PE/EtOAc solvent mixture as eluent to provide the title compound in $70 \%$ yield $(27.6$ mg). ${ }^{1} \mathrm{H}$ NMR (300 MHz, $\mathrm{CDCl}_{3}$ ) $\delta 9.99$ (s) \& 9.96 (s, $1 \mathrm{H}$ combined), 7.87 (d, J=8.2 Hz) \& $7.81(\mathrm{~d}, J=8.2 \mathrm{~Hz}, 2 \mathrm{H}$ combined), $7.37(\mathrm{~d}, J=8.1 \mathrm{~Hz}) \& 7.32(\mathrm{~d}, J=8.1 \mathrm{~Hz}, 2 \mathrm{H}), 4.63$ (s) \& 4.59 (s, 2H), $2.94(\mathrm{~s}) \& 2.94$ (s, 3H), 2.16 (s) \& 2.11 (s, 3H). ${ }^{13} \mathrm{C} \mathrm{NMR}\left(75 \mathrm{MHz}, \mathrm{CDCl}_{3}\right) \delta$ $191.9 \& 191.6,171.1 \& 171.0,144.5 \& 143.6,136.0 \& 135.6,130.4 \& 130.1,128.4 \& 126.8$, 
$54.1 \& 50.6,35.9 \& 34.0,21.8 \&$ 21.5. HRMS (ESI): $\mathrm{m} / \mathrm{z}$ calculated. for $\mathrm{C}_{12} \mathrm{H}_{14} \mathrm{NO}_{2}[\mathrm{M}+\mathrm{H}]^{+}$: 192.1019; found 192.1022.

\section{$N$-methyl-4-(( $N$-methylacetamido)methyl)benzamide (5)}<smiles>CNC(=O)c1ccc(CN(C)C(C)=O)cc1</smiles>

The compound was prepared according to the General Procedure 1 using 4-bromo- $N$-methylbenzamide (42.8 mg, 0.2 mmol), mpg-CN (10.0 mg), NiBr2•glyme (3.2 mg, 5 mol\%), 2,2'bipyridine (1.6 mg $5 \mathrm{~mol} \%), N, N$-dimethylacetamide $(1.0 \mathrm{~mL})$ and 2,6-lutidine (70 $\mu \mathrm{L} 0.6 \mathrm{mmol}$ ). The reaction mixture was irradiated under nitrogen atmosphere for $72 \mathrm{~h}$ using blue LED $(455( \pm 15) \mathrm{nm})$. The reaction mixture was then subjected to the workup procedure as described in the General Procedure 1. Purification was performed using flash chromatography on silica gel using $\mathrm{DCM} / \mathrm{MeOH}$ solvent mixture as eluent to provide the title compound in 74\% yield (32.6 mg). ${ }^{1} \mathrm{H}$ NMR (300 MHz, CDCl3) $\delta 7.84-7.74(\mathrm{~m}) \& 7.77-$ 7.67 (m, 2H combined), $7.29-7.23(\mathrm{~m}) \& 7.22-7.16(\mathrm{~m}, 2 \mathrm{H}$ combined), 6.49 (s, 1H), 4.60 (s) \& $4.55(\mathrm{~s}, 2 \mathrm{H}), 3.00(\mathrm{~d}, J=4.8 \mathrm{~Hz}) \& 2.99(\mathrm{~d}, J=4.8 \mathrm{~Hz}, 3 \mathrm{H}$ combined), $2.92(\mathrm{~s}, 3 \mathrm{H}), 2.16$ (s) \& $2.12(\mathrm{~s}, 3 \mathrm{H}) .{ }^{13} \mathrm{C} \mathrm{NMR}\left(101 \mathrm{MHz}, \mathrm{CDCl}_{3}\right) \delta 171.1 \& 171.0,168.0 \& 167.7,140.9 \&$ $140.1,134.2 \& 133.9,128.1 \& 127.7,127.3 \& 126.4,54.1 \& 50.53,35.83 \& 33.97,26.9,21.89$ \& 21.54. HRMS (ESI): $\mathrm{m} / \mathrm{z}$ calculated. for $\mathrm{C}_{12} \mathrm{H}_{17} \mathrm{~N}_{2} \mathrm{O}_{2}[\mathrm{M}+\mathrm{H}]^{+}:$221.1285; found 222.1286.

\section{$N$-(4-acetamidobenzyl)- $N$-methylacetamide (6)}

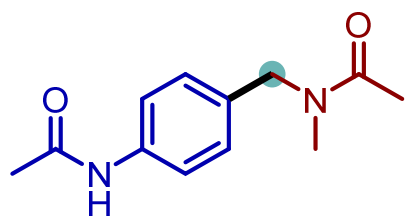

The compound was prepared according to the General Procedure 1 using $\mathrm{N}$-(4-bromophenyl)acetamide (42.8 mg, 0.2 mmol), mpg CN (10.0 mg), NiBr2•glyme (3.2 mg, 5 mol\%), 2,2'bipyridine (1.6 mg $5 \mathrm{~mol} \%), N, N$-dimethylacetamide $(1.0 \mathrm{~mL})$ and 2,6-lutidine ( $70 \mu \mathrm{L} 0.6 \mathrm{mmol})$. The reaction mixture was irradiated under nitrogen atmosphere for $72 \mathrm{~h}$ using blue LED $(455( \pm 15) \mathrm{nm})$. The reaction mixture was then subjected to the workup procedure as described in the General Procedure 1. Purification was performed using flash chromatography on silica gel using $\mathrm{DCM} / \mathrm{MeOH}$ solvent mixture as eluent to provide the title compound in $63 \%$ yield $(27.7 \mathrm{mg}) .{ }^{1} \mathrm{H}$ NMR $\left(400 \mathrm{MHz}, \mathrm{CDCl}_{3}\right) \delta 7.50(\mathrm{~d}, J=8.3 \mathrm{~Hz}) \& 7.44$ (d, $J=8.3 \mathrm{~Hz}, 2 \mathrm{H}$ combined), 7.19 (d, $J=8.3 \mathrm{~Hz}) \& 7.11$ (d, $J=8.3 \mathrm{~Hz}, 2 \mathrm{H}$ combined), 4.54 (s) \& 4.48 (s, 2H combined), 2.92 (s) \& 2.91 (s, 3H combined), 2.18 (s) \& 2.17 (s, 3H combined), $2.15(\mathrm{~s}, 3 \mathrm{H}) .{ }^{13} \mathrm{C} \mathrm{NMR}\left(75 \mathrm{MHz}, \mathrm{CDCl}_{3}\right) \delta 171.3 \& 171.0,169.0 \& 168.9,138.0 \&$ $137.6,132.8 \& 131.8,128.5 \& 126.9,120.4 \& 120.3,54.0 \& 50.3,35.7 \& 33.8,24.4,21.9 \&$ 21.61. HRMS (APCI): $m / z$ calculated. for $\mathrm{C}_{12} \mathrm{H}_{17} \mathrm{~N}_{2} \mathrm{O}_{2}[\mathrm{M}+\mathrm{H}]^{+}:$221.1285; found 222.1286. 
<smiles>CC(=O)N(C)Cc1ccc(S(C)(=O)=O)cc1</smiles>

The compound was prepared according to the General Procedure 1 using 1-bromo-4-(methylsulfonyl)benzene (47.0 mg, $0.2 \mathrm{mmol})$, mpg-CN (10.0 mg), NiBr $\bullet$ glyme (3.2 mg, 5 mol\%), 2,2'-bipyridine (1.6 mg $5 \mathrm{~mol} \%$ ), $N, N$-dimethylacetamide $(1.0 \mathrm{~mL})$ and 2,6-lutidine $(70 \mu \mathrm{L} 0.6 \mathrm{mmol})$. The reaction mixture was irradiated under nitrogen atmosphere for $48 \mathrm{~h}$ using as described in the General Procedure 1. Purification was performed using flash chromatography on silica gel using DCM/MeOH solvent mixture as eluent to provide the title compound in $78 \%$ yield $(37.6 \mathrm{mg}) .{ }^{1} \mathrm{H}$ NMR $\left(300 \mathrm{MHz}, \mathrm{CDCl}_{3}\right) \delta 7.92(\mathrm{~d}, J=8.3 \mathrm{~Hz}) \&, 7.86$ (d, $J=8.3 \mathrm{~Hz}, 2 \mathrm{H}$ combined), $7.40(\mathrm{~d}, J=8.3 \mathrm{~Hz}) \& 7.35$ (d, $J=8.3 \mathrm{~Hz}, 2 \mathrm{H}$ combined), 4.63 (s) \& $4.60(\mathrm{~s}, 2 \mathrm{H}$ combined), 3.03 (s) \& 3.01 (s, 3H combined), 2.95 (s) \& 2.92 (s, 3H combined), 2.15 (s) \& 2.10 (s, 3H combined). ${ }^{13} \mathrm{C} \mathrm{NMR}\left(75 \mathrm{MHz}, \mathrm{CDCl}_{3}\right) \delta 171.0 \& 170.9$, $144.0 \& 143.2,140.1 \& 139.6,128.6 \& 128.2,127.7 \& 127.2,53.9 \& 50.5,44.6 \& 44.5,36.0$ \& 33.9, $21.7 \&$ 21.4. HRMS (ESI): $m / z$ calculated. for $\mathrm{C}_{11} \mathrm{H}_{16} \mathrm{NO}_{3} \mathrm{~S}[\mathrm{M}+\mathrm{H}]^{+}: 242.0845$; found 242.0848 .

\section{$N$-methyl- $N$-(4-((trifluoromethyl)sulfonyl)benzyl)acetamide (8)}<smiles>CC(=O)N(C)Cc1ccc(S(=O)(=O)C(F)(F)F)cc1</smiles>

The compound was prepared according to the General Procedure 1 using 1-bromo-4-((trifluoromethyl)sulfonyl)benzene (57.8 mg, $0.2 \mathrm{mmol})$, mpg-CN (10.0 mg), NiBr2•glyme (3.2 mg, 5 mol\%), 2,2'-bipyridine (1.6 mg 5 mol\%), $N, N$-dimethylacetamide $(1.0 \mathrm{~mL})$ and 2,6-lutidine $(70 \mu \mathrm{L} 0.6 \mathrm{mmol})$. The reaction mixture was irradiated under nitrogen atmosphere for $48 \mathrm{~h}$ using blue LED $(455( \pm 15) \mathrm{nm})$. The reaction mixture was then subjected to the work-up procedure as described in the General Procedure 1. Purification was performed using flash chromatography on silica gel using $\mathrm{DCM} / \mathrm{MeOH}$ solvent mixture as eluent to provide the title compound in 64\% yield $(37.7 \mathrm{mg}) .{ }^{1} \mathrm{H} \mathrm{NMR}\left(400 \mathrm{MHz}, \mathrm{CDCl}_{3}\right) \delta 8.04(\mathrm{~d}, J=$ $8.3 \mathrm{~Hz}) \& 7.98(\mathrm{~d}, J=8.3 \mathrm{~Hz}, 2 \mathrm{H}$ combined), $7.52(\mathrm{~d}, J=8.4 \mathrm{~Hz}) \& 7.48(\mathrm{~d}, J=8.4 \mathrm{~Hz}, 2 \mathrm{H}$ combined), 4.70 (s) \& 4.67 (s, 2H combined), 3.01 (s) \& 2.97 (s, 3H combined), 2.19 (s) \& 2.12 (s, 3H combined). ${ }^{13} \mathrm{C}$ NMR $\left(101 \mathrm{MHz}, \mathrm{CDCl}_{3}\right) \delta 171.2 \& 171.0,147.5 \& 146.8,131.6 \&$ $131.2,130.2 \& 130.2,129.1 \& 127.67,119.1(\mathrm{q}, J=325 \mathrm{~Hz}) \& 119.0(\mathrm{q}, J=325 \mathrm{~Hz}), 54.0 \&$ 50.7, $36.3 \& 34.1,21.7 \& 21.4$. HRMS (APCI): $\mathrm{m} / \mathrm{z}$ calculated. for $\mathrm{C}_{11} \mathrm{H}_{13} \mathrm{~F}_{3} \mathrm{NO}_{3} \mathrm{~S}[\mathrm{M}+\mathrm{H}]^{+}$: 296.0563; found 293.0564. 


\section{$N$-methyl- $N$-(4-(trifluoromethyl)benzyl)acetamide (9)}<smiles>CC(=O)N(C)Cc1ccc(C(F)(F)F)cc1</smiles>

The compound was prepared according to the General Procedure 1 using 1-bromo-4-(trifluoromethyl)benzene (45.0 mg, 0.2 mmol), mpg-CN (10.0 mg), $\mathrm{NiBr}_{2} \bullet$ glyme (3.2 mg, $5 \mathrm{~mol} \%$ ), 2,2'bipyridine $(1.6 \mathrm{mg} 5 \mathrm{~mol} \%), N, N$-dimethylacetamide $(1.0 \mathrm{~mL})$ and 2,6-lutidine $(70 \mu \mathrm{L} 0.6$ mmol). The reaction mixture was irradiated under nitrogen atmosphere for $48 \mathrm{~h}$ using blue LED $(455( \pm 15) \mathrm{nm})$. The reaction mixture was then subjected to the work-up procedure as described in the General Procedure 1. Purification was performed using flash chromatography on silica gel using PE/EtOAc solvent mixture as eluent to provide the title compound in 84\% yield (38.8 $\mathrm{mg}){ }^{1} \mathrm{H}$ NMR $\left(300 \mathrm{MHz}, \mathrm{CDCl}_{3}\right) \delta 7.62(\mathrm{~d}, J=8.2 \mathrm{~Hz}) \& 7.55(\mathrm{~d}, J=8.2 \mathrm{~Hz}, 2 \mathrm{H}), 7.34(\mathrm{~d}, J$ $=8.0 \mathrm{~Hz}) \& 7.28(\mathrm{~d}, J=8.0 \mathrm{~Hz}, 2 \mathrm{H}), 4.62(\mathrm{~s}) \& 4.57(\mathrm{~s}, 2 \mathrm{H}), 2.93(\mathrm{~s}, 3 \mathrm{H}), 2.16(\mathrm{~s}) \& 2.12(\mathrm{~s}$, $3 \mathrm{H}) .{ }^{13} \mathrm{C} \mathrm{NMR}\left(75 \mathrm{MHz}, \mathrm{CDCl}_{3}\right) \delta 171.1 \& 171.0,141.6(\mathrm{q}, J=1.3 \mathrm{~Hz}) \& 140.8(\mathrm{q}, J=1.3$ $\mathrm{Hz}), 130.0(\mathrm{q}, J=32.7 \mathrm{~Hz}) \& 129.9(\mathrm{q}, J=32.7 \mathrm{~Hz}), 128.2 \& 127.9,126.0(\mathrm{q}, J=3.6 \mathrm{~Hz}) \&$ $125.6(\mathrm{q}, J=3.6 \mathrm{~Hz}), 122.5(\mathrm{q}, J=270.1 \mathrm{~Hz}) \& 122.4(\mathrm{q}, J=270.1 \mathrm{~Hz}), 53.9 \& 50.4,35.8 \&$ 33.9, 21.8 \& 21.4. HRMS (ESI): $m / z$ calculated. for $\mathrm{C}_{11} \mathrm{H}_{13} \mathrm{~F}_{3} \mathrm{NO}[\mathrm{M}+\mathrm{H}]^{+}:$232.0944; found 232.0947.

\section{$N$-(4-fluorobenzyl)- $N$-methylacetamide (10)}

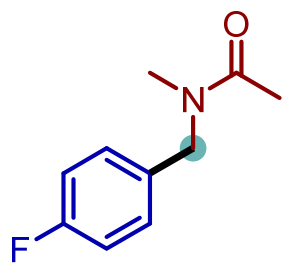

The compound was prepared according to the General Procedure 1 using 1-bromo-4-fluorobenzene (35.0 mg, $0.2 \mathrm{mmol})$, mpg-CN (10.0 mg), $\mathrm{NiBr} 2 \bullet$ glyme (3.2 mg, $5 \mathrm{~mol} \%$ ), 2,2'-bipyridine (1.6 mg $5 \mathrm{~mol} \%$ ), $N, N$ dimethylacetamide $(1.0 \mathrm{~mL})$ and 2,6-lutidine $(70 \mu \mathrm{L} 0.6 \mathrm{mmol})$. The reaction mixture was irradiated under nitrogen atmosphere for 48h using blue LED $(455( \pm 15)$ $\mathrm{nm})$. The reaction mixture was then subjected to the work-up procedure as described in the

General Procedure 1. Purification was performed using flash chromatography on silica gel using $\mathrm{PE} /$ EtOAc solvent mixture as eluent to provide the title compound in $76 \%$ yield $(27.5$ mg). ${ }^{1} \mathrm{H}$ NMR (300 MHz, $\left.\mathrm{CDCl}_{3}\right) \delta 7.24-7.09$ (m, 2H combined), $7.07-6.87$ (m, 2H, combined), 4.52 (s) \& 4.47 (s, $2 \mathrm{H}$ combined), 2.90 (s, 3H), 2.12 (s, 3H). ${ }^{13} \mathrm{C}$ NMR $(75 \mathrm{MHz}$, $\left.\mathrm{CDCl}_{3}\right) \delta 170.9 \& 170.7,162.3(\mathrm{~d}, J=245.4 \mathrm{~Hz}) \& 162.2(\mathrm{~d}, J=245.4 \mathrm{~Hz}), 133.3(\mathrm{~d}, J=3.5$ $\mathrm{Hz}) \& 132.3(\mathrm{~d}, J=3.5 \mathrm{~Hz}), 129.8(\mathrm{~d}, J=8.0 \mathrm{~Hz}) \& 128.08(\mathrm{~d}, J=8.0 \mathrm{~Hz}), 115.9(\mathrm{~d}, J=21.6$ $\mathrm{Hz}) \& 115.48(\mathrm{~d}, J=21.6 \mathrm{~Hz}) .53 .6 \& 50.0,35.5 \& 33.6,21.8 \& 21.48$. HRMS (ESI): $\mathrm{m} / \mathrm{z}$ calculated. for $\mathrm{C}_{10} \mathrm{H}_{13} \mathrm{FNO}[\mathrm{M}+\mathrm{H}]^{+}$: 182.0976; found 182.0977. 


\section{$N$-(4-bromobenzyl)- $N$-methylacetamide (11)}

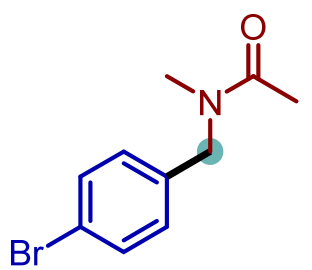

The compound was prepared according to the General Procedure 1 using 1,4-dibromobenzene (47.0 mg, $0.2 \mathrm{mmol}$ ), mpg-CN (10.0 mg), $\mathrm{NiBr}_{2} \bullet$ glyme (3.2 mg, $5 \mathrm{~mol} \%$ ), 2,2'-bipyridine (1.6 mg $5 \mathrm{~mol} \%$ ), $N, N$ dimethylacetamide $(1.0 \mathrm{~mL})$ and 2,6-lutidine $(70 \mu \mathrm{L} 0.6 \mathrm{mmol})$. The reaction mixture was irradiated under nitrogen atmosphere for 48h using blue LED $(455( \pm 15)$ $\mathrm{nm})$. The reaction mixture was then subjected to the work-up procedure as described in the General Procedure 1. Purification was performed using flash chromatography on silica gel using PE/EtOAc solvent mixture as eluent to provide the title compound in $72 \%$ yield $(34.8$ mg). ${ }^{1} \mathrm{H}$ NMR (400 MHz, $\left.\mathrm{CDCl}_{3}\right) \delta 7.49(\mathrm{~d}, J=8.3 \mathrm{~Hz}) \& 7.43(\mathrm{~d}, J=8.3 \mathrm{~Hz}, 2 \mathrm{H}$ combined), $7.12(\mathrm{~d}, J=8.3 \mathrm{~Hz}) \& 7.04(\mathrm{~d}, J=8.3 \mathrm{~Hz}, 2 \mathrm{H}$ combined), 4.52 (s) \& 4.47 (s, 2H combined), $2.92(\mathrm{~s}) \& 2.91\left(\mathrm{~s}, 3 \mathrm{H}\right.$ combined), $2.14(\mathrm{~s}) \& 2.13(\mathrm{~s}, 3 \mathrm{H}) .{ }^{13} \mathrm{C} \mathrm{NMR}\left(101 \mathrm{MHz}, \mathrm{CDCl}_{3}\right) \delta 171.0$ $\& 170.8,136.5 \& 135.7,132.2 \& 131.8,129.9 \& 128.1,121.6 \& 121.3,53.8 \& 50.2,35.6 \&$ 33.8, $21.9 \&$ 21.5. HRMS (APCI): $m / z$ calculated. for $\mathrm{C}_{10} \mathrm{H}_{13} \mathrm{BrNO}[\mathrm{M}+\mathrm{H}]^{+}: 242.0175$; found 242.0174 .

\section{$N$-methyl- $N$-(4-methylbenzyl)acetamide (12)}<smiles>CC(=O)N(C)Cc1ccc(C)cc1</smiles>

The compound was prepared according to the General Procedure 1 using 1-bromo-4-methylbenzene (34.2 mg, $0.2 \mathrm{mmol})$, mpg-CN (10.0 mg), $\mathrm{NiBr}_{2} \bullet$ glyme (3.2 mg, $5 \mathrm{~mol} \%$ ), 2,2'-bipyridine (1.6 mg $5 \mathrm{~mol} \%$ ), $N, N$ dimethylacetamide $(1.0 \mathrm{~mL})$ and 2,6-lutidine $(70 \mu \mathrm{L} 0.6 \mathrm{mmol})$. The reaction mixture was irradiated under nitrogen atmosphere for 48h using blue LED (455 $( \pm 15)$ $\mathrm{nm})$. The reaction mixture was then subjected to the work-up procedure as described in the

General Procedure 1. Purification was performed using flash chromatography on silica gel using $\mathrm{PE} /$ EtOAc solvent mixture as eluent to provide the title compound in $67 \%$ yield (23.7 $\mathrm{mg}$ ). Following the General Procedure 2 the same compound was synthesized from 1-chloro4-methylbenzene (25.2 mg, $0.2 \mathrm{mmol})$, mpg-CN (10.0 mg), NiCl$\bullet \bullet \cdot g l y m e ~(2.2 \mathrm{mg}, 5 \mathrm{~mol} \%$ ), 2,2'-bipyridine (1.6 mg $5 \mathrm{~mol} \%), N, N$-dimethylacetamide $(1.0 \mathrm{~mL})$ and 2,6-lutidine (70 $\mu \mathrm{L} 0.6$ mmol) with an isolated yield of $84 \%(29.7 \mathrm{mg}) .{ }^{1} \mathrm{H} \mathrm{NMR}\left(400 \mathrm{MHz}, \mathrm{CDCl}_{3}\right) \delta 7.39$ (s) \& 7.26 (s, 2H combined), $7.30(\mathrm{~d}, J=8.0 \mathrm{~Hz}) \& 7.19(\mathrm{~d}, J=8.0 \mathrm{~Hz}, 2 \mathrm{H}$ combined), 4.67 (s) \& 4.61 (s, $2 \mathrm{H}$ combined), 3.05 (s) \& 3.03 (s, 3H combined), 2.48 (s) \& 2.46 (s, 3H combined), 2.28 (s), 2.27 (s, 3H combined). ${ }^{13} \mathrm{C} \mathrm{NMR}\left(101 \mathrm{MHz}, \mathrm{CDCl}_{3}\right) \delta 171.1 \& 170.7,137.5 \& 137.1,134.4 \&$ $133.6,129.7 \& 129.3,128.2 \& 126.4,54.1 \& 50.4,35.5 \& 33.7,22.0 \& 21.6,21.2 \& 21.1$. EI-MS: $m / z$ calculated. for $\mathrm{C}_{11} \mathrm{H}_{15} \mathrm{NO}[\mathrm{M}]^{+}:$177.11482; found 177.11474 . 


\section{$N$-([1,1'-biphenyl]-4-ylmethyl)- $N$-methylacetamide (13)}<smiles>CC(=O)N(C)Cc1ccc(-c2ccccc2)cc1</smiles>

The compound was prepared according to the General Procedure 1 using 4-bromo-1,1'-biphenyl (46.6 mg, $0.2 \mathrm{mmol}$ ), mpg-CN (10.0 mg), NiBr $\bullet \bullet$ glyme (3.2 mg, 5 mol\%), 2,2'-bipyridine (1.6 mg $5 \mathrm{~mol} \%), N, N$-dimethylacetamide $(1.0 \mathrm{~mL})$ and 2,6-lutidine $(70 \mu \mathrm{L} 0.6 \mathrm{mmol})$. The reaction mixture was irradiated under nitrogen atmosphere for 48h using blue LED $(455( \pm 15)$ $\mathrm{nm})$. The reaction mixture was then subjected to the work-up procedure as described in the General Procedure 1. Purification was performed using flash chromatography on silica gel using $\mathrm{PE} / \mathrm{EtOAc}$ solvent mixture as eluent to provide the title compound in $77 \%$ yield (36.8 mg). ${ }^{1} \mathrm{H}$ NMR (300 MHz, $\left.\mathrm{CDCl}_{3}\right) \delta 7.64-7.51$ (m, 4H combined), $7.49-7.39$ (m, 2H combined), 7.39 - 7.20 (m, 3H combined), 4.63 (s) \& 4.56 (s, 2H combined), 2.98 (s) \& 2.96 (s, 3H combined), 2.18 (s) \& 2.17 (s, 3H combined). ${ }^{13} \mathrm{C}$ NMR $\left(75 \mathrm{MHz}, \mathrm{CDCl}_{3}\right) \delta 171.0 \&$ $170.7,140.8 \& 140.7,140.5 \& 140.4,136.5 \& 135.6,128.9 \& 128.8,128.5,127.74 \& 127.5$, $127.3 \& 127.3,127.1 \& 126.8,54.0 \& 50.4,35.6 \& 33.8,21.9 \& 21.5$. HRMS (ESI): $\mathrm{m} / \mathrm{z}$ calculated. for $\mathrm{C}_{16} \mathrm{H}_{18} \mathrm{NO}[\mathrm{M}+\mathrm{H}]^{+}: 240.1383$; found 240.1386 .

\section{$N$-methyl- $N$-(4-(4,4,5,5-tetramethyl-1,3,2-dioxaborolan-2-yl)benzyl)acetamide (14)}

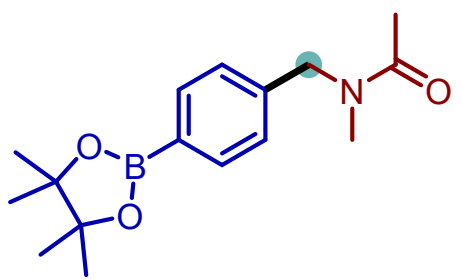

The compound was prepared according to the General

Procedure 1 using 2-(4-bromophenyl)-4,4,5,5-tetramethyl-1,3,2dioxaborolane (56.4 mg, $0.2 \mathrm{mmol})$, mpg-CN (10.0 mg), $\mathrm{NiBr} \bullet$ glyme (3.2 mg, $5 \mathrm{~mol} \%$ ), 2,2'-bipyridine (1.6 mg $5 \mathrm{~mol} \%$ ), $N$, $N$-dimethylacetamide $(1.0 \mathrm{~mL})$ and 2,6-lutidine $(70 \mu \mathrm{L} 0.6 \mathrm{mmol})$. The reaction mixture was irradiated under nitrogen atmosphere for 48h using blue LED (455 $( \pm 15) \mathrm{nm})$. The reaction mixture was then subjected to the work-up procedure as described in the General Procedure 1. Purification was performed using flash chromatography on silica gel using PE/EtOAc solvent mixture as eluent to provide the title compound in $75 \%$ yield $(43.3 \mathrm{mg}) .{ }^{1} \mathrm{H}$ NMR $(300 \mathrm{MHz}$, $\left.\mathrm{CDCl}_{3}\right) \delta 7.79(\mathrm{~d}, J=8.0 \mathrm{~Hz}) \& 7.75(\mathrm{~d}, J=8.0 \mathrm{~Hz}, 2 \mathrm{H}$ combined $), 7.22(\mathrm{~d}, J=8.0 \mathrm{~Hz}) \& 7.16$ (d, $J=8.0 \mathrm{~Hz}, 2 \mathrm{H}$ combined), 4.58 (s) \& 4.52 (s, 2H), 2.92 (s) \& 2.88 (s, 3H combined), 2.14 (s) \& 2.12 (s, 3H combined), 1.33 (s) \& 1.32 (s, $12 \mathrm{H}$ combined). ${ }^{13} \mathrm{C} \mathrm{NMR}\left(75 \mathrm{MHz}, \mathrm{CDCl}_{3}\right)$ $\delta 171.1 \& 170.7,140.6 \& 139.8,135.5 \& 135.1,127.4 \& 125.7,83.9 \& 83.8,54.4 \& 50.7,35.5$ \& 33.8, 24.9, 21.8 \& 21.4. HRMS (ESI): $\mathrm{m} / \mathrm{z}$ calculated. for $\mathrm{C}_{16} \mathrm{H}_{25} \mathrm{BNO}_{3}[\mathrm{M}+\mathrm{H}]^{+}:$289.1958; found 289.1962 . 


\section{$N$-methyl- $N$-(4-(trifluoromethoxy)benzyl)acetamide (15)}<smiles>CC(=O)N(C)Cc1ccc(OC(F)(F)F)cc1</smiles>

The compound was prepared according to the General Procedure 1 using 1-bromo-4-(trifluoromethoxy)benzene (48.2 mg, $0.2 \mathrm{mmol})$, mpg-CN (10.0 mg), $\mathrm{NiBr}_{2} \bullet$ glyme (3.2 mg, $5 \mathrm{~mol} \%$ ), 2,2'-bipyridine (1.6 mg 5 mol\%), $N, N$-dimethylacetamide $(1.0 \mathrm{~mL})$ and 2,6-lutidine $(70 \mu \mathrm{L} 0.6 \mathrm{mmol})$. The reaction mixture was irradiated under nitrogen atmosphere for 48h using blue LED $(455( \pm 15) \mathrm{nm})$. The reaction mixture was then subjected to the work-up procedure as described in the General Procedure 1. Purification was performed using flash chromatography on silica gel using $\mathrm{PE} /$ EtOAc solvent mixture as eluent to provide the title compound in $68 \%$ yield $(38.6 \mathrm{mg}) .{ }^{1} \mathrm{H}$ NMR (400 MHz, $\left.\mathrm{CDCl}_{3}\right) \delta 7.46-6.32(\mathrm{~m}, 4 \mathrm{H}), 4.56$ (s) \& 4.51 (s, 2H combined), $2.92(\mathrm{~s}, 3 \mathrm{H})$, 2.14 (s) \& 2.13 (s, 3H combined). $\left.{ }^{13} \mathrm{C} \mathrm{NMR} \mathrm{(101} \mathrm{MHz,} \mathrm{CDCl}_{3}\right) \delta 171.0 \& 170.9,148.7 \&$ $148.5,136.3 \& 135.4,129.4 \& 127.7,121.6 \& 121.2,120.5$ (q, $J=256.9 \mathrm{~Hz}), 53.6 \& 50.1,35.7$ \& 33.7, 21.8 \& 21.4. HRMS (APCI): $\mathrm{m} / \mathrm{z}$ calculated. for $\mathrm{C}_{11} \mathrm{H}_{13} \mathrm{~F}_{3} \mathrm{NO}_{2}[\mathrm{M}+\mathrm{H}]^{+}:$248.0893; found 248.0898 .

\section{$N$-methyl- $N$-(4-((trifluoromethyl)thio)benzyl)acetamide (16)}

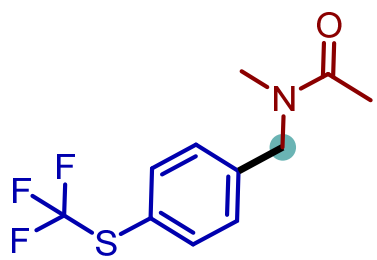

The compound was prepared according to the General Procedure 1 using (4-bromophenyl)(trifluoromethyl)sulfane (51.4 mg, $0.2 \mathrm{mmol})$, mpg-CN (10.0 mg), NiBr2•glyme (3.2 mg, $5 \mathrm{~mol} \%$ ), 2,2'-bipyridine (1.6 mg $5 \mathrm{~mol} \%), N, N$-dimethylacetamide $(1.0 \mathrm{~mL})$ and 2,6-lutidine ( $70 \mu \mathrm{L} 0.6 \mathrm{mmol})$. The reaction mixture was irradiated under nitrogen atmosphere for $48 \mathrm{~h}$ using blue LED $(455( \pm 15) \mathrm{nm})$. The reaction mixture was then subjected to the workup procedure as described in the General Procedure 1. Purification was performed using flash chromatography on silica gel using $\mathrm{PE} / \mathrm{EtOAc}$ solvent mixture as eluent to provide the title compound in $83 \%$ yield $(43.6 \mathrm{mg}) .{ }^{1} \mathrm{H}$ NMR $\left(400 \mathrm{MHz}, \mathrm{CDCl}_{3}\right) \delta 7.63(\mathrm{~d}, J=8.1 \mathrm{~Hz}) \& 7.57$ (d, $J=8.1 \mathrm{~Hz}, 2 \mathrm{H}$ combined), $7.26(\mathrm{~d}, J=8.1 \mathrm{~Hz}) \& 7.21(\mathrm{~d}, J=8.1 \mathrm{~Hz}, 2 \mathrm{H}$ combined), 4.58 (s) \& $4.54(\mathrm{~s}, 2 \mathrm{H}$ combined), 2.93 (s) \& 2.92 (s, 3H combined), 2.14 (s) \& 2.11 (s, 3H combined). ${ }^{13} \mathrm{C} \mathrm{NMR}\left(101 \mathrm{MHz}, \mathrm{CDCl}_{3}\right) \delta 171.0 \& 170.9,140.7 \& 140.0,136.9 \& 136.6,129.6$ $(\mathrm{q}, J=308.1 \mathrm{~Hz}) \& 129.5(\mathrm{q}, J=308.1 \mathrm{~Hz}), 128.9 \& 128.1,123.72(\mathrm{q}, J=4.2 \mathrm{~Hz}) \& 123.2(\mathrm{~d}$, $J=4.2 \mathrm{~Hz}$ ) $53.8 \& 50.3,35.9 \& 33.9,21.7 \&, 21.4$. HRMS (APCI): $\mathrm{m} / \mathrm{z}$ calculated. for $\mathrm{C}_{11} \mathrm{H}_{13} \mathrm{~F}_{3} \mathrm{NOS}[\mathrm{M}+\mathrm{H}]^{+}:$264.0664; found 264.0666. 


\section{$N$-(4-methoxybenzyl)- $N$-methylacetamide (17)}<smiles></smiles>

The compound was prepared according to the General Procedure 1 using 1-bromo-4-methoxybenzene (37.4 mg, $0.2 \mathrm{mmol}$ ), mpg-CN (10.0 mg), NiBr2•glyme (3.2 mg, 5 mol\%), 2,2'-bipyridine (1.6 mg $5 \mathrm{~mol} \%), N$, $N$-dimethylacetamide $(1.0 \mathrm{~mL})$ and 2,6-lutidine $(70 \mu \mathrm{L} 0.6 \mathrm{mmol})$. The reaction mixture was irradiated under nitrogen atmosphere for 48h using blue LED $(455( \pm 15)$ $\mathrm{nm})$. The reaction mixture was then subjected to the work-up procedure as described in the General Procedure 1. Purification was performed using flash chromatography on silica gel using PE/EtOAc solvent mixture as eluent to provide the title compound in $84 \%$ yield (32.4 mg). ${ }^{1} \mathrm{H}$ NMR (300 MHz, $\left.\mathrm{CDCl}_{3}\right) \delta 7.17$ (d, $\left.J=8.5 \mathrm{~Hz}\right) \& 7.08$ (d, $J=8.5 \mathrm{~Hz}, 2 \mathrm{H}$ combined), $6.88(\mathrm{~d}, J=8.5 \mathrm{~Hz}) \& 6.84(\mathrm{~d}, J=8.5 \mathrm{~Hz}, 2 \mathrm{H}$ combined), 4.51 (s) \& 4.44 (s, 2H combined), $3.80(\mathrm{~s}) \& 3.78$ (s, 3H combined), 2.90 (s) \& 2.89 (s, 3H combined), 2.15 (s) \& 2.12 (s, 3H combined). ${ }^{13} \mathrm{C}$ NMR (75 MHz, $\left.\mathrm{CDCl}_{3}\right) \delta 170.9 \& 170.6,159.3 \& 159.1,129.6 \& 129.5,128.6$ $\& 127.7,114.4 \& 114.1,55.3,53.8 \& 50.1,35.4 \& 33.5,21.95 \& 21.5$. HRMS (ESI): $m / z$ calculated. for $\mathrm{C}_{11} \mathrm{H}_{16} \mathrm{NO}_{2}[\mathrm{M}+\mathrm{H}]^{+}$: 194.1176; found 194.1179.

\section{$N$-methyl- $N$-(4-(methylthio)benzyl)acetamide (18)}<smiles>CSc1ccc(CN(C)C(C)=O)cc1</smiles>

mixture was irradiated under nitrogen atmosphere for $48 \mathrm{~h}$ using blue LED $(455( \pm 15) \mathrm{nm})$. The reaction mixture was then subjected to the work-up procedure as described in the General Procedure 1. Purification was performed using flash chromatography on silica gel using $\mathrm{PE} /$ EtOAc solvent mixture as eluent to provide the title compound in $66 \%$ yield $(27.6 \mathrm{mg}) .{ }^{1} \mathrm{H}$ NMR (400 MHz, $\left.\mathrm{CDCl}_{3}\right) \delta 7.37$ - $7.08(\mathrm{~m}, 4 \mathrm{H}), 4.57$ (s) \& 4.52 (s, 2H combined), 2.96 (s) \& 2.95 (s, 3H combined), 2.52 (s) \& 2.50 (s, 3H combined), $2.19(\mathrm{~s}, 3 \mathrm{H}) .{ }^{13} \mathrm{C}$ NMR $(101 \mathrm{MHz}$, $\left.\mathrm{CDCl}_{3}\right) \delta 171.0 \& 170.7,138.0 \& 137.5,134.3 \& 133.4,128.7 \& 127.1,126.9 \& 126.9,53.9 \&$ $50.2,35.5 \& 33.7,21.8 \& 21.5,16.0 \& 15.9$. HRMS (ESI): $\mathrm{m} / \mathrm{z}$ calculated. for $\mathrm{C}_{11} \mathrm{H}_{16} \mathrm{NOS}$ $[\mathrm{M}+\mathrm{H}]^{+}: 210.0947$; found 210.0948 . 


\section{$N$-(3,5-dimethoxybenzyl)- $N$-methylacetamide (19)}

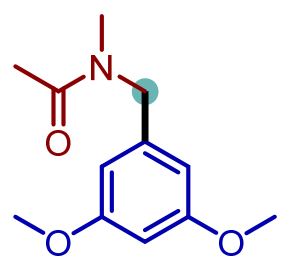

The compound was prepared according to the General Procedure 1 using 1-bromo-3,5-dimethoxybenzene (43.4 mg, $0.2 \mathrm{mmol}$ ), mpg-CN (10.0 $\mathrm{mg}$ ), $\mathrm{NiBr}_{2} \bullet$ glyme (3.2 mg, $5 \mathrm{~mol} \%$ ), 2,2'-bipyridine (1.6 mg $5 \mathrm{~mol} \%$ ), $N, N$ dimethylacetamide $(1.0 \mathrm{~mL})$ and 2,6-lutidine $(70 \mu \mathrm{L} 0.6 \mathrm{mmol})$. The reaction mixture was irradiated under nitrogen atmosphere for 48h using blue LED $(455( \pm 15) \mathrm{nm})$. The reaction mixture was then subjected to the work-up procedure as described in the General Procedure 1. Purification was performed using flash chromatography on silica gel using $\mathrm{PE} /$ EtOAc solvent mixture as eluent to provide the title compound in $81 \%$ yield $(36.1 \mathrm{mg}) .{ }^{1} \mathrm{H}$ NMR (400 MHz, $\left.\mathrm{CDCl}_{3}\right) \delta 6.42-6.20(\mathrm{~m}, 3 \mathrm{H}), 4.48(\mathrm{~s}) \& 4.42$ (s, 2H combined), 3.75 (s) \& 3.73 (s, 6H combined), 2.91 (s) \& 2.89 (s, 3H combined), 2.12 (s) \& 2.10 (s, 3H combined). ${ }^{13} \mathrm{C} \mathrm{NMR}\left(101 \mathrm{MHz}, \mathrm{CDCl}_{3}\right) \delta 171.1 \& 170.7,161.4 \& 161.0,139.8 \& 139.2,105.9 \& 104.2$, $99.2 \& 99.1,55.4 \& 55.3,54.3 \& 50.6,35.5 \& 33.8,21.8 \& 21.4$. HRMS (APCI): $m / z$ calculated. for $\mathrm{C}_{12} \mathrm{H}_{17} \mathrm{NO}_{3}[\mathrm{M}+\mathrm{H}]^{+}:$224.1281; found 224.1283.

\section{$N$-(3-acetylbenzyl)- $N$-methylacetamide (20)}<smiles></smiles>

The compound was prepared according to the General Procedure 1 using 1-(3-bromophenyl)ethan-1-one (39.8 mg, 0.2 mmol), mpg-CN (10.0 mg), NiBr $2 \bullet$ glyme (3.2 mg, $5 \mathrm{~mol} \%$ ), 2,2'bipyridine $(1.6 \mathrm{mg} 5 \mathrm{~mol} \%), N, N$-dimethylacetamide $(1.0 \mathrm{~mL})$ and 2,6-lutidine $(70 \mu \mathrm{L} 0.6$ mmol). The reaction mixture was irradiated under nitrogen atmosphere for $48 \mathrm{~h}$ using blue LED $(455( \pm 15) \mathrm{nm})$. The reaction mixture was then subjected to the work-up procedure as described in the General Procedure 1. Purification was performed using flash chromatography on silica gel using PE/EtOAc solvent mixture as eluent to provide the title compound in 79\% yield (32.4 mg). ${ }^{1} \mathrm{H}$ NMR (400 MHz, $\left.\mathrm{CDCl}_{3}\right) \delta 7.88-7.69(\mathrm{~m}, 2 \mathrm{H}), 7.50-7.31$ (m, 2H), $4.60(\mathrm{~s}) \& 4.55$ (s, $2 \mathrm{H}$ combined), 2.92 (s) \& 2.91 (s, 3H combined), 2.57 (s) \& 2.56 (s, 3H combined), 2.14 (s) $\& 2.12$ (s, 3H combined). ${ }^{13} \mathrm{C}$ NMR $(101 \mathrm{MHz}, \mathrm{CDCl} 3) \delta 198.0 \& 197.7,171.0 \& 170.9,138.1$ $\& 137.8,137.5 \& 137.4,132.7 \& 130.8,129.3 \& 129.0,127.8 \& 127.6,127.5 \& 126.1,54.0 \&$ 50.5, $35.7 \& 33.7,26.7,21.8 \&$ 21.5. EI-MS: $m / z$ calculated. for $\mathrm{C}_{12} \mathrm{H}_{15} \mathrm{NO}_{2}[\mathrm{M}]^{+}:$205.10973; found 205.11.013. 


\section{$N$-methyl- $N$-(3-(trifluoromethyl)benzyl)acetamide (21)}<smiles>CC(=O)N(C)Cc1cccc(C(F)(F)F)c1</smiles>

The compound was prepared according to the General Procedure 1 using 1-bromo-3-(trifluoromethyl)benzene (45.0 mg, 0.2 mmol), mpg-CN (10.0 mg), $\mathrm{NiBr}_{2} \bullet$ glyme (3.2 mg, $5 \mathrm{~mol} \%$ ), 2,2'bipyridine $(1.6 \mathrm{mg} 5 \mathrm{~mol} \%), N, N$-dimethylacetamide $(1.0 \mathrm{~mL})$ and 2,6-lutidine $(70 \mu \mathrm{L} 0.6$ mmol). The reaction mixture was irradiated under nitrogen atmosphere for $48 \mathrm{~h}$ using blue LED $(455( \pm 15) \mathrm{nm})$. The reaction mixture was then subjected to the work-up procedure as described in the General Procedure 1. Purification was performed using flash chromatography on silica gel using PE/EtOAc solvent mixture as eluent to provide the title compound in $82 \%$ yield (37.8 mg). ${ }^{1} \mathrm{H}$ NMR (400 MHz, $\left.\mathrm{CDCl}_{3}\right) \delta 7.59-7.33$ (m, 4H), 4.63 (s) \& 4.58 (s, 2H combined), 2.95 (s, 3H), 2.17 (s) \& 2.15 (s, 3H combined). ${ }^{13} \mathrm{C}$ NMR $(101 \mathrm{MHz}, \mathrm{CDCl} 3) \delta 171.0 \& 171.9$, $138.4 \& 137.7,131.1(\mathrm{q}, J=32.1 \mathrm{~Hz}) \& 131.0(\mathrm{q}, J=32.1 \mathrm{~Hz}), 131.3 \& 129.1,129.6 \& 129.5$, $124.6(\mathrm{q}, J=3.5 \mathrm{~Hz}), 124.3(\mathrm{q}, J=3.5 \mathrm{~Hz}), 123.2(\mathrm{q}, J=270.1 \mathrm{~Hz}), 53.9 \& 50.4,35.7 \& 33.8$, 21.8 \& 21.4. EI-MS: $m / z$ calculated. for $\mathrm{C}_{11} \mathrm{H}_{12} \mathrm{NOF}_{3}[\mathrm{M}]^{+}: 231.08655$; found 231.08620.

\section{$N$-methyl- $N$-(2-methylbenzyl)acetamide (22)}<smiles>CC(=O)N(C)Cc1ccccc1C</smiles>

The compound was prepared according to the General Procedure 1 using 1-bromo-2-methylbenzene (34.2 mg, $0.2 \mathrm{mmol})$, mpg-CN (10.0 mg), $\mathrm{NiBr} 2 \bullet$ glyme $(3.2 \mathrm{mg}, 5 \mathrm{~mol} \%), 2,2^{\prime}$-bipyridine (1.6 mg $\left.5 \mathrm{~mol} \%\right), N, N$ dimethylacetamide $(1.0 \mathrm{~mL})$ and 2,6-lutidine $(70 \mu \mathrm{L} 0.6 \mathrm{mmol})$. The reaction mixture was irradiated under nitrogen atmosphere for 48h using blue LED (455 $( \pm 15) \mathrm{nm})$. The reaction mixture was then subjected to the work-up procedure as described in the General Procedure 1. Purification was performed using flash chromatography on silica gel using PE/EtOAc solvent mixture as eluent to provide the title compound in $73 \%$ yield $(25.8 \mathrm{mg})$. Following the General Procedure 2 the same compound was synthesized from 1-chloro-2-methylbenzene $(25.2 \mathrm{mg}$, $0.2 \mathrm{mmol})$, mpg-CN (10.0 mg), $\mathrm{NiCl}_{2} \bullet$ glyme (2.2 mg, $\left.5 \mathrm{~mol} \%\right), 2,2^{\prime}$-bipyridine (1.6 mg 5 mol\%), $N, N$-dimethylacetamide $(1.0 \mathrm{~mL})$ and 2,6-lutidine $(70 \mu \mathrm{L} 0.6 \mathrm{mmol})$ with an isolated yield of $81 \%$ (28.6 mg). ${ }^{1} \mathrm{H}$ NMR (400 MHz, $\left.\mathrm{CDCl}_{3}\right) \delta 7.24-7.01(\mathrm{~m}, 4 \mathrm{H}), 4.61(\mathrm{~s}) \& 4.47$ (s, 2H combined), 2.96 (s) \& 2.88 (s, 3H combined), 2.28 (s, 3H), 2.17 (s) \& 2.09 (s, 3H combined). ${ }^{13} \mathrm{C}$ NMR $\left(101 \mathrm{MHz}, \mathrm{CDCl}_{3}\right) \delta 171.4 \& 170.7,136.6 \& 135.3,134.9 \& 134.3,130.7 \& 130.6$, $128.0 \& 127.5,127.4 \& 126.6,126.1 \& 125.1,52.2 \& 48.4,35.4 \& 34.0,21.9 \& 21.4,19.2 \&$ 19.0. EI-MS: $m / z$ calculated. for $\mathrm{C}_{11} \mathrm{H}_{15} \mathrm{NO}[\mathrm{M}]^{+}:$177.11482; found 177.11500 . 


\section{Methyl 2-(( $N$-methylacetamido)methyl)benzoate (23)}<smiles>COC(=O)c1ccccc1CN(C)C(C)=O</smiles>

The compound was prepared according to the General Procedure 1 using methyl 2-bromobenzoate (43.0 mg, $0.2 \mathrm{mmol}), \mathrm{mpg}-\mathrm{CN}(10.0 \mathrm{mg})$, $\mathrm{NiBr}_{2} \bullet$ glyme (3.2 mg, $5 \mathrm{~mol} \%$ ), 2,2'-bipyridine (1.6 mg $\left.5 \mathrm{~mol} \%\right), \mathrm{N}, \mathrm{N}$ dimethylacetamide $(1.0 \mathrm{~mL})$ and 2,6-lutidine $(70 \mu \mathrm{L} 0.6 \mathrm{mmol})$. The reaction mixture was irradiated under nitrogen atmosphere for 48h using blue LED (455 $( \pm 15) \mathrm{nm})$. The reaction mixture was then subjected to the work-up procedure as described in the General Procedure 1. Purification was performed using flash chromatography on silica gel using PE/EtOAc solvent mixture as eluent to provide the title compound in $46 \%$ yield (20.3 mg). ${ }^{1} \mathrm{H}$ NMR (400 MHz, $\left.\mathrm{CDCl}_{3}\right) \delta 8.04(\mathrm{dd}, J=7.8,1.0 \mathrm{~Hz}) \& 7.92(\mathrm{dd}, J=7.8,1.0 \mathrm{~Hz} 1 \mathrm{H}$ combined), $7.54(\mathrm{td}, J=7.6,1.2 \mathrm{~Hz}) \& 7.47(\mathrm{td}, J=7.6,1.2 \mathrm{~Hz}, 1 \mathrm{H}$ combined), $7.36(\mathrm{td}, J=$ 7.6, $1.2 \mathrm{~Hz}) \& 7.30(\mathrm{td}, J=7.6,1.2 \mathrm{~Hz}, 1 \mathrm{H}$ combined), 5.00 (s) \& 4.94 (s, 2H combined), 3.90 (s) \& $3.88(\mathrm{~s}, 3 \mathrm{H}), 2.98(\mathrm{~s}) \& 2.97\left(\mathrm{~s}, 3 \mathrm{H}\right.$ combined), $2.19(\mathrm{~s}) \& 2.04\left(\mathrm{~s}, 3 \mathrm{H}\right.$ combined). ${ }^{13} \mathrm{C}$ NMR $\left(101 \mathrm{MHz}, \mathrm{CDCl}_{3}\right) \delta 171.8 \& 171.1,167.8 \& 167.3,139.1 \& 139.0,133.20 \& 132.5$, $131.6 \& 130.9,129.3 \& 128.2,127.6 \& 127.3,126.9 \& 125.8,53.3 \& 49.0,52.2 \& 52.1,36.3$ \& 34.3, 21.9 \& 21.3. EI-MS: $\mathrm{m} / \mathrm{z}$ calculated. for $\mathrm{C}_{12} \mathrm{H}_{15} \mathrm{NO}_{3}[\mathrm{M}]^{+}: 221.10464$; found 221.10447 .

\section{$N$-(2-cyanobenzyl)- $N$-methylacetamide (24)}

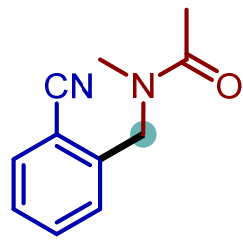

The compound was prepared according to the General Procedure 1 using methyl 2-bromobenzonitrile (36.4 mg, $0.2 \mathrm{mmol})$, mpg-CN (10.0 mg), $\mathrm{NiBr} 2 \bullet$ glyme (3.2 mg, $5 \mathrm{~mol} \%$ ), 2,2'-bipyridine (1.6 mg $5 \mathrm{~mol} \%), \mathrm{N}, \mathrm{N}$ dimethylacetamide $(1.0 \mathrm{~mL})$ and 2,6-lutidine $(70 \mu \mathrm{L} 0.6 \mathrm{mmol})$. The reaction mixture was irradiated under nitrogen atmosphere for $48 \mathrm{~h}$ using blue LED $(455( \pm 15) \mathrm{nm})$. The reaction mixture was then subjected to the work-up procedure as described in the General Procedure 1. Purification was performed using flash chromatography on silica gel using $\mathrm{PE} /$ EtOAc solvent mixture as eluent to provide the title compound in $69 \%$ yield $(25.9 \mathrm{mg}) .{ }^{1} \mathrm{H}$ NMR (400 MHz, $\left.\mathrm{CDCl}_{3}\right) \delta 7.71-7.49(\mathrm{~m}, 2 \mathrm{H}), 7.44-7.20(\mathrm{~m}, 2 \mathrm{H}), 4.79$ (s) \& $4.73(\mathrm{~s}, 2 \mathrm{H}$ combined), 3.00 (s) \& 2.94 (s, 3H combined), 2.16 (s) \& 2.11 (s, 3H combined). ${ }^{13} \mathrm{C}$ NMR (101 $\left.\mathrm{MHz}, \mathrm{CDCl}_{3}\right) \delta 171.2 \& 171.1,141.3 \& 140.4,133.6 \& 133.4,133.31 \& 132.8,128.8 \& 128.3$, $127.9 \& 126.4,117.6 \& 116.9,111.8 \& 111.1,52.5 \& 48.9,36.2 \& 33.9,21.7 \& 21.4$ EI-MS: $m / z$ calculated. for $\mathrm{C}_{11} \mathrm{H}_{12} \mathrm{~N}_{2} \mathrm{O}[\mathrm{M}]^{+}:$188.09441; found 188.09437 . 
<smiles>CC(=O)N(C)Cc1cc(Br)cc(C#N)c1</smiles>

The compound was prepared according to the General Procedure 1 using 3,5-dibromobenzonitrile (52.0 mg, $0.2 \mathrm{mmol}$ ), mpg-CN (10.0 mg), NiBr $\bullet$ glyme (3.2 mg, 5 mol\%), 2,2'-bipyridine (1.6 mg $5 \mathrm{~mol} \%), N, N$-dimethylacetamide $(1.0 \mathrm{~mL})$ and 2,6-lutidine $(70 \mu \mathrm{L}$ $0.6 \mathrm{mmol})$. The reaction mixture was irradiated under nitrogen atmosphere for $48 \mathrm{~h}$ using blue LED $(455( \pm 15) \mathrm{nm})$. The reaction mixture was then subjected to the work-up procedure as described in the General Procedure 1. Purification was performed using flash chromatography on silica gel using PE/EtOAc solvent mixture as eluent to provide the title compound in $67 \%$ yield (35.7 mg). ). ${ }^{1} \mathrm{H}$ NMR (400 MHz, $\left.\mathrm{CDCl}_{3}\right) \delta 7.72$ (s) \& 7.67 (s, 1 H combined), 7.61 (s) \& 7.54 (s, $1 \mathrm{H}$ combined), 7.45 (s) \& 7.40 (s, 1H combined), 4.55 (s) \& 4.53 (s, 2H combined), 2.97 (s) \& 2.93 (s, 3H combined), 2.17 (s) \& 2.12 (s, 3H combined). ${ }^{13} \mathrm{C}$ NMR (101 MHz, $\left.\mathrm{CDCl}_{3}\right) \delta 171.1 \& 170.8,141.3 \& 140.6,135.5 \& 134.1,133.8 \& 133.7,130.0 \& 128.5,123.8$ $\& 123.2,117.2, \& 116.9,114.9 \& 114.4,53.22 \& 49.9,36.1 \& 33.9,21.7 \&$ 21.5. HRMS (APCI): $m / z$ calculated. for $\mathrm{C}_{11} \mathrm{H}_{12} \mathrm{BrN}_{2} \mathrm{O}[\mathrm{M}+\mathrm{H}]^{+}: 267.0128$; found 267.0128.

\section{$N$-(3,5-dibromobenzyl)- $N$-methylacetamide (26)}<smiles>CC(=O)N(C)Cc1cc(Br)cc(Br)c1</smiles>

The compound was prepared according to the General Procedure 1 using 1,3,5-tribromobenzene (62.8 mg, $0.2 \mathrm{mmol})$, mpg-CN (10.0 mg), NiBr2•glyme (3.2 mg, 5 mol\%), 2,2'-bipyridine (1.6 $\mathrm{mg} 5 \mathrm{~mol} \%), N, N$-dimethylacetamide $(1.0 \mathrm{~mL})$ and 2,6-lutidine $(70 \mu \mathrm{L}$

$0.6 \mathrm{mmol})$. The reaction mixture was irradiated under nitrogen atmosphere for $48 \mathrm{~h}$ using blue LED $(455( \pm 15) \mathrm{nm})$. The reaction mixture was then subjected to the work-up procedure as described in the General Procedure 1. Purification was performed using flash chromatography on silica gel using PE/EtOAc solvent mixture as eluent to provide the title compound in $63 \%$ yield (40.4 mg). ${ }^{1} \mathrm{H}$ NMR (400 MHz, $\left.\mathrm{CDCl}_{3}\right) \delta 7.62-7.53(\mathrm{~m}, 1 \mathrm{H}), 7.34-7.20(\mathrm{~m}, 2 \mathrm{H}), 4.52$ (s), 4.47 (s, 2H combined), 2.95 (s), 2.94 (s, 3H combined), 2.17 (s) \& 2.13 (s, 3H combined). ${ }^{13} \mathrm{C}$ NMR $\left(101 \mathrm{MHz}, \mathrm{CDCl}_{3}\right) \delta 171.0 \& 170.9,141.6 \& 140.8,133.6 \& 133.2,129.8 \& 128.2$, $123.8 \& 123.3,53.3 \& 49.9,35.9 \& 34.0,21.8 \& 21.5$. HRMS (APCI): $m / z$ calculated. for $\mathrm{C}_{10} \mathrm{H}_{12} \mathrm{Br}_{2} \mathrm{NO}[\mathrm{M}+\mathrm{H}]^{+}: 319.928$; found 319.928. 
<smiles>CC(=O)N(C)Cc1ccc(C2(CN)CC2)cc1</smiles>

The compound was prepared according to the General Procedure 1 using 1-(4-bromophenyl)cyclopropane-1-carbonitrile (44.4 mg, $0.2 \mathrm{mmol})$, mpg-CN (10.0 mg), NiBr $\bullet$ glyme (3.2 mg, 5 mol\%), 2,2'-bipyridine (1.6 mg 5 mol\%), $N, N$-dimethylacetamide (1.0 atmosphere for $48 \mathrm{~h}$ using blue LED $(455( \pm 15) \mathrm{nm})$. The reaction mixture was then subjected to the work-up procedure as described in the General Procedure 1. Purification was performed using flash chromatography on silica gel using PE/EtOAc solvent mixture as eluent to provide the title compound in $82 \%$ yield $(37.3 \mathrm{mg}) .{ }^{1} \mathrm{H}$ NMR (300 MHz, $\left.\mathrm{CDCl}_{3}\right) \delta 7.37-7.12(\mathrm{~m}, 4 \mathrm{H})$, $4.58(\mathrm{~s}) \& 4.54(\mathrm{~s}, 2 \mathrm{H}$ combined), 2.95 (s, 3H), 2.18 (s) \& 2.16 (s, 3H combined), $1.82-1.65$ $(\mathrm{m}, 2 \mathrm{H}), 1.48-1.34(\mathrm{~m}, 2 \mathrm{H}) .{ }^{13} \mathrm{C} \mathrm{NMR}\left(75 \mathrm{MHz}, \mathrm{CDCl}_{3}\right) \delta 170.9 \& 170.8,137.0 \& 136.2$, $135.6 \& 135.1,128.5 \& 126.9,126.4 \& 126.0,122.5 \& 122.4,53.8 \& 50.1,35.6 \& 33.7,21.8$ \& 21.4, 18.2, 18.1, 13.5. HRMS (APCI): $\mathrm{m} / \mathrm{z}$ calculated. for $\mathrm{C}_{14} \mathrm{H}_{17} \mathrm{~N}_{2} \mathrm{O}[\mathrm{M}+\mathrm{H}]^{+}: 229.1335$; found 229.1343 .

\section{$N$-((1,3-dioxo-1H,3H-benzo[de]isochromen-6-yl)methyl)- $N$-methylacetamide (28)}<smiles>CC(=O)N(C)Cc1ccc2c3c(cccc13)C(=O)OC2=O</smiles>

The compound was prepared according to the General Procedure 1 using 6-bromo-1H,3H-benzo[de]isochromene-1,3-dione $(55.4 \mathrm{mg}, 0.2$

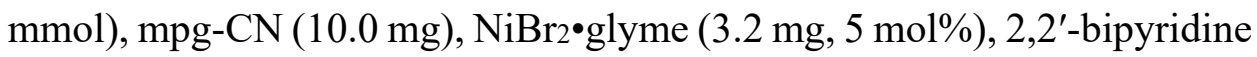
(1.6 mg $5 \mathrm{~mol} \%), N, N$-dimethylacetamide $(1.0 \mathrm{~mL})$ and 2,6-lutidine $(70 \mu \mathrm{L}$ $0.6 \mathrm{mmol})$. The reaction mixture was irradiated under nitrogen atmosphere for $48 \mathrm{~h}$ using blue LED $(455( \pm 15) \mathrm{nm})$. The reaction mixture was then subjected to the workup procedure as described in the General Procedure 1. Purification was performed using flash chromatography on silica gel using $\mathrm{PE} / \mathrm{EtOAc}$ solvent mixture as eluent to provide the title compound in $61 \%$ yield $(34.5 \mathrm{mg}) .{ }^{1} \mathrm{H}$ NMR $\left(400 \mathrm{MHz}, \mathrm{CDCl}_{3}\right) \delta 8.73-8.33(\mathrm{~m}, 3 \mathrm{H}), 7.99-$ $7.75(\mathrm{~m}, 1 \mathrm{H}), 7.74-7.53(\mathrm{~m}, 1 \mathrm{H}), 5.18(\mathrm{~s}) \& 5.15$ (s, 2H combined), 3.10 (s) \& 2.99 (s, 3H combined) 2.23 (s) \& 2.14 (s, $3 \mathrm{H}$ combined). ${ }^{13} \mathrm{C} \mathrm{NMR}\left(101 \mathrm{MHz}, \mathrm{CDCl}_{3}\right) \delta 171.4 \& 171.0$, $160.6 \& 160.4,142.5(x 2), 133.6 \& 133.5,133.5 \& 133.1,132.0(x 2), 130.8 \& 130.4,129.7 \&$ $129.4,128.1 \& 123.9,128.0 \& 127.6,119.3 \& 118.7,52.1 \& 48.6,35.7 \& 34.6,22.0 \& 21.4$. HRMS (APCI): $m / z$ calculated. for $\mathrm{C}_{16} \mathrm{H}_{14} \mathrm{NO}_{4}[\mathrm{M}+\mathrm{H}]^{+}: 284.0917$; found 284.0923. 
Ethyl 5-(( $N$-methylacetamido)methyl)benzofuran-2-carboxylate (29)

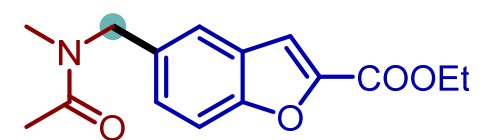

The compound was prepared according to the General

Procedure 3 using ethyl 5-bromobenzofuran-2-carboxylate (53.8 mg, $0.2 \mathrm{mmol}$ ), mpg-CN (10.0 mg), NiBr $2 \bullet g l y m e ~(3.2 \mathrm{mg}, 5$ mol\%), 2,2'-bipyridine (1.6 mg 5 mol\%), $N, N$-dimethylacetamide $(1.0 \mathrm{~mL})$ and 2,6-lutidine $(70 \mu \mathrm{L} 0.6 \mathrm{mmol})$. The reaction mixture was irradiated under nitrogen atmosphere for $48 \mathrm{~h}$ using blue LED $(455( \pm 15) \mathrm{nm})$. The reaction mixture was then subjected to the work-up procedure as described in the General Procedure 3. Purification was performed using flash chromatography on silica gel using $\mathrm{PE} /$ EtOAc solvent mixture as eluent to provide the title compound in $86 \%$ yield $(47.3 \mathrm{mg}) .{ }^{1} \mathrm{H}$ NMR (400 MHz, $\left.\mathrm{CDCl}_{3}\right) \delta 7.39-7.23(\mathrm{~m}, 3 \mathrm{H}), 7.14(\mathrm{dd}, J=8.6,1.6 \mathrm{~Hz}) \& 7.04(\mathrm{dd}, J=8.6$, $1.6 \mathrm{~Hz}, 1 \mathrm{H}$ combined), 4.45 (s) \& 4.41 (s, 2H combined), 4.23 (q, J=7.1 Hz) \& 4.22 (q, $J=$ $7.1 \mathrm{~Hz}, 2 \mathrm{H}$ combined), 2.74 (s) \& 2.73 (s, 3H combined), 1.97 (s) \& 1.96 (s, 3H combined), $1.22(\mathrm{t}, J=7.1 \mathrm{~Hz}), 1.21(\mathrm{t}, J=7.1 \mathrm{~Hz}, 3 \mathrm{H}$ combined $) .{ }^{13} \mathrm{C} \mathrm{NMR}\left(101 \mathrm{MHz}, \mathrm{CDCl}_{3}\right) \delta 171.0 \&$ $170.8,159.5 \& 159.4 \& 155.1,146.5 \& 146.2,133.3 \& 132.3,128.0 \& 127.5,127.2 \& 126.1$, $122.2 \& 120.2,113.6 \& 113.5,112.9 \& 112.5,61.7 \& 61.6,54.1 \& 50.5,35.6 \& 33.7,21.9 \&$ 21.5, 14.3. HRMS (APCI): $\mathrm{m} / \mathrm{z}$ calculated. for $\mathrm{C}_{15} \mathrm{H}_{17} \mathrm{NO}_{4}[\mathrm{M}+\mathrm{H}]^{+}: 276.1230$; found 276.1236.

\section{$N$-methyl- $N$-(thiophen-3-ylmethyl)acetamide (30)}

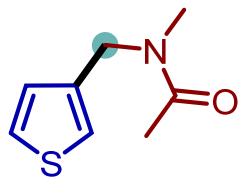

The compound was prepared according to the General Procedure 3 using 3bromothiophene (32.6 mg, $0.2 \mathrm{mmol})$, mpg-CN (10.0 mg), NiBr $\bullet$ glyme (3.2 $\mathrm{mg}, 5 \mathrm{~mol} \%$ ), 2,2'-bipyridine (1.6 mg $5 \mathrm{~mol} \%), N, N$-dimethylacetamide $(1.0$ $\mathrm{mL})$ and 2,6-lutidine $(70 \mu \mathrm{L} 0.6 \mathrm{mmol})$. The reaction mixture was irradiated under nitrogen atmosphere for $48 \mathrm{~h}$ using blue LED $(455( \pm 15) \mathrm{nm})$. The reaction mixture was then subjected to the work-up procedure as described in the General Procedure 3. Purification was performed using flash chromatography on silica gel using PE/EtOAc solvent mixture as eluent to provide the title compound in $60 \%$ yield $(20.3 \mathrm{mg}) .{ }^{1} \mathrm{H}$ NMR $\left(400 \mathrm{MHz}, \mathrm{CDCl}_{3}\right) \delta 7.33(\mathrm{dd}, J=5.0$, $3.0 \mathrm{~Hz}) \& 7.25(\mathrm{dd}, J=5.0,3.0 \mathrm{~Hz}, 1 \mathrm{H}$ combined $), 7.10(\mathrm{dd}, J=2.8,1.2 \mathrm{~Hz}) \& 7.04(\mathrm{dd}, J=$ 2.8, $1.2 \mathrm{~Hz}, 1 \mathrm{H}$ combined), 6.99 (dd, $J=4.9,1.1 \mathrm{~Hz}) \& 6.91$ (dd, $J=5.0,1.2 \mathrm{~Hz}, 1 \mathrm{H}$ combined), 4.53 (s) \& 4.48 (s, 3H combined), 2.94 (s) \& 2.92 (s, 3H combined), 2.14 (s) \& 2.10 (s, 3H combined). ${ }^{13} \mathrm{C} \mathrm{NMR}\left(101 \mathrm{MHz}, \mathrm{CDCl}_{3}\right) \delta 170.8 \& 170.5,138.2 \& 137.8,127.8 \& 127.0,126.2$ $\& 126.1,122.7 \& 121.5,50.3 \& 45.9,35.5 \& 33.7,21.9 \& 21.4$. HRMS (APCI): $\mathrm{m} / \mathrm{z}$ calculated. for $\mathrm{C}_{8} \mathrm{H}_{11} \mathrm{NOS}[\mathrm{M}+\mathrm{H}]^{+}:$170.0634; found 170.0635 . 


\section{$N$-((5-acetylthiophen-2-yl)methyl)- $N$-methylacetamide (31)}

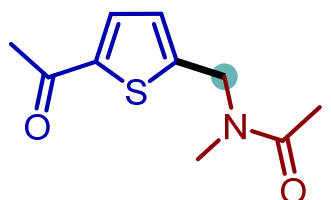

The compound was prepared according to the General Procedure 3 using 1-(5-bromothiophen-2-yl)ethan-1-one (41.0 mg, $0.2 \mathrm{mmol})$, mpg-CN

$(10.0 \mathrm{mg}), \mathrm{NiBr}_{2} \bullet$ glyme $(3.2 \mathrm{mg}, 5 \mathrm{~mol} \%), 2,2^{\prime}$-bipyridine (1.6 mg 5 mol\%), $N, N$-dimethylacetamide $(1.0 \mathrm{~mL})$ and 2,6-lutidine $(70 \mu \mathrm{L} 0.6 \mathrm{mmol})$. The reaction mixture was irradiated under nitrogen atmosphere for 48h using blue LED $(455( \pm 15) \mathrm{nm})$. The reaction mixture was then subjected to the work-up procedure as described in the General Procedure 3. Purification was performed using flash chromatography on silica gel using $\mathrm{PE} / \mathrm{EtOAc}$ solvent mixture as eluent to provide the title compound in $63 \%$ yield $(26.5 \mathrm{mg}) .{ }^{1} \mathrm{H}$ NMR (400 MHz, $\left.\mathrm{CDCl}_{3}\right) \delta 7.55(\mathrm{~d}, J=3.8 \mathrm{~Hz}) \& 7.52(\mathrm{~d}, J=3.8 \mathrm{~Hz}, 1 \mathrm{H}$ combined), 6.95 (d, $J=3.8 \mathrm{~Hz}) \& 6.91(\mathrm{~d}, J=3.8 \mathrm{~Hz}, 1 \mathrm{H}$ combined), 4.67 (s) \& 4.63 (s, 2H combined), 2.99 (s) \& $2.95\left(\mathrm{~s}, 3 \mathrm{H}\right.$ combined), $2.50(\mathrm{~s}) \& 2.48\left(\mathrm{~s}, 3 \mathrm{H}\right.$ combined), $2.15(\mathrm{~s}) \& 2.09$ (s, 3H combined). ${ }^{13} \mathrm{C}$ NMR $\left(101 \mathrm{MHz}, \mathrm{CDCl}_{3}\right) \delta 190.59 \& 190.4,170.6 \& 170.4,149.3 \& 149.1 \& 144.0 \& 143.7$, $132.6 \& 132.5,127.2 \& 126.0,50.0 \& 46.1,35.9 \& 33.7,26.6 \& 26.6,21.7 \&$ 21.4. HRMS (APCI): $m / z$ calculated. for $\mathrm{C}_{10} \mathrm{H}_{14} \mathrm{NO}_{2} \mathrm{~S}[\mathrm{M}+\mathrm{H}]^{+}: 212.0740$; found 212.0742 .

\section{$N$-(benzo[b]thiophen-5-ylmethyl)- $N$-methylacetamide (32)}<smiles>CC(=O)N(C)Cc1ccc2sccc2c1</smiles>

The compound was prepared according to the General Procedure 3 using 5-bromobenzo[b]thiophene (42.6 mg, $0.2 \mathrm{mmol})$, mpg-CN (10.0 $\mathrm{mg}), \mathrm{NiBr}_{2} \bullet \mathrm{glyme}(3.2 \mathrm{mg}, 5 \mathrm{~mol} \%), 2,2^{\prime}$-bipyridine (1.6 mg $5 \mathrm{~mol} \%$ ), $N, N$-dimethylacetamide $(1.0 \mathrm{~mL})$ and 2,6-lutidine $(70 \mu \mathrm{L} 0.6 \mathrm{mmol})$. The reaction mixture was irradiated under nitrogen atmosphere for 48h using blue LED (455 $( \pm 15) \mathrm{nm})$. The reaction mixture was then subjected to the work-up procedure as described in the General Procedure 3. Purification was performed using flash chromatography on silica gel using PE/EtOAc solvent mixture as eluent to provide the title compound in $82 \%$ yield $(35.9 \mathrm{mg}) .{ }^{1} \mathrm{H}$ NMR $(400 \mathrm{MHz}$, $\left.\mathrm{CDCl}_{3}\right) \delta 7.69(\mathrm{~d}, J=8.3 \mathrm{~Hz}) \& 7.65(\mathrm{~d}, J=8.3 \mathrm{~Hz}, 1 \mathrm{H}$ combined), $7.53(\mathrm{~s}) \& 7.43(\mathrm{~s}, 1 \mathrm{H}$ combined), $7.31(\mathrm{~d}, J=5.4 \mathrm{~Hz}) \& 7.27(\mathrm{~d}, J=5.4 \mathrm{~Hz}, 1 \mathrm{H}$ combined), $7.16-7.09(\mathrm{~m}, 1 \mathrm{H}), 7.08$ $(\mathrm{dd}, J=8.3,1.3 \mathrm{~Hz}) \& 6.98(\mathrm{dd}, J=8.3,1.3 \mathrm{~Hz}, 1 \mathrm{H}$ combined), $4.52(\mathrm{~s}) \& 4.46(\mathrm{~s}, 2 \mathrm{H}$ combined), 2.80 (s) \& 2.75 (s, 3H combined), 2.02 (s) \& 2.00 (s, 3H combined). ${ }^{13} \mathrm{C}$ NMR (101 $\left.\mathrm{MHz}, \mathrm{CDCl}_{3}\right) \delta 171.1 \& 170.8,140.1 \& 139.9,139.0 \& 138.8,133.7 \& 132.8,127.5 \& 127.0$, $124.6,123.7 \& 123.7,123.1 \& 123.1,122.8 \& 122.7,121.0,54.3 \& 50.6,35.5 \& 33.8,21.9 \&$ 21.5. HRMS (APCI): $\mathrm{m} / z$ calculated. for $\mathrm{C}_{12} \mathrm{H}_{13} \mathrm{NOS}[\mathrm{M}+\mathrm{H}]^{+}: 220.0791$; found 212.0793. 


\section{$N$-((6-acetylpyridin-2-yl)methyl)- $N$-methylacetamide (33)}<smiles>CC(=O)c1cccc(CN(C)C(C)=O)n1</smiles>

The compound was prepared according to the General Procedure 3 using 1-(6-bromopyridin-2-yl)ethan-1-one (40.0 mg, $0.2 \mathrm{mmol})$, mpg-CN (10.0 mg), $\mathrm{NiBr}_{2} \bullet$ glyme (3.2 mg, 5 mol\%), 2,2'-bipyridine (1.6 mg $5 \mathrm{~mol} \%), N, N$-dimethylacetamide $(1.0 \mathrm{~mL})$ and 2,6-lutidine (70 $\mu \mathrm{L} 0.6 \mathrm{mmol})$. The reaction mixture was irradiated under nitrogen atmosphere for 48h using blue LED $(455( \pm 15)$ $\mathrm{nm})$. The reaction mixture was then subjected to the work-up procedure as described in the General Procedure 3. Purification was performed using flash chromatography on silica gel using $\mathrm{DCM} / \mathrm{MeOH}$ solvent mixture as eluent to provide the title compound in 52\% yield (21.4 mg). ${ }^{1} \mathrm{H}$ NMR (400 MHz, $\left.\mathrm{CDCl}_{3}\right) \delta 7.97$ (d, $\left.J=7.9 \mathrm{~Hz}\right) \& 7.92$ (d, $J=7.9 \mathrm{~Hz}, 1 \mathrm{H}$ combined), $7.85\left(\mathrm{dd}, J_{1}=J_{2}=7.7 \mathrm{~Hz}\right) \& 7.78\left(\mathrm{dd}, J_{1}=J_{2}=7.7 \mathrm{~Hz}, 1 \mathrm{H}\right.$ combined $), 7.44(\mathrm{~d}, J=7.6 \mathrm{~Hz}) \&$ 7.34 (d, $J=7.6 \mathrm{~Hz}, 1 \mathrm{H}$ combined), 4.75 (s) \& 4.67 (s, 2H combined), 3.14 (s) \& 3.01 (s, 3H combined), 2.71 (s) \& 2.70 ( $\mathrm{s}, 3 \mathrm{H}$ combined), 2.21 (s) \& 2.19 (s, 3H combined). ${ }^{13} \mathrm{C}$ NMR (101 $\left.\mathrm{MHz} \mathrm{CDCl}_{3}\right) \delta 200.3 \& 200.0,171.5 \& 171.1,157.0 \& 156.2,153.8 \& 153.1,138.0 \& 137.7$, $125.7 \& 124.2,120.5 \& 120.2,56.0 \& 52.8,36.9 \& 34.325 .8(x 2), 21.8 \& 21.6$. EI-MS: $\mathrm{m} / \mathrm{z}$ calculated. for $\mathrm{C}_{11} \mathrm{H}_{14} \mathrm{~N}_{2} \mathrm{O}_{2}[\mathrm{M}]^{+}:$206.10498; found 206.10478.

\section{$N$-methyl- $N$-(quinolin-2-ylmethyl)acetamide (34)}<smiles>CC(=O)N(C)Cc1ccc2ccccc2n1</smiles>

The compound was prepared according to the General Procedure 3 using 2-chloroquinoline (32.6 mg, $0.2 \mathrm{mmol})$, mpg-CN (10.0 mg), $\mathrm{NiCl}_{2} \bullet$ glyme (2.2 mg, $5 \mathrm{~mol} \%$ ), 2,2'-bipyridine (1.6 mg $5 \mathrm{~mol} \%$ ), $N, N$ dimethylacetamide $(1.0 \mathrm{~mL})$ and 2,6-lutidine $(70 \mu \mathrm{L} 0.6 \mathrm{mmol})$. The reaction mixture was irradiated under nitrogen atmosphere for 48h using blue LED (455 ( \pm 15$) \mathrm{nm})$. The reaction mixture was then subjected to the work-up procedure as described in the General Procedure 3. Purification was performed using flash chromatography on silica gel using PE/EtOAc solvent mixture as eluent to provide the title compound in $83 \%$ yield $(35.5 \mathrm{mg}) .{ }^{1} \mathrm{H} \mathrm{NMR}(400 \mathrm{MHz}$, $\left.\mathrm{CDCl}_{3}\right) \delta 8.17(\mathrm{~d}, J=8.5 \mathrm{~Hz}) \& 8.10(\mathrm{~d}, J=8.5 \mathrm{~Hz}, 1 \mathrm{H}$ combined $), 8.04(\mathrm{~d}, J=8.5 \mathrm{~Hz}) \& 8.03$ (d, $J=8.5 \mathrm{~Hz}, 1 \mathrm{H}$ combined), $7.84-7.64(\mathrm{~m}, 2 \mathrm{H}), 7.56-7.46(\mathrm{~m}, 1 \mathrm{H}), 7.38(\mathrm{~d}, J=8.5 \mathrm{~Hz}) \&$ 7.27 (d, $J=8.5 \mathrm{~Hz}, 1 \mathrm{H}$ combined), 4.88 (s) \& 4.78 (s, 2H combined), 3.04 (s) \& 3.03 (s, 3H combined), 2.18 (s) \& 2.17 (s, $3 \mathrm{H}$ combined). ${ }^{13} \mathrm{C} \mathrm{NMR}\left(101 \mathrm{MHz}, \mathrm{CDCl}_{3}\right) \delta 171.5 \& 171.1$, $158.0 \& 157.1,148.0 \& 147.5,137.5 \& 137.1,130.1 \& 129.7,129.1 \& 129.0,127.7 \& 127.6$, $127.5 \& 127.4,126.7 \& 126.4,120.2 \& 118.1,56.9 \& 53.3,36.3 \& 34.4,21.8 \&$ 21.7. EI-MS: $\mathrm{m} / \mathrm{z}$ calculated. for $\mathrm{C}_{13} \mathrm{H}_{14} \mathrm{~N}_{2} \mathrm{O}[\mathrm{M}]^{+}:$214.11006; found 214.11012 . 


\section{$N$-methyl- $N$-(quinolin-5-ylmethyl)acetamide (35)}<smiles>CC(=O)N(C)Cc1cccc2ncccc12</smiles>

The compound was prepared according to the General Procedure 3 using 5-bromoquinoline (41.2 mg, $0.2 \mathrm{mmol}), \operatorname{mpg}-\mathrm{CN}(10.0 \mathrm{mg})$, $\mathrm{NiBr}_{2} \bullet$ glyme (3.2 mg, $5 \mathrm{~mol} \%$ ), 2,2'-bipyridine (1.6 mg $5 \mathrm{~mol} \%$ ), $N, N$ dimethylacetamide $(1.0 \mathrm{~mL})$ and 2,6-lutidine $(70 \mu \mathrm{L} 0.6 \mathrm{mmol})$. The reaction mixture was irradiated under nitrogen atmosphere for 48h using blue LED $(455( \pm 15)$ $\mathrm{nm})$. The reaction mixture was then subjected to the work-up procedure as described in the General Procedure 3. Purification was performed using flash chromatography on silica gel using $\mathrm{PE} / \mathrm{EtOAc}$ solvent mixture as eluent to provide the title compound in $61 \%$ yield $(26.1$ mg). ${ }^{1} \mathrm{H}$ NMR (400 MHz, $\left.\mathrm{CDCl}_{3}\right) \delta 8.20(\mathrm{~d}, J=8.5 \mathrm{~Hz}) \& 8.14(\mathrm{~d}, J=8.5 \mathrm{~Hz}, 1 \mathrm{H}$ combined), $8.10-8.02(\mathrm{~m}, 1 \mathrm{H}), 7.85-7.79(\mathrm{~m}, 1 \mathrm{H}), 7.77-7.68(\mathrm{~m}, 1 \mathrm{H}), 7.58-7.50(\mathrm{~m}, 1 \mathrm{H}), 7.42(\mathrm{~d}, J$ $=8.5 \mathrm{~Hz}) \& 7.29(\mathrm{~d}, J=8.5 \mathrm{~Hz}, 1 \mathrm{H}$ combined $), 4.91(\mathrm{~s}) \& 4.80(\mathrm{~s}, 2 \mathrm{H}$ combined), 3.07 (s) \& 3.05 (s, 3H combined), 2.20 (s) \& 2.19 (s, 3H combined). $\left.{ }^{13} \mathrm{C} \mathrm{NMR} \mathrm{(101} \mathrm{MHz,} \mathrm{CDCl}_{3}\right) \delta 171.5$ $\& 171.0,157.9 \& 157.1,147.9 \& 147.8,137.5 \& 137.4,130.1 \& 129.9,129.0 \& 128.6,127.7$ $\& 127.6,127.4 \& 127.3,126.7 \& 126.6,120.3 \& 118.1,56.9 \& 53.0,36.3 \& 34.4,21.8 \& 21.6$. EI-MS: $m / z$ calculated. for $\mathrm{C}_{13} \mathrm{H}_{14} \mathrm{~N}_{2} \mathrm{O}[\mathrm{M}]^{+}: 214.11006$; found 214.11069.

\section{$N$-(isoquinolin-1-ylmethyl)- $N$-methylacetamide (36)}<smiles>CC(=O)N(C)Cc1nccc2ccccc12</smiles>

The compound was prepared according to the General Procedure 3 using 1chloroisoquinoline (32.6 mg, $0.2 \mathrm{mmol}), \mathrm{mpg}-\mathrm{CN}$ (10.0 mg), NiCl $\mathrm{N}_{2}$ glyme (2.2 mg, $5 \mathrm{~mol} \%$ ), 2,2'-bipyridine (1.6 mg $5 \mathrm{~mol} \%$ ), $N, N$-dimethylacetamide (1.0 mL) and 2,6-lutidine $(70 \mu \mathrm{L} 0.6 \mathrm{mmol})$. The reaction mixture was irradiated under nitrogen atmosphere for $48 \mathrm{~h}$ using blue LED $(455( \pm 15) \mathrm{nm})$. The reaction mixture was then subjected to the work-up procedure as described in the General Procedure 3. Purification was performed using flash chromatography on silica gel using PE/EtOAc solvent mixture as eluent to provide the title compound in $86 \%$ yield $(36.8 \mathrm{mg}) .{ }^{1} \mathrm{H} \mathrm{NMR}\left(400 \mathrm{MHz}, \mathrm{CDCl}_{3}\right) \delta 8.48(\mathrm{~d}, J$ $=5.7 \mathrm{~Hz}) \& 8.44(\mathrm{~d}, J=5.7 \mathrm{~Hz}, 1 \mathrm{H}$ combined $), 8.35(\mathrm{~d}, J=8.5 \mathrm{~Hz}) \& 8.02(\mathrm{~d}, J=8.5 \mathrm{~Hz}, 1 \mathrm{H}$ combined), $7.87(\mathrm{~d}, J=8.1 \mathrm{~Hz}) \& 7.82(\mathrm{~d}, J=8.1 \mathrm{~Hz}, 1 \mathrm{H}$ combined) $7.73-7.55(\mathrm{~m}, 3 \mathrm{H}), 5.23$ (s) \& $5.11(\mathrm{~s}, 2 \mathrm{H}$ combined), $3.00(\mathrm{~s}) \& 2.96(\mathrm{~s}, 3 \mathrm{H}$ combined), 2.15 (s) \& 2.14 (s, 3H combined). ${ }^{13} \mathrm{C} \mathrm{NMR}\left(101 \mathrm{MHz}, \mathrm{CDCl}_{3}\right) \delta 172.2 \& 170.6,156.6 \& 154.4,142.2 \& 141.2,136.5$ $\& 136.2,130.5 \& 130.2,127.9 \& 127.8,127.7 \& 127.2,126.9 \& 126.2,125.6 \& 123.2,121.0$ $\& 120.5,53.4 \& 50.3,35.6 \& 34.6,21.9 \& 21.7$. EI-MS: $m / z$ calculated. for $\mathrm{C}_{13} \mathrm{H}_{14} \mathrm{~N}_{2} \mathrm{O}[\mathrm{M}]^{+}$: 214.11006; found 214.11034. 


\section{$N$-methyl- $N$-(pyrimidin-5-ylmethyl)acetamide (37)}

${ }_{\mathrm{N}}$ The compound was prepared according to the General Procedure 3 using 5-bromopyrimidine (31.6 $\mathrm{mg}, 0.2 \mathrm{mmol}$ ), mpg-CN (10.0 mg), $\mathrm{NiBr}_{2} \bullet$ glyme (3.2 mg, $5 \mathrm{~mol} \%$ ), 2,2'-bipyridine (1.6 mg $5 \mathrm{~mol} \%$ ), $N, N$-dimethylacetamide (1.0 $\mathrm{mL}$ ) and 2,6-lutidine (70 $\mu \mathrm{L} 0.6 \mathrm{mmol})$. The reaction mixture was irradiated under nitrogen atmosphere for $48 \mathrm{~h}$ using blue LED $(455( \pm 15) \mathrm{nm})$. The reaction mixture was then subjected to the work-up procedure as described in the General Procedure 3. Purification was performed using flash chromatography on silica gel using $\mathrm{DCM} / \mathrm{MeOH}$ solvent mixture as eluent to provide the title compound in $74 \%$ yield $(24.4 \mathrm{mg}) .{ }^{1} \mathrm{H} \mathrm{NMR}\left(400 \mathrm{MHz}, \mathrm{CDCl}_{3}\right) \delta 9.17$ (s) \& $9.12(\mathrm{~s}, 1 \mathrm{H}$ combined), $8.65(\mathrm{~s}) \& 8.60(\mathrm{~s}, 2 \mathrm{H}$ combined), $4.63(\mathrm{~s}) \& 4.54$ (s, 2H combined), $2.99(\mathrm{~s}) \& 2.93$ (s, 3H combined), 2.16 (s) \& 2.13 (s, 3H combined). ${ }^{13} \mathrm{C}$ NMR $(101 \mathrm{MHz}$, $\left.\mathrm{CDCl}_{3}\right) \delta 171.1 \& 170.6,158.5 \& 158.1,156.7 \& 155.5,131.0 \& 130.2,49.9 \& 46.5,36.0 \&$ 33.7, 21.7 \& 21.5. EI-MS: $m / z$ calculated. for $\mathrm{C}_{8} \mathrm{H}_{11} \mathrm{~N}_{3} \mathrm{O}[\mathrm{M}]^{+}:$165.08966; found 165.08986.

\section{tert-Butyl 5-(( $N$-methylacetamido)methyl)-1H-indole-1-carboxylate (38)}

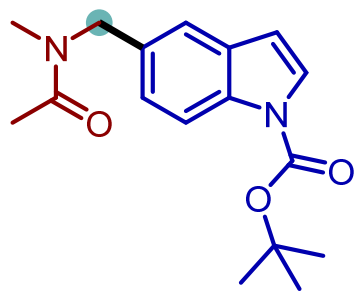

The compound was prepared according to the General Procedure 3 using tert-butyl 5-bromo-1H-indole-1-carboxylate $(59.2$ $\mathrm{mg}, 0.2 \mathrm{mmol}$ ), mpg-CN (10.0 mg), NiBr $2 \bullet g l y m e ~(3.2 \mathrm{mg}, 5 \mathrm{~mol} \%)$, 2,2'-bipyridine (1.6 mg $5 \mathrm{~mol} \%), N, N$-dimethylacetamide $(1.0 \mathrm{~mL})$ and 2,6-lutidine $(70 \mu \mathrm{L} 0.6 \mathrm{mmol})$. The reaction mixture was irradiated under nitrogen atmosphere for $48 \mathrm{~h}$ using blue LED $(455( \pm 15) \mathrm{nm})$. The reaction mixture was then subjected to the work-up procedure as described in the General Procedure 3. Purification was performed using flash chromatography on silica gel using PE/EtOAc solvent mixture as eluent to provide the title compound in $74 \%$ yield $(24.4 \mathrm{mg}) .{ }^{1} \mathrm{H}$ NMR $\left(400 \mathrm{MHz}, \mathrm{CDCl}_{3}\right) \delta$ $8.10(\mathrm{~d}, J=8.0 \mathrm{~Hz}, 1 \mathrm{H}), 7.64(\mathrm{~d}, J=3.7 \mathrm{~Hz}) \& 7.57$ (d, $J=3.6 \mathrm{~Hz}, 1 \mathrm{H}$ combined), $7.33-7.19$ $(\mathrm{m}, 1 \mathrm{H}), 7.07(\mathrm{~d}, J=7.3 \mathrm{~Hz}) \& 6.99(\mathrm{~d}, J=7.4 \mathrm{~Hz}, 1 \mathrm{H}$ combined $), 6.71(\mathrm{~d}, J=3.7 \mathrm{~Hz}) \& 6.53$ (d, $J=3.7 \mathrm{~Hz}, 1 \mathrm{H}$ combined), 4.83 (s) \& 4.75 (s, $2 \mathrm{H}$ combined), 2.96 (s) \& 2.83 (s, 3H combined), 2.15 (s) \& 2.13 (s, 3H combined), 1.66 (s) \& 1.65 (s, 9H combined). ${ }^{13} \mathrm{C} \mathrm{NMR} \mathrm{(101}$ $\left.\mathrm{MHz}, \mathrm{CDCl}_{3}\right) \delta 171.3 \& 170.5,149.7 \& 149.6,135.5 \& 135.4,129.7 \& 129.3,128.4 \& 128.3$, $126.4 \& 126.0,124.6 \& 124.1,123.1 \& 119.6,114.7 \& 114.6,105.8 \& 104.2,84.1 \& 83.8,52.1$ $\& 48.1,35.0 \& 33.9,28.2,21.9 \& 21.5$. HRMS (ESI): $m / z$ calculated. for $\mathrm{C}_{17} \mathrm{H}_{22} \mathrm{~N}_{2} \mathrm{O}_{3}[\mathrm{M}+\mathrm{H}]^{+}$: 303.1703; found 303.1707. 


\section{$N$-(benzo[d]thiazol-2-ylmethyl)- $N$-methylacetamide (39)}

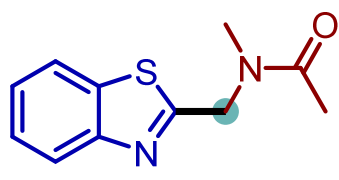

The compound was prepared according to the General Procedure 3 using 2-bromobenzo[d]thiazole (42.8 $\mathrm{mg}, 0.2 \mathrm{mmol}$ ), tetrabutylammonium chloride (66.7 $\mathrm{mg}, 0.24 \mathrm{mmol})$, mpg-CN (10.0 $\mathrm{mg}$ ), $\mathrm{NiCl}_{2} \bullet$ glyme (2.2 mg, $5 \mathrm{~mol} \%$ ), 2,2'-bipyridine (1.6 mg $5 \mathrm{~mol} \%$ ), $N, N$-dimethylacetamide $(1.0 \mathrm{~mL})$ and 2,6-lutidine $(70 \mu \mathrm{L} 0.6 \mathrm{mmol})$. The reaction mixture was irradiated under nitrogen atmosphere for $72 \mathrm{~h}$ using blue LED $(455( \pm 15) \mathrm{nm})$. The reaction mixture was then subjected to the work-up procedure as described in the General Procedure 3. Purification was performed using flash chromatography on silica gel using PE/EtOAc solvent mixture as eluent to provide the title compound in $66 \%$ yield $(29.1 \mathrm{mg}) .{ }^{1} \mathrm{H}$ NMR $\left(400 \mathrm{MHz}, \mathrm{CDCl}_{3}\right) \delta 7.99(\mathrm{~d}, J=7.9 \mathrm{~Hz})$ \& $7.98(\mathrm{~d}, J=7.9 \mathrm{~Hz}, 1 \mathrm{H}$ combined), $7.87(\mathrm{~d}, J=7.8 \mathrm{~Hz}) \& 7.84(\mathrm{~d}, J=7.8 \mathrm{~Hz}, 1 \mathrm{H}$ combined), $7.53-7.32(\mathrm{~m}, 2 \mathrm{H}), 4.96(\mathrm{~s}) \& 4.87$ (s, $1 \mathrm{H}$ combined), 3.11 (s) \& 3.08 (s, 3H combined), 2.22 (s) \& 2.17 (s, 3H combined). ${ }^{13} \mathrm{C} \mathrm{NMR}\left(101 \mathrm{MHz}, \mathrm{CDCl}_{3}\right) \delta 171.1 \& 171.0,168.2 \& 167.9$, $153.3 \& 152.7,135.7 \& 134.9,126.5 \& 126.1,125.6 \& 125.3,123.2 \& 123.0,121.9 \& 121.8$, $53.2 \& 49.4,36.5 \& 34.5,21.7 \&$ 21.6. EI-MS: $\mathrm{m} / \mathrm{z}$ calculated. for $\mathrm{C}_{11} \mathrm{H}_{12} \mathrm{~N}_{2} \mathrm{OS}[\mathrm{M}]^{+}$: 220.06649 ; found 220.06674 .

\section{$N$-methyl- $N$-(thieno[2,3-d]pyrimidin-4-ylmethyl)acetamide (40)}<smiles>CC(=O)N(C)Cc1ncnc2sccc12</smiles>

The compound was prepared according to the General Procedure 3 using 4-bromothieno[2,3-d]pyrimidine (43.0 mg, $0.2 \mathrm{mmol}), \mathrm{mpg}-\mathrm{CN}(10.0$ $\mathrm{mg}$ ), $\mathrm{NiCl}_{2} \bullet$ glyme (2.2 mg, $5 \mathrm{~mol} \%$ ), 2,2'-bipyridine (1.6 mg $5 \mathrm{~mol} \%$ ), $N, N$ dimethylacetamide $(1.0 \mathrm{~mL})$ and 2,6-lutidine $(70 \mu \mathrm{L} 0.6 \mathrm{mmol})$. The reaction mixture was irradiated under nitrogen atmosphere for 48h using blue LED $(455( \pm 15) \mathrm{nm})$. The reaction mixture was then subjected to the work-up procedure as described in the General Procedure 3. Purification was performed using flash chromatography on silica gel using $\mathrm{PE} /$ EtOAc solvent mixture as eluent to provide the title compound in $81 \%$ yield $(35.8 \mathrm{mg}) .{ }^{1} \mathrm{H}$ NMR (400 MHz, $\left.\mathrm{CDCl}_{3}\right) \delta 9.04(\mathrm{~s}) \& 8.99(\mathrm{~s}, 1 \mathrm{H}$ combined), $7.62(\mathrm{~d}, J=6.1 \mathrm{~Hz}) \& 7.58(\mathrm{~d}, J$ $=6.1 \mathrm{~Hz}, 1 \mathrm{H}$ combined), $7.52(\mathrm{~d}, J=6.1 \mathrm{~Hz}) \& 7.35(\mathrm{~d}, J=6.1 \mathrm{~Hz}, 1 \mathrm{H}$ combined $), 4.99$ (s) \& 4.92 (s, 2H combined), 3.08 (s) \& 2.99 (s, 3H combined), 2.16 (s) \& 2.15 (s, 3H combined). ${ }^{13} \mathrm{C} \mathrm{NMR}\left(101 \mathrm{MHz}, \mathrm{CDCl}_{3}\right) \delta 171.6 \& 170.9,169.4 \& 169.2,159.8 \& 159.2,153.4 \& 152.9$, $129.0 \& 128.5,127.9 \& 127.5,120.1 \& 118.0,54.2 \& 50.5,36.5 \& 34.6,21.7$. EI-MS: $m / z$ calculated. for $\mathrm{C}_{10} \mathrm{H}_{11} \mathrm{~N}_{3} \mathrm{OS}[\mathrm{M}]^{+}: 221.06173$; found 221.06156 . 
<smiles>CC(=O)N(C)Cc1ccc(C(N)=O)cc1</smiles>

The compound was prepared according to the General Procedure 1 using 4-bromobenzamide (40.0 mg, $0.2 \mathrm{mmol}$ ), mpg-CN (10.0 mg), NiBr2•glyme (3.2 mg, 5 mol\%), 2,2'-bipyridine (1.6 mg $5 \mathrm{~mol} \%), N, N$-dimethylacetamide $(1.0 \mathrm{~mL})$ and 2,6-lutidine (70 $\mu \mathrm{L} 0.6 \mathrm{mmol})$. The reaction mixture was irradiated under nitrogen atmosphere for $48 \mathrm{~h}$ using blue LED $(455( \pm 15) \mathrm{nm})$. The reaction mixture was then subjected to the work-up procedure as described in the General Procedure 1. Purification was performed using flash chromatography on silica gel using PE/EtOAc solvent mixture as eluent to provide the title compound in $85 \%$ yield $\left(35.0 \mathrm{mg}\right.$ ). ${ }^{1} \mathrm{H}$ NMR (400 MHz, DMSO-d6) $\delta 8.00$ (s) \& $7.97(\mathrm{~s}, 1 \mathrm{H}$ combined), $7.90(\mathrm{~d}, J=8.2 \mathrm{~Hz}) \& 7.85(\mathrm{~d}, J=8.2 \mathrm{~Hz}, 2 \mathrm{H}$ combined), 7.39 (s) \& 7.36 (s, $1 \mathrm{H}$ combined), 7.27 (d, J=8.2 Hz, 2H), 4.59 (s) \& 4.52 (s, 2H combined), 2.91 (s) \& 2.79 (s, 3H combined), 2.07 (s) \& 2.03 (s, 3H combined). ${ }^{13} \mathrm{C}$ NMR (101 MHz, DMSO-d 6 ) $\delta 170.2 \& 170.1$, $167.8 \& 167.7,141.3 \& 140.9,133.3 \& 133.0,128.0 \& 127.7,127.2 \& 126.4,53.1 \& 49.5,35.6$ \& 33.2, $21.5 \&$ 21.3. HRMS (ESI): $m / z$ calculated. for $\mathrm{C}_{11} \mathrm{H}_{15} \mathrm{~N}_{2} \mathrm{O}_{2}[\mathrm{M}+\mathrm{H}]^{+}:$207.1128; found 207.1130 .

\section{$N$-methyl- $N$-(4-sulfamoylbenzyl)acetamide (42)}

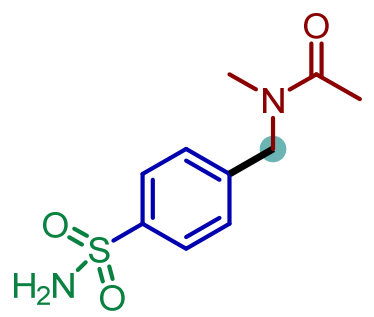

The compound was prepared according to the General Procedure 1 using 4-bromobenzenesulfonamide (47.2 $\mathrm{mg}, 0.2 \mathrm{mmol}$ ), mpg-CN (10.0 mg), NiBr2•glyme (3.2 mg, 5 mol\%), 2,2'-bipyridine (1.6 mg $5 \mathrm{~mol} \%), N, N$-dimethylacetamide $(1.0 \mathrm{~mL})$ and 2,6-lutidine (70 $\mu \mathrm{L} 0.6 \mathrm{mmol}$ ). The reaction mixture was irradiated under nitrogen atmosphere for $48 \mathrm{~h}$ using blue LED $(455( \pm 15) \mathrm{nm})$. The reaction mixture was then subjected to the work-up procedure as described in the General Procedure 1. Purification was performed using flash chromatography on silica gel using PE/EtOAc solvent mixture as eluent to provide the title compound in $87 \%$ yield $\left(42.2 \mathrm{mg}\right.$ ). ${ }^{1} \mathrm{H}$ NMR (400 MHz, DMSO- $\left.d_{6}\right) \delta 7.83(\mathrm{~d}, J=8.3$ $\mathrm{Hz}) \& 7.78(\mathrm{~d}, J=8.3 \mathrm{~Hz}, 2 \mathrm{H}$ combined), 7.39 (d, $J=8.3 \mathrm{~Hz}, 2 \mathrm{H}), 7.35$ (s) \& 7.33 (s, $1 \mathrm{H}$ combined), 4.64 (s) \& 4.55 (s, 2H combined), 2.93 (s) \& 2.80 (s, 3H combined), 2.08 (s) \& 2.03 (s, 3H combined). ${ }^{13} \mathrm{C}$ NMR (101 MHz, DMSO-d6) $\delta 170.2 \& 170.0,143.1 \& 142.8,142.1 \&$ $141.7,127.8 \& 127.0,126.1 \& 125.8,52.9 \& 49.4,35.7 \& 33.2,21.5 \& 21.2$. HRMS (ESI): $m / z$ calculated. for $\mathrm{C}_{10} \mathrm{H}_{15} \mathrm{~N}_{2} \mathrm{O}_{3} \mathrm{~S}[\mathrm{M}+\mathrm{H}]^{+}$: 243.0798; found 243.0806 . 
<smiles>CC(=O)N(C)Cc1ccc(C(=O)O)cc1</smiles>

The compound was prepared according to the General Procedure 1 using 4-bromobenzoic acid (40.2 mg, $0.2 \mathrm{mmol}), \mathrm{mpg}-\mathrm{CN}(10.0 \mathrm{mg})$, $\mathrm{NiBr}_{2} \bullet$ glyme (3.2 mg, $5 \mathrm{~mol} \%$ ), 2,2'-bipyridine (1.6 mg $5 \mathrm{~mol} \%$ ), $N, N$ dimethylacetamide $(1.0 \mathrm{~mL})$ and 2,6-lutidine $(70 \mu \mathrm{L} 0.6 \mathrm{mmol})$. The reaction mixture was irradiated under nitrogen atmosphere for $48 \mathrm{~h}$ using as described in the General Procedure 1. Purification was performed using flash chromatography on silica gel using PE/EtOAc solvent mixture as eluent to provide the title compound in 68\% yield (28.2 mg). ${ }^{1} \mathrm{H}$ NMR (400 MHz, DMSO-d $) \delta 7.92(\mathrm{~d}, J=8.1 \mathrm{~Hz}) \&$ $7.88(\mathrm{~d}, J=8.1 \mathrm{~Hz}, 2 \mathrm{H}$ combined), $7.24(\mathrm{~d}, J=8.1 \mathrm{~Hz}) \& 7.23(\mathrm{~d}, J=8.1 \mathrm{~Hz}, 2 \mathrm{H}$ combined), 4.60 (s) \& 4.53 (s, $2 \mathrm{H}$ combined), 2.91 (s) \& 2.79 (s, 3H combined), 2.07 (s) \& 2.03 (s, 3H combined). ${ }^{13} \mathrm{C}$ NMR (101 MHz, DMSO-d 6 ) $\delta 170.2 \& 170.1,167.9,142.3 \& 141.8,131.2 \&$ 131.0, $129.8 \& 129.5,127.3 \& 126.4,53.2 \& 49.6,35.7 \& 33.3,21.6 \& 21.3$. HRMS (ESI): $\mathrm{m} / \mathrm{z}$ calculated. for $\mathrm{C}_{11} \mathrm{H}_{14} \mathrm{NO}_{3}[\mathrm{M}+\mathrm{H}]^{+}:$208.0968; found 208.0973.

\section{$N$-(4-hydroxybenzyl)-N-methylacetamide (44)}<smiles>CC(=O)N(C)Cc1ccc(O)cc1</smiles>

The compound was prepared according to the General Procedure 1 using 4-bromophenol (34.6 mg, $0.2 \mathrm{mmol})$, mpg-CN (10.0 mg), $\mathrm{NiBr}_{2} \bullet$ glyme (3.2 mg, $5 \mathrm{~mol} \%$ ), 2,2'-bipyridine (1.6 mg $5 \mathrm{~mol} \%$ ), $N, N$ dimethylacetamide $(1.0 \mathrm{~mL})$ and 2,6-lutidine $(70 \mu \mathrm{L} 0.6 \mathrm{mmol})$. The reaction mixture was irradiated under nitrogen atmosphere for 48h using blue LED (455 $( \pm 15) \mathrm{nm})$. The reaction mixture was then subjected to the work-up procedure as described in the General Procedure 1. Purification was performed using flash chromatography on silica gel using PE/EtOAc solvent mixture as eluent to provide the title compound in $63 \%$ yield $(22.5 \mathrm{mg}) .{ }^{1} \mathrm{H}$ NMR $(400 \mathrm{MHz}$, $\left.\mathrm{CDCl}_{3}\right) \delta 8.67(\mathrm{~s}, 1 \mathrm{H}), 7.07(\mathrm{~d}, J=8.5 \mathrm{~Hz}) \& 6.97(\mathrm{~d}, J=8.5 \mathrm{~Hz}, 2 \mathrm{H}$ combined $), 6.87(\mathrm{~d}, J=$ $8.5 \mathrm{~Hz}) \& 6.82(\mathrm{~d}, J=8.5 \mathrm{~Hz}, 2 \mathrm{H}$ combined), $4.50(\mathrm{~s}) \& 4.42(\mathrm{~s}, 2 \mathrm{H}$ combined), $2.92(\mathrm{~s}, 1 \mathrm{H}) \&$ $2.91\left(\mathrm{~s}, 3 \mathrm{H}\right.$ combined), 2.18 (s) \& 2.14 (s, 3H combined). ${ }^{13} \mathrm{C} \mathrm{NMR}\left(101 \mathrm{MHz}, \mathrm{CDCl}_{3}\right) \delta 171.9$ $\& 171.5,156.7 \& 156.4,129.5 \& 127.8,127.9 \& 126.8,116.1 \& 115.7,54.06 \& 50.4,35.5 \&$ 33.8, 21.7 \& 21.4. EI-MS: $m / z$ calculated. for $\mathrm{C}_{10} \mathrm{H}_{13} \mathrm{NO}_{2}[\mathrm{M}]^{+}:$179.09408; found 179.09440. 
<smiles>CC(=O)N(C)Cc1ccc(B(O)O)cc1</smiles>

The compound was prepared according to the General Procedure 1 using (4-bromophenyl)boronic acid (40.0 mg, 0.2 mmol), mpg-CN (10.0 mg), NiBr $2 \bullet$ glyme (3.2 mg, 5 mol\%), 2,2'bipyridine (1.6 mg $5 \mathrm{~mol} \%), N, N$-dimethylacetamide $(1.0 \mathrm{~mL})$ and 2,6-lutidine ( $70 \mu \mathrm{L} 0.6 \mathrm{mmol}$ ). The reaction mixture was irradiated under nitrogen atmosphere for $48 \mathrm{~h}$ using blue LED $(455( \pm 15) \mathrm{nm})$. The reaction mixture was then subjected to the workup procedure as described in the General Procedure 1. Purification was performed using flash chromatography on silica gel using PE/EtOAc solvent mixture as eluent to provide the title compound in $82 \%$ yield $(33.9 \mathrm{mg}) .{ }^{1} \mathrm{H}$ NMR (400 MHz, DMSO-d $) \delta 7.98$ (s) \& $7.94(\mathrm{~s}, 2 \mathrm{H}$ combined), 7.78 (d, $J=7.9 \mathrm{~Hz}) \& 7.73(\mathrm{~d}, J=7.9 \mathrm{~Hz}, 2 \mathrm{H}$ combined), 7.14 (d, $J=7.9 \mathrm{~Hz}, 2 \mathrm{H})$, 4.54 (s) \& 4.47 (s, 2H combined), 2.88 (s) \& 2.77 (s, 3H combined), 2.05 (s) \& 2.01 (s, 3H combined). ${ }^{13} \mathrm{C}$ NMR (101 MHz, DMSO- $\left.d_{6}\right) \delta 170.0,139.72 \& 139.3,134.6 \& 134.3,126.4 \&$ 125.5, $53.3 \& 49.6,35.5 \& 33.2,21.6 \& 21.3$. HRMS (ESI): $\mathrm{m} / \mathrm{z}$ calculated. for $\mathrm{C}_{10} \mathrm{H}_{15} \mathrm{BNO}_{3}$ $[\mathrm{M}+\mathrm{H}]^{+}:$2081140; found 208.1143.

\section{$N$-(4-acetylbenzyl)- $N$-methylformamide (46)}<smiles>CC(=O)C=Cc1ccc(C(C)=O)cc1</smiles>

The compound was prepared according to the General Procedure 4 using 1-(4-bromophenyl)ethan-1-one (39.8 mg, 0.2 mmol), mpg-CN (10.0 mg), NiBr2•glyme (3.2 mg, 5 mol\%), 2,2'bipyridine $(1.6 \mathrm{mg} 5 \mathrm{~mol} \%), N, N$-dimethylformamide $(1.0 \mathrm{~mL})$ and 2,6-lutidine $(70 \mu \mathrm{L} 0.6$ $\mathrm{mmol})$. The reaction mixture was irradiated under nitrogen atmosphere for $72 \mathrm{~h}$ using blue LED $(455( \pm 15) \mathrm{nm})$. The reaction mixture was then subjected to the work-up procedure as described in the General Procedure 4. Purification was performed using flash chromatography on silica gel using PE/EtOAc solvent mixture as eluent to provide the title compound in 64\% yield (24.4 $\mathrm{mg}$ ). The same compound was synthesized from 1-(4-chlorophenyl)ethan-1-one (30.8 mg, 0.2 mmol), mpg-CN (10.0 mg), $\mathrm{NiCl}_{2} \bullet$ glyme (2.2 mg, 5 mol\%), 2,2'-bipyridine (1.6 mg 5 mol\%), $N$, $N$-dimethylformamide $(1.0 \mathrm{~mL})$ and 2,6-lutidine $(70 \mu \mathrm{L} 0.6 \mathrm{mmol})$ with an isolated yield of $73 \%$ (27.8 mg). ${ }^{1} \mathrm{H}$ NMR (400 MHz, $\left.\mathrm{CDCl}_{3}\right) \delta 8.29$ (s) \& 8.18 (s, 1H combined), 7.95 (d, $J=$ $8.3 \mathrm{~Hz}) \& 7.92(\mathrm{~d}, J=8.3 \mathrm{~Hz}, 2 \mathrm{H}$ combined), $7.33(\mathrm{~d}, J=8.3 \mathrm{~Hz}) \& 7.30(\mathrm{~d}, J=8.3 \mathrm{~Hz}, 2 \mathrm{H}$ combined), 4.57 (s) \& 4.45 (s, $2 \mathrm{H}$ combined), 2.87 (s) \& 2.79 (s, 3H combined), 2.60 (s) \& 2.58 (s, 3H combined). ${ }^{13} \mathrm{C}$ NMR $\left(101 \mathrm{MHz}, \mathrm{CDCl}_{3}\right) \delta 197.7 \& 197.5,162.8 \& 162.7,141.5 \& 141.2$, $137.1 \& 136.6,129.1 \& 128.9,128.3 \& 127.6,53.2 \& 47.6,34.3 \& 29.7,26.7 \& 26.7$. HRMS (APCI): $m / z$ calculated. for $\mathrm{C}_{11} \mathrm{H}_{14} \mathrm{NO}_{2}[\mathrm{M}+\mathrm{H}]^{+}: 192.1019$; found192.1023. 


\section{$N$-(4-acetylbenzyl)- $N$-methylpropionamide (47)}

MeOc)

The compound was prepared according to the General Procedure 4 using 1-(4-chlorophenyl)ethan-1-one (30.8 mg, 0.2 mmol), mpg-CN (10.0 mg), $\mathrm{NiCl}_{2} \bullet$ glyme (2.2 mg, $5 \mathrm{~mol} \%$ ), 2,2'bipyridine (1.6 mg $5 \mathrm{~mol} \%), N, N$-dimethylpropionamide $(1.0 \mathrm{~mL})$ and 2,6-lutidine $(70 \mu \mathrm{L} 0.6$ mmol). The reaction mixture was irradiated under nitrogen atmosphere for $72 \mathrm{~h}$ using blue LED $(455( \pm 15) \mathrm{nm})$. The reaction mixture was then subjected to the work-up procedure as described in the General Procedure 4. Purification was performed using flash chromatography on silica gel using PE/EtOAc solvent mixture as eluent to provide the title compound in 84\% yield (36.8 mg). ${ }^{1} \mathrm{H}$ NMR (300 MHz, $\left.\mathrm{CDCl}_{3}\right) \delta 7.93(\mathrm{~d}, J=8.3 \mathrm{~Hz}) \& 7.88(\mathrm{~d}, J=8.3 \mathrm{~Hz}, 2 \mathrm{H}$ combined), $7.29(\mathrm{~d}, J=8.3 \mathrm{~Hz}) \& 7.23(\mathrm{~d}, J=8.3 \mathrm{~Hz}, 2 \mathrm{H}$ combined), 4.62 (s) \& 4.57 (s, 2H combined), $2.94(\mathrm{~s}) \& 2.91$ (s, 3H combined), 2.58 (s) \& 2.56 (s, 3H combined), 2.40 (q, J=7.4 Hz) \& 2.35 (q, $J=7.4 \mathrm{~Hz}, 2 \mathrm{H}$ combined) $1.17(\mathrm{t}, J=7.4 \mathrm{~Hz}) \& 1.13$ (t, $J=7.4 \mathrm{~Hz}, 3 \mathrm{H}$ combined). ${ }^{13} \mathrm{C}$ $\operatorname{NMR}\left(75 \mathrm{MHz}, \mathrm{CDCl}_{3}\right) \delta 197.82 \& 197.6,174.3 \& 174.1,143.2 \& 142.3,136.6 \& 136.2,129.0$ $\& 128.7,128.0 \& 126.4,53.1 \& 50.7,35.0 \& 34.2,26.8 \& 26.7,26.3,9.6 \& 9.3$. HRMS (APCI): $\mathrm{m} / \mathrm{z}$ calculated. for $\mathrm{C}_{13} \mathrm{H}_{18} \mathrm{NO}_{2}[\mathrm{M}+\mathrm{H}]^{+}:$220.1332; found 220.1337 .

\section{$N$-(4-acetylbenzyl)- $N$-methylisobutyramide (48)}<smiles>CC(=O)c1ccc(C=NC(=O)C(C)C)cc1</smiles>

The compound was prepared according to the General Procedure 4 using 1-(4-chlorophenyl)ethan-1-one (30.8 mg, 0.2 mmol), mpg-CN (10.0 mg), $\mathrm{NiCl}_{2} \bullet$ glyme (2.2 mg, $5 \mathrm{~mol} \%$ ), 2,2'bipyridine (1.6 mg $5 \mathrm{~mol} \%$ ), tetrabutylammonium chloride (5.6 mg, $10 \mathrm{~mol} \%$ ), $\mathrm{N}, \mathrm{N}$ dimethylisobutyramide (230.4 mg, $2.0 \mathrm{mmol}), 2,6$-lutidine $(70 \mu \mathrm{L} 0.6 \mathrm{mmol})$ and $\mathrm{CH}_{3} \mathrm{CN}(0.8$ $\mathrm{mL})$. The reaction mixture was irradiated under nitrogen atmosphere for $72 \mathrm{~h}$ using blue LED $(455( \pm 15) \mathrm{nm})$. The reaction mixture was then subjected to the work-up procedure as described in the General Procedure 4. Purification was performed using flash chromatography on silica gel using PE/EtOAc solvent mixture as eluent to provide the title compound in $81 \%$ yield (37.7 mg). ${ }^{1} \mathrm{H}$ NMR $\left(300 \mathrm{MHz}, \mathrm{CDCl}_{3}\right) \delta 7.90(\mathrm{~d}, J=8.2 \mathrm{~Hz}) \& 7.85(\mathrm{~d}, J=8.2 \mathrm{~Hz}, 2 \mathrm{H}$ combined), $7.25(\mathrm{~d}, J=8.2 \mathrm{~Hz}) \& 7.20(\mathrm{~d}, J=8.2 \mathrm{~Hz}, 2 \mathrm{H}$ combined), 2.93 (s) \& 2.90 (s, 2H combined), $2.81(\mathrm{~h}, J=6.7 \mathrm{~Hz}) \& 2.71(\mathrm{~h}, J=6.7 \mathrm{~Hz}, 1 \mathrm{H}$ combined), 2.55 (s) \& 2.53 (s, 3H combined), $1.12(\mathrm{~d}, J=6.7 \mathrm{~Hz}) \& 1.08(\mathrm{~d}, J=6.7 \mathrm{~Hz}, 6 \mathrm{H}$ combined $) .{ }^{13} \mathrm{C} \mathrm{NMR}\left(75 \mathrm{MHz}, \mathrm{CDCl}_{3}\right) \delta 197.8$ $\& 197.5,177.7 \& 177.3,143.3 \& 142.5,136.5 \& 136.2,129.0 \& 128.7,127.9 \& 126.3,53.0 \&$ 50.8, $34.9 \& 34.3,30.4,26.6,19.8 \&$ 19.3. HRMS (APCI): $\mathrm{m} / \mathrm{z}$ calculated. for $\mathrm{C}_{14} \mathrm{H}_{20} \mathrm{NO}_{2}$ $[\mathrm{M}+\mathrm{H}]^{+}:$234.1489; found 234.1493. 
<smiles>CCN(C(C)=O)C(C)=O</smiles>

The compound was prepared according to the General Procedure 4 using 1-(4-chlorophenyl)ethan-1-one (30.8 mg, 0.2 mmol), mpg-CN (10.0 mg), $\mathrm{NiCl}_{2} \bullet$ glyme (2.2 mg, $\left.5 \mathrm{~mol} \%\right)$, 2,2'bipyridine (1.6 mg $5 \mathrm{~mol} \%$ ), tetrabutylammonium chloride (5.6 mg, $10 \mathrm{~mol} \%$ ), $\mathrm{N}, \mathrm{N}$ diethylacetamide (230.4 mg, $2.0 \mathrm{mmol})$, 2,6-lutidine $(70 \mu \mathrm{L} 0.6 \mathrm{mmol})$ and $\mathrm{CH}_{3} \mathrm{CN}(0.8 \mathrm{~mL})$. The reaction mixture was irradiated under nitrogen atmosphere for $72 \mathrm{~h}$ using blue LED (455 $( \pm 15) \mathrm{nm})$. The reaction mixture was then subjected to the work-up procedure as described in the General Procedure 4. Purification was performed using flash chromatography on silica gel using PE/EtOAc solvent mixture as eluent to provide the title compound in 59\% yield (27.5 mg). ${ }^{1} \mathrm{H}$ NMR (400 MHz, $\left.\mathrm{CDCl}_{3}\right) \delta 7.94(\mathrm{~d}, J=8.2 \mathrm{~Hz}), \& 7.91$ (d, $J=8.2 \mathrm{~Hz}, 2 \mathrm{H}$ combined), $7.40(\mathrm{~d}, J=8.2 \mathrm{~Hz}) \& 7.35(\mathrm{~d}, J=8.2 \mathrm{~Hz}, 2 \mathrm{H}$ combined), 6.03 (q, $J=7.0 \mathrm{~Hz}), 5.09$ (q, $J=7.0$ Hz, $1 \mathrm{H}$ combined), $3.42(\mathrm{dq}, J=14.3,7.1 \mathrm{~Hz}) \& 3.18(\mathrm{dq}, J=14.3,7.1 \mathrm{~Hz}, 1 \mathrm{H}$ combined), $3.05(\mathrm{dq}, J=14.3,7.1 \mathrm{~Hz}) \& 2.94(\mathrm{dq}, J=14.3,7.1 \mathrm{~Hz}, 1 \mathrm{H}$ combined), $2.60(\mathrm{~s}) \& 2.59(\mathrm{~s}, 3 \mathrm{H}$ combined), 2.19 (s) \& 2.17 (s, 3H combined), $1.67(\mathrm{~d}, J=6.9 \mathrm{~Hz}) \& 1.55(\mathrm{~d}, J=6.9 \mathrm{~Hz}, 3 \mathrm{H}$ combined), $1.01(\mathrm{dt}, J=14.3,7.1 \mathrm{~Hz}, 3 \mathrm{H}) .{ }^{13} \mathrm{C} \mathrm{NMR}\left(101 \mathrm{MHz}, \mathrm{CDCl}_{3}\right) \delta 197.8 \& 197.6,170.8$ $\& 170.8,147.0 \& 146.3,136.3 \& 136.2,128.8 \& 128.6,127.7 \& 126.9,56.3 \& 50.9,39.3 \&$ 38.1, 26.7, $22.4 \& 21.9,18.6 \& 16.9,16.2 \& 14.6$. HRMS (APCI): $\mathrm{m} / \mathrm{z}$ calculated. for $\mathrm{C}_{14} \mathrm{H}_{20} \mathrm{NO}_{2}[\mathrm{M}+\mathrm{H}]^{+}:$234.1489; found 234.1486.

\section{$N$-(4-cyanobenzyl)- $N$-methylformamide (50)}<smiles>CN(C=O)C=O</smiles>

The compound was prepared according to the General Procedure 4 using 4-bromobenzonitrile (36.4 mg, $0.2 \mathrm{mmol}$ ), mpg-CN (10.0 mg), NiBr $2 \bullet$ glyme (3.2 mg, 5 mol\%), 2,2'-bipyridine (1.6 mg 5 mol\%), $N, N$-dimethylformamide $(1.0 \mathrm{~mL})$ and 2,6-lutidine $(70 \mu \mathrm{L} 0.6 \mathrm{mmol})$. The reaction mixture was irradiated under nitrogen atmosphere for $72 \mathrm{~h}$ using blue LED $(455( \pm 15) \mathrm{nm})$. The reaction mixture was then subjected to the work-up procedure as described in the General Procedure 4. Purification was performed using flash chromatography on silica gel using $\mathrm{PE} /$ EtOAc solvent mixture as eluent to provide the title compound in $62 \%$ yield $(21.5 \mathrm{mg}) .{ }^{1} \mathrm{H}$ NMR (400 MHz, $\left.\mathrm{CDCl}_{3}\right) \delta 8.28(\mathrm{~s}) \& 8.18(\mathrm{~s}, 1 \mathrm{H}$ combined), $7.68(\mathrm{~d}, J=8.3 \mathrm{~Hz}) \& 7.63(\mathrm{~d}, J$ $=8.3 \mathrm{~Hz}, 2 \mathrm{H}$ combined), $7.35(\mathrm{~d}, J=8.3 \mathrm{~Hz}) \& 7.32(\mathrm{~d}, J=8.3 \mathrm{~Hz}, 2 \mathrm{H}$ combined $), 4.56(\mathrm{~s}) \&$ $4.46\left(\mathrm{~s}, 2 \mathrm{H}\right.$ combined), $2.88(\mathrm{~s}) \& 2.79$ (s, 3H combined). ${ }^{13} \mathrm{C} \mathrm{NMR}\left(101 \mathrm{MHz}, \mathrm{CDCl}_{3}\right) \delta 162.8$ $\& 162.8,141.6 \& 141.4,132.9 \& 132.6,128.8 \& 128.0,118.6 \& 118.4,112.3 \& 111.8,53.1 \&$ 
47.7, 34.3 \& 29.8 HRMS (APCI): $m / z$ calculated. for $\mathrm{C}_{10} \mathrm{H}_{11} \mathrm{~N}_{2} \mathrm{O}[\mathrm{M}+\mathrm{H}]^{+}:$175.0866; found 175.0868 .

\section{1-([1,1'-biphenyl]-4-ylmethyl)-1,3,3-trimethylurea (51)}

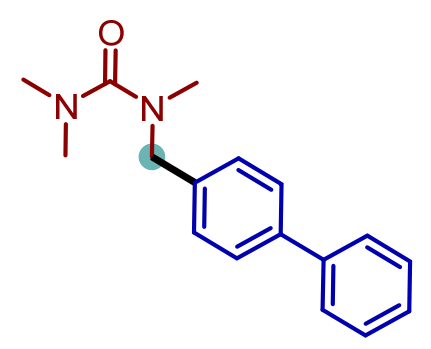

The compound was prepared according to the General Procedure 4 using 4-bromo-1,1'-biphenyl (46.6 mg, $0.2 \mathrm{mmol}$ ), mpg-CN (10.0 mg), NiBr2•glyme (3.2 mg, 5 mol\%), 2,2'-bipyridine (1.6 $\mathrm{mg} 5 \mathrm{~mol} \%), 1,1,3,3$-tetramethylurea $(1.0 \mathrm{~mL})$ and 2,6-lutidine (70 $\mu \mathrm{L} 0.6 \mathrm{mmol})$. The reaction mixture was irradiated under nitrogen atmosphere for $72 \mathrm{~h}$ using blue LED $(455( \pm 15) \mathrm{nm})$. The reaction mixture was then subjected to the work-up procedure as described in the General Procedure 4. Purification was performed using flash chromatography on silica gel using PE/EtOAc solvent mixture as eluent to provide the title compound in $73 \%$ yield $(39.1 \mathrm{mg}) .{ }^{1} \mathrm{H} \mathrm{NMR}\left(400 \mathrm{MHz}, \mathrm{CDCl}_{3}\right) \delta 7.61-$ $7.54(\mathrm{~m}, 4 \mathrm{H}), 7.46-7.41(\mathrm{~m}, 2 \mathrm{H}), 7.37-7.31(\mathrm{~m}, 3 \mathrm{H}), 4.42(\mathrm{~s}, 2 \mathrm{H}), 2.87$ (s, 6H), 2.77 (s, 3H). ${ }^{13} \mathrm{C}$ NMR (101 MHz, $\left.\mathrm{CDCl}_{3}\right) \delta 165.6,140.9,140.1,137.2,128.8,128.1,127.4,127.3,127.1$, 53.9, 38.8, 36.7. HRMS (ESI): $m / z$ calculated. for $\mathrm{C}_{17} \mathrm{H}_{21} \mathrm{~N}_{2} \mathrm{O}[\mathrm{M}+\mathrm{H}]^{+}:$: 269.1648; found 269.1653.

\section{1-methyl-5-(pyridin-2-yl)pyrrolidin-2-one (52)}<smiles>CCCCN1C(=O)CC[C@H]1c1ccccn1</smiles>

The compound was prepared according to the General Procedure 4 using 2-chloropyridine (22.6 mg, $0.2 \mathrm{mmol})$, mpg-CN (10.0 mg), $\mathrm{NiCl}_{2} \bullet$ glyme $(2.2 \mathrm{mg}, 5 \mathrm{~mol} \%), 2,2^{\prime}$-bipyridine (1.6 mg $\left.5 \mathrm{~mol} \%\right)$, 1 methylpyrrolidin-2-one $(1.0 \mathrm{~mL})$ and 2,6-lutidine $(70 \mu \mathrm{L} 0.6 \mathrm{mmol})$. The reaction mixture was irradiated under nitrogen atmosphere for $72 \mathrm{~h}$ using blue LED $(455( \pm 15) \mathrm{nm})$. The reaction mixture was then subjected to the work-up procedure as described in the General Procedure 4. Purification was performed using flash chromatography on silica gel using PE/EtOAc solvent mixture as eluent to provide the title compound in $71 \%$ yield $\left(25.0 \mathrm{mg}, \mathrm{C}_{1}: \mathrm{C}_{2}=8: 1\right) .{ }^{1} \mathrm{H} \mathrm{NMR}$ $\left(300 \mathrm{MHz}, \mathrm{CDCl}_{3}\right) \delta 8.57(\mathrm{ddd}, J=4.9,1.7,0.9 \mathrm{~Hz}, 1 \mathrm{H}), 7.68(\mathrm{td}, J=7.7,1.7 \mathrm{~Hz}, 1 \mathrm{H}), 7.20$ (ddd, $J=7.7,4.9,0.9 \mathrm{~Hz}, 1 \mathrm{H}), 7.12(\mathrm{dt}, J=7.7,0.9 \mathrm{~Hz}, 1 \mathrm{H}), 4.65-4.58(\mathrm{~m}, 1 \mathrm{H}), 2.68(\mathrm{~s}, 3 \mathrm{H})$, $2.57-2.34(\mathrm{~m}, 3 \mathrm{H}), 2.04-1.91(\mathrm{~m}, 1 \mathrm{H}) .{ }^{13} \mathrm{C} \mathrm{NMR}\left(75 \mathrm{MHz}, \mathrm{CDCl}_{3}\right) \delta 175.8,160.4,150.1$, 137.2, 122.9, 120.5, 65.8, 29.9, 28.4, 26.5 HRMS (APCI): $\mathrm{m} / \mathrm{z}$ calculated. for $\mathrm{C}_{10} \mathrm{H}_{13} \mathrm{~N}_{2} \mathrm{O}$ $[\mathrm{M}+\mathrm{H}]^{+}:$177.1022; found 177.1023. 


\section{1-methyl-5-(5-methylpyridin-3-yl)pyrrolidin-2-one (53)}<smiles>Cc1cncc(C2CCC(=O)N2C)c1</smiles>

The compound was prepared according to the General Procedure 4 using 3-chloro-5-methylpyridine (25.4 mg, $0.2 \mathrm{mmol}$ ), mpg-CN (10.0 mg), $\mathrm{NiCl}_{2} \bullet$ glyme (2.2 mg, 5 mol\%), 2,2'-bipyridine (1.6 mg $5 \mathrm{~mol} \%)$, 1-methylpyrrolidin-2-one $(1.0 \mathrm{~mL})$ and 2,6-lutidine (70 $\mu \mathrm{L} 0.6 \mathrm{mmol})$. The reaction mixture was irradiated under nitrogen atmosphere for $72 \mathrm{~h}$ using blue LED $(455( \pm 15)$ $\mathrm{nm})$. The reaction mixture was then subjected to the work-up procedure as described in the General Procedure 4. Purification was performed using flash chromatography on silica gel using $\mathrm{PE} / \mathrm{EtOAc}$ solvent mixture as eluent to provide the title compound in $63 \%$ yield (23.9 mg, $\left.\mathrm{C}_{1}: \mathrm{C}_{2}=2.7: 1\right) .{ }^{1} \mathrm{H} \mathrm{NMR}\left(300 \mathrm{MHz}, \mathrm{CDCl}_{3}\right) \delta 8.40(\mathrm{~s}, 1 \mathrm{H}), 8.28(\mathrm{~s}, 1 \mathrm{H}), 7.29(\mathrm{~s}, 1 \mathrm{H}), 4.54$ $-4.47(\mathrm{~m}, 1 \mathrm{H}), 2.65(\mathrm{~s}, 3 \mathrm{H}), 2.62-2.37(\mathrm{~m}, 3 \mathrm{H}), 2.34(\mathrm{~s}, 3 \mathrm{H}), 1.93-1.79(\mathrm{~m}, 1 \mathrm{H}) .{ }^{13} \mathrm{C} \mathrm{NMR}$ $\left(101 \mathrm{MHz}, \mathrm{CDCl}_{3}\right) \delta 175.4,150.4,145.6,136.2,134.1,131.8,62.1,30.0,28.3,28.2,18.5$. EIMS: $m / z$ calculated. for $\mathrm{C}_{11} \mathrm{H}_{14} \mathrm{~N}_{2} \mathrm{O}[\mathrm{M}]^{+}:$190.11006; found 190.11019 .

\section{1-methyl-5-(5-(trifluoromethyl)pyridin-3-yl)pyrrolidin-2-one (54)}<smiles>CN1C(=O)CCC1c1cncc(C(F)(F)F)c1</smiles>

The compound was prepared according to the General Procedure 4 using 3-chloro-5-(trifluoromethyl)pyridine (36.2 mg, 0.2 mmol), mpg-CN (10.0 mg), $\mathrm{NiCl}_{2} \bullet$ glyme (2.2 mg, 5 mol\%), 2,2'bipyridine (1.6 mg $5 \mathrm{~mol} \%)$, 1-methylpyrrolidin-2-one $(1.0 \mathrm{~mL})$ and 2,6-lutidine (70 $\mu \mathrm{L} 0.6$ mmol). The reaction mixture was irradiated under nitrogen atmosphere for $72 \mathrm{~h}$ using blue LED $(455( \pm 15) \mathrm{nm})$. The reaction mixture was then subjected to the work-up procedure as described in the General Procedure 4. Purification was performed using flash chromatography on silica gel using PE/EtOAc solvent mixture as eluent to provide the title compound in 69\% yield (33.6 mg, $\left.\mathrm{C}_{1}: \mathrm{C}_{2}=2: 1\right) .{ }^{1} \mathrm{H}$ NMR $\left(400 \mathrm{MHz}, \mathrm{CDCl}_{3}\right) \delta 8.87(\mathrm{~s}, 1 \mathrm{H}), 8.70(\mathrm{~s}, 1 \mathrm{H}), 7.74(\mathrm{~s}, 1 \mathrm{H}), 4.73-$ $4.58(\mathrm{~m}, 1 \mathrm{H}), 2.70(\mathrm{~s}, 3 \mathrm{H}), 2.66-2.37(\mathrm{~m}, 3 \mathrm{H}), 1.95-1.81(\mathrm{~m}, 1 \mathrm{H}) .{ }^{13} \mathrm{C}$ NMR $(101 \mathrm{MHz}$, $\left.\mathrm{CDCl}_{3}\right) \delta 175.5,175.4,152.7,151.6,146.7(\mathrm{q}, J=4.7 \mathrm{~Hz}), 146.6(\mathrm{q}, J=4.1 \mathrm{~Hz}), 137.3,132.9$, 130.9 (q, $J=3.5 \mathrm{~Hz}), 127.5,124.7$ (q, $J=264.1 \mathrm{~Hz}), 124.6$ (q, $J=272.9 \mathrm{~Hz}), 62.0,46.8,43.9$, 30.6, 29.8, 28.5, 28.4, 17.9. ${ }^{19} \mathrm{~F}$ NMR $\left(377 \mathrm{MHz}, \mathrm{CDCl}_{3}\right) \delta$-62.92. EI-MS: $m / z$ calculated. for $\mathrm{C}_{11} \mathrm{H}_{11} \mathrm{~F}_{3} \mathrm{~N}_{2} \mathrm{O}[\mathrm{M}]^{+}:$244.08180; found 244.08172. 


\section{4-(4-acetylphenyl)-1,3-dimethyltetrahydropyrimidin-2(1H)-one (55)}<smiles>CC(=O)c1ccc(C2CCN(C)C(=O)N2C)cc1</smiles>

The compound was prepared according to the General Procedure 4 using 1-(4-bromophenyl)ethan-1-one (39.8 mg, 0.2 mmol), mpg-CN (10.0 mg), NiBr $2 \bullet$ glyme (3.2 mg, 5 mol\%), 2,2'bipyridine (1.6 mg $5 \mathrm{~mol} \%)$, 1,3-dimethyltetrahydropyrimidin-2(1H)one $(1.0 \mathrm{~mL})$ and 2,6-lutidine $(70 \mu \mathrm{L} 0.6 \mathrm{mmol})$. The reaction mixture was irradiated under nitrogen atmosphere for 48h using blue LED (455 ( \pm 15$) \mathrm{nm})$. The reaction mixture was then subjected to the work-up procedure as described in the General Procedure 4. Purification was performed using flash chromatography on silica gel using PE/EtOAc solvent mixture as eluent to provide the title compound in $63 \%$ yield $\left(31.0 \mathrm{mg}, \mathrm{C}_{1}: \mathrm{C}_{2}=8: 1\right.$ from crude NMR). ${ }^{1} \mathrm{H}$ NMR (400 MHz, $\left.\mathrm{CDCl}_{3}\right) \delta 7.95$ (d, $\left.J=8.3 \mathrm{~Hz}, 2 \mathrm{H}\right), 7.27(\mathrm{~d}, J=8.3 \mathrm{~Hz}, 2 \mathrm{H}), 4.55$ - 4.49 (m, 1H), 3.13 - $3.04(\mathrm{~m}, 2 \mathrm{H}), 2.98$ (s, 3H), 2.88 (s, 3H), 2.59 (s, 3H), 2.45 - 2.33 (m, 1H), $1.98-1.82(\mathrm{~m}, 1 \mathrm{H}) .{ }^{13} \mathrm{C}$ NMR (101 MHz, $\left.\mathrm{CDCl}_{3}\right) \delta 197.6,156.7,147.1,136.6,129.0$, 126.5, 61.3, 44.0, 36.0, 35.0, 29.6, 26.7 EI-MS: $\mathrm{m} / \mathrm{z}$ calculated. for $\mathrm{C}_{14} \mathrm{H}_{18} \mathrm{~N}_{2} \mathrm{O}_{2}[\mathrm{M}]^{+}$: 246.13628 ; found 246.13626 .

\section{1-(4-acetylbenzyl)-3-methyltetrahydropyrimidin-2(1H)-one (56)}<smiles>CC(=O)c1ccc(CN2CCCN(C)C2=O)cc1</smiles>

The compound was prepared according to the General Procedure 4 using 1-(4-chlorophenyl)ethan-1-one (30.8 mg, 0.2 mmol), mpg-CN (10.0 mg), NiCl $\bullet$ glyme (2.2 mg, 5 mol\%), 2,2'bipyridine (1.6 mg $5 \mathrm{~mol} \%$ ), 1,3-dimethyltetrahydropyrimidin2(1H)-one (256.4 mg, $2.0 \mathrm{mmol}), 2$, 6 -lutidine $(70 \mu \mathrm{L} 0.6 \mathrm{mmol})$ and $\mathrm{CH}_{3} \mathrm{CN}(0.8 \mathrm{~mL})$. The reaction mixture was irradiated under nitrogen atmosphere for $72 \mathrm{~h}$ using blue LED $(455( \pm 15)$ $\mathrm{nm})$. The reaction mixture was then subjected to the work-up procedure as described in the General Procedure 4. Purification was performed using flash chromatography on silica gel using PE/EtOAc solvent mixture as eluent to provide the title compound in $67 \%$ yield (32.9 mg, $\mathrm{C}_{1}: \mathrm{C}_{2}=>20: 1$ from crude NMR). ${ }^{1} \mathrm{H}$ NMR $\left(400 \mathrm{MHz}, \mathrm{CDCl}_{3}\right) \delta 7.90(\mathrm{~d}, J=8.2 \mathrm{~Hz}, 1 \mathrm{H})$, $7.36(\mathrm{~d}, J=8.2 \mathrm{~Hz}, 1 \mathrm{H}), 4.60$ (s, 2H), 3.28 (t, $J=6.0 \mathrm{~Hz}, 2 \mathrm{H}), 3.18$ (t, $J=6.0 \mathrm{~Hz}, 2 \mathrm{H}), 2.98$ (s, 3H), 2.58 (s, 3H), 1.94 (p, J=6.0 Hz, 2H). $\left.{ }^{13} \mathrm{C} \mathrm{NMR} \mathrm{(101} \mathrm{MHz,} \mathrm{CDCl}_{3}\right) \delta 197.9,156.6,144.5$, 136.2, 128.7, 127.9, 51.3, 48.0, 45.7, 36.0, 26.7, 22.4. EI-MS: $m / z$ calculated. for $\mathrm{C}_{14} \mathrm{H}_{18} \mathrm{~N}_{2} \mathrm{O}_{2}$ $[\mathrm{M}]^{+}:$246.13628; found 246.13679. 


\section{1-([1,1'-biphenyl]-4-ylmethyl)-3-methyltetrahydropyrimidin-2(1H)-one (57)}<smiles>CN1CCCN(Cc2ccc(-c3ccccc3)cc2)C1=O</smiles>

The compound was prepared according to the General Procedure 4 using 4-chloro-1,1'-biphenyl (37.6 mg, $0.2 \mathrm{mmol}$ ), mpg-CN (10.0 mg), $\mathrm{NiCl}_{2} \bullet \mathrm{glyme}(2.2 \mathrm{mg}, 5 \mathrm{~mol} \%), 2,2^{\prime}-$ bipyridine (1.6 mg 5 mol\%), 1,3-dimethyltetrahydropyrimidin2(1H)-one (256.4 mg, $2.0 \mathrm{mmol})$, 2,6-lutidine ( $70 \mu \mathrm{L} 0.6 \mathrm{mmol})$ and $\mathrm{CH}_{3} \mathrm{CN}(0.8 \mathrm{~mL})$. The reaction mixture was irradiated under nitrogen atmosphere for $72 \mathrm{~h}$ using blue LED $(455( \pm 15)$ $\mathrm{nm})$. The reaction mixture was then subjected to the work-up procedure as described in the General Procedure 4. Purification was performed using flash chromatography on silica gel using PE/EtOAc solvent mixture as eluent to provide the title compound in $66 \%$ yield (37.0 $\mathrm{mg}, \mathrm{C}_{1}: \mathrm{C}_{2}=>20: 1$ from crude NMR). ${ }^{1} \mathrm{H}$ NMR $\left(400 \mathrm{MHz}, \mathrm{CDCl}_{3}\right) \delta 7.61-7.52(\mathrm{~m}, 4 \mathrm{H}), 7.48$ $-7.39(\mathrm{~m}, 2 \mathrm{H}), 7.38-7.31(\mathrm{~m}, 3 \mathrm{H}), 4.60(\mathrm{~s}, 2 \mathrm{H}), 3.28(\mathrm{t}, J=6.0 \mathrm{~Hz}, 2 \mathrm{H}), 3.22(\mathrm{t}, J=6.0 \mathrm{~Hz}$, 2H), 3.00 (s, 3H), 1.95 (p, J=5.9 Hz, 2H). $\left.{ }^{13} \mathrm{C} \mathrm{NMR} \mathrm{(101} \mathrm{MHz,} \mathrm{CDCl}_{3}\right) \delta$ 156.7, 141.1, 140.1, 137.8, 128.8, 128.4, 127.3, 127.3, 127.2, 51.1, 48.0, 45.4, 36.0, 22.4. EI-MS: m/z calculated. for $\mathrm{C}_{18} \mathrm{H}_{20} \mathrm{~N}_{2} \mathrm{O}[\mathrm{M}]^{+}: 280.15701$; found 280.15639 .

\section{4-([1,1'-biphenyl]-4-yl)-1,3-dimethyltetrahydropyrimidin-2(1H)-one (58)}

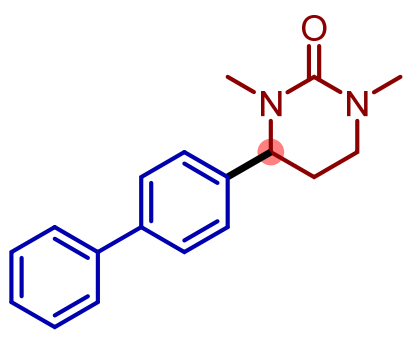

The compound was prepared according to the General Procedure 4 using 4-bromo-1,1'-biphenyl (46.6 mg, $0.2 \mathrm{mmol}$ ), mpg-CN (10.0 mg), NiBr2•glyme (3.2 mg, 5 mol\%), 2,2'-bipyridine (1.6 mg $5 \mathrm{~mol} \%)$, 1,3-dimethyltetrahydropyrimidin-2(1H)-one (1.0 $\mathrm{mL})$ and 2,6-lutidine $(70 \mu \mathrm{L} 0.6 \mathrm{mmol})$. The reaction mixture was irradiated under nitrogen atmosphere for 48h using blue LED (455 $( \pm 15) \mathrm{nm})$. The reaction mixture was then subjected to the work-up procedure as described in the General Procedure 4. Purification was performed using flash chromatography on silica gel using PE/EtOAc solvent mixture as eluent to provide the title compound in $61 \%$ yield $\left(34.2 \mathrm{mg}, \mathrm{C}_{1}: \mathrm{C}_{2}=7.9: 1\right.$ from crude NMR). ${ }^{1} \mathrm{H}$ NMR (400 MHz, $\left.\mathrm{CDCl}_{3}\right) \delta 7.60-7.54(\mathrm{~m}, 4 \mathrm{H}), 7.47-7.41(\mathrm{~m}, 2 \mathrm{H}), 7.37$ $7.31(\mathrm{~m}, 1 \mathrm{H}), 7.27-7.20(\mathrm{~m}, 2 \mathrm{H}), 4.51$ (t, $J=4.4 \mathrm{~Hz}, 1 \mathrm{H}), 3.18(\mathrm{td}, J=11.5,3.8 \mathrm{~Hz}, 1 \mathrm{H}), 3.10$ $-3.04(\mathrm{~m}, 1 \mathrm{H}), 2.39$ (ddt, $J=13.2,10.6,5.1 \mathrm{~Hz}, 1 \mathrm{H}), 1.92(\mathrm{dq}, J=13.2,3.8 \mathrm{~Hz}, 1 \mathrm{H}) .{ }^{13} \mathrm{C} \mathrm{NMR}$ $\left(101 \mathrm{MHz} \mathrm{CDCl}_{3}\right) \delta 156.9,140.7,140.6,140.5,128.8,127.5,127.4,127.1,126.7,61.14,44.1$, 35.9, 34.9, 29.9. EI-MS: $m / z$ calculated. for $\mathrm{C}_{18} \mathrm{H}_{20} \mathrm{~N}_{2} \mathrm{O}[\mathrm{M}]^{+}: 280.15701$; found 280.15667. 


\section{Hexamethylphosphoramide derivative (59)}

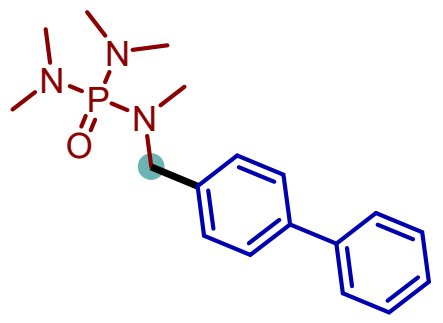

The compound was prepared according to the General Procedure 4 using 4-bromo-1,1'-biphenyl (46.6 mg, $0.2 \mathrm{mmol}$ ), mpg-CN (10.0 mg), NiBr2•glyme (3.2 mg, 5 mol\%), 2,2'-bipyridine (1.6 mg $5 \mathrm{~mol} \%)$, hexamethylphosphoramide $(1.0 \mathrm{~mL})$ and 2,6lutidine $(70 \mu \mathrm{L} 0.6 \mathrm{mmol})$. The reaction mixture was irradiated under nitrogen atmosphere for $72 \mathrm{~h}$ using blue LED $(455( \pm 15) \mathrm{nm})$. The reaction mixture was then subjected to the work-up procedure as described in the General Procedure 4. Purification was performed using flash chromatography on silica gel using PE/EtOAc solvent mixture as eluent to provide the title compound in $43 \%$ yield $(28.4 \mathrm{mg}) .{ }^{1} \mathrm{H}$ NMR $\left(400 \mathrm{MHz}, \mathrm{CDCl}_{3}\right) \delta$ $7.62-7.54(\mathrm{~m}, 4 \mathrm{H}), 7.46-7.39(\mathrm{~m}, 4 \mathrm{H}), 7.36-7.31(\mathrm{~m}, 1 \mathrm{H}), 4.19(\mathrm{~d}, J=9.1 \mathrm{~Hz}, 2 \mathrm{H}), 2.71$ (s, 6H), $2.69(\mathrm{~s}, 6 \mathrm{H}), 2.58(\mathrm{~d}, J=9.1 \mathrm{~Hz}, 3 \mathrm{H}) .{ }^{13} \mathrm{C} \mathrm{NMR}\left(101 \mathrm{MHz}, \mathrm{CDCl}_{3}\right) \delta 141.0,140.2$, 137.7, 137.6, 128.9, 128.6, 127.3, 127.2, 127.1, 53.0, 52.9, 37.1, 37.0, 34.2, 34.1. ${ }^{31} \mathrm{P}$ NMR $\left(162 \mathrm{MHz}, \mathrm{CDCl}_{3}\right) \delta$-62.92. HRMS (ESI): $\mathrm{m} / \mathrm{z}$ calculated. for $\mathrm{C}_{18} \mathrm{H}_{27} \mathrm{~N}_{3} \mathrm{OP}[\mathrm{M}+\mathrm{H}]^{+}: 332.1886$; found 332.1890 .

\section{Methyl (R)-2-(4-((N-methylacetamido)methyl)benzamido)-2-phenylacetate (60)}

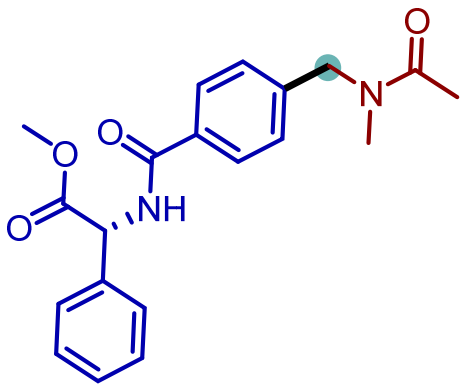

The compound was prepared according to the General Procedure 1 methyl (R)-2-(4-bromobenzamido)-2phenylacetate $^{14}$ (69.6 mg, $\left.0.2 \mathrm{mmol}\right), \operatorname{mpg}-\mathrm{CN}(10.0 \mathrm{mg})$, NiBr2•glyme (3.2 mg, 5 mol\%), 2,2'-bipyridine (1.6 mg $5 \mathrm{~mol} \%$ ), $N, N$-dimethylacetamide $(1.0 \mathrm{~mL})$ and 2,6 -lutidine $(70 \mu \mathrm{L} 0.6$ mmol). The reaction mixture was irradiated under nitrogen atmosphere for $72 \mathrm{~h}$ using blue LED $(455( \pm 15) \mathrm{nm})$. The reaction mixture was then subjected to the work-up procedure as described in the General Procedure 1. Purification was performed using flash chromatography on silica gel using PE/EtOAc solvent mixture as eluent to provide the title compound in $91 \%$ yield $(64.4 \mathrm{mg}) .{ }^{1} \mathrm{H}$ NMR $\left(400 \mathrm{MHz}, \mathrm{CDCl}_{3}\right) \delta 7.81(\mathrm{~d}, J=8.2 \mathrm{~Hz})$ \& $7.75(\mathrm{~d}, J=8.2 \mathrm{~Hz}, 2 \mathrm{H}$ combined), $7.44-7.27$ (m, 6H), $7.23(\mathrm{~d}, J=8.2 \mathrm{~Hz}) \& 7.17$ (d, $J=$ $8.2 \mathrm{~Hz}, 2 \mathrm{H}$ combined), $5.74(\mathrm{~d}, J=6.9 \mathrm{~Hz}) \& 5.73(\mathrm{~d}, J=6.9 \mathrm{~Hz}, 1 \mathrm{H}$ combined), 4.55 (s) \& $4.50(\mathrm{~s}, 2 \mathrm{H}$ combined), $3.71(\mathrm{~s}, 2 \mathrm{H}), 2.87(\mathrm{~s}) \& 2.86(\mathrm{~s}, 3 \mathrm{H}$ combined), 2.09 (s) \& 2.06 (s, 3H combined). ${ }^{13} \mathrm{C}$ NMR $\left(101 \mathrm{MHz}, \mathrm{CDCl}_{3}\right) \delta 171.4(\mathrm{x} 2), 170.9 \& 170.8,166.3 \& 166.1,141.4 \&$ $140.6,136.4 \& 136.3,133.0 \& 132.6,129.0 \& 128.9,128.5 \& 128.5,127.9$ (x2), $127.5 \& 127.4$, $126.3(\mathrm{x} 2), 56.8,52.8,53.9 \& 50.3,35.6 \& 33.7,21.6 \& 21.3$. HRMS (ESI): $\mathrm{m} / \mathrm{z}$ calculated. for $\mathrm{C}_{20} \mathrm{H}_{23} \mathrm{~N}_{2} \mathrm{O}_{4}[\mathrm{M}+\mathrm{H}]^{+}:$355.1652; found 355.1659. 


\section{Isopropyl 2-methyl-2-(4-(4-(( $N$-methylacetamido)methyl)benzoyl)phenoxy)propanoate}

(61)<smiles>CC(=O)N(C)Cc1ccc(C(=O)c2ccc(OC(C)(C)C(=O)OC(C)C)cc2)cc1</smiles>
The compound was prepared according to the General Procedure 2 using fenofibrate (72.0 mg, $0.2 \mathrm{mmol})$, mpg-CN (10.0 mg), $\mathrm{NiCl}_{2} \bullet \mathrm{glyme}$ (2.2 mg, $5 \mathrm{~mol} \%$ ), 2,2'-bipyridine (1.6 mg 5 mol\%), $N, N$-dimethylacetamide $(1.0 \mathrm{~mL})$ and 2,6-lutidine $(70 \mu \mathrm{L} 0.6 \mathrm{mmol})$. The reaction mixture was irradiated under nitrogen atmosphere for $72 \mathrm{~h}$ using blue LED $(455( \pm 15) \mathrm{nm})$. The reaction mixture was then subjected to the work-up procedure as described in the General Procedure 2. Purification was performed using flash chromatography on silica gel using $\mathrm{PE} /$ EtOAc solvent mixture as eluent to provide the title compound in $83 \%$ yield $(68.2 \mathrm{mg}) .{ }^{1} \mathrm{H}$ NMR (400 MHz, $\left.\mathrm{CDCl}_{3}\right) \delta 7.74(\mathrm{~d}, J=8.1 \mathrm{~Hz}, 1 \mathrm{H}) \& 7.69(\mathrm{~d}, J=8.1 \mathrm{~Hz}, 2 \mathrm{H}$ combined), 7.73 $(\mathrm{d}, J=8.8 \mathrm{~Hz}) \& 7.72(\mathrm{~d}, J=8.8 \mathrm{~Hz}, 2 \mathrm{H}$ combined), $7.31(\mathrm{~d}, J=8.1 \mathrm{~Hz}) \& 7.26(\mathrm{~d}, J=8.1 \mathrm{~Hz}$, 2H combined), 6.85 (d, $J=8.8 \mathrm{~Hz}) \& 6.84(\mathrm{~d}, J=8.8 \mathrm{~Hz}, 2 \mathrm{H}$ combined), 5.06 (hept, $J=6.3$ Hz, 1H), 4.64 (s) \& 4.59 (s, 2H combined), 2.95 (s, 3H), 2.16 (s) \& 2.14 (s, 3H combined), 1.64 $(\mathrm{s}, 6 \mathrm{H}), 1.18(\mathrm{~d}, J=6.3 \mathrm{~Hz}, 6 \mathrm{H}) .{ }^{13} \mathrm{C} \mathrm{NMR}\left(101 \mathrm{MHz}, \mathrm{CDCl}_{3}\right) \delta 195.2 \& 194.8,173.2 \& 173.1$, $171.0 \& 170.9,159.7 \& 159.6,141.7 \& 140.8,137.7 \& 137.3,132.1,130.6 \& 130.4,130.5 \&$ $130.2,127.7 \& 126.1,117.3 \& 117.2,79.4 \& 79.4,69.3,54.1 \& 50.6,35.9 \& 34.0,25.4,21.6$, 21.8 \& 21.5. HRMS (ESI): $m / z$ calculated. for $\mathrm{C}_{24} \mathrm{H}_{30} \mathrm{NO}_{5}[\mathrm{M}+\mathrm{H}]^{+}: 412.2118$; found 412.2124 .

\section{$N$-methyl- $N$-((1,3,7-trimethyl-2,6-dioxo-2,3,6,7-tetrahydro-1H-purin-8-}

yl)methyl)acetamide (62)

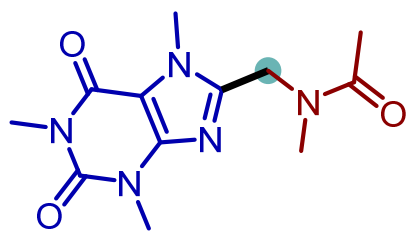

The compound was prepared according to the General Procedure 2 methyl 8-bromo caffeine (54.6 $\mathrm{mg}, 0.2 \mathrm{mmol}$ ), tetrabutylammonium chloride $(66.7 \mathrm{mg}, 0.24 \mathrm{mmol}), \mathrm{mpg}-\mathrm{CN}(10.0$ $\mathrm{mg}$ ), $\mathrm{NiCl}_{2} \bullet$ glyme (2.2 mg, $5 \mathrm{~mol} \%$ ), 2,2'-bipyridine (1.6 mg 5 mol\%), $N, N$-dimethylacetamide $(1.0 \mathrm{~mL})$ and 2,6-lutidine $(70 \mu \mathrm{L} 0.6 \mathrm{mmol})$. The reaction mixture was irradiated under nitrogen atmosphere for $72 \mathrm{~h}$ using blue LED $(455( \pm 15) \mathrm{nm})$. The reaction mixture was then subjected to the work-up procedure as described in the General Procedure 2. Purification was performed using flash chromatography on silica gel using $\mathrm{PE} /$ EtOAc solvent mixture as eluent to provide the title compound in $72 \%$ yield $(40.2 \mathrm{mg}) .{ }^{1} \mathrm{H}$ NMR (400 MHz, $\left.\mathrm{CDCl}_{3}\right) \delta 4.67$ (s) \& 4.57 (s, 2H combined), 3.99 (s) \& 3.96 (s, 3H combined), $3.54(\mathrm{~s}) \& 3.53(\mathrm{~s}, 3 \mathrm{H}$ combined), 3.37 (s, 3H) $3.10(\mathrm{~s}) \& 3.00(\mathrm{~s}, 3 \mathrm{H}$ combined), 2.12 (s) \& 2.09 (s, 3H combined). ${ }^{13} \mathrm{C}$ NMR $\left(101 \mathrm{MHz}, \mathrm{CDCl}_{3}\right) \delta 170.8,155.5,151.7,149.2,147.7,108.2$, 
42.2, 36.1, 32.4, 29.8, 28.0, 21.6. HRMS (ESI): $\mathrm{m} / \mathrm{z}$ calculated. for $\mathrm{C}_{12} \mathrm{H}_{18} \mathrm{~N}_{5} \mathrm{O}_{3}[\mathrm{M}+\mathrm{H}]^{+}$: 280.1404; found 280.1409.

$N$-(3-chloro-5-(1,5-dimethyl-2,4-dioxo-3-azabicyclo[3.1.0]hexan-3-yl)benzyl)- $N$ methylacetamide (63)<smiles>CC(=O)N(C)Cc1cc(Cl)cc(N2C(=O)C3(C)CC3(C)C2=O)c1</smiles>

The compound was prepared according to the General Procedure 2 using procymidone $(56.8 \mathrm{mg}, 0.2 \mathrm{mmol})$, mpg-CN (10.0 $\mathrm{mg}$ ), $\mathrm{NiCl}_{2} \bullet$ glyme $(2.2 \mathrm{mg}, 5 \mathrm{~mol} \%$ ), 2,2'-bipyridine (1.6 mg $5 \mathrm{~mol} \%$ ), $N, N$-dimethylacetamide $(1.0 \mathrm{~mL})$ and 2,6-lutidine $(70 \mu \mathrm{L} 0.6 \mathrm{mmol})$. The reaction mixture was irradiated under nitrogen atmosphere for $72 \mathrm{~h}$ using blue LED $(455( \pm 15) \mathrm{nm})$. The reaction mixture was then subjected to the work-up procedure as described in the General Procedure 2. Purification was performed using flash chromatography on silica gel using PE/EtOAc solvent mixture as eluent to provide the title compound in 67\% yield (44.7 mg). ${ }^{1} \mathrm{H}$ NMR (400 MHz, $\left.\mathrm{CDCl}_{3}\right) \delta 7.22-7.09(\mathrm{~m}, 2 \mathrm{H}), 7.02$ (s) \& 6.94 (s, 1H combined), 4.53 (s) \& 4.49 (s, 2H combined), 2.92 (s) \& 2.92 (s, 3H combined), $2.13(\mathrm{~s}) \& 2.11(\mathrm{~s}, 3 \mathrm{H}$ combined), $1.74(\mathrm{dd}, J=9.6,4.7 \mathrm{~Hz}, 1 \mathrm{H}), 1.49$ (s) \& 1.47 (s, 6H combined), $1.18(\mathrm{dd}, J=9.6,4.7 \mathrm{~Hz}, 1 \mathrm{H}) .{ }^{13} \mathrm{C} \mathrm{NMR}\left(101 \mathrm{MHz}, \mathrm{CDCl}_{3}\right) \delta 176.3 \& 176.2,171.1$ $\& 170.9,140.1 \& 139.4,135.3 \& 134.7,133.6 \& 133.2,127.7 \& 125.9,125.7 \& 125.5,124.3$ $\& 122.4,53.6 \& 50.0,35.7 \& 33.9,33.0 \& 32.9,30.2 \& 30.1,21.8 \& 21.5,10.0$. HRMS (ESI): $m / z$ calculated. for $\mathrm{C}_{17} \mathrm{H}_{19} \mathrm{ClN}_{2} \mathrm{O}_{3}[\mathrm{M}+\mathrm{H}]^{+}: 335.1157$; found 335.1162 .

(1R,2S,5R)-2-isopropyl-5-methylcyclohexyl 4-(( $N$-methylacetamido)methyl)benzoate (64)

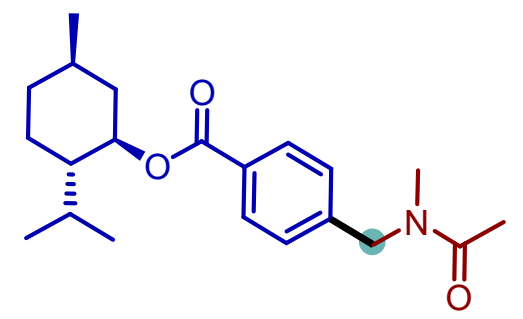

The compound was prepared according to the General Procedure 1 (1R,2S,5R)-2-isopropyl-5-methylcyclohexyl 4bromobenzoate $^{14}(67.8 \mathrm{mg}, 0.2 \mathrm{mmol}), \operatorname{mpg}-\mathrm{CN}(10.0 \mathrm{mg})$, $\mathrm{NiBr}_{2} \bullet$ glyme (3.2 mg, $5 \mathrm{~mol} \%$ ), 2,2'-bipyridine (1.6 mg 5 mol\%), $N, N$-dimethylacetamide $(1.0 \mathrm{~mL})$ and 2,6-lutidine $(70$

$\mu \mathrm{L} 0.6 \mathrm{mmol})$. The reaction mixture was irradiated under nitrogen atmosphere for $48 \mathrm{~h}$ using blue LED $(455( \pm 15) \mathrm{nm})$. The reaction mixture was then subjected to the work-up procedure as described in the General Procedure 1. Purification was performed using flash chromatography on silica gel using PE/EtOAc solvent mixture as eluent to provide the title compound in $79 \%$ yield $(54.5 \mathrm{mg}) .{ }^{1} \mathrm{H} \mathrm{NMR}\left(400 \mathrm{MHz}, \mathrm{CDCl}_{3}\right) \delta 8.01(\mathrm{~d}, J=8.2 \mathrm{~Hz}) \& 7.96$ (d, $J=8.2 \mathrm{~Hz}, 2 \mathrm{H}$ combined), $7.26(\mathrm{~d}, J=8.2 \mathrm{~Hz}) \& 7.21$ (d, $J=8.2 \mathrm{~Hz}, 2 \mathrm{H}$ combined), 4.96 $-4.77(\mathrm{~m}, 1 \mathrm{H}), 4.67-4.51(\mathrm{~m}, 2 \mathrm{H}), 2.91(\mathrm{~s}) \& 2.90$ (s, 3H combined), 2.12 (s) \& 2.09 (s, 3H 
combined), $2.08-2.02(\mathrm{~m}, 1 \mathrm{H}), 1.97-1.84(\mathrm{~m}, 1 \mathrm{H}), 1.75-1.61(\mathrm{~m}, 2 \mathrm{H}), 1.58-1.41(\mathrm{~m}, 2 \mathrm{H})$, $1.15-0.97(\mathrm{~m}, 2 \mathrm{H}), 0.91-0.84(\mathrm{~m}, 7 \mathrm{H}), 0.75(\mathrm{~d}, J=6.9 \mathrm{~Hz}) \& 0.74(\mathrm{~d}, J=6.9 \mathrm{~Hz}, 3 \mathrm{H}$ combined). ${ }^{13} \mathrm{C} \mathrm{NMR}\left(101 \mathrm{MHz}, \mathrm{CDCl}_{3}\right) \delta 170.9 \& 170.7,165.8 \& 165.6,142.4 \& 141.6,130.4$ $\& 130.2,130.0 \& 129.9,127.8 \& 126.2,74.9 \& 74.8,54.1 \& 50.4,47.2,40.9,34.3,35.72 \&$ 33.8, 31.4, 26.6, 26.5, 23.6, 22.0, $21.7 \& 21.4,20.7$, 16.5. HRMS (ESI): $m / z$ calculated. for $\mathrm{C}_{21} \mathrm{H}_{32} \mathrm{NO}_{3}[\mathrm{M}+\mathrm{H}]^{+}: 346.2377$; found 346.2381.

$(3 S, 8 S, 9 S, 10 R, 13 R, 14 S, 17 R)-10,13-d i m e t h y l-17-((R)-6-m e t h y l h e p t a n-2-y l)-$

$2,3,4,7,8,9,10,11,12,13,14,15,16,17-t e t r a d e c a h y d r o-1 H$-cyclopenta[a]phenanthren-3-yl 4((N-methylacetamido)methyl)benzoate (65)

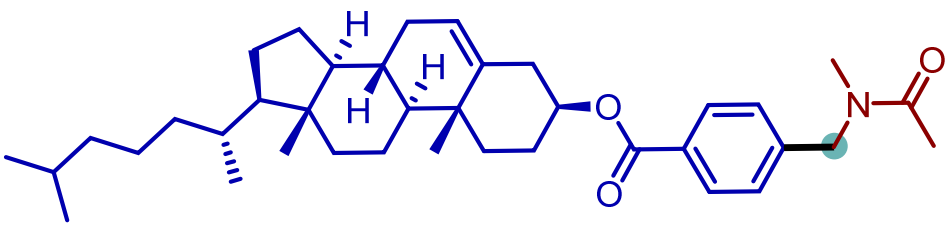

The compound was prepared according to the General Procedure 1 $(3 S, 8 S, 9 S, 10 R, 13 R, 14 S, 17 R)-$ 10,13-dimethyl-17-((R)-6-methylheptan-2-yl)-2,3,4,7,8,9,10,11,12,13,14,15,16,17-

tetradecahydro-1H-cyclopenta[a]phenanthren-3-yl 4-bromobenzoate ${ }^{14}$ (113.8 mg, $\left.0.2 \mathrm{mmol}\right)$, mpg-CN (10.0 mg), NiBr2•glyme (3.2 mg, 5 mol\%), 2,2'-bipyridine (1.6 mg 5 mol\%), $N, N$ dimethylacetamide $(1.0 \mathrm{~mL})$ and 2,6-lutidine $(70 \mu \mathrm{L} 0.6 \mathrm{mmol})$. The reaction mixture was irradiated under nitrogen atmosphere for 48h using blue LED (455 $( \pm 15) \mathrm{nm})$. The reaction mixture was then subjected to the work-up procedure as described in the General Procedure 1. Purification was performed using flash chromatography on silica gel using PE/EtOAc solvent mixture as eluent to provide the title compound in $71 \%$ yield $(81.6 \mathrm{mg}) .{ }^{1} \mathrm{H}$ NMR $(400 \mathrm{MHz}$, $\left.\mathrm{CDCl}_{3}\right) \delta 8.04(\mathrm{~d}, J=8.2 \mathrm{~Hz}) \& 7.99(\mathrm{~d}, J=8.2 \mathrm{~Hz}, 2 \mathrm{H}$ combined), $7.29(\mathrm{~d}, J=8.2 \mathrm{~Hz}) \& 7.23$ (d, $J=8.2 \mathrm{~Hz}, 2 \mathrm{H}$ combined), 5.41 (dd, $J=4.9,2.4 \mathrm{~Hz}, 1 \mathrm{H}), 4.92-4.76(\mathrm{~m}, 1 \mathrm{H}), 4.63$ (s) \& 4.57 (s, 2H combined), 2.94 (s) \& 2.92 (s, 3H combined), 2.45 (d, J=7.6 Hz, 2H), 2.17 (s) \& $2.13(\mathrm{~s}, 3 \mathrm{H}$ combined), $2.08-1.67(\mathrm{~m}, 6 \mathrm{H}), 1.62-1.08(\mathrm{~m}, 17 \mathrm{H}), 1.06(\mathrm{~s}, 3 \mathrm{H}), 1.05-0.96(\mathrm{~m}$, $3 \mathrm{H}), 0.92(\mathrm{~d}, J=6.5 \mathrm{~Hz}, 3 \mathrm{H}), 0.86(\mathrm{dd}, J=6.6,1.7 \mathrm{~Hz}, 6 \mathrm{H}), 0.68(\mathrm{~s}, 3 \mathrm{H}) .{ }^{3} \mathrm{C}$ NMR $(101 \mathrm{MHz}$, $\left.\mathrm{CDCl}_{3}\right) \delta 171.1 \& 170.9,165.8 \& 165.6,142.5 \& 141.7,139.8 \& 139.7,130.5 \&, 130.4,130.1$ $\& 130.0,127.9 \& 126.2,123.0 \& 122.9,74.8,74.7,56.8,56.3,54.2 \& 50.6,50.2,42.4,39.8$, 39.6, 38.3, 37.2, 36.8, 36.3, 35.9, $35.7 \& 34.0,32.1$, 32.0, 28.3, 28.1, 28.0, 24.4, 23.9, 22.9, 22.7, $21.9 \& 21.5,21.2,19.5,18.8,12.0$. HRMS (ESI): $m / z$ calculated. for $\mathrm{C}_{38} \mathrm{H}_{58} \mathrm{NO}_{3}[\mathrm{M}+\mathrm{H}]^{+}$: 576.4411 ; found 576.4407 . 


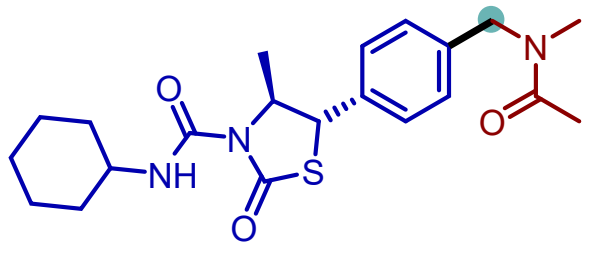

The compound was prepared according to the General Procedure 2 using hexythiazox $(70.4 \mathrm{mg}, 0.2$ mmol), mpg-CN (10.0 mg), $\mathrm{NiCl}_{2} \bullet$ glyme (2.2 mg, 5 mol\%), 2,2'-bipyridine (1.6 mg 5 mol\%), N,Ndimethylacetamide $(1.0 \mathrm{~mL})$ and 2,6-lutidine $(70 \mu \mathrm{L} 0.6 \mathrm{mmol})$. The reaction mixture was irradiated under nitrogen atmosphere for $72 \mathrm{~h}$ using blue LED (455 $( \pm 15) \mathrm{nm})$. The reaction mixture was then subjected to the work-up procedure as described in the General Procedure 2. Purification was performed using flash chromatography on silica gel using $\mathrm{PE} / \mathrm{EtOAc}_{\mathrm{E}} \mathrm{Et}_{3} \mathrm{~N}$ solvent mixture as eluent to provide the title compound in 68\% yield (54.8 mg). ${ }^{1} \mathrm{H}$ NMR (400 $\left.\mathrm{MHz}, \mathrm{CDCl}_{3}\right) \delta 8.09(\mathrm{~d}, J=7.8 \mathrm{~Hz}, 1 \mathrm{H}), 7.34-7.11(\mathrm{~m}, 4 \mathrm{H}), 4.88-4.81(\mathrm{~m}, 1 \mathrm{H}), 4.55(\mathrm{~s}) \&$ $4.51(\mathrm{~s}, 2 \mathrm{H}), 4.20$ (s) \& $4.18(\mathrm{~s}, 1 \mathrm{H}$ combined), $3.75-3.62(\mathrm{~m}, 1 \mathrm{H}), 2.92(\mathrm{~s}, 3 \mathrm{H}), 2.15$ (s) \& 2.13 (s, 3H combined), $1.98-1.86(\mathrm{~m}, 2 \mathrm{H}), 1.75-1.65(\mathrm{~m}, 2 \mathrm{H}), 1.63-1.53(\mathrm{~m}, 4 \mathrm{H}), 1.43-$ $1.18(\mathrm{~m}, 5 \mathrm{H}) .{ }^{13} \mathrm{C}$ NMR $\left(101 \mathrm{MHz}, \mathrm{CDCl}_{3}\right) \delta 173.1 \& 172.9,171.1 \& 170.9,150.4,140.6 \&$ $140.2,137.8 \& 137.0,128.9 \& 127.3,127.1 \& 126.9,62.1,53.9 \& 50.3,50.7 \& 50.6,49.2,35.8$ $\& 33.9,33.1 \& 33.0,25.6,24.7,21.9 \&$ 21.5, 20.5. HRMS (ESI): $\mathrm{m} / \mathrm{z}$ calculated. for $\mathrm{C}_{21} \mathrm{H}_{30} \mathrm{~N}_{3} \mathrm{O}_{3} \mathrm{~S}[\mathrm{M}+\mathrm{H}]^{+}:$404.2002; found 404.2011.

\section{$N$-methyl- $N$-(4-(3-methyl-1,1-dioxido-4-oxo-1,3-thiazinan-2-yl)benzyl)acetamide (67)}

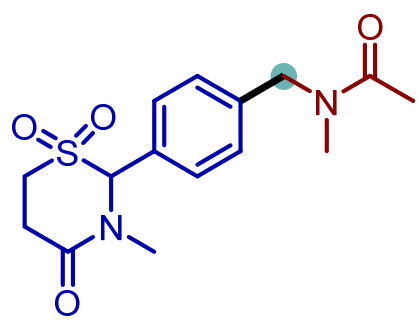

The compound was prepared according to the General Procedure 2 using chlormezanone (54.6 mg, $0.2 \mathrm{mmol}$ ), mpg-CN (10.0 mg), $\mathrm{NiCl}_{2} \bullet$ glyme (2.2 mg, $5 \mathrm{~mol} \%$ ), 2,2'-bipyridine (1.6 mg 5 mol\%), $N, N$-dimethylacetamide $(1.0 \mathrm{~mL})$ and 2,6-lutidine $(70 \mu \mathrm{L}$ $0.6 \mathrm{mmol}$ ). The reaction mixture was irradiated under nitrogen atmosphere for $72 \mathrm{~h}$ using blue LED $(455( \pm 15) \mathrm{nm})$. The reaction mixture was then subjected to the work-up procedure as described in the General Procedure 2. Purification was performed using flash chromatography on silica gel using PE/EtOAc solvent mixture as eluent to provide the title compound in $76 \%$ yield (49.2 mg). ${ }^{1} \mathrm{H} \mathrm{NMR}\left(400 \mathrm{MHz}, \mathrm{CDCl}_{3}\right) \delta 7.35-7.16(\mathrm{~m}, 4 \mathrm{H})$, $5.24-5.18(\mathrm{~m}, 1 \mathrm{H}), 4.63-4.36(\mathrm{~m}, 2 \mathrm{H}), 3.34-2.97(\mathrm{~m}, 4 \mathrm{H}), 2.90(\mathrm{~s}) \& 2.87$ (s, 3H combined), $2.87(\mathrm{~s}) \& 2.86\left(\mathrm{~s}, 3 \mathrm{H}\right.$ combined), 2.09 (s) \& 2.06 (s, 3H combined). ${ }^{13} \mathrm{C}$ NMR $(101 \mathrm{MHz}$, $\left.\mathrm{CDCl}_{3}\right) \delta 171.1 \& 171.0,166.2,140.2 \& 139.5,129.6 \& 129.1,128.9 \& 128.7,128.5 \& 127.2$, $80.5 \& 80.4,53.4 \& 50.5,43.7 \& 43.5,36.3 \& 36.2,36.2 \& 34.0,30.6,21.8 \& 21.5$. HRMS (ESI): $m / z$ calculated. for $\mathrm{C}_{15} \mathrm{H}_{21} \mathrm{~N}_{2} \mathrm{O}_{4} \mathrm{~S}[\mathrm{M}+\mathrm{H}]^{+}: 325.1217$; found 325.1219 . 
Ethyl 4-(8-((N-methylacetamido)methyl)-5,6-dihydro-11H-benzo[5,6]cyclohepta[1,2b]pyridin-11-ylidene)piperidine-1-carboxylate (68)

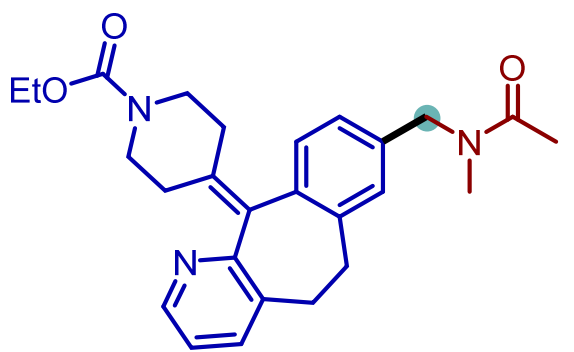

The compound was prepared according to the General Procedure 2 using loratadine (76.4 mg, $0.2 \mathrm{mmol})$,

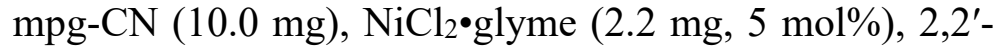
bipyridine (1.6 mg $5 \mathrm{~mol} \%), N, N$-dimethylacetamide (1.0 $\mathrm{mL})$ and 2,6-lutidine $(70 \mu \mathrm{L} 0.6 \mathrm{mmol})$. The reaction mixture was irradiated under nitrogen atmosphere for $72 \mathrm{~h}$ using blue LED $(455( \pm 15) \mathrm{nm})$. The reaction mixture was then subjected to the work-up procedure as described in the General Procedure 2. Purification was performed using flash chromatography on silica gel using $\mathrm{PE} / \mathrm{EtOAc} / \mathrm{Et}_{3} \mathrm{~N}$ solvent mixture as eluent to provide the title compound in $63 \%$ yield $(54.5$ mg). ${ }^{1} \mathrm{H}$ NMR $\left(400 \mathrm{MHz}, \mathrm{CDCl}_{3}\right) \delta 8.40\left(\mathrm{dd}, J_{1}=J_{2}=4.0 \mathrm{~Hz}, 1 \mathrm{H}\right), 7.44\left(\mathrm{dd}, J_{1}=J_{2}=6.4 \mathrm{~Hz}\right.$, 1H), $7.22-6.90(\mathrm{~m}, 4 \mathrm{H}), 4.51$ (s) \& 4.47 (s, 2H combined), 4.13 (q, J = 7.1 Hz, 2H), $3.94-$ $3.67(\mathrm{~m}, 2 \mathrm{H}), 3.47-3.25(\mathrm{~m}, 2 \mathrm{H}), 3.20-3.05(\mathrm{~m}, 2 \mathrm{H}), 2.92(\mathrm{~s}, 3 \mathrm{H}), 2.90-2.72(\mathrm{~m}, 2 \mathrm{H}), 2.58$ $-2.20(\mathrm{~m}, 4 \mathrm{H}), 2.14(\mathrm{~s}) \& 2.13\left(\mathrm{~s}, 3 \mathrm{H}\right.$ combined), $1.24(\mathrm{t}, J=7.1 \mathrm{~Hz}, 3 \mathrm{H}) .{ }^{13} \mathrm{C} \mathrm{NMR}(101 \mathrm{MHz}$, $\left.\mathrm{CDCl}_{3}\right) \delta 171.0 \& 170.7,157.6 \& 157.5,155.5,146.7 \& 146.6,138.6 \& 138.4,138.3 \& 138.0$, $137.5 \& 137.4,137.1 \& 136.8,136.6 \& 135.7,135.10 \& 134.8,133.7 \& 133.6,129.9 \& 129.5$, $128.7 \& 126.8,125.6 \& 124.1,122.2 \& 122.1,61.3,54.0 \& 50.4,44.9 \& 44.8,35.81 \& 33.8$, $32.0 \& 31.9,31.7 \& 31.6,30.8 \& 30.6,21.9 \& 21.5,14.7$. HRMS (ESI): $\mathrm{m} / \mathrm{z}$ calculated. for $\mathrm{C}_{26} \mathrm{H}_{32} \mathrm{~N}_{3} \mathrm{O}_{3}[\mathrm{M}+\mathrm{H}]^{+}:$434.2438; found 434.2444.

(3aR,5R,6S,6aR)-5-((R)-2,2-dimethyl-1,3-dioxolan-4-yl)-2,2-dimethyltetrahydrofuro[2,3d][1,3]dioxol-6-yl 4-(( $N$-methylacetamido)methyl)benzoate (69)

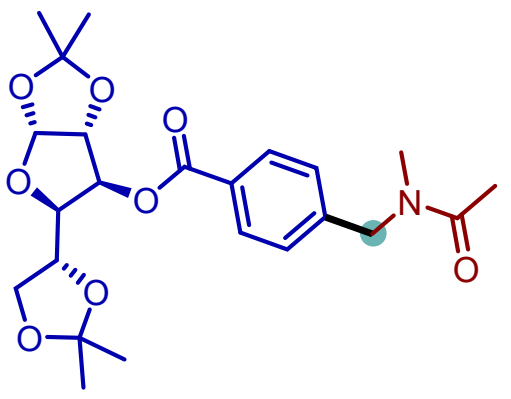

The compound was prepared according to the General Procedure $1 \quad(3 \mathrm{a} R, 5 R, 6 S, 6 \mathrm{a} R)-5-((R)-2,2-$ dimethyl-1,3dioxolan-4-yl)-2,2-dimethyltetrahydrofuro[2,3-d][1,3]dioxol6-yl 4-bromobenzoate ${ }^{14}(88.6 \mathrm{mg}, 0.2 \mathrm{mmol}), \mathrm{mpg}-\mathrm{CN}(10.0$ $\mathrm{mg}$ ), $\mathrm{NiBr}_{2} \bullet$ glyme (3.2 mg, 5 mol\%), 2,2'-bipyridine (1.6 mg 5 mol\%), $N, N$-dimethylacetamide $(1.0 \mathrm{~mL})$ and 2,6-lutidine $(70$ $\mu \mathrm{L} 0.6 \mathrm{mmol}$ ). The reaction mixture was irradiated under nitrogen atmosphere for $48 \mathrm{~h}$ using blue LED $(455( \pm 15) \mathrm{nm})$. The reaction mixture was then subjected to the work-up procedure as described in the General Procedure 1. Purification was performed using flash chromatography on silica gel using $\mathrm{PE} / \mathrm{EtOAc}$ solvent mixture as eluent to provide the title compound in $74 \%$ yield $(66.4 \mathrm{mg}) .{ }^{1} \mathrm{H}$ NMR $\left(300 \mathrm{MHz}, \mathrm{CDCl}_{3}\right) \delta 8.00(\mathrm{~d}, J=8.4 \mathrm{~Hz}) \& 7.95$ 
(d, $J=8.4 \mathrm{~Hz}, 2 \mathrm{H}$ combined), 7.29 (d, $J=8.4 \mathrm{~Hz}) \& 7.24$ (d, $J=8.4 \mathrm{~Hz}, 2 \mathrm{H}$ combined), 5.92 (d, $J=3.6 \mathrm{~Hz}, 0 \mathrm{H}), 5.91$ (d, $J=3.6 \mathrm{~Hz}, 1 \mathrm{H}$ combined), $5.48-5.43(\mathrm{~m}, 1 \mathrm{H}), 4.61(\mathrm{~s}) \& 4.57$ (s, 2H combined), 4.60(s) \& 4.58 ( $\mathrm{s}, 1 \mathrm{H}$ combined), $4.37-4.25(\mathrm{~m}, 2 \mathrm{H}), 4.13-3.99(\mathrm{~m}, 2 \mathrm{H}), 2.92$ (s, 3H), 2.15 (s) \& $2.10(\mathrm{~s}, 3 \mathrm{H}$ combined), $1.52(\mathrm{~s}, 3 \mathrm{H}), 1.38(\mathrm{~s}, 3 \mathrm{H}), 1.29(\mathrm{~s}, 3 \mathrm{H}), 1.23(\mathrm{~s}, 3 \mathrm{H})$. ${ }^{13} \mathrm{C}$ NMR (101 MHz, $\left.\mathrm{CDCl}_{3}\right) \delta 171.00 \& 170.9,164.9 \& 164.8,143.4 \& 142.6,130.4 \& 130.1$, $129.1 \& 128.6,127.9 \& 126.4,112.5 \& 112.4,109.4 \& 109.4,105.2,83.4,79.9,76.8 \& 76.6$, 72.6, $67.3 \& 67.2,54.1 \& 50.5,35.8 \& 33.9,26.8 \& 26.7,26.2 \& 25.2,21.7 \&$ 21.4. HRMS (ESI): $m / z$ calculated. for $\mathrm{C}_{23} \mathrm{H}_{32} \mathrm{~N}_{3} \mathrm{O}_{8}[\mathrm{M}+\mathrm{H}]^{+}:$450.2122; found 450.2115 .

$(2 R, 3 S, 4 S, 5 R, 6 S)-6-($ acetoxymethyl)-3-(4-( $(N-$

methylacetamido)methyl)benzamido)tetrahydro-2H-pyran-2,4,5-triyl triacetate (70)

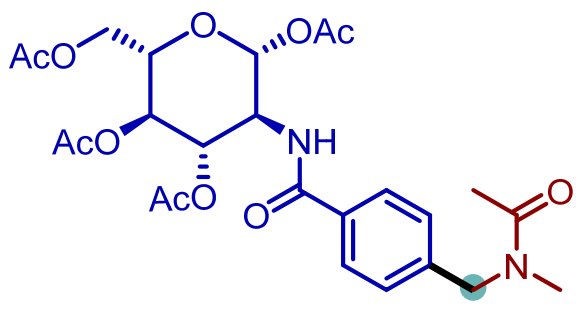

The compound was prepared according to the General

Procedure 1 (2R,3S,4S,5R,6S)-6-(acetoxymethyl)-3-(4bromobenzamido)tetrahydro-2H-pyran-2,4,5-triyl triacetate (106 mg, $0.2 \mathrm{mmol})$, mpg-CN (10.0 mg), $\mathrm{NiBr}{ }^{\bullet}$ glyme (3.2 mg, 5 mol\%), 2,2'-bipyridine (1.6 mg 5 mol\%), $N, N$-dimethylacetamide $(1.0 \mathrm{~mL})$ and 2,6-lutidine $(70 \mu \mathrm{L} 0.6 \mathrm{mmol})$. The reaction mixture was irradiated under nitrogen atmosphere for $72 \mathrm{~h}$ using blue LED (455 $( \pm 15) \mathrm{nm})$. The reaction mixture was then subjected to the work-up procedure as described in the General Procedure 1. Purification was performed using flash chromatography on silica gel using $\mathrm{PE} /$ EtOAc solvent mixture as eluent to provide the title compound in $74 \%$ yield $(79.3 \mathrm{mg}) .{ }^{1} \mathrm{H}$ NMR $\left(300 \mathrm{MHz}, \mathrm{CDCl}_{3}\right) \delta 7.76(\mathrm{~d}, J=8.3 \mathrm{~Hz}) \& 7.66(\mathrm{~d}, J=8.3 \mathrm{~Hz}, 2 \mathrm{H}$ combined), 7.49 (d, $J=9.5 \mathrm{~Hz}) \& 7.45(\mathrm{~d}, J=9.5 \mathrm{~Hz}, 1 \mathrm{H}$ combined), $7.18(\mathrm{~d}, J=8.3 \mathrm{~Hz}) \& 7.14(\mathrm{~d}, J=8.3 \mathrm{~Hz}$, 2H combined), 5.87 (d, $J=8.8 \mathrm{~Hz}, 1 \mathrm{H}), 5.39$ (ddd, $J=10.6,9.5,5.1 \mathrm{~Hz}, 1 \mathrm{H}), 5.15$ (t, $J=9.5$ $\mathrm{Hz}, 1 \mathrm{H}), 4.65-4.46(\mathrm{~m}, 3 \mathrm{H}), 4.28(\mathrm{~d}, J=4.8 \mathrm{~Hz}) \& 4.24$ (d, J=4.8 Hz, 1H combined), 4.14 (d, $J=2.0 \mathrm{~Hz}) \& 4.09(\mathrm{~d}, J=2.0 \mathrm{~Hz}, 1 \mathrm{H}$ combined), $3.89-3.74(\mathrm{~m}, 1 \mathrm{H}), 2.90$ (s) \& 2.89 (s, 3H combined), 2.15 (s, 2H), 2.08 (s) \& 2.07 (s, 3H combined), 2.02 (s) \& 2.01 (s, 3H combined), 1.94 (s) \& 1.93 (s, 3H combined). ${ }^{13} \mathrm{C}$ NMR $\left(75 \mathrm{MHz}, \mathrm{CDCl}_{3}\right) \delta 171.3,171.1 \& 170.8,169.5$ \& 169.4, 167.2 \& 166.8, $141.3 \& 140.5,133.3 \& 133.1,128.0 \& 127.6,126.25,92.7,72.8$, 68.2, 61.8, $53.9 \& 50.4,53.2 \& 53.12,35.8 \& 34.0,21.8 \& 21.4,20.9 \& 20.8,20.7 \& 20.6$. HRMS (ESI): $m / z$ calculated. for $\mathrm{C}_{25} \mathrm{H}_{33} \mathrm{~N}_{2} \mathrm{O}_{11}[\mathrm{M}+\mathrm{H}]^{+}:$537.2079; found 537.2090. 


\section{$N$-(4'-chloro-[1,1'-biphenyl]-2-yl)-2-(( $N$-methylacetamido)methyl)nicotinamide (71)}

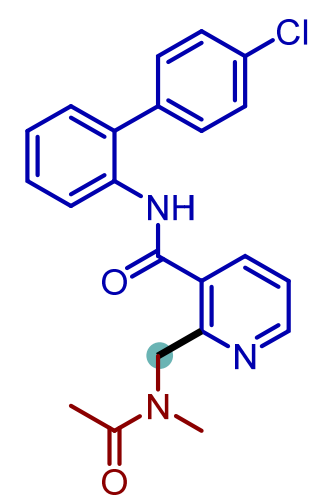

The compound was prepared according to the General Procedure 2 using boscalid (68.6 mg, $0.2 \mathrm{mmol}), \mathrm{mpg}-\mathrm{CN}$ (10.0 mg), $\mathrm{NiCl}_{2} \bullet$ glyme (2.2 mg, $5 \mathrm{~mol} \%$ ), 2,2'-bipyridine (1.6 mg $5 \mathrm{~mol} \%$ ), $N, N$-dimethylacetamide $(1.0 \mathrm{~mL})$ and 2,6-lutidine $(70 \mu \mathrm{L} 0.6 \mathrm{mmol})$. The reaction mixture was irradiated under nitrogen atmosphere for 72h using blue LED (455 $( \pm 15)$ $\mathrm{nm})$. The reaction mixture was then subjected to the work-up procedure as described in the General Procedure 2. Purification was performed using flash chromatography on silica gel using $\mathrm{PE} / \mathrm{EtOAc}_{\mathrm{E}} \mathrm{EN}$ solvent mixture as eluent to provide the title compound in $61 \%$ yield $(47.9 \mathrm{mg}) .{ }^{1} \mathrm{H}$ NMR (400 MHz, $\left.\mathrm{CDCl}_{3}\right) \delta 10.25(\mathrm{~s}, 1 \mathrm{H}), 8.54$ (d, $J=3.8 \mathrm{~Hz}) \& 8.45(\mathrm{~d}, J=3.8 \mathrm{~Hz}, 1 \mathrm{H}$ combined), 7.77 (d, $J=7.9 \mathrm{~Hz}, 1 \mathrm{H}), 7.72$ (d, $J=7.9$ $\mathrm{Hz}, 1 \mathrm{H}), 7.41-7.29$ (m, 2H), $7.24-7.04$ (m, 6H), 4.72 (s), 4.19 (s, 2H combined), 3.26 (s) \& $2.76\left(\mathrm{~s}, 3 \mathrm{H}\right.$ combined), $2.01(\mathrm{~s}) \& 1.87\left(\mathrm{~s}, 3 \mathrm{H}\right.$ combined). ${ }^{13} \mathrm{C} \mathrm{NMR}\left(101 \mathrm{MHz}, \mathrm{CDCl}_{3}\right) \delta 171.5$ \& 171.3, $167.2 \& 167.0,155.6 \& 153.6,151.2 \& 150.6,139.7 \& 138.3,137.4,135.8,134.9$, $134.2 \& 133.4,132.1 \& 130.9,130.6 \& 130.4,129.4 \& 128.4,129.1 \& 128.62,126.1 \& 126.0$, $125.9 \& 125.8,122.6 \& 122.4,53.8 \& 52.7,40.5 \& 34.1,22.4 \& 21.7$. HRMS (ESI): $\mathrm{m} / \mathrm{z}$ calculated. for $\mathrm{C}_{22} \mathrm{H}_{21} \mathrm{ClN}_{3} \mathrm{O}_{2}[\mathrm{M}+\mathrm{H}]^{+}: 394.1317$; found 394.1321 .

\section{$N$-(adamantan-1-yl)-4-(( $N$-methylacetamido)methyl)benzamide (72)}

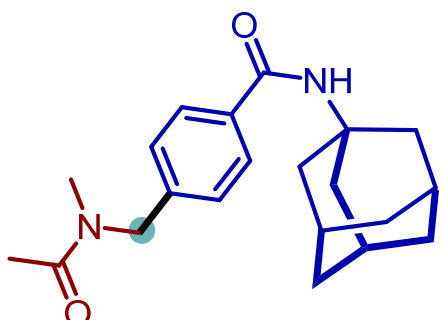

The compound was prepared according to the General Procedure $1 \mathrm{~N}$-(adamantan-1-yl)-4-bromobenzamide $(66.8 \mathrm{mg}$, $0.2 \mathrm{mmol})$, mpg-CN (10.0 mg), NiBr2•glyme (3.2 mg, $5 \mathrm{~mol} \%$ ), 2,2'-bipyridine (1.6 mg $5 \mathrm{~mol} \%), N, N$-dimethylacetamide $(1.0 \mathrm{~mL})$ and 2,6-lutidine (70 $\mu \mathrm{L} 0.6 \mathrm{mmol})$. The reaction mixture was irradiated under nitrogen atmosphere for $72 \mathrm{~h}$ using blue LED $(455( \pm 15) \mathrm{nm})$. The reaction mixture was then subjected to the work-up procedure as described in the General Procedure 1. Purification was performed using flash chromatography on silica gel using PE/EtOAc solvent mixture as eluent to provide the title compound in $64 \%$ yield $(43.5 \mathrm{mg}) .{ }^{1} \mathrm{H} \mathrm{NMR}(400 \mathrm{MHz}$, $\left.\mathrm{CDCl}_{3}\right) \delta 7.69(\mathrm{~d}, J=8.3 \mathrm{~Hz}) \& 7.63(\mathrm{~d}, J=8.3 \mathrm{~Hz}, 2 \mathrm{H}$ combined $), 7.22(\mathrm{~d}, J=8.3 \mathrm{~Hz}) \& 7.15$ (d, $J=8.3 \mathrm{~Hz}, 2 \mathrm{H}$ combined), 5.90 (s) \& 5.88 (s, $1 \mathrm{H}$ combined), 4.55 (s) \& 4.51 (s, 2H combined), 2.88 (s) \& 2.87 (s, 3H combined), 2.11 (s) \& 2.08 (s, 12H combined), 1.67 (s, 6H). ${ }^{13} \mathrm{C} \mathrm{NMR}\left(101 \mathrm{MHz}, \mathrm{CDCl}_{3}\right) \delta 170.9 \& 170.8,166.3 \& 166.0,140.5 \& 139.6,135.5 \& 135.2$, $127.9 \& 127.5,127.1 \& 126.3,53.4 \& 50.3,52.4 \& 52.3$, , 41.6, 36.4, $35.6 \& 33.8,29.5,21.7$ \& 21.4. HRMS (ESI): $\mathrm{m} / \mathrm{z}$ calculated. for $\mathrm{C}_{21} \mathrm{H}_{29} \mathrm{~N}_{2} \mathrm{O}_{2}[\mathrm{M}+\mathrm{H}]^{+}:$341.2224; found 341.2231. 
yl)acetate (73)

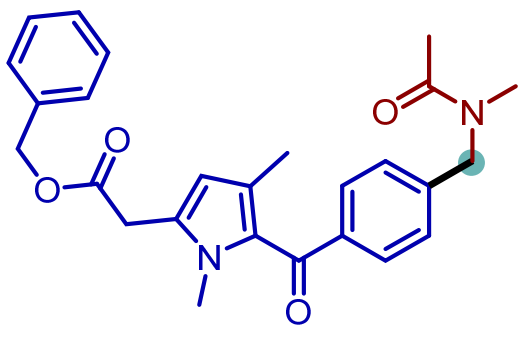

The compound was prepared according to the General Procedure 2 using benzyl ester of zomepirac ${ }^{15}(69.4 \mathrm{mg}, 0.2$ mmol), mpg-CN (10.0 mg), $\mathrm{NiCl}_{2} \bullet$ glyme (2.2 mg, $5 \mathrm{~mol} \%$ ), 2,2'-bipyridine (1.6 mg $5 \mathrm{~mol} \%$ ), $N, N$-dimethylacetamide (1.0 $\mathrm{mL})$ and 2,6-lutidine ( $70 \mu \mathrm{L} 0.6 \mathrm{mmol})$. The reaction mixture was irradiated under nitrogen atmosphere for $72 \mathrm{~h}$ using blue LED $(455( \pm 15) \mathrm{nm})$. The reaction mixture was then subjected to the work-up procedure as described in the General Procedure 2. Purification was performed using flash chromatography on silica gel using $\mathrm{PE} / \mathrm{EtOAc}_{\mathrm{E}} \mathrm{Et}_{3} \mathrm{~N}$ solvent mixture as eluent to provide the title compound in 58\% yield (50.1 mg). ${ }^{1} \mathrm{H}$ NMR (400 $\left.\mathrm{MHz}, \mathrm{CDCl}_{3}\right) \delta 7.62(\mathrm{~d}, J=8.2 \mathrm{~Hz}) \& 7.57(\mathrm{~d}, J=8.2 \mathrm{~Hz}, 2 \mathrm{H}$ combined), $7.32-7.12(\mathrm{~m}, 7 \mathrm{H})$, $5.85(\mathrm{~s}) \& 5.84$ (s, $1 \mathrm{H}$ combined), 5.09 (s, 2H), 4.56 (s) \& 4.50 (s, 2H combined), 3.62 (s) \& 3.61 (s, 5H combined), 2.87 (s) \& 2.84 (s, 3H combined), 2.09 (s) \& 2.06 (s, 3H combined), $1.64(\mathrm{~s}) \& 1.62$ (s, $3 \mathrm{H}$ combined). ${ }^{13} \mathrm{C} \mathrm{NMR}\left(101 \mathrm{MHz}, \mathrm{CDCl}_{3}\right) \delta 187.6 \& 187.2,171.1 \& 170.9$, $169.5 \& 169.4,141.35 \& 140.6,140.4 \& 140.2,135.6 \& 135.5,132.9 \& 132.6,130.1 \& 130.0$, $129.9 \& 129.6,129.1 \& 129.0,128.7 \& 128.4,128.61,127.9,126.4,112.6 \& 112.5,67.3 \&$ 67.2, $54.2 \& 50.5,35.7 \& 33.9,33.2,32.92,21.9 \& 21.6,14.5 \& 14.4$. HRMS (ESI): $m / z$ calculated. for $\mathrm{C}_{26} \mathrm{H}_{29} \mathrm{~N}_{2} \mathrm{O}_{4}[\mathrm{M}+\mathrm{H}]^{+}: 433.2122$; found 433.2128 .

\section{$N$-methyl- $N$-(4-(1-(4-sulfamoylphenyl)-3-(trifluoromethyl)-1H-pyrazol-5-} yl)benzyl)acetamide (74)

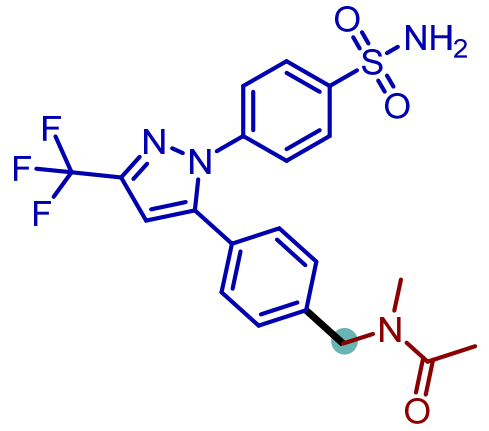

The compound was prepared according to the General Procedure $1 \mathrm{Br}$-celecoxib (89.2 mg, $0.2 \mathrm{mmol})$, mpg-CN (10.0 $\mathrm{mg}$ ), $\mathrm{NiBr}_{2} \bullet$ glyme (3.2 mg, $5 \mathrm{~mol} \%$ ), 2,2'-bipyridine (1.6 mg 5 mol\%), $N, N$-dimethylacetamide $(1.0 \mathrm{~mL})$ and 2,6-lutidine $(70$ $\mu \mathrm{L} \quad 0.6 \mathrm{mmol})$. The reaction mixture was irradiated under nitrogen atmosphere for $72 \mathrm{~h}$ using blue LED $(455( \pm 15) \mathrm{nm})$. The reaction mixture was then subjected to the work-up procedure as described in the General Procedure 1. Purification was performed using flash chromatography on silica gel using PE/EtOAc solvent mixture as eluent to provide the title compound in 77\% yield (69.6 mg). ${ }^{1} \mathrm{H}$ NMR (400 MHz, $\left.\mathrm{CDCl}_{3}\right) \delta 7.91-7.78(\mathrm{~m}, 2 \mathrm{H}), 7.41-$ $7.33(\mathrm{~m}, 2 \mathrm{H}), 7.24-7.11(\mathrm{~m}, 4 \mathrm{H}), 6.76(\mathrm{~s}) \& 6.73(\mathrm{~s}, 1 \mathrm{H}$ combined), $5.71(\mathrm{~s}, 2 \mathrm{H}), 4.55(\mathrm{~s}) \&$ $4.53\left(\mathrm{~s}, 2 \mathrm{H}\right.$ combined), 2.96 (s) \& 2.90 (s, 3H combined), 2.14 (s) \& 2.11 (s, 3H combined). ${ }^{13} \mathrm{C}$ 
NMR $\left(101 \mathrm{MHz}, \mathrm{CDCl}_{3}\right) \delta 171.4,144.8 \& 144.5,144.4 \& 144.3,144.0 \& 143.9,142.3 \& 142.2$, $138.9 \& 138.3,129.5 \& 129.2,128.3 \& 128.1,127.7 \& 127.6,127.5 \& 127.1,125.6 \& 125.5$, $123.8(\mathrm{q}, J=269.2 \mathrm{~Hz}) \& 122.4(\mathrm{q}, J=269.2 \mathrm{~Hz}), 106.7 \& 106.5,54.0 \& 50.6,36.2 \& 34.1$, $21.8 \&$ 21.5. HRMS (ESI): $\mathrm{m} / \mathrm{z}$ calculated. for $\mathrm{C}_{20} \mathrm{H}_{20} \mathrm{~F}_{3} \mathrm{~N}_{4} \mathrm{O}_{3} \mathrm{~S}[\mathrm{M}+\mathrm{H}]^{+}:$453.1203; found 453.1212 . 


\section{Characterization of recovered mpg-CN photocatalyst}

After the photocatalytic tests, mpg-CN was washed with a series of solvents and $1 \mathrm{M} \mathrm{HCl}$ solution to prepare the catalyst for post characterization. The material was characterized by the same set of techniques used for fresh mpg-CN. Position and intensity of all peaks observed in FT-IR spectrum of mpg-CN revealed that bulk chemical structure of the photocatalyst has not changed (Figure S14). EDX and XPS revealed enhanced oxygen content on the surface of the photocatalyst (Table S7, S8 and Figure S15b). Additionally, ICP-OES revealed that mpg-CN after the photocatalytic tests contained $0.0709 \pm 0.0131 \mathrm{wt}$. \% of Ni (Table S9, entry 2). Detection limit of Ni by ICP-OES was determined to be $>0.0013 \pm 0.00001$ wt. \% (Table S9, entry 1). However, Ni 2p XPS did not show a distinct signal of nickel (Figure S24b). Given that XPS is surface-sensitive technique, such result confirmed that Ni in the sample is located mainly in the bulk of the material and it was completely removed from the surface upon washing with $\mathrm{HCl}$ acid solution. Similarly, no signal was observed in $\mathrm{Br} 3 \mathrm{~d}$ XPS (Figure S25b), which potentially could be related to using $\mathrm{NiBr}_{2}$ as metal precursor. HR-TEM of the recovered mpg$\mathrm{CN}$ revealed that mesoporous structure has retained (Figure S23d). In HR-TEM images we observed dark spots with diameter of 2-6 $\mathrm{nm}$ that could be ascribed to $\mathrm{Ni}(0)$ nanoparticles. Indeed, deposition of $\mathrm{Ni}(0)$ nanoparticles, albeit significantly larger amount (1.4-12.6 wt.\%) of Ni black, compared to $\sim 0.071$ wt.\% in this work, has been reported earlier by Pieber et al. in dual $\mathrm{Ni} /$ carbon nitride photocatalysis in $\mathrm{C}-\mathrm{N}$ coupling. ${ }^{16,17}$ Despite reaction conditions in earlier reports and herein, strictly speaking, are different, they could not account completely for 20 to 180 times larger difference in Ni content in recovered photocatalysts. The reason for such discrepancy, in our opinion, lies mainly in different chemical structures of carbon nitride photocatalysts. Thus, in the present work mpg-CN belongs to covalent carbon nitrides, while carbon nitride used by Pieber et al., CN-OA-m ${ }^{18}-$ to ionic carbon nitrides. ${ }^{19}$ Due to abundant nitrogen atoms, carbon nitrides can serve as polydentate ligands to coordinate Ni species. However, taking into account differences in their structures, purely covalent structure (in mpg$\mathrm{CN}$ ) and ionic represented by $\mathrm{N}-\mathrm{K}$ bonds (in $\mathrm{CN}-\mathrm{OA}-\mathrm{m}$ ), the coordination mode of $\mathrm{NiBr}_{2}$ is different as shown in Figure S26. In covalent carbon nitrides, such as mpg-CN, Ni precursor coordinated by the carbon nitride scaffold and gives 16e Ni(II) chelate complex (Figure S26a). In ionic carbon nitrides, such as CN-OA-m, more reactive $14 \mathrm{e} \mathrm{Ni(II)-amide} \mathrm{complex} \mathrm{is} \mathrm{formed}$ (Figure S26b). Our results demonstrate that the problem of Ni black formation in dual $\mathrm{Ni} /$ photoredox catalysis, in addition to adjusting rates of oxidative addition and reductive elimination by tuning energy of incident light and concentration of reagents, ${ }^{17}$ could be also eliminated by selecting robust carbon nitride photocatalyst able to stabilize Ni species and does 
not compromise rate of the entire process. Modification of mpg-CN surface also results in slight shift of absorption onset in DRUV-vis spectrum (Figure S20a) and expansion of optical band gap by $\sim 0.05 \mathrm{eV}$ (Figure S20b). In steady-state PL such surface modification of mpg-CN is observed as blue shift of fluorescence by $\sim 0.1 \mathrm{eV}$ (Figure S21). Morphology of recovered mpg-CN particles adopted rounded shape compared to more rough surface of fresh mpg-CN (Figure S22b). Overall, post characterization of mpg-CN clearly shows robustness and stability of this photocatalyst.

Table S7. EDX elemental analysis.

\begin{tabular}{|l|l|l|l|}
\hline Sample & C wt. \% & N wt. \% & O wt. \% \\
\hline Fresh mpg-CN & $36 \pm 1.3$ & $62 \pm 1.2$ & $2 \pm 0.4$ \\
\hline Recovered mpg-CN & $35 \pm 0.6$ & $61 \pm 0.7$ & $4 \pm 0.3$ \\
\hline
\end{tabular}

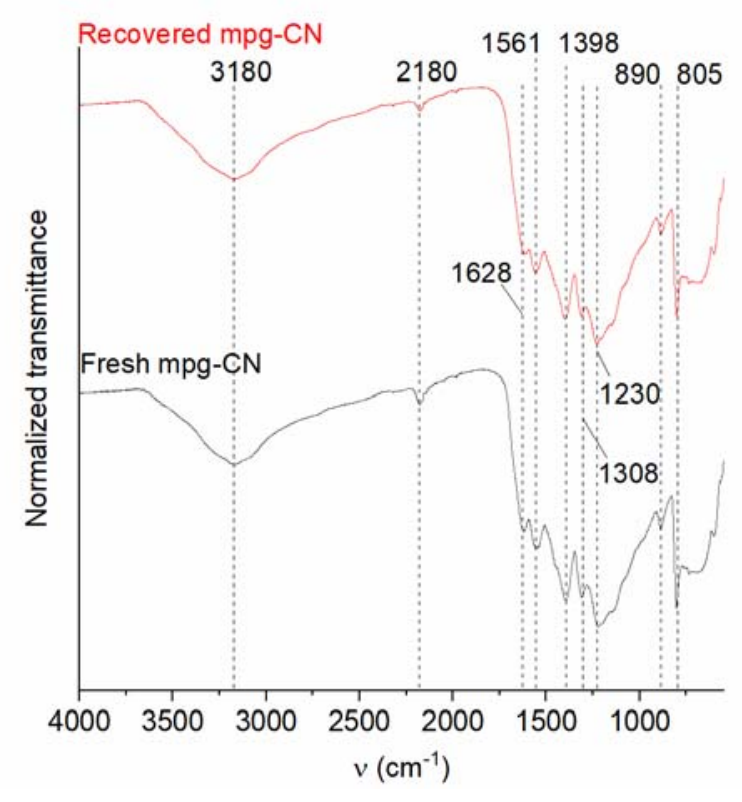

Figure S14. FT-IR spectra of fresh and recovered mpg-CN.

Table S8. XPS elemental analysis.

\begin{tabular}{|c|c|c|c|}
\hline Sample & C at. $\%$ & N at. $\%$ & O at. $\%$ \\
\hline Fresh mpg-CN & 29.1 & 64.8 & 6.1 \\
\hline Recovered mpg-CN & 30.7 & 70.2 & 9.6 \\
\hline
\end{tabular}


a)

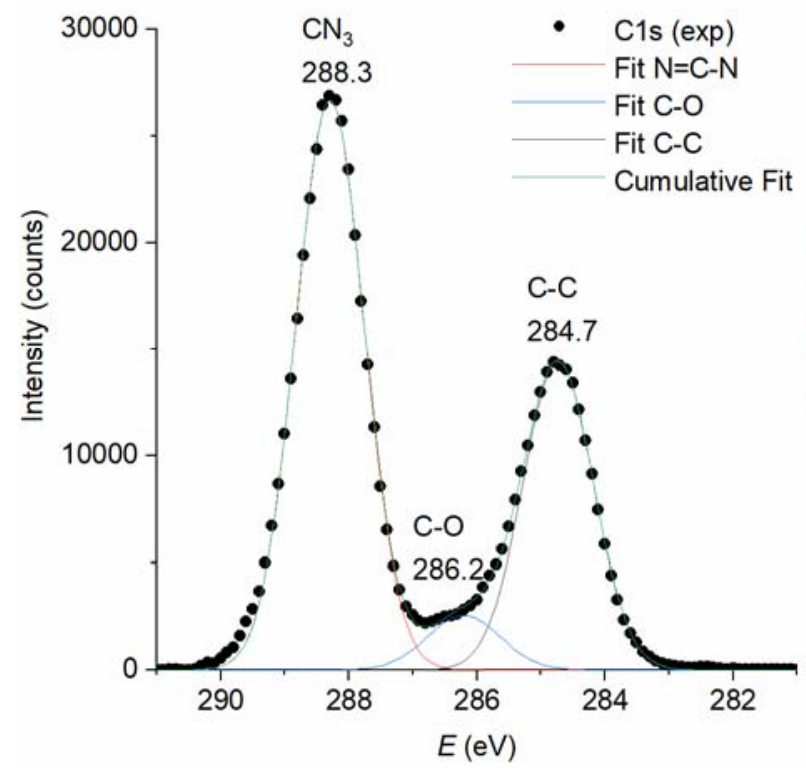

b)

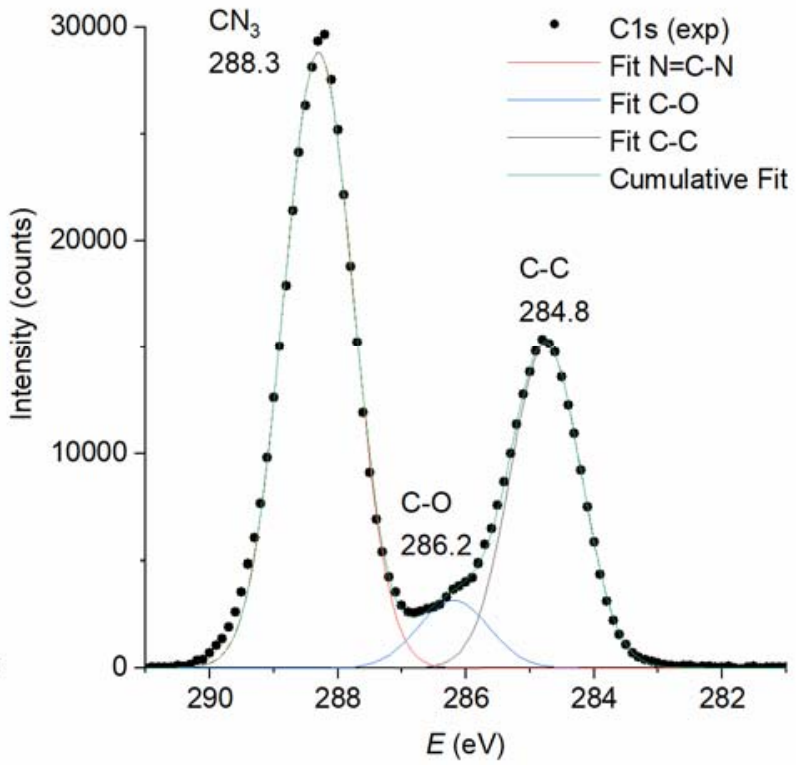

Figure S15. C 1s XPS of (a) fresh mpg-CN. (b) recovered mpg-CN.

a)

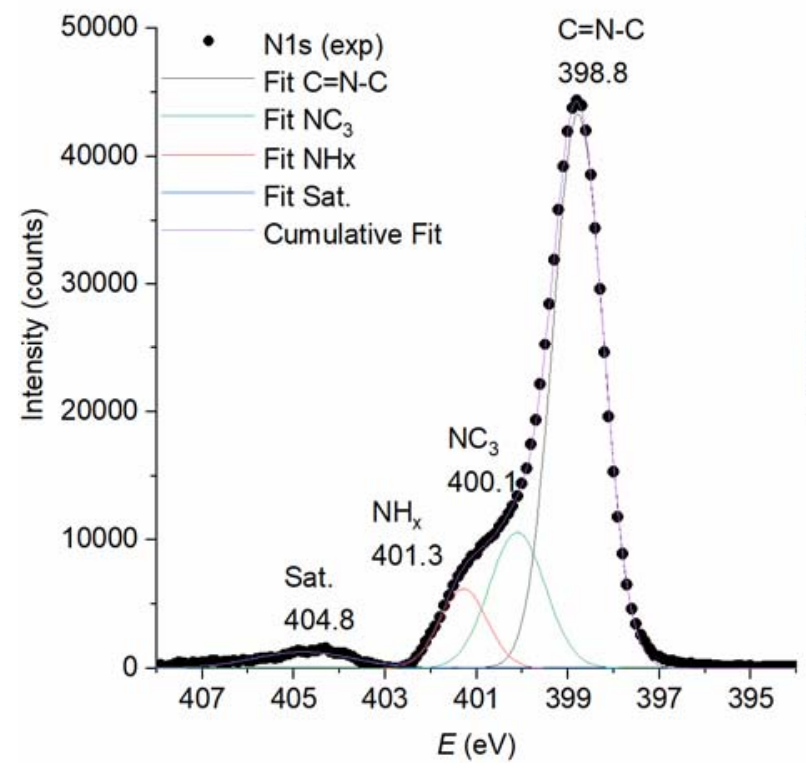

b)

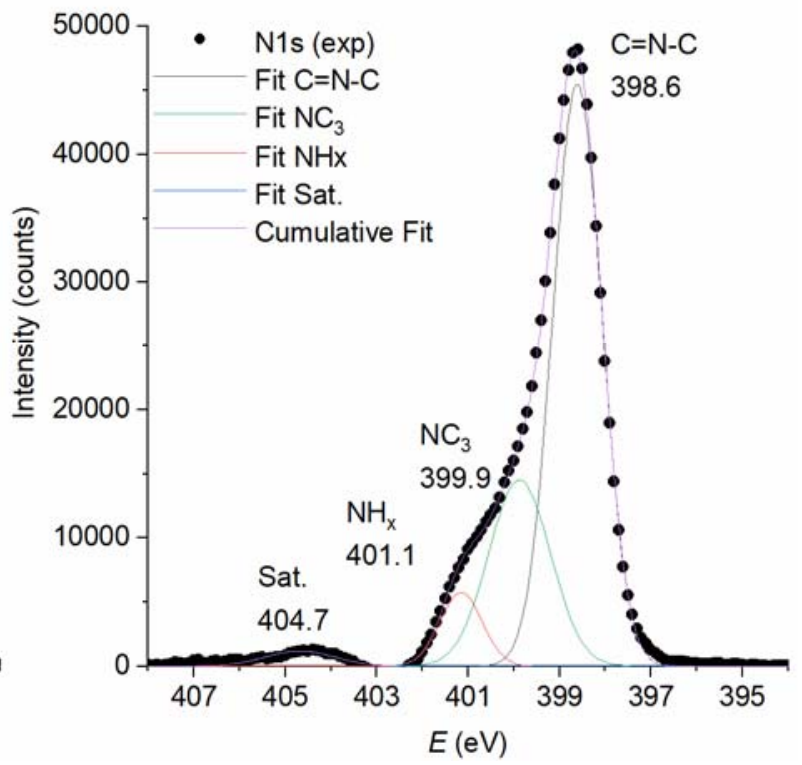

Figure S16. N 1s XPS of (a) fresh mpg-CN. (b) recovered mpg-CN. 
a)

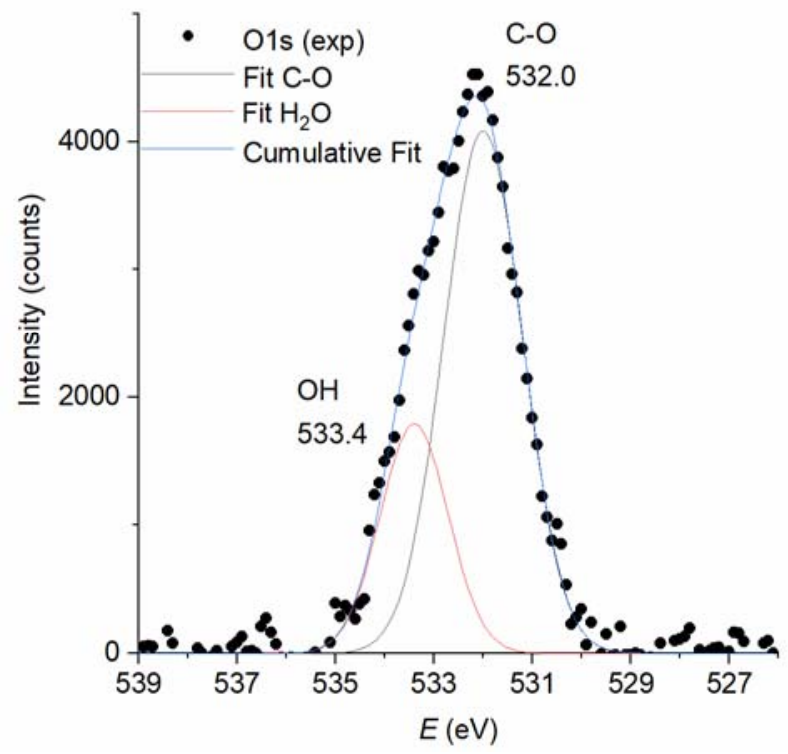

b)

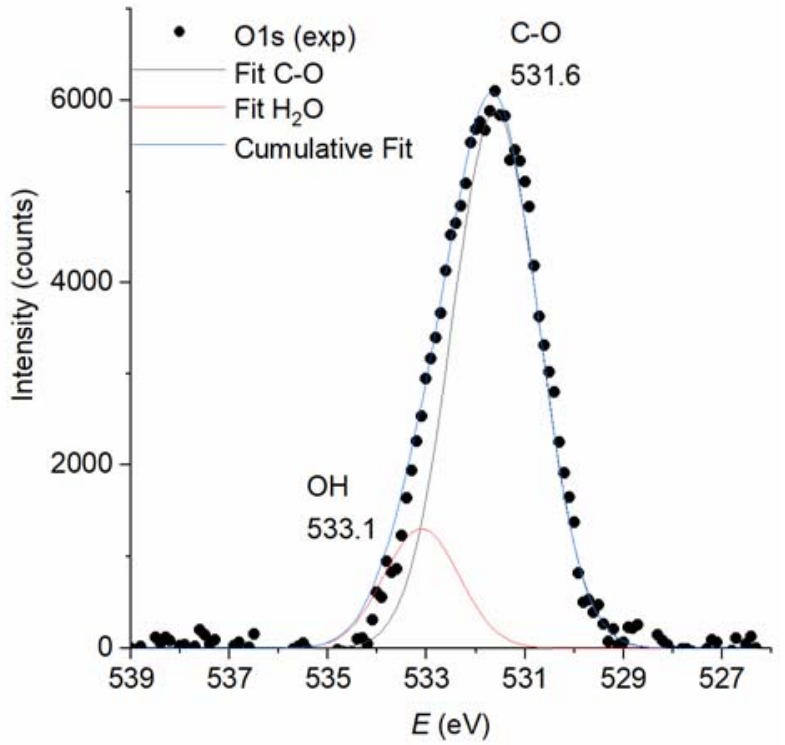

Figure S17. O 1s XPS of (a) fresh mpg-CN. (b) recovered mpg-CN.

a)

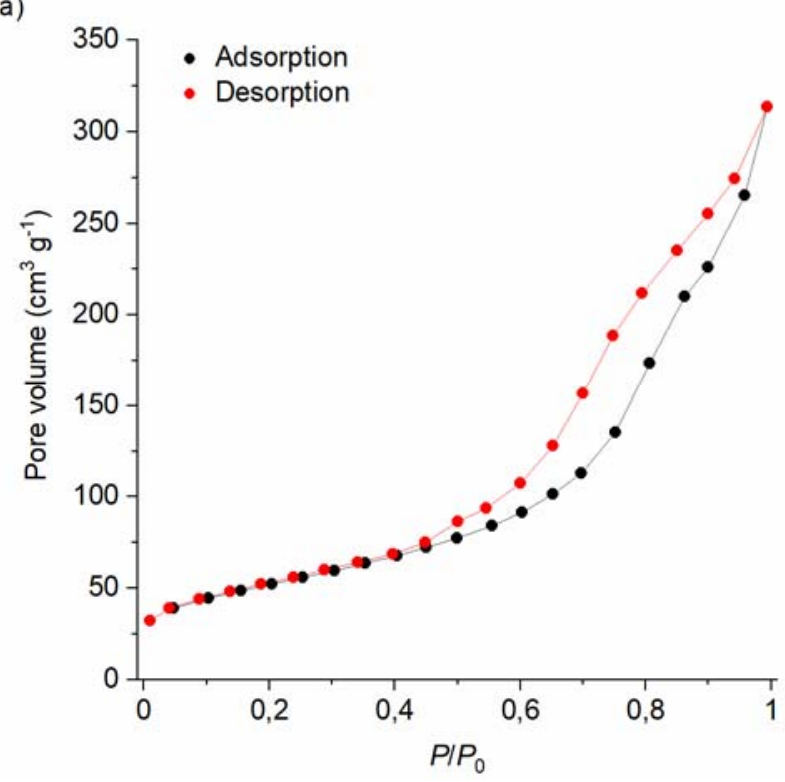

b)

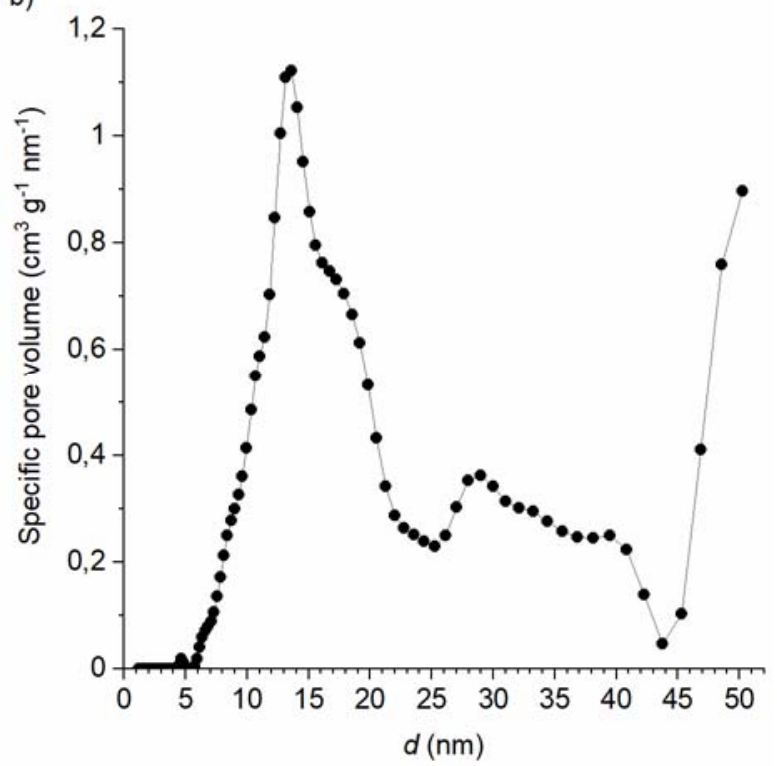

Figure S18. (a) $\mathrm{N}_{2}$ sorption isotherm recorded at $77 \mathrm{~K}$. (b) Pore size distribution in mpg-CN. 


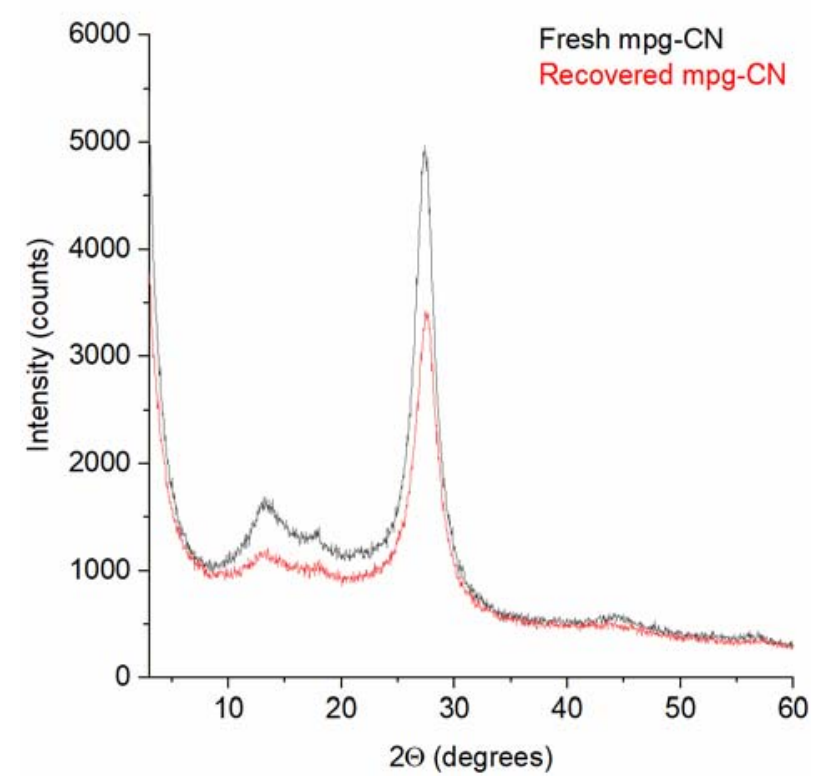

Figure S19. PXRD pattern of fresh and recovered mpg-CN.

a)

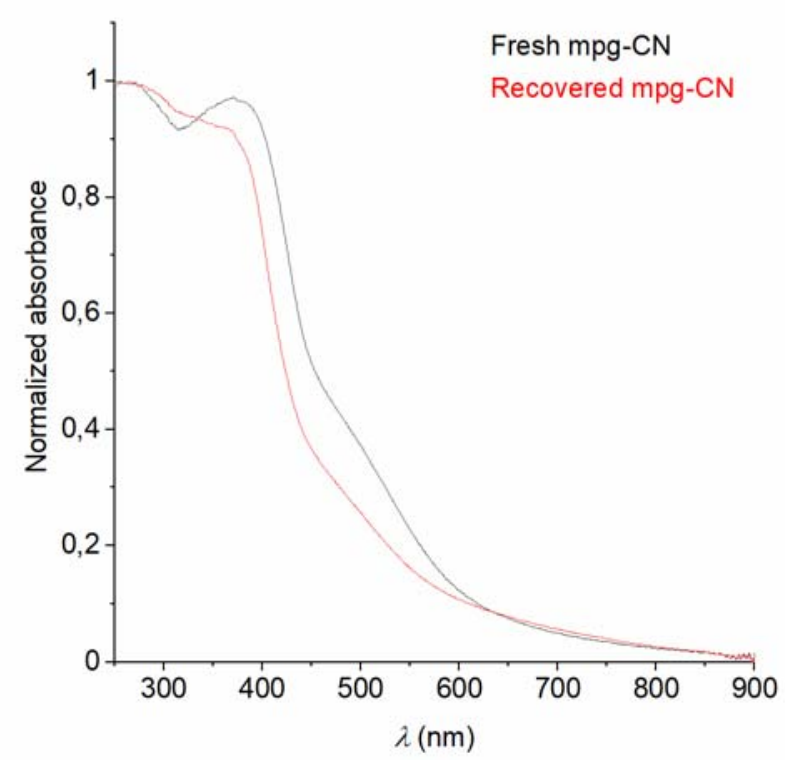

b)

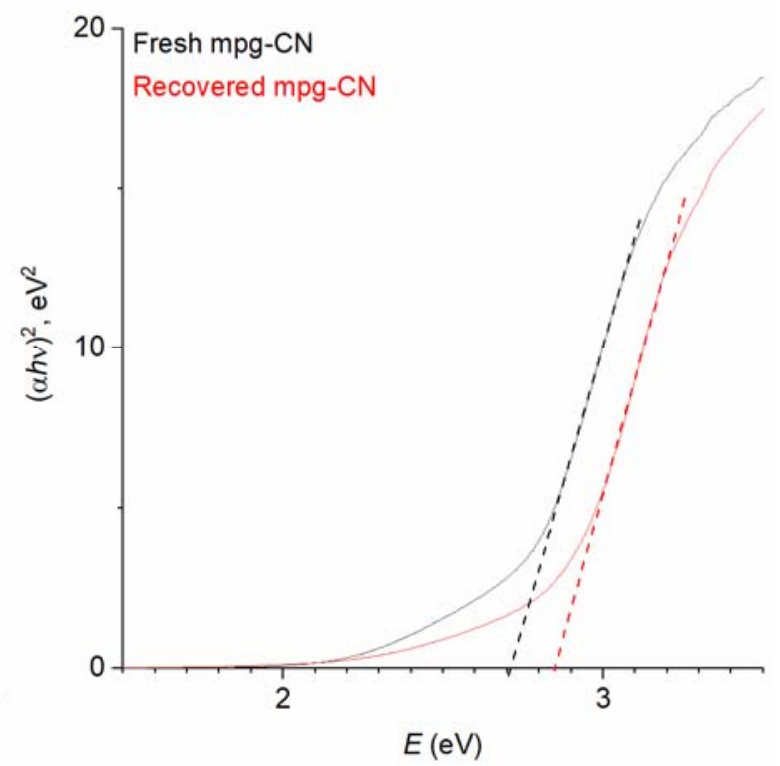

Figure S20. (a) DRUV-vis absorption spectra. (b) Tauc plots of fresh and recovered mpg-CN 


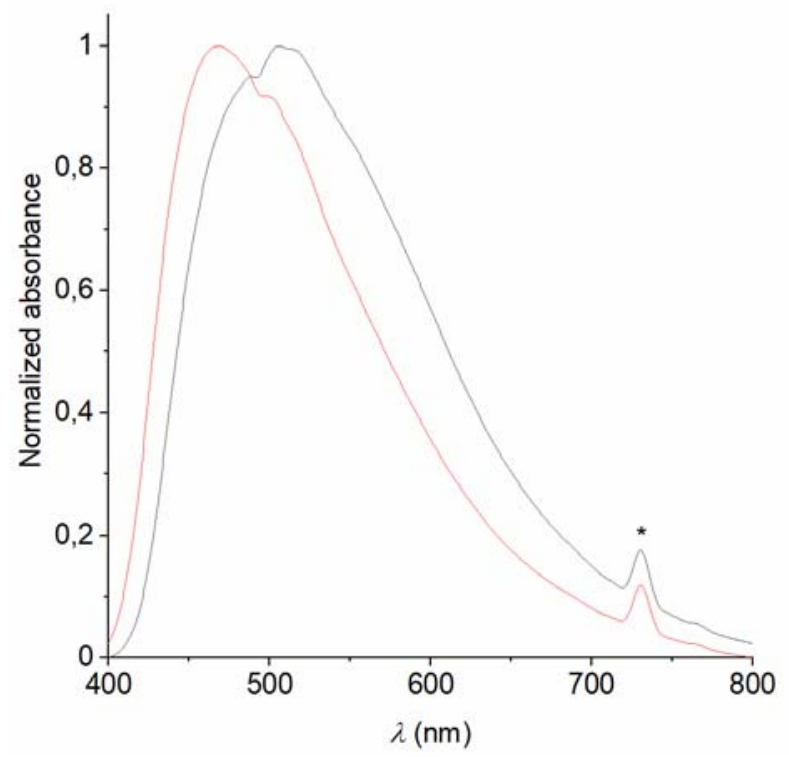

Figure S21. Steady state PL of fresh mpg-CN and recovered after the photocatalytic experiment. Samples were excited with $\lambda=365 \mathrm{~nm}$. Asterisk denotes $2^{\text {nd }}$ order excitation light diffraction.

a)

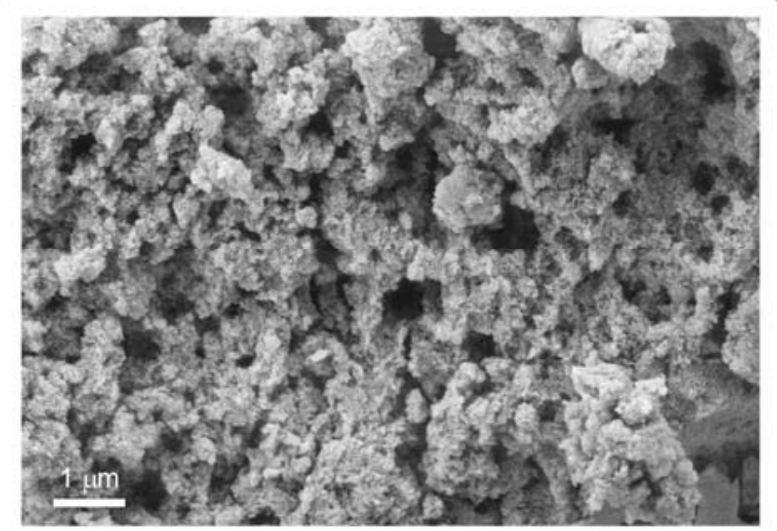

b)

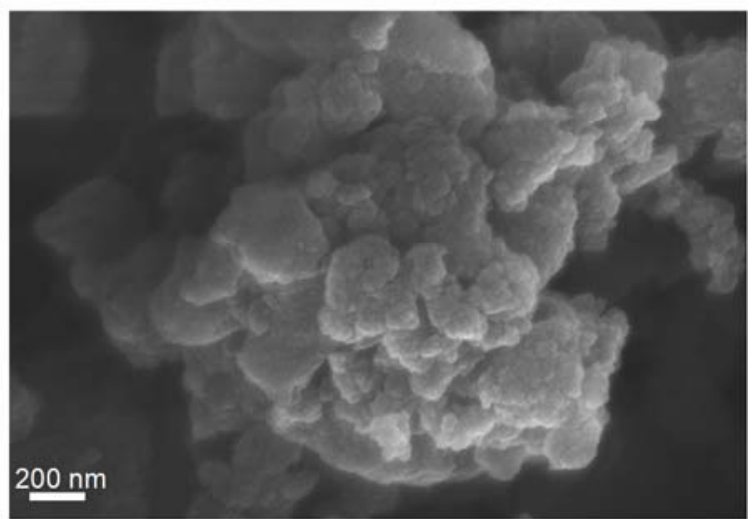

Figure S22. SEM (a) fresh mpg-CN. (b) recovered mpg-CN. 


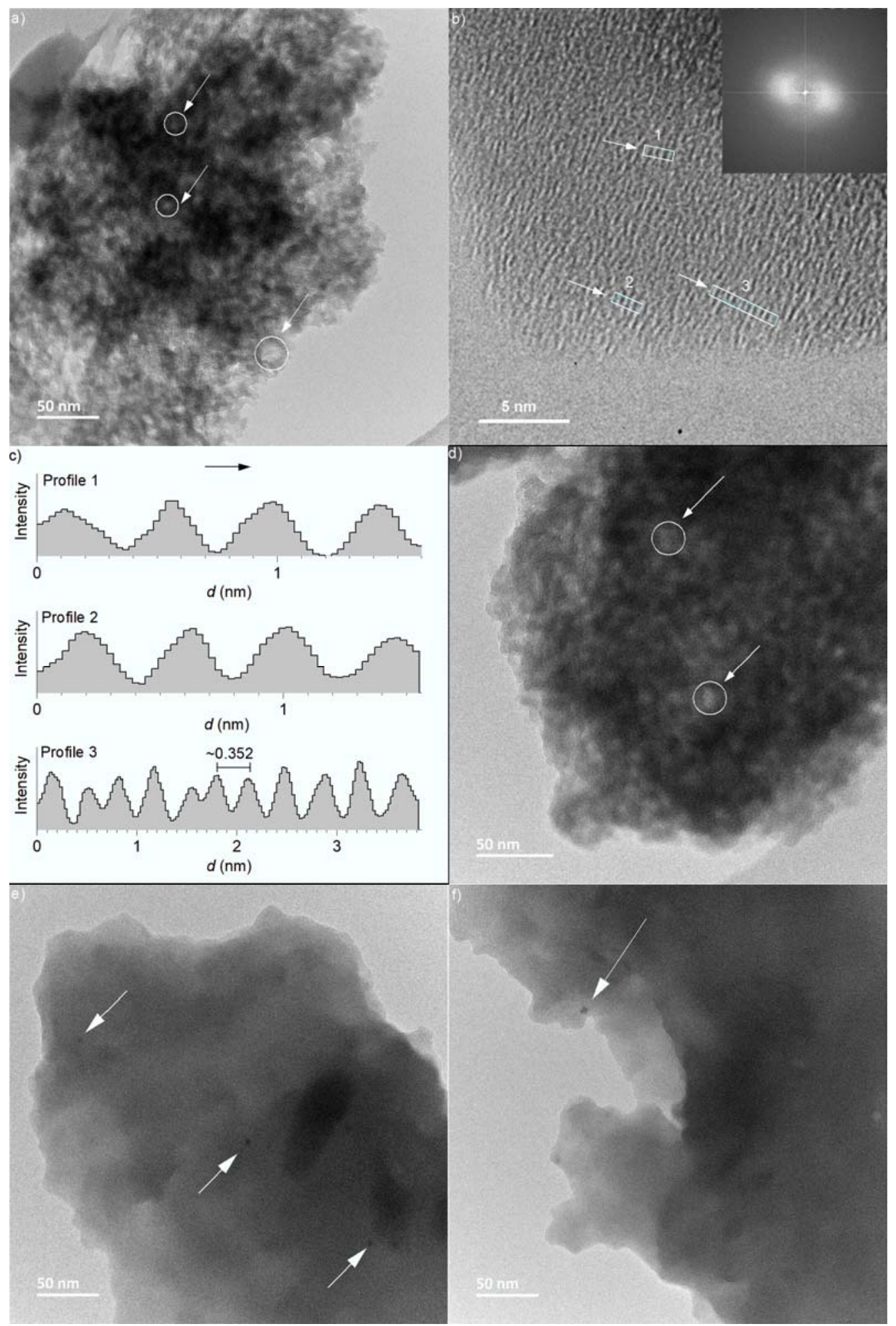

Figure S23. (a) overview TEM image of fresh mpg-CN, circles mark mesopores. (b) HR-TEM image of fresh mpg-CN with FFT in inset. (c) Profile analysis of the corresponding areas marked in the panel. (d) Overview TEM image of recovered mpg-CN. (e, f) TEM images of recovered mpg-CN. Arrows mark $\mathrm{Ni}(0)$ nanoparticles. 
Table S9. ICP-OES elemental analysis.

\begin{tabular}{|c|c|}
\hline Sample & Ni wt. $\%$ \\
\hline Fresh mpg-CN & $0.0013 \pm 0.00001$ \\
\hline Recovered mpg-CN & $0.0709 \pm 0.0131$ \\
\hline
\end{tabular}

a)

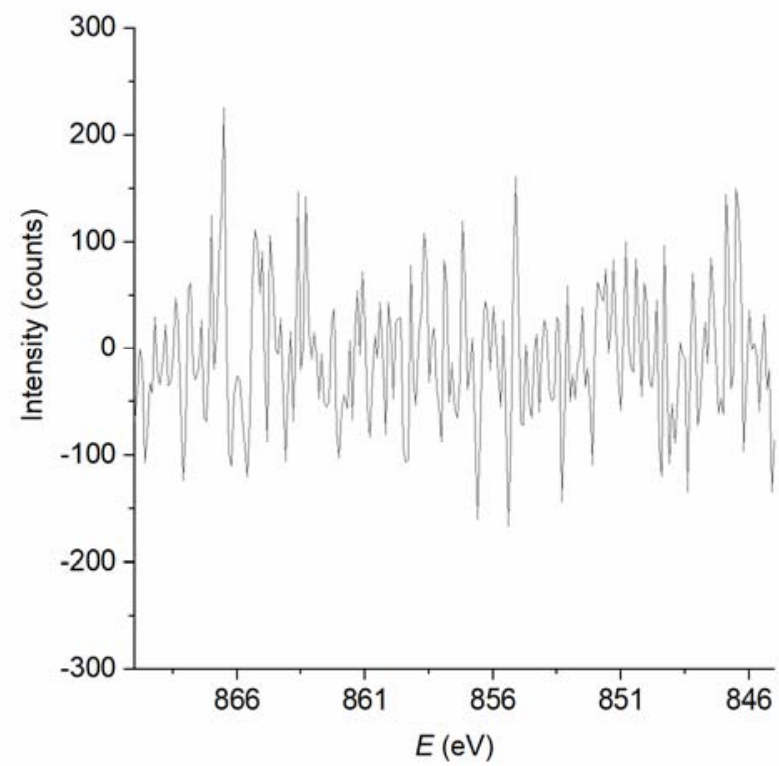

b)

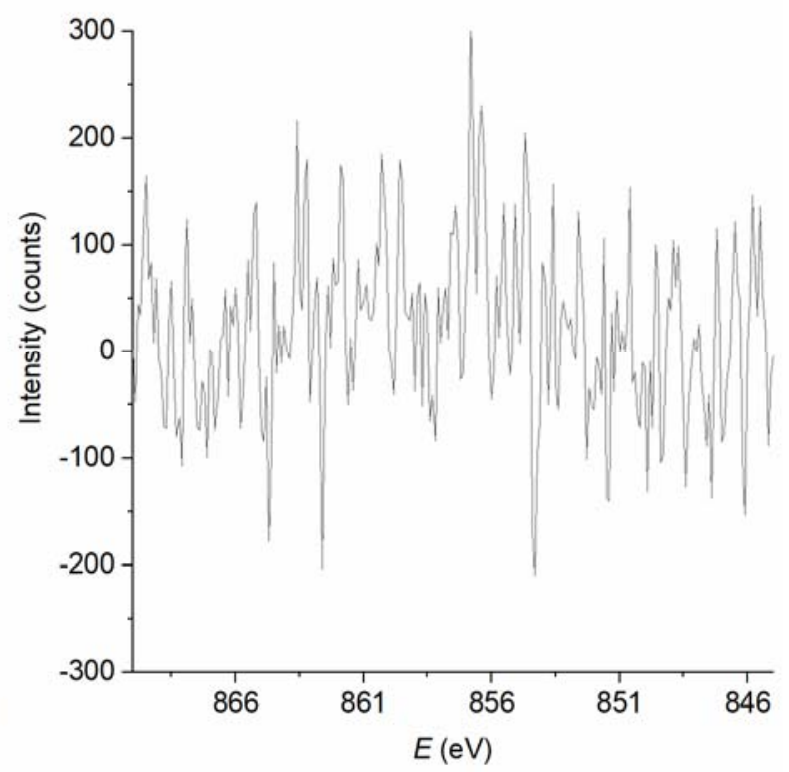

Figure S24. Ni 2p XPS of (a) fresh mpg-CN. (b) recovered mpg-CN.

a)

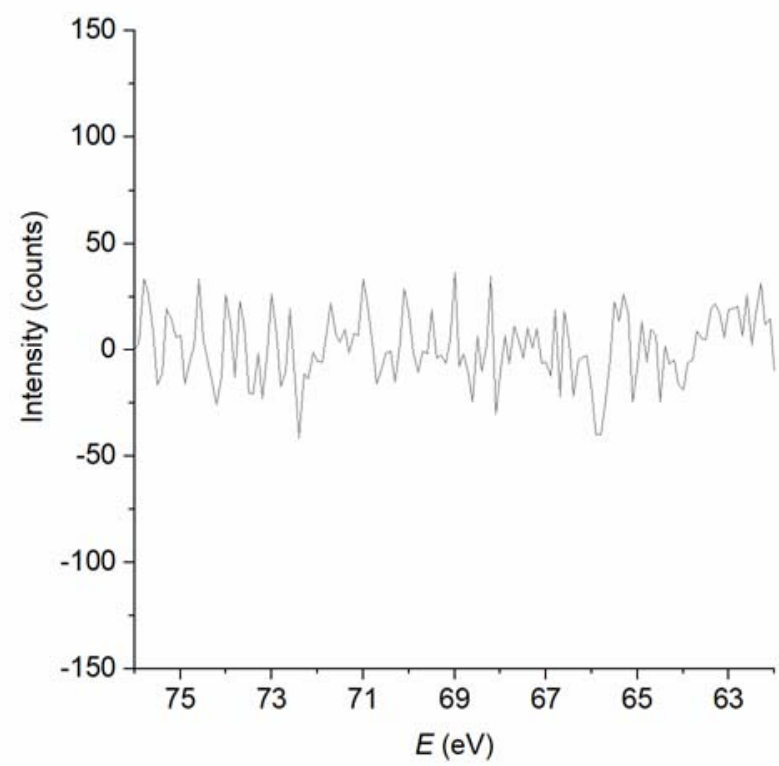

b)

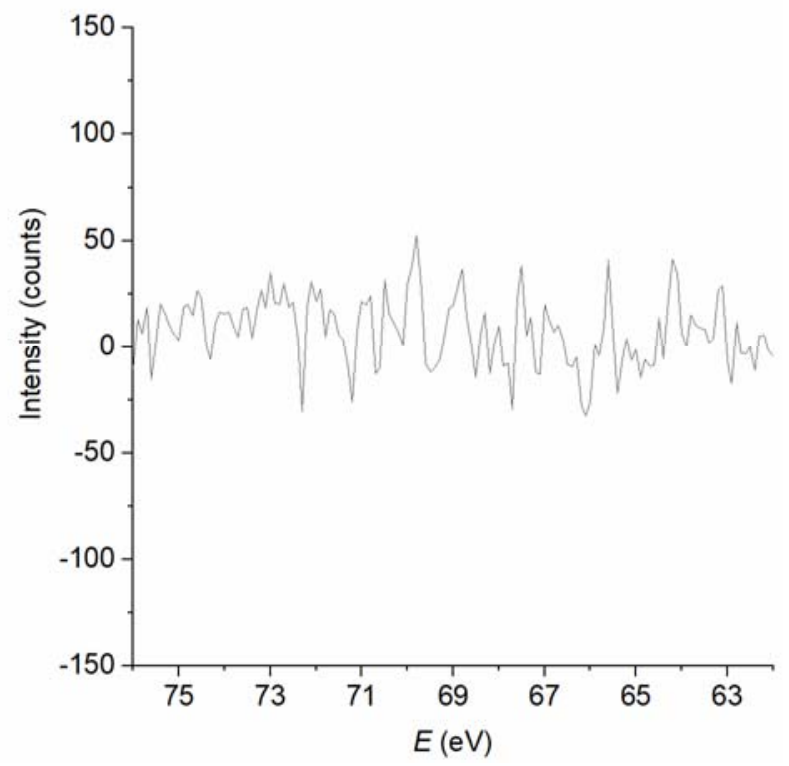

Figure S25. Br 3d XPS of (a) fresh mpg-CN. (b) recovered mpg-CN. 
a)

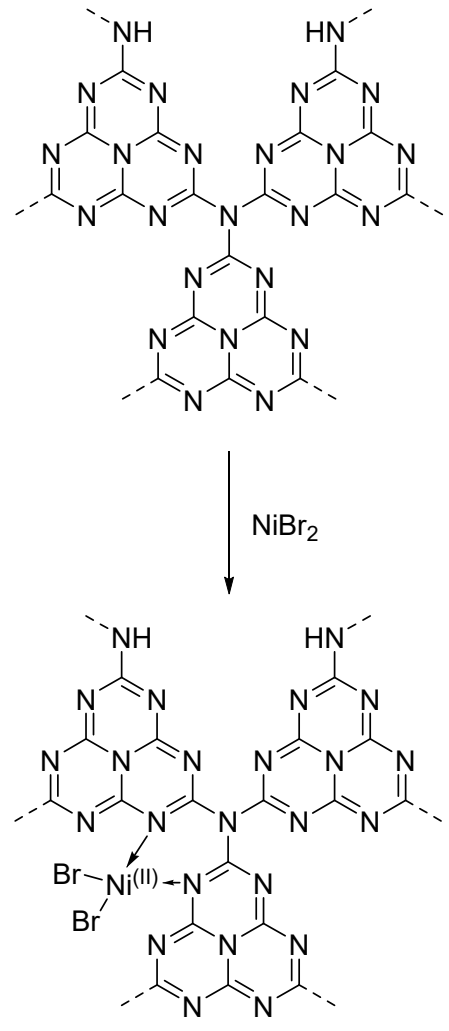

b)

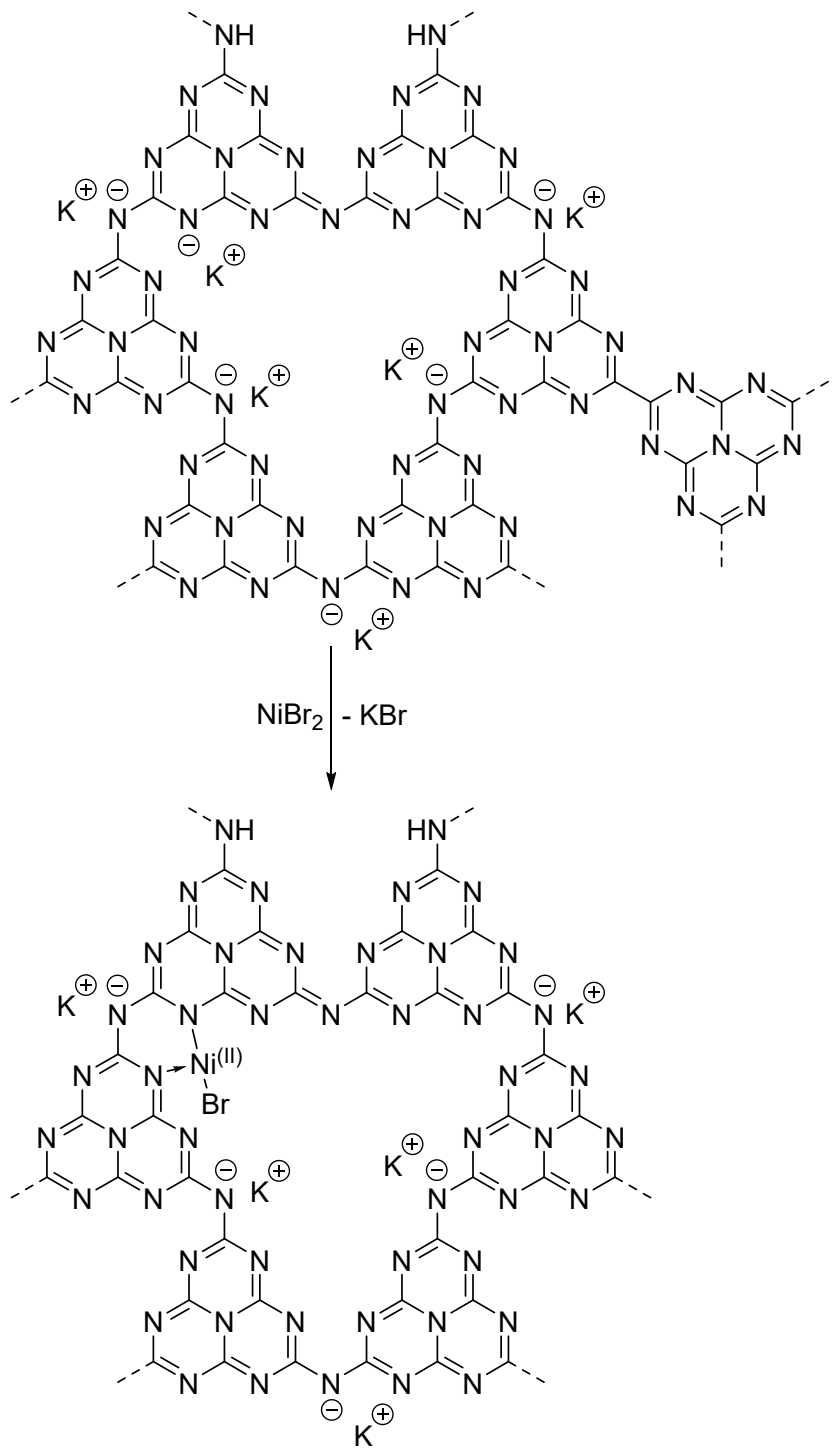

Figure S26. (a) Schematic representation of Ni salt coordination Covalent carbon nitride, such as mpg-CN. (b) ionic carbon nitride, such as $\mathrm{CN}-\mathrm{OA}-\mathrm{m}$. 


\section{Mechanistic Experiments}

$\left[(\right.$ bpy $) \mathrm{Ni}^{\mathrm{II}}(o$-tolyl $\left.) \mathrm{Br}\right](\mathbf{B})$ was synthesized following the literature procedure. ${ }^{20,21}$

Representative Procedure for experiments with catalytic[(bpy)NiII (o-tolyl)Br] complex II:

A $5 \mathrm{~mL}$ crimp vial equipped with a magnetic stirring bar was charged with, 1-bromo-2methylbenzene (34.2 mg, $0.2 \mathrm{mmol})$, mpg-CN (10.0 mg), [(bpy) Ni $\left.{ }^{\mathrm{II}}(\mathrm{o}-\mathrm{tolyl}) \mathrm{Br}\right](3.9 \mathrm{mg}, 5$ mol\%). Subsequently, $1.0 \mathrm{~mL}$ of $\mathrm{N}, \mathrm{N}$-dimethylacetamide (DMA) was added followed by addition of 2,6-lutidine ( $70 \mu \mathrm{L}, 0.6 \mathrm{mmol})$. The vial was sealed, and the reaction mixture was then introduced to a nitrogen atmosphere via "freeze-pump-thaw" cycles $(\times 3)$ with a syringe needle, keeping the vacuum approx. 5 mbar. It was irradiated with $5 \mathrm{~W} 455( \pm 15) \mathrm{nm}$ LEDs through the plane bottom side of the crimp vial and stirred intensely. The temperature was maintained at $30{ }^{\circ} \mathrm{C}$ by cooling with built-in cooling fan. After $48 \mathrm{~h}$, the crude product was analyzed by GC-FID using1,4-dimethoxybenzene as an external standard.

Table S10. Catalytic experiments with preformed [(bpy) $\mathrm{Ni}^{\mathrm{II}}(o-$ tolyl) $\mathrm{Br}]$ complex II.

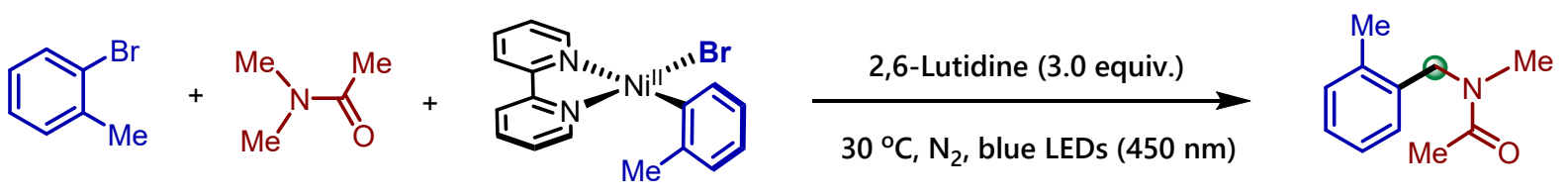

II

\begin{tabular}{ccc}
\hline Entry & $\begin{array}{c}\text { Deviation from the above } \\
\text { condition }\end{array}$ & Yield \\
\hline 1. & none & $5 \%$ \\
2. & With mpg-CN $\left(10 \mathrm{mg} \mathrm{mL}^{-1}\right)$ & $56 \%$ \\
\hline
\end{tabular}

$\underline{\text { Representative Procedure for experiments with stoichiometric [(bpy)NiI }(o-t o l y l) B r] \text { complex }}$ $\underline{\text { II: }}$

A $5 \mathrm{~mL}$ crimp vial equipped with a magnetic stirring bar was charged with, [(bpy) $\mathrm{Ni}^{\mathrm{II}}(o-$ tolyl)Br] (9.64 mg, $5 \mathrm{~mol} \%$ ). Subsequently, $1.0 \mathrm{~mL}$ of $N, N$-dimethylacetamide (DMA) was added followed by addition of 2,6-lutidine $(8.8 \mu \mathrm{L}, 0.075 \mathrm{mmol})$. The vial was sealed, and the reaction mixture was then introduced to a nitrogen atmosphere via "freeze-pump-thaw" cycles $(\times 3)$ with a syringe needle, keeping the vacuum approx. 5 mbar. It was irradiated with indicated light source through the plane bottom side of the crimp vial and stirred intensely. The 
temperature was maintained at $30^{\circ} \mathrm{C}$ by cooling with built-in cooling fan. After $48 \mathrm{~h}$, the crude product was analyzed by GC-FID using1,4-dimethoxybenzene as an external standard.

Table S11. Stoichiometric experiments with preformed [(bpy) $\mathrm{Ni}^{\mathrm{II}}(o$-tolyl)Br] complex II.<smiles>Cc1ccccc1N(Br)c1nc2ccccc2n1CBr</smiles>

II<smiles>CCCN(C)C(C)=O</smiles>
Photocatalyst

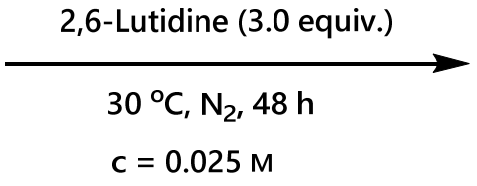<smiles>CC(=O)N(C)Cc1ccccc1C</smiles>

\begin{tabular}{cccc}
\hline Entry & Photocatalyst & Light source & Yield \\
\hline 1. & - & $340 \mathrm{~nm}$ & $17 \%$ \\
2. & - & $450 \mathrm{~nm}$ & $20 \%$ \\
3. & - & Dark & ND \\
$4 .^{a}$ & $\mathrm{Ru}(\mathrm{bpz})_{3} \mathrm{PF}_{6}\left(\mathrm{EM}^{*} / \mathrm{M}^{-}=+1.45 \mathrm{~V} \mathrm{Em} \lambda=591 \mathrm{~nm}\right)$ & $450 \mathrm{~nm}$ & $22 \%$ \\
$5 .^{a}$ & $(9-\mathrm{MesAcr}) \mathrm{ClO}_{4}\left(\mathrm{EM}^{*} / \mathrm{M}^{-}=+2.05 \mathrm{~V} \mathrm{Em} \lambda=590 \mathrm{~nm}\right)$ & $450 \mathrm{~nm}$ & $24 \%$ \\
\hline
\end{tabular}

${ }^{a} 10 \mathrm{~mol} \%$ photocatalyst was used.

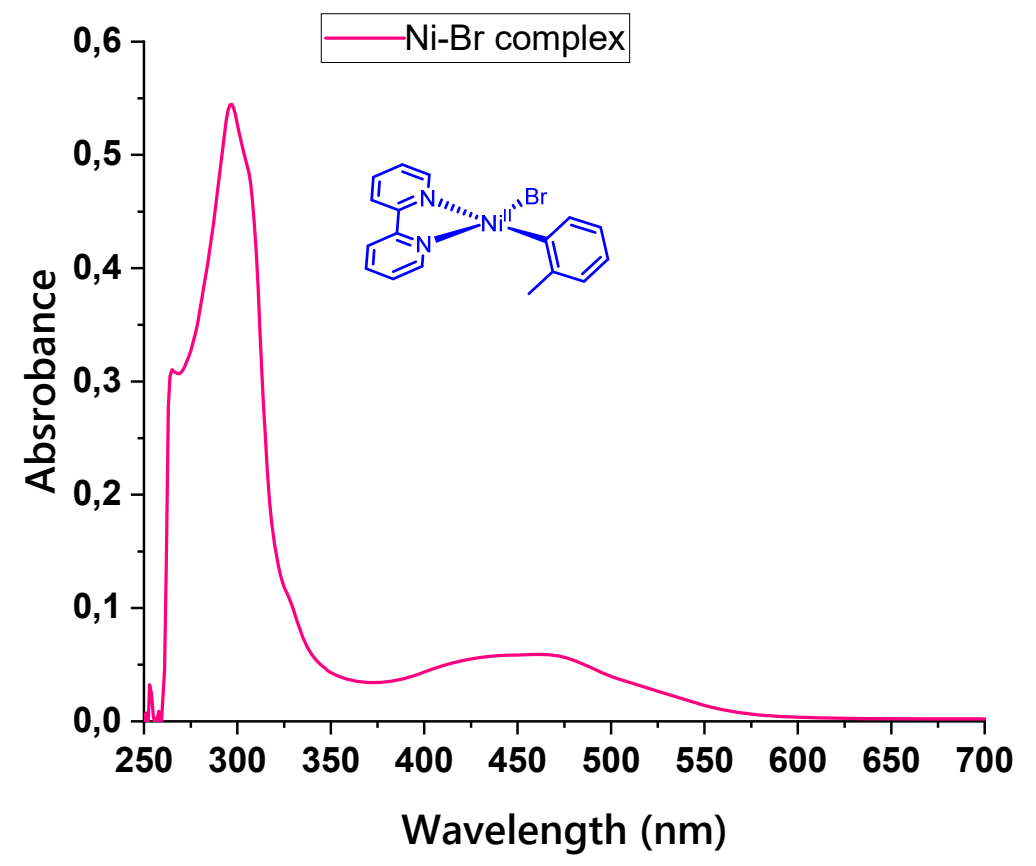

Figure S27. UV-Visible spectra of [(bpy) $\mathrm{Ni}^{\mathrm{II}}(o-$ tolyl $\left.) \mathrm{Br}\right]$ complex B in DMA. 


\section{Investigation of scalability in Continuous Flow reactors}

\subsection{Representative Procedure for Continuous Flow Investigations}

Flow reactions were conducted within a "Vapourtec reactor" (Figure S28, S29, S30), fitted with a pulsator unit (ProMinent (beta/4) pulsator unit with stainless steel 316 pump head) between a Vapourtec V3 slurry-handling pump and a $10 \mathrm{~mL}$ PTFE reactor coil housed within the Vapourtec UV-150 mirrored photochemical reactor cavity ${ }^{22}$. The blue LED manifold was positioned at the center of the coil resulting in a fully closed system. The flow path was first flushed clean with DMA degassed via Ar bubbling (30 min). A $50 \mathrm{~mL}$ crimp cap vial equipped with a stir bar was dried overnight and charged with 4-bromobenzonitrile (4.6 mmol, 1.0 equiv.), mpg-CN (10 mg mL $\left.{ }^{-1}\right), \mathrm{NiBr}_{2} \bullet$ glyme (5 mol\%) and 2,2'-bipyridine (5 mol\%) before placing under a nitrogen atmosphere. Afterwards, 2,6-lutidine (3.0 eq.) and DMA (23 mL) were added under nitrogen via a syringe. The reaction mixture was degassed via "freeze-pump-thaw" cycles $(\times 3)$, backfilled with $\mathrm{N}_{2}$ and stirred intensely under $\mathrm{N}_{2}$ protection. Then, blue LEDs (60 $\mathrm{W}$ input power, $24 \mathrm{~W}$ radiant power at $450 \mathrm{~nm})$ and the pulsator $(20 \%$ amplitude and $50 \%$ frequency) were switched on and the degassed reaction mixture was introduced via an inlet directly from the crimp cap vial through the slurry-handling V3 pump, through the pulsator and into the "Vapourtec reactor" with a $0.50 \mathrm{~mL} \mathrm{~min}{ }^{-1}$ flow rate. An adjustable BPR was set for a back pressure between 2-3 bar. First combined $20 \mathrm{~mL}$ of solvent (ca. $19 \mathrm{~mL}$ total reactor volume) and diluted reaction mixture was discarded. A $1 \mathrm{~mL}$ of sample was then collected for analysis of a 'single pass' reaction. After collecting the sample, the flow rate was stopped, and the outlet was connected directly to the reaction vial for recirculation. Argon was introduced via a syringe and the flow rate was returned. The argon protection needle was removed after a further 10 minutes. Temperature of the coil reactor was maintained at $c a .25{ }^{\circ} \mathrm{C}$ by a nitrogen flow through a canister filled with dry ice chips.

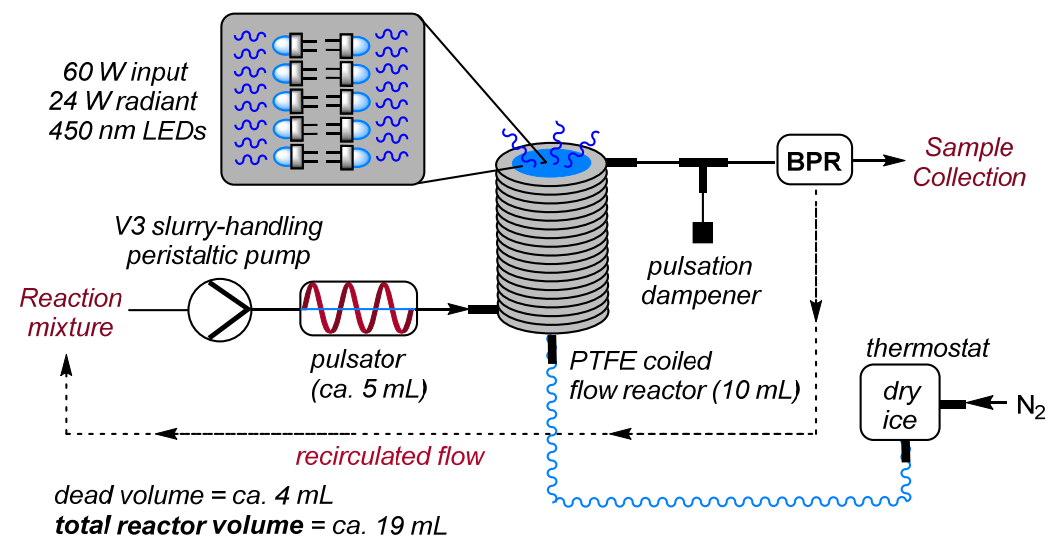

Figure S28. Schematic representation of "Vapourtec reactor" continuous flow setup. 


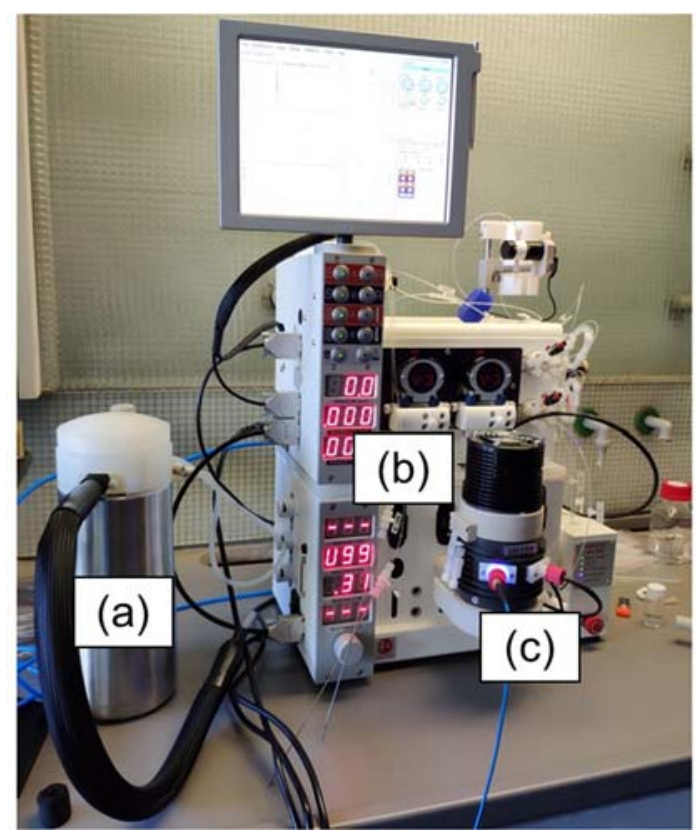

Figure S29. (a) Temperature control unit utilizing a flow of $\mathrm{N}_{2}$ over dry ice chips. (b) Vapourtec R-Series V3 peristaltic pumps. (c) Vapourtec UV-150 module consisting of a $10 \mathrm{~mL}$ PFA reaction coil and a Vapourtec LED module, $\lambda_{\max }=450 \mathrm{~nm}$, up to $60 \mathrm{~W}$ input power, $24 \mathrm{~W}$ radiant power at maximum current.

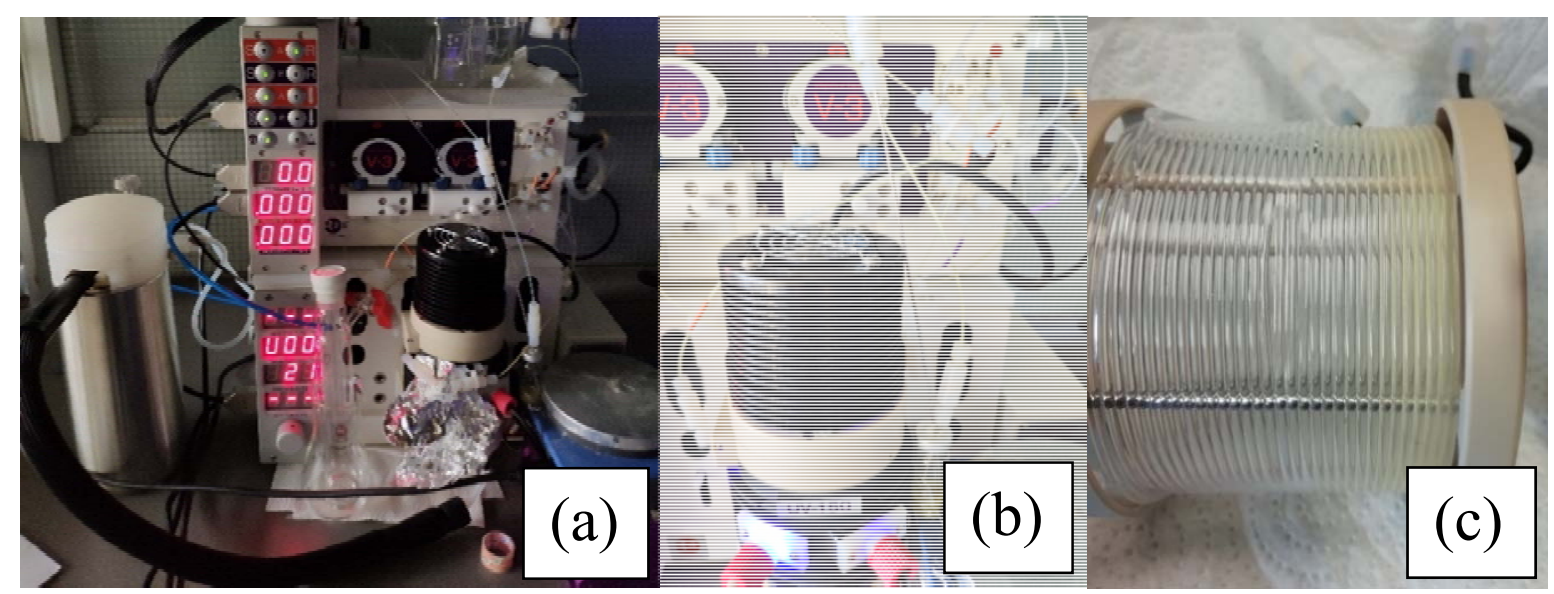

Figure S30. (a) Connection of the cooling unit to the UV-150 module prior to operation, temperature was set to $50{ }^{\circ} \mathrm{C}$ (external temperature). (b) Visually-even dispersion of mpg-CN photocatalyst across the laminar-type flow path (coiled tube reactor) before entry to the coil. (c) Sedimentation of mpg-CN within the laminar-type flow path $(10 \mathrm{~mL}$ PFA reactor coil). 


\subsection{Representative Procedure for Continuous Flow Investigations}

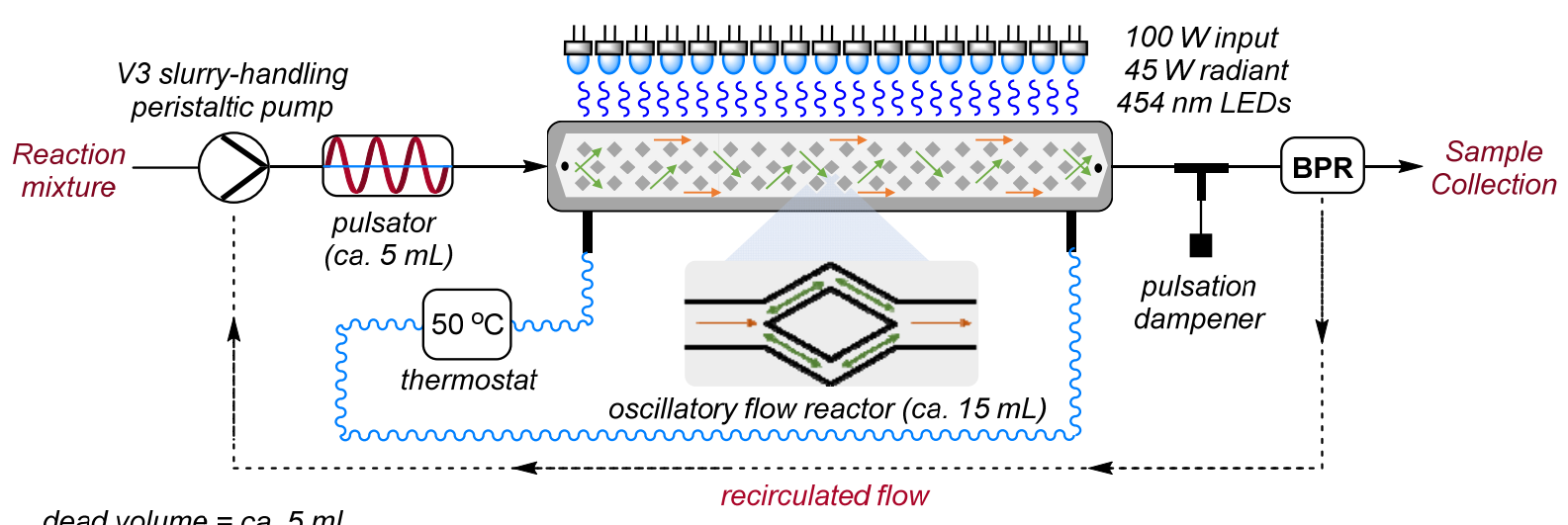

total reactor volume $=c a .25 \mathrm{~mL}$

Figure S31. Schematic representation of "HANU reactor" continuous flow setup.

Flow reactions were conducted within a "HANU reactor" (Figure S31, S32, S33), fitted with a pulsator unit (ProMinent (beta/4) pulsator unit with stainless steel 316 pump head) between a Vapourtec V3 slurry-handling pump and the $15 \mathrm{~mL}$ HANU reactor. The water-cooled blue LED manifold was positioned on top of the HANU reactor as a fully enclosed system. The flow path was first flushed clean with DMA degassed via Ar bubbling (30 min). A $50 \mathrm{~mL}$ crimp cap vial equipped with a stir bar was dried overnight and charged with 4-bromobenzonitrile (6.0 mmol, 1.0 equiv.), mpg-CN (10 $\left.\mathrm{mg} \mathrm{mL}^{-1}\right), \mathrm{NiBr}_{2} \bullet$ glyme ( $5 \mathrm{~mol} \%$ ) and 2,2'-bipyridine ( $5 \mathrm{~mol} \%$ ) before placing under a nitrogen atmosphere. Afterwards, 2,6-lutidine (3.0 eq.) and DMA (30 mL) were added under nitrogen via a syringe. The reaction mixture was degassed via "freeze-pump-thaw" cycles $(\times 3)$, backfilled with $\mathrm{N}_{2}$ and stirred intensely under $\mathrm{N}_{2}$ protection. Then, blue LEDs (100 $\mathrm{W}$ input power, $45 \mathrm{~W}$ radiant power at $454 \mathrm{~nm})$ and the pulsator $(20 \%$ amplitude and $50 \%$ frequency) were switched on and the degassed reaction mixture was introduced via an inlet directly from the crimp cap vial through the slurry-handling V3 pump, through the pulsator and into the "Vapourtec reactor" with a $0.75 \mathrm{~mL} \mathrm{~min}^{-1}$ flow rate. An adjustable BPR was set for a back pressure between 2-3 bar. First combined $25 \mathrm{~mL}$ of solvent and diluted reaction mixture (ca. $26 \mathrm{~mL}$ total reactor volume) was discarded. A $1 \mathrm{~mL}$ of sample was then collected for analysis of a 'single pass' reaction. After collecting the sample, the flow rate was stopped, and the outlet was connected directly to the reaction vial for recirculation. Argon was introduced via a syringe and the flow rate was returned. The argon protection needle was removed after a further 10 minutes. Temperature of the "HANU" reactor block was maintained at $c a .25{ }^{\circ} \mathrm{C}$ or ca. $50{ }^{\circ} \mathrm{C}$ by a thermostat recirculating heated distilled water. 
Alternatively, a customized PTFE-base oscillatory flow reactor ${ }^{23}$ (Figure S32) was employed to confirm if a material incompatibility issue of stainless steel 316 with the reaction mixture was responsible for poor conversion. Since the Teflon material is a strong thermal insulator, the heat generated by the LED manifold could not be removed and the flow path temperature could not be precisely controlled but was measured at ca. 33-43 ${ }^{\circ} \mathrm{C}$ (external temperature).

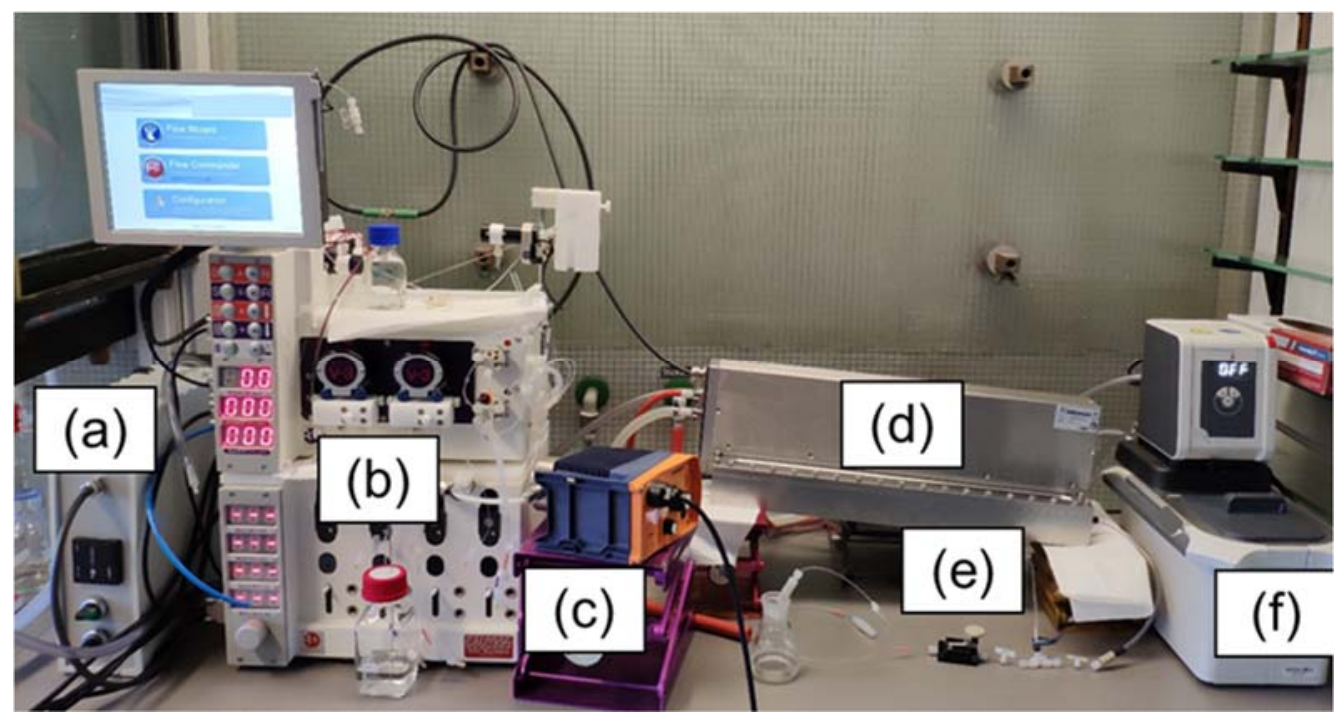

Figure S32. (a) Peschl Ultraviolet LED light intensity regulator. (b) Vapourtec R-Series V3 peristaltic pumps. (c) CreaFlow customized ProMinent (beta/4) pulsator unit with stainless steel 316 pump head (positioned as in Figure S27e during operation). (d) reactor module consisting of a water-cooled stainless steel 316 microstructured oscillatory baffled flow reactor (HANU ${ }^{\mathrm{TM}}$ reactor, $15 \mathrm{~mL}$ internal volume) and a water-cooled Peschl Ultraviolet LED module, $\lambda_{\max }=454$ $\mathrm{nm}$, up to $100 \mathrm{~W}$ input power, $45 \mathrm{~W}$ radiant power $\left(\mathrm{Fe}_{\mathrm{e}}\right)$ at maximum current. (e) Adjustable backpressure regulator (Vapourtec) following a pulsation dampener. (f) Thermostat for temperature control of the $\mathrm{HANU}^{\mathrm{TM}}$ flow path set at 25 or $50^{\circ} \mathrm{C}$ (external temperature).

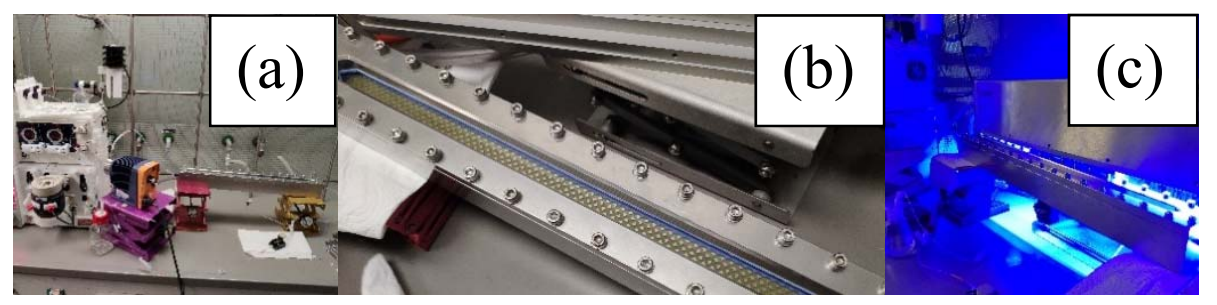

Figure S33. (a) Vertical positioning of pulsator unit. (b) Visually-even dispersion of mpg-CN photocatalyst across the turbulent-type flow path before irradiation. (c) Representative picture of the operating Peschl LED module (during operation, the LED module was placed directly atop the $\mathrm{HANU}^{\mathrm{TM}}$ reactor). 


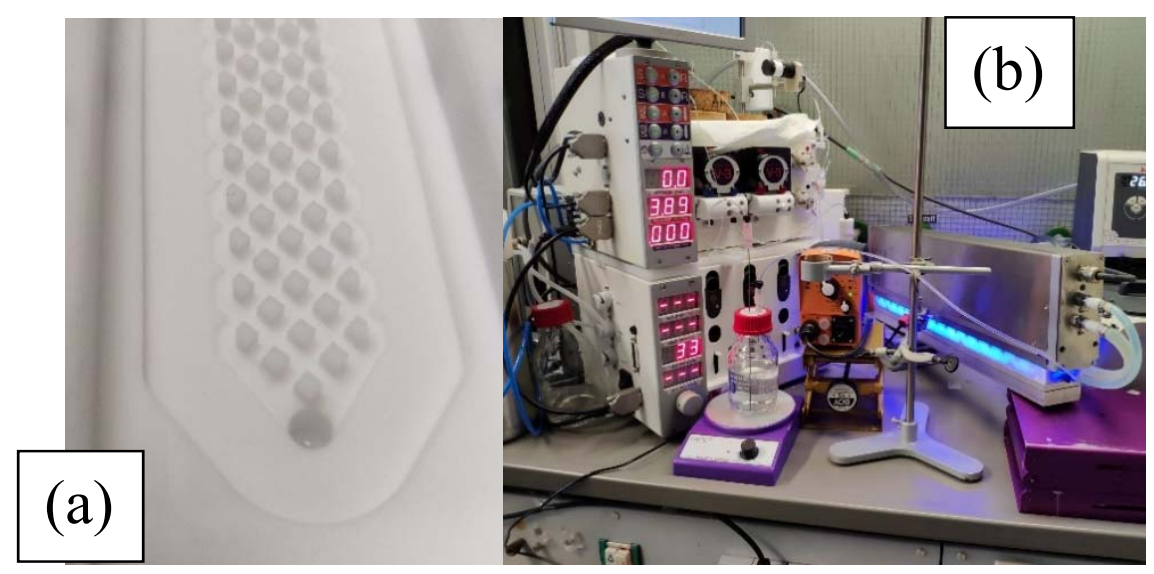

Figure S34. (a) PTFE-modified flow oscillatory flow path; (b) Assembled system as per Figure S12g-h including a ProMinent (beta 4-BT4b) pulsator unit with a PTFE pump head. External temperature of the flow path varied from $33-43^{\circ} \mathrm{C}$.

The model reaction of 4-bromobenzonitrile (A) with DMA was chosen for scalability evaluation in continuous flow reactors. We first examined the use of a commercially-available microstructured oscillatory flow reactor $\left(\mathrm{HANU}^{\mathrm{TM}}\right.$ reactor, CreaFlow), in combination with a pulsator unit, whose synergy is a state-of-the-art technology for processing heterogeneous photocatalytic reactions in a continuous flowing suspension/slurry. ${ }^{23}$ The microstructured cubic static mixing elements are especially designed, in tandem with pulsation, to ensure homogeneity in the suspension of carbon nitride particles and to prevent settling and flow channel fouling at any given flow rate. Initial conditions mirrored a previous report employing a different carbon nitride catalyst (“CN-OA-m”, reported particle size distribution $0.45-875 \mathrm{~mm}$ ) which afforded the optimal residence time distribution and yield for a photocatalytic Buchwald-Hartwig-type amination. ${ }^{24}$ Namely, $0.2 \mathrm{M}$ concentration of $\mathbf{A}, 3.33 \mathrm{mg} \mathrm{mL}^{-1} \mathrm{mpg}-\mathrm{CN}$ loading, $5 \mathrm{~mol} \%$ loading of both nickel catalyst and ligand, temperature of $50{ }^{\circ} \mathrm{C}$, pulsation amplitude of $20 \%$ and frequency of 50\% and residence time of 20 minutes. LED manifold input and radiant power employed ( $\lambda_{\max }=454 \mathrm{~nm}, 45 \mathrm{~W}$ radiant power at maximum current) were very similar to this report ( $\lambda_{\max }=460 \mathrm{~nm}, 34 \mathrm{~W}$ radiant power at maximum current were reported). Given the proximity of the LEDs to the flow channel occupying a thin film (HANU channel depth $=c a$. $2.0 \mathrm{~mm}$ ) of $15 \mathrm{~mL}$ and assuming an ideal system in which no optical power is lost from the reactor, one can estimate a 'radiant power density' of $3.0 \mathrm{~W} \mathrm{~mL}^{-1}$. However, we were surprised to find absolutely no conversion of $\mathbf{A}$ under these conditions after a single pass through the reactor (Table S12, entry 1). Recirculating for $16 \mathrm{~h}$ afforded ca. $6 \%$ conversion (entry 2 ). 
Table S12. Investigation of scalability in Continuous Flow reactors.

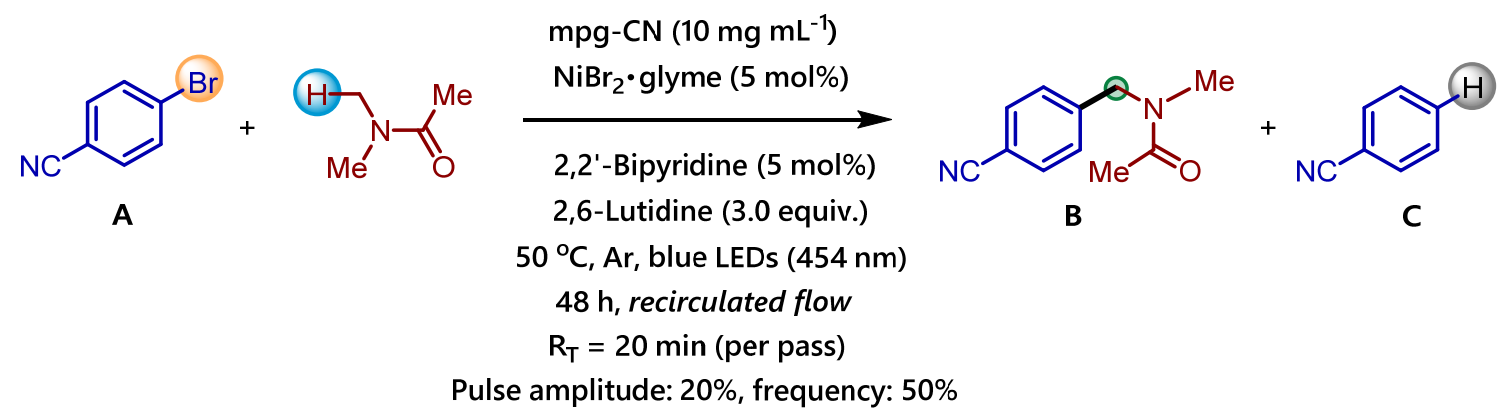

\begin{tabular}{|c|c|c|c|}
\hline Entry $^{a}$ & Reactor System & Deviation from Conditions & Ratio A:B: $\mathbf{C}^{b}$ \\
\hline 1. & HANU $^{\mathrm{TM}}$ SS 316 OFR & $\begin{array}{c}3.33 \mathrm{mg} / \mathrm{mL} \text { mpg-CN, } \\
\text { 'single pass', } \mathrm{R}_{\mathrm{T}}=20 \mathrm{~min}\end{array}$ & n.r. \\
\hline 2. & HANU $^{\mathrm{TM}} \mathrm{SS} 316$ OFR & $3.33 \mathrm{mg} / \mathrm{mL} \mathrm{mpg-CN}, 16 \mathrm{~h}$ & $94: 5: 1$ \\
\hline 3. & HANU $^{\mathrm{TM}} \mathrm{SS} 316$ OFR & 'single pass', $\mathrm{R}_{\mathrm{T}}=20 \mathrm{~min}$ & n.r. \\
\hline 4. & HANU $^{\mathrm{TM}} \mathrm{SS} 316$ OFR & $15 \mathrm{~h}$ & $88: 10: 2$ \\
\hline 5. & HANU $^{\mathrm{TM}} \mathrm{SS} 316$ OFR & $48 \mathrm{~h}$ & $65: 27: 8$ \\
\hline 6. & HANU $^{\mathrm{TM}} \mathrm{SS} 316$ OFR & $110 \mathrm{~h}$ & $39: 45: 16$ \\
\hline 7. & HANU $^{\mathrm{TM}} \mathrm{SS} 316$ OFR & $\begin{array}{l}\text { 3-acetoxyquinuclidine ( } 1.5 \text { eq.) } \\
\text { instead of } 2,6 \text {-lutidine }\end{array}$ & $74: 17: 9$ \\
\hline 8. & Teflon OFR & (different reactor material) & 79:9:12 \\
\hline 9. & HANU $^{\mathrm{TM}} \mathrm{SS} 316$ OFR & 'pulsated batch' & $66: 30: 4$ \\
\hline 10. & HANU $^{\mathrm{TM}} \mathrm{SS} 316$ OFR & 'pulsated batch', $25^{\circ} \mathrm{C}$ & $77: 14: 9$ \\
\hline 11. & HANU $^{\mathrm{TM}} \mathrm{SS} 316$ OFR & $30 \%$ light intensity & $89: 7: 4$ \\
\hline $12^{c}$. & Vapourtec UV-150 & $-(39 h)$ & $58: 28: 14$ \\
\hline $13^{c}$. & Vapourtec UV-150 & $-(110 \mathrm{~h})$ & $42: 34: 24$ \\
\hline
\end{tabular}

${ }^{a}$ Reaction Condition: 4-bromobenzonitrile 'A' (6 mmol), mpg-CN (10 mg mL $\left.{ }^{-1}\right), \mathrm{NiBr}_{2} \cdot{ }^{\cdot}$ glyme (5 mol\%), 2,2'bipyridine (5 mol\%), 2,6-lutidine (3.0 equiv.), DMA ( $0.2 \mathrm{M}$ with respect to $\mathbf{A}) .{ }^{b}$ Reaction component ratios were determined by GC-FID and are expressed as a relative ratio of their total peak areas (i.e. do not account for other products). ${ }^{c} 4.6 \mathrm{mmol}$ of ' $\mathbf{A}$ ' was used in DMA ( $0.2 \mathrm{M}$ with respect to $\left.\mathbf{A}\right)$. n.r., no reaction.

Increasing mpg-CN loading to $10 \mathrm{mg} \mathrm{mL}^{-1}$ still gave no conversion after a single pass through the reactor (entry 3 ), while recirculation for $15 \mathrm{~h}$ gave $c a .12 \%$ conversion, double that of the $3.33 \mathrm{mg} \mathrm{mL}^{-1}$ loading (entry 4). Further recirculation of this reaction for $48 \mathrm{~h}$ and $110 \mathrm{~h}$ (almost 5 days!) increased conversion to $c a .35 \%$ and ca. 61\%, respectively (entries 5-6). Suspecting that the herein-proposed bromine radicals as HAT agents may be interacting with the stainless steel (SS 316) material of the reactor, we substituted 2,6-Lutidine base with 3acetoxyquinuclidine whose radical cation is a HAT agent known to engage $\alpha$-amido $\mathrm{C}-\mathrm{H}$ bonds. ${ }^{25}$ However, conversion did not improve (ca. $25 \%$ after $48 \mathrm{~h}$, entry 7 ). We also performed the reaction in a modified oscillatory flow reactor path made from Teflon, and employed a pulsator with a PTFE pump head, removing all contact of the reaction mixture with SS 316 . However, conversion did not improve (ca. $21 \%$ after 48 h, entry 8 ). In light of these results, a 
material compatibility issue with SS 316 can be ruled out. Next, we operated the HANU reactor in a 'batch-type' mode, filling the $15 \mathrm{~mL}$ reactor with reaction mixture, ceasing the flow and pulsating within the illuminated reactor. After $48 \mathrm{~h}$ (entry 9), the result was almost identical to result of recirculated flow with a $0.75 \mathrm{~mL} \mathrm{~min}^{-1}$ flow rate, indicating that a flowing stream of reaction mixture offers no benefit to conversion. Decreasing light intensity to $30 \%$ of the maximum value (ca. $13.5 \mathrm{~W}$ radiant power) gave $30 \%$ of the combined relative amounts of products as was provided by the $100 \%$ intensity (entry 10 vs entry 5 ). Conversion may therefore be photon-limited, but on the assumption of the aforementioned direct proportionality between intensity and conversion, one would require ca. $135 \mathrm{~W}$ of radiant power across the flow path for full conversion and $48 \mathrm{~h}$ of reaction time at $50{ }^{\circ} \mathrm{C}$. Since $50{ }^{\circ} \mathrm{C}$ is a sub-optimal temperature for the reaction described herein (as it provides increased amounts of reduced product), the corresponding reaction at $\mathrm{rt}$ would require an even longer reaction time. We then attempted to process the reaction mixture within another commercial photochemical flow reactor; a Vapourtec UV-150 photochemical reactor equipped with a $10 \mathrm{~mL}$ PTFE coil placed within a mirrored cavity and surrounding an LED manifold $\left(\lambda_{\max }=450 \mathrm{~nm}, 60 \mathrm{~W}\right.$ input power. Interestingly, under the same conditions $\left(10 \mathrm{mg} / \mathrm{mL}^{-1} \mathrm{mpg}-\mathrm{CN}, 5 \mathrm{~mol} \%\right.$ nickel catalyst and ligand, $50{ }^{\circ} \mathrm{C}$ ) we observed $42 \%$ conversion after $39 \mathrm{~h}$ of recirculating (no products were detected upon a single pass through the reactor), which was at a first glance superior to the result after $48 \mathrm{~h}$ of recirculating in the HANU reactor (entry 12 vs entry 5). However, over extended periods of operation we observed accumulation of mpg-CN due to sedimentation in the PTFE coil, leading to a blockage and loss of detected pressure after $40 \mathrm{~h}$. When the reaction was restarted, the rate of conversion was noticeably lower (see Figure S35, final graph and entry 12) ultimately resulting in a similar conversion after $110 \mathrm{~h}$ as the HANU reactor with its superior mixing properties (see Figure S35, first graph and entry 5). Due to the different mixing properties of the reactors, it is inappropriate to make comparisons of the kinetic profiles shown in Figure S35 at this stage. Given the proximity of the LEDs to the PTFE coil reactor which provides a thin film (o.d. $=1.6 \mathrm{~mm}$, i.d. $=1.0 \mathrm{~mm}$ ) of $10 \mathrm{~mL}$ and assuming an ideal system in which no optical power is lost from the reactor, one can estimate a 'radiant power density' of $2.4 \mathrm{~W} \mathrm{~mL}^{-1}$. This 'radiant power density' is $0.8 \mathrm{x}$ that of the $3.0 \mathrm{~W} \mathrm{~mL}^{-1}$ estimated from the HANU reactor. Given that the Vapourtec UV-150 total reactor volume requires $0.8 \mathrm{x}$ that of the HANU reactor and reactions are compared at identical concentration, we are confident in the comparability of results between Vapourtec UV-150 reactor and HANU reactor systems in this study. 

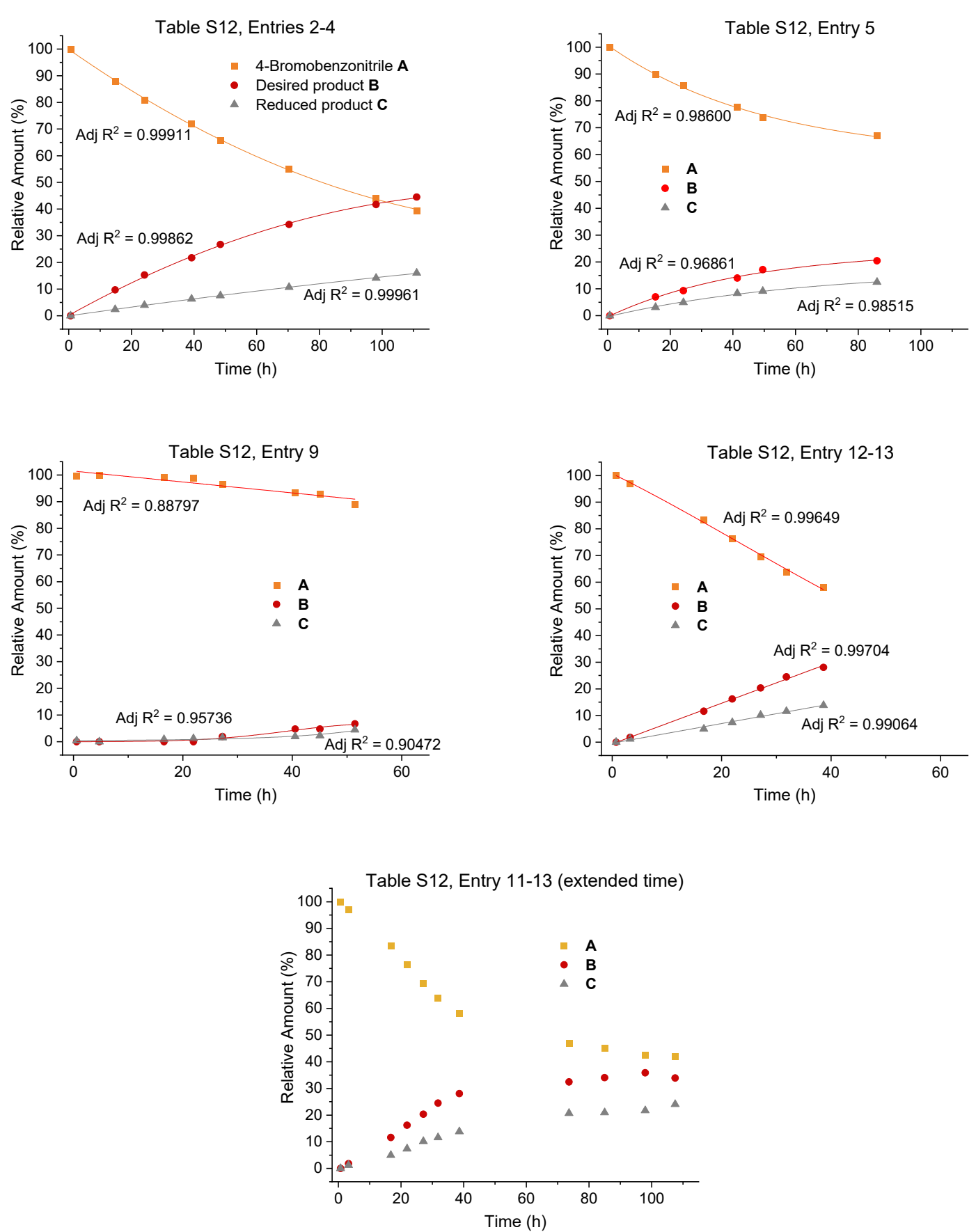

Figure S35. Time-course profiles for Continuous Flow reaction investigations in Table S12.

We investigated increasing the nickel and bipyridine catalyst loadings to elucidate their impact in batch mode, in search of more suitable reaction conditions for continuous flow processing (Table S13). We note that (unless otherwise stated) reactions performed in Table S13 used OSRAM Oslon SSL 80 LDCQ7P-2U3U LT1960 LEDs ( $\lambda=440 \mathrm{~nm}( \pm 15 \mathrm{~nm}), 1.5 \mathrm{~W}$ LEDs (optical power $500 \mathrm{~mW}$ ), which are weaker in optical power than the LEDs used generally in the study but nonetheless afforded full conversion after $105 \mathrm{~h}$ at $\mathrm{rt}$ and after $85 \mathrm{~h}$ at $50{ }^{\circ} \mathrm{C}$ under the reaction conditions described in General Procedures 8.1 and 8.2 (0.2 M A in DMA, $10 \mathrm{mg}$ 
$\mathrm{mL}^{-1} \mathrm{mpg}-\mathrm{CN}, 5 \mathrm{~mol} \%$ nickel and bipyridine catalyst, 3.0 eq. 2,6-Lutidine). Different reaction conditions are compared with these LEDs in Table S13.

Table S13. Investigation of reaction kinetics in batch mode.

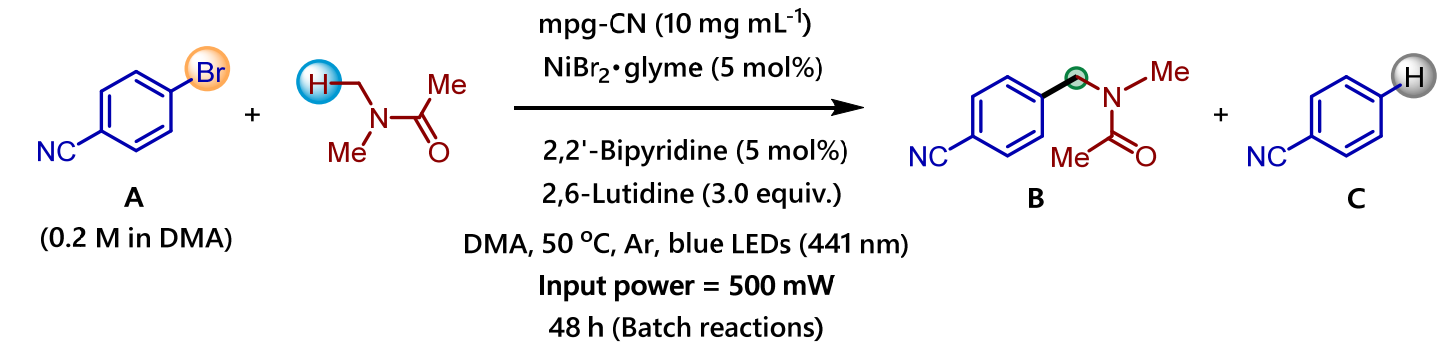

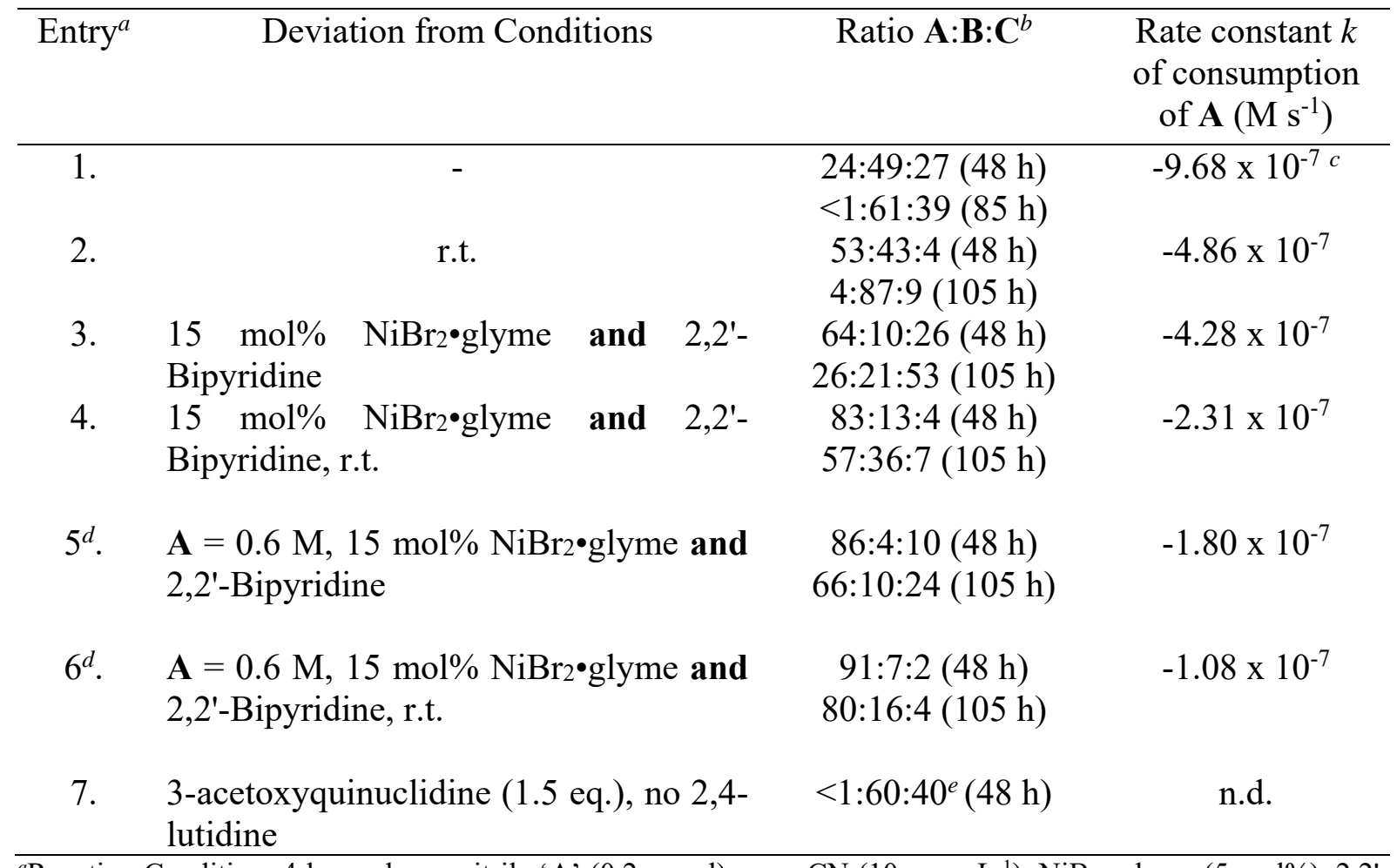

${ }^{a}$ Reaction Condition: 4-bromobenzonitrile 'A' (0.2 mmol), mpg-CN (10 mg mL $\left.{ }^{-1}\right), \mathrm{NiBr}_{2} \cdot \mathrm{glyme}(5 \mathrm{~mol} \%), 2,2^{\prime}-$ bipyridine ( $5 \mathrm{~mol} \%$ ), 2,6-lutidine (3.0 equiv.), DMA ( $0.2 \mathrm{M}$ with respect to A). ${ }^{b}$ Reaction component ratios were determined by GC-FID and are expressed as a relative ratio of their total peak areas (i.e. do not account for other

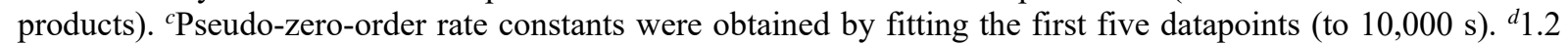
mmol of ' $\mathbf{A}$ ' was used in DMA ( $0.2 \mathrm{M}$ with respect to $\mathbf{A}) .{ }^{e}$ Reaction performed using OSRAM OSLON $®$ SSL 80 GD CS8PM1.14 LEDs ( $\lambda=451 \mathrm{~nm}( \pm 15 \mathrm{~nm}), 5 \mathrm{~W}$ optical power). n.d. not determined

Clearly, the reaction is operating under a photon-limited regime, given the comparison of different LED intensities (Table S12, entries 5 vs 11 and the fact that reactions took $>80 \mathrm{~h}$ to complete using $500 \mathrm{~mW}$ optical power LEDs compared to the 'standard' $5 \mathrm{~W}$ optical power LEDs used for the substrate scope and optimization studies). Reaction rates also show a temperature dependence; reactions proceed faster at $50{ }^{\circ} \mathrm{C}$ but lead to more debromination product (Table S13, entry 1 vs 2). We were intrigued to observe an apparent change in reaction kinetic profile from zero-order behaviour to pseudo-zero-order behaviour in the consumption 

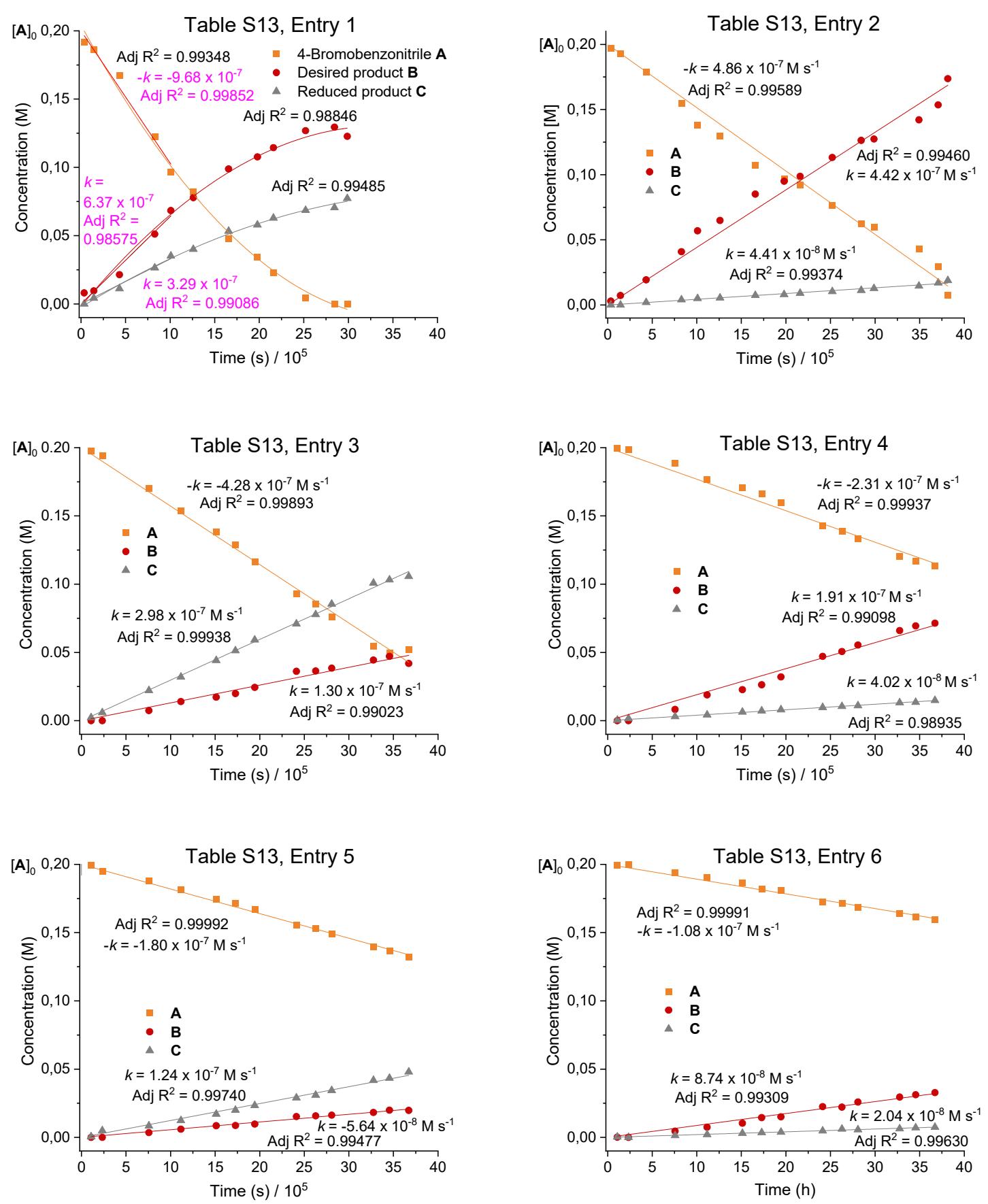

Figure S36. Time-course profiles for batch reaction investigations in Table S13.

of $\mathbf{A}$ and formation of $\mathbf{B}$ and $\mathbf{C}$ from $25^{\circ} \mathrm{C}$ to $50{ }^{\circ} \mathrm{C}$ (see Figure S36, first and second graph, entry 2 and entry 1 of Table S13). Given these that temperature is the only different factor, the zero-order dependence observed at $25{ }^{\circ} \mathrm{C}$ is not related to the abundance of light (both reactions are likely photon-limited in regime). Zero-order kinetic behaviour is typical of heterogeneous catalytic reactions ${ }^{26}$ in which active sites are saturated by absorbed reactant molecules and has been reported elsewhere for mpg- $\mathrm{CN}$ in both non-photocatalytic and dual-photocatalytic chemistry. ${ }^{27,28}$ As the reaction reaches full conversion, active sites become more available on 
the mpg-CN surface resulting in a final curvature of the kinetic behaviour. Zero-order behaviour in heterogeneous catalysis is well-known to result from the existence of an absorption equilibrium (equation 1) which is followed by a single, slow surface reaction step that is ratedetermining (equation 2$)^{29}$

$$
\begin{aligned}
& {[\mathrm{Ni}]_{\text {liquid }} \stackrel{\leftarrow}{\rightarrow}[\mathrm{Ni}]_{\text {adsorbed }} \quad ; \text { equilibrium }} \\
& {[\mathrm{Ni}]_{\text {adsorbed }} \rightarrow \text { products }}
\end{aligned}
$$

In this case, the rate law can be defined for consumption of 4-bromobenzonitrile $\mathbf{A}$ as equation 3. Integration from $t=0\left([\mathbf{A}]_{0}\right)$ to time $t=t\left([\mathbf{A}]_{t}\right)$ as per equation 4 leads to equation 5 which is a straight-line plot whose gradient $=-k$. The rate constant was thereby calculated for all plots in Table S13.

$$
\begin{gathered}
-\frac{d[\boldsymbol{A}]}{d t}=-k[\boldsymbol{A}] \\
-\int_{t=0}^{t=t} d[\boldsymbol{A}]=-k \int_{t=0}^{t=t} d t \\
{[\boldsymbol{A}]_{t}=[\boldsymbol{A}]_{0}-k t}
\end{gathered}
$$

At higher temperatures, we tentatively ascribe the shift in zero-order kinetic behaviour to pseudo-zero-order kinetic behaviour as one of three possibilities: (1) the activation energy of the surface-based rate-determining slow process (presumably involving adsorbed [Ni] complexes) is overcome, promoting desorption of 'products' from the mpg-CN surface and freeing up active sites of the mpg-CN catalyst; (2) a shift in the equilibrium of adsorption at the heterogeneous surface allowing more [Ni] complex whose reaction is rate-determining to engage with the active site; (3) An off-cycle direct reduction of $\mathbf{A}$ by a photoactive species is increased, providing a radical anion that traps the nickel as reported by Pieber, Seeberger et al. ${ }^{28}$ Proposal (3) is consistent with: i) the increase in abundance of dehydrohalogenated product $\mathbf{C}$ at $50{ }^{\circ} \mathrm{C}$, ii) the fractional rate dependence of aryl halide on the heterogeneous rate law (entries 5,6 vs entries 3,4) discussed below and iii) the redox potential of $\mathbf{A}\left(E^{\mathrm{p}_{\text {red }}}=-1.75 \mathrm{~V}\right.$ vs. $\mathrm{SCE}$ in $\left.\mathrm{DMF} / 0.1 \mathrm{M}^{\mathrm{n}} \mathrm{Bu}_{4} \mathrm{~N} \cdot \mathrm{BF}_{4}\right)^{30}$ being within almost within reach of photoexcited mpg-CN (up to $-1.5 \mathrm{~V}$ vs. SCE), albeit a slightly endergonic SET process that may be accessible at 50 ${ }^{\circ} \mathrm{C}^{31}$. Interestingly, increasing the $[\mathrm{Ni}]$ catalyst (+ ligand) loading by $3 \mathrm{x}$ (at the same concentration of $\mathbf{A}$ ) increased the relative amount of debromination product and halved the reaction rate (Table S13, entries 3,4 vs entries 1,2). This contrasts to Pieber and Seeberger's previous study involving decarboxylative arylation of $\mathrm{N}$-Boc-protected pyrrole with 4iodomethylbenzoate, which found that increasing [Ni] catalyst (+ ligand) increased the rate of 
reaction in a photon-unlimited regime of mpg-CN photocatalysis or had no impact on the rate of reaction when in a photon-limited regime. ${ }^{28}$ In moving from $5 \mathrm{~mol} \%$ to $15 \mathrm{~mol} \%$ of the [Ni] catalyst, the pseudo-zero-order behaviour also shifted to represent zero-order behaviour. One could rationalize this with a (Le Chatelier's principle) shift in the equilibrium to remove liquidphase [Ni] complexes in preference of adsorbed [Ni] complexes, saturating the mpg-CN active sites. If the [Ni] complex whose reaction is rate-determining has to compete with other offcycle or non-rate-determining on-cycle [Ni] complexes for mpg-CN active sites, the overall rate would slow. Increasing concentration of 4-bromobenzonitrile $\mathbf{A}$ to $0.6 \mathrm{M}$ further slowed reaction conversion (entries 5,6 vs entries 3,4). The fractional dependence on haloarene concentration is consistent with that reported by Pieber, Seeberger et al. ${ }^{28}$

Whether the reaction mechanism proceeds via Pathway A or Pathway B (see main manuscript and Section 7 for experiments supporting Pathway B), both mechanisms contain two steps where an [Ni] complex engages with the mpg-CN surface either in SET or EnT. In particular, we note that EnT processes are reported to be extremely efficient at very short distances ${ }^{32}$ and so effective adsorption to the mpg-CN surface may be crucial. While further investigations are underway in our laboratories, we tentatively propose that continuous flow results in (a) faster moving liquid phase and (b) a slower-moving mpg-CN solid particles. The asynchrous/divorced flow rates of (a) and (b) are unsupportive of adsorption of [Ni] complexes onto the mpg-CN surface. Pulsated flow and efficient mixing may also be disruptive to the adsorption processes on this particular morphology (mpg-CN) of carbon nitride photocatalyst. In batch mode, [Ni] complexes and mpg-CN are confined in space, resulting in a more uniform and constant flux of [Ni] complexes to mpg-CN.

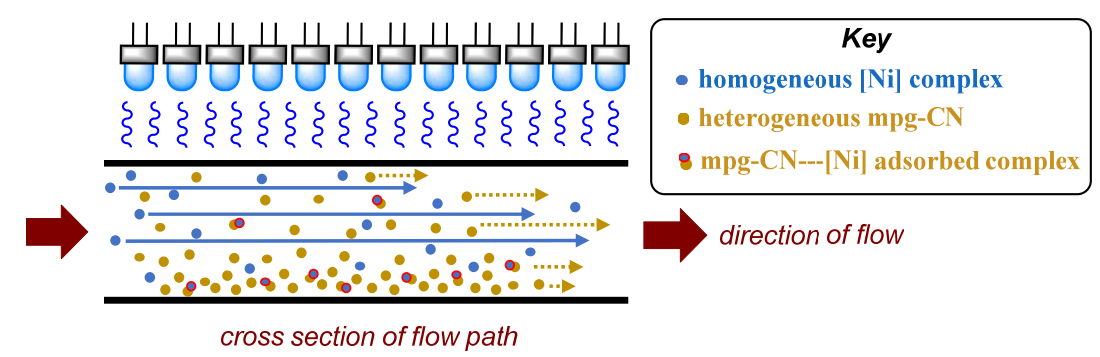

Figure S37. Pictorial representation of the divorced time-domains resulting from different flow rates of homogeneous [Ni] complexes and heterogeneous mpg-CN.

In conclusion, this study presents a rare example of how continuous flow is a not a viable solution for scale-up of a heterogeneous photocatalytic reaction. Two different commerciallyavailable photochemical flow reactors especially designed to handle and irradiate suspensions were compared (Table S14) and neither outcompeted the actual gram $\mathrm{h}^{-1}$ productivity of a batch 
reaction in the "Tauchschacht Photoreactor" on a ca. 6 mmol scale $(83 \%$ of $\mathbf{B}$ after $60 \mathrm{~h}$, a concentration of $0.1 \mathrm{M}$ was required by the Tauchschacht reactor compared to standard smallscale batch experiments and flow experiments in to maintain efficient reactor cooling). Even if one were to assume the relative amount (\%) of product (Figure S35) tracks with the yield, the maximum productivities at $0.2 \mathrm{M}$ of $\mathbf{A}$ in both flow systems would be ca. $6.3 \mathrm{mg} \mathrm{h}^{-1} \mathbf{B}$ (Table

Table S14. Actual productivity, yield and LED input power comparison of batch and flow systems.

\begin{tabular}{|c|c|c|c|}
\hline Reactor & Tauchshacht $^{a}$ & Vapourtec UV-150 & HANU SS 316 \\
\hline Processing mode & Batch & $\begin{array}{c}\text { Pulsated Flow, } \\
\text { laminar-type }\end{array}$ & $\begin{array}{c}\text { Pulsated Flow, } \\
\text { oscillatory baffled }\end{array}$ \\
\hline Conversion (\%) & & $35^{e}$ & $42^{f}$ \\
\hline Yield (\%) & $100 \%$ & $28^{e}$ & $27^{f}$ \\
\hline Productivity of B $\left(\mathrm{mg} \mathrm{h}^{-1}\right)$ & 15.6 & 6.2 & 6.3 \\
\hline Productivity of B $\left(\mathrm{mg} \mathrm{day}^{-1}\right)$ & 374.5 & 148.8 & 151.2 \\
\hline Input LED power & $12 \mathrm{~W}$ & $60 \mathrm{~W}$ & $100 \mathrm{~W}$ \\
\hline
\end{tabular}

${ }^{a}$ Based on isolated yield after 60 h. ${ }^{b}$ Assuming yield tracks with relative amount $(\% \mathbf{B})$ detected by the calibrated GC FID method as an estimation. ${ }^{c}$ Reactions conducted at $50^{\circ} \mathrm{C}$, reactions at rt provided poorer conversion. ${ }^{d}$ Based on the relative amount of starting material left $\left(100\right.$ - \%A) determined by the calibrated GC FID method. ${ }^{e} 39 \mathrm{~h}$. ${ }^{f 48}$ h.

S14). Since 'single-pass' conditions do not afford any conversion, further optimization is unlikely to remedy this to a level where it could be synthetically useful. Recirculated flow is fundamentally limited in scale-up and would be too time-consuming to be synthetically useful. Finally, while energy efficiency is not usually critically discussed in reports scaling-up photochemical reactions in continuous flow, we highlight the difference in LED input power required by the three systems (not taking into consideration the additional demand for resources, such as electrical, water, and $\mathrm{N}_{2}$ in cooling the hi-power LEDs). We note that while the benefits of continuous flow are usually obvious in scale-up of photochemical processes, this is not always the case ${ }^{33}$ and batch chemistry can be a viable scale-up option for certain chemistries. 


\section{Supporting references}

1. Ghosh, I. et al. Organic semiconductor photocatalyst can bifunctionalize arenes and heteroarenes. Science 365, 360 (2019).

2. Thurston, J. H., Hunter, N. M. \& Cornell, K. A. Preparation and characterization of photoactive antimicrobial graphitic carbon nitride $\left(\mathrm{g}-\mathrm{C}_{3} \mathrm{~N}_{4}\right)$ films. RSC Advances 6, 42240-42248 (2016).

3. Gao, D., Liu, Y., Liu, P., Si, M. \& Xue, D. Atomically Thin B doped $\mathrm{g}_{-} \mathrm{C}_{3} \mathrm{~N}_{4}$ Nanosheets: High-Temperature Ferromagnetism and calculated Half-Metallicity. Sci. Rep. 6, 35768 (2016).

4. Wang, Y. et al. Linker-controlled polymeric photocatalyst for highly efficient hydrogen evolution from water. Energy Environ. Sci. 10, 1643-1651 (2017).

5. Chang, F. et al. Enhanced visible-light-driven photocatalytic performance of porous graphitic carbon nitride. Appl. Surf. Sci. 358, 270-277 (2015).

6. Dontsova, D. et al. Triazoles: A New Class of Precursors for the Synthesis of Negatively Charged Carbon Nitride Derivatives. Chem. Mater. 27, 5170-5179 (2015).

7. Dai, H. et al. Synthesis and characterization of graphitic carbon nitride submicrospheres using microwave method under mild condition. Diam. Relat. Mater. 38, 109-117 (2013).

8. Zhu, B., Xia, P., Li, Y., Ho, W. \& Yu, J. Fabrication and photocatalytic activity enhanced mechanism of direct Z-scheme g- $\mathrm{C}_{3} \mathrm{~N}_{4} / \mathrm{Ag}_{2} \mathrm{WO}_{4}$ photocatalyst. Appl. Surf. Sci. 391, 175-183 (2017).

9. Zhang, H. et al. Flower-like Cobalt Hydroxide/Oxide on Graphitic Carbon Nitride for Visible-Light-Driven Water Oxidation. ACS Appl. Mater. Interfaces 8, 35203-35212 (2016).

10. Cao, S., Low, J., Yu, J. \& Jaroniec, M. Polymeric Photocatalysts Based on Graphitic Carbon Nitride. Adv. Mater. 27, 2150-2176 (2015).

11. Wang, X. et al. A metal-free polymeric photocatalyst for hydrogen production from water under visible light. Nat. Mater. 8, 76-80 (2009).

12. Jorge, A. B. et al. $\mathrm{H}_{2}$ and $\mathrm{O}_{2}$ Evolution from Water Half-Splitting Reactions by Graphitic Carbon Nitride Materials. J. Phys. Chem. C 117, 7178-7185 (2013).

13. Savateev, A. et al. Potassium Poly(Heptazine Imide): Transition Metal-Free Solid-State Triplet Sensitizer in Cascade Energy Transfer and [3+2]-cycloadditions. Angew. Chem. Int. Ed. 59, 15061-15068 (2020).

14. Shen, Y., Gu, Y. \& Martin, R. $\mathrm{sp}^{3} \mathrm{C}-\mathrm{H}$ Arylation and Alkylation Enabled by the Synergy of Triplet Excited Ketones and Nickel Catalysts. J. Am. Chem. Soc. 140, 12200-12209 (2018).

15. Shields, B. J. \& Doyle, A. G. Direct $\mathrm{C}\left(\mathrm{sp}^{3}\right)-\mathrm{H}$ Cross Coupling Enabled by Catalytic Generation of Chlorine Radicals. J. Am. Chem. Soc. 138, 12719-12722 (2016).

16. Pieber, B. et al. Semi-heterogeneous Dual Nickel/Photocatalysis using Carbon Nitrides: Esterification of Carboxylic Acids with Aryl Halides. Angew. Chem. Int. Ed. 58, 95759580 (2019).

17. Gisbertz, S., Reischauer, S. \& Pieber, B. Overcoming limitations in dual photoredox/nickel-catalysed $\mathrm{C}-\mathrm{N}$ cross-couplings due to catalyst deactivation. Nat. Catal. 3, 611-620 (2020).

18. Zhang, G. et al. Optimizing Optical Absorption, Exciton Dissociation, and Charge Transfer of a Polymeric Carbon Nitride with Ultrahigh Solar Hydrogen Production Activity. Angew. Chem. Int. Ed. 56, 13445-13449 (2017).

19. Savateev, A. \& Antonietti, M. Ionic Carbon Nitrides in Solar Hydrogen Production and Organic Synthesis: Exciting Chemistry and Economic Advantages. ChemCatChem 11, 6166-6176 (2019). 
20. Ting, S. I. et al. 3d-d Excited States of Ni(II) Complexes Relevant to Photoredox Catalysis: Spectroscopic Identification and Mechanistic Implications. J. Am. Chem. Soc. 142, 5800-5810 (2020).

21. Standley, E. A., Smith, S. J., Müller, P. \& Jamison, T. F. A Broadly Applicable Strategy for Entry into Homogeneous Nickel(0) Catalysts from Air-Stable Nickel(II) Complexes. Organometallics 33, 2012-2018 (2014).

22. https://www.vapourtec.com/products/flow-reactors/.

23. http://www.creaflow.be/products.

24. Rosso, C. et al. An oscillatory plug flow photoreactor facilitates semi-heterogeneous dual nickel/carbon nitride photocatalytic C-N couplings. React. Chem. Eng. 5, 597-604 (2020).

25. Shaw, M. H., Shurtleff, V. W., Terrett, J. A., Cuthbertson, J. D. \& MacMillan, D. W. C. Native functionality in triple catalytic cross-coupling: $\mathrm{sp}^{3} \mathrm{C}-\mathrm{H}$ bonds as latent nucleophiles. Science 352, 1304-1308 (2016).

26. Gaya, U. Heterogeneous photocatalysis using inorganic semiconductor solids. (SPRINGER, 2016).

27. Ayán-Varela, M. et al. Investigating the Dispersion Behavior in Solvents, Biocompatibility, and Use as Support for Highly Efficient Metal Catalysts of Exfoliated Graphitic Carbon Nitride. ACS Appl. Mater. Interfaces 7, 24032-24045 (2015).

28. Malik, J. A., Madani, A., Pieber, B. \& Seeberger, P. H. Evidence for Photocatalyst Involvement in Oxidative Additions of Nickel-Catalyzed Carboxylate $O$-Arylations. $J$. Am. Chem. Soc. 142, 11042-11049 (2020).

29. Murzin, D. Y. On Langmuir kinetics and zero order reactions. Catal. Commun. 9, 18151816 (2008).

30. Reche, I., Mena, S., Gallardo, I. \& Guirado, G. Electrocarboxylation of halobenzonitriles: An environmentally friendly synthesis of phthalate derivatives. Electrochim. Acta 320, 134576 (2019).

31. Vogt, D. B., Seath, C. P., Wang, H. \& Jui, N. T. Selective C-F Functionalization of Unactivated Trifluoromethylarenes. J. Am. Chem. Soc. 141, 13203-13211 (2019).

32. Welin, E. R., Le, C., Arias-Rotondo, D. M., McCusker, J. K. \& MacMillan, D. W. C. Photosensitized, energy transfer-mediated organometallic catalysis through electronically excited nickel(II). Science 355, 380-385 (2017).

33. Elliott, L. D. et al. Batch versus Flow Photochemistry: A Revealing Comparison of Yield and Productivity. Chem. Eur. J. 20, 15226-15232 (2014). 


\section{${ }^{1} \mathrm{H}$ and ${ }^{13} \mathrm{C}$ Spectra}
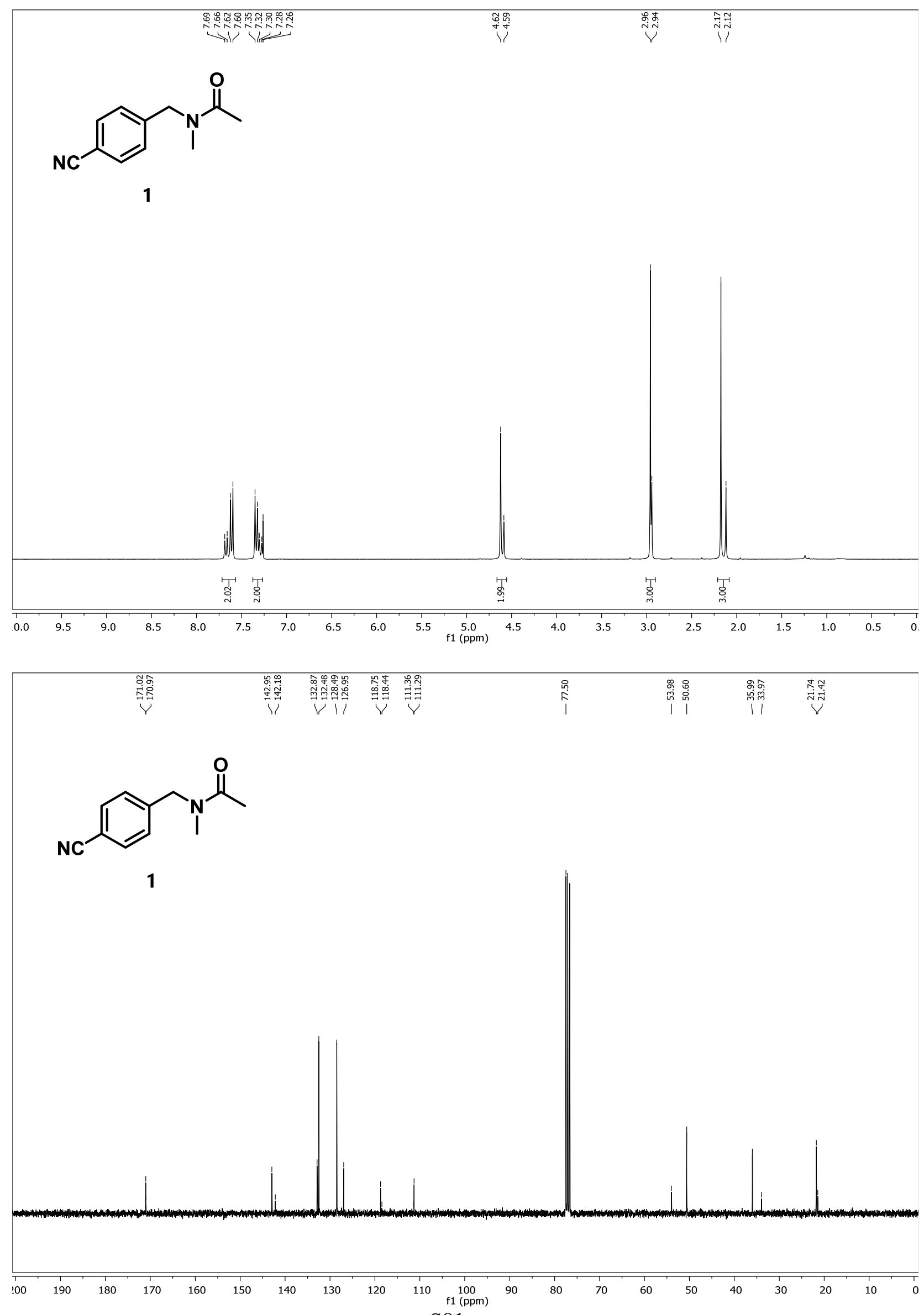

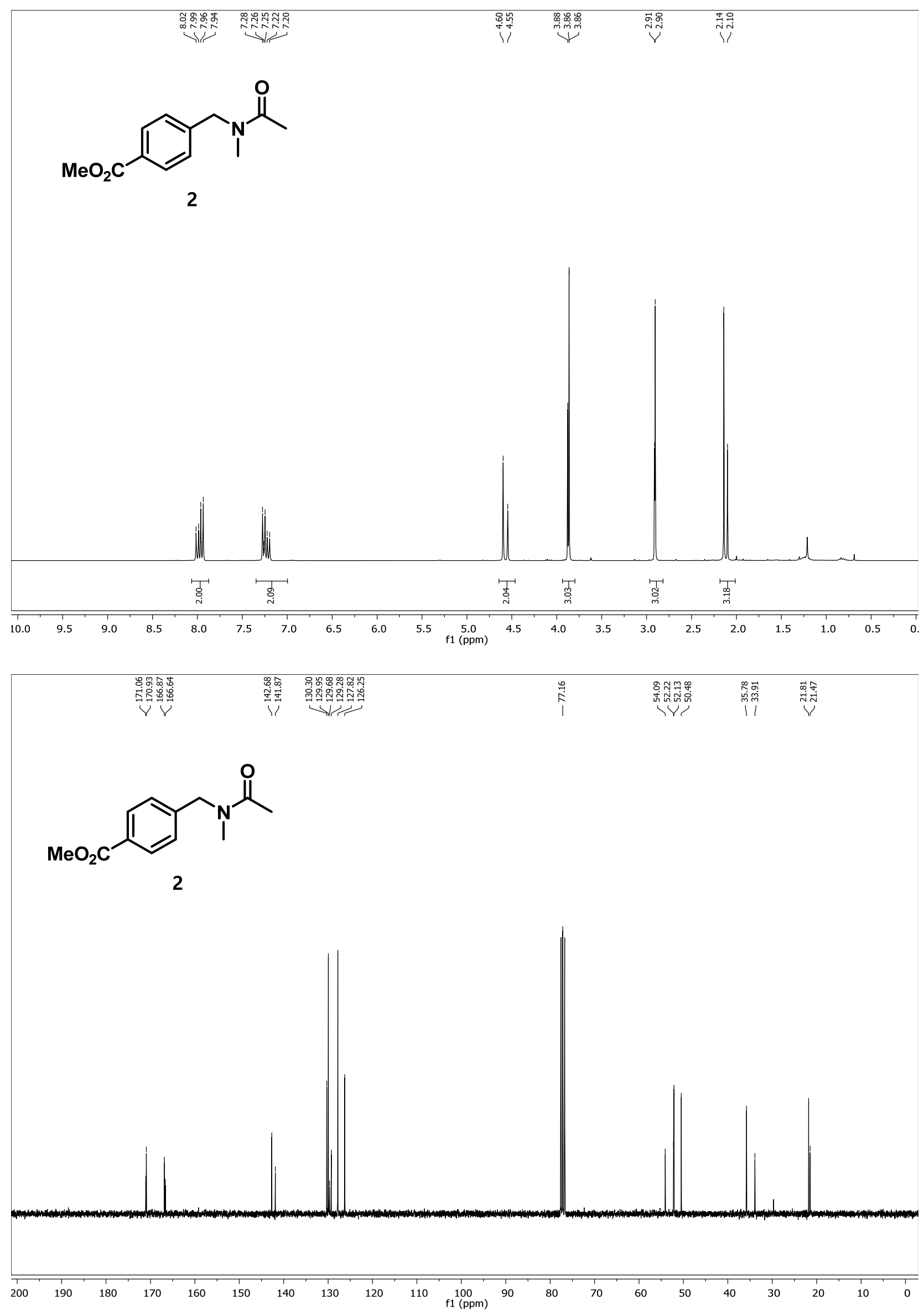

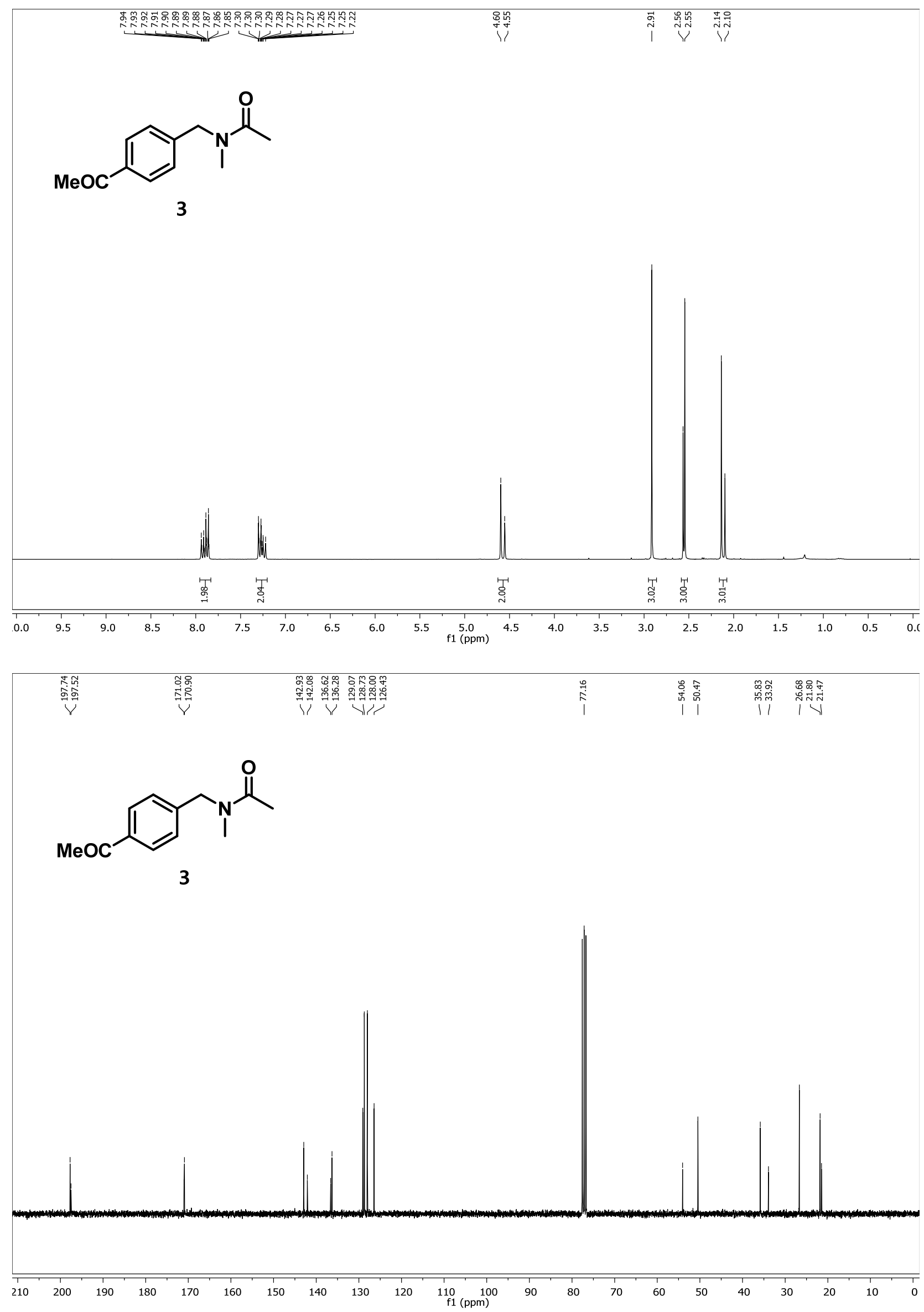

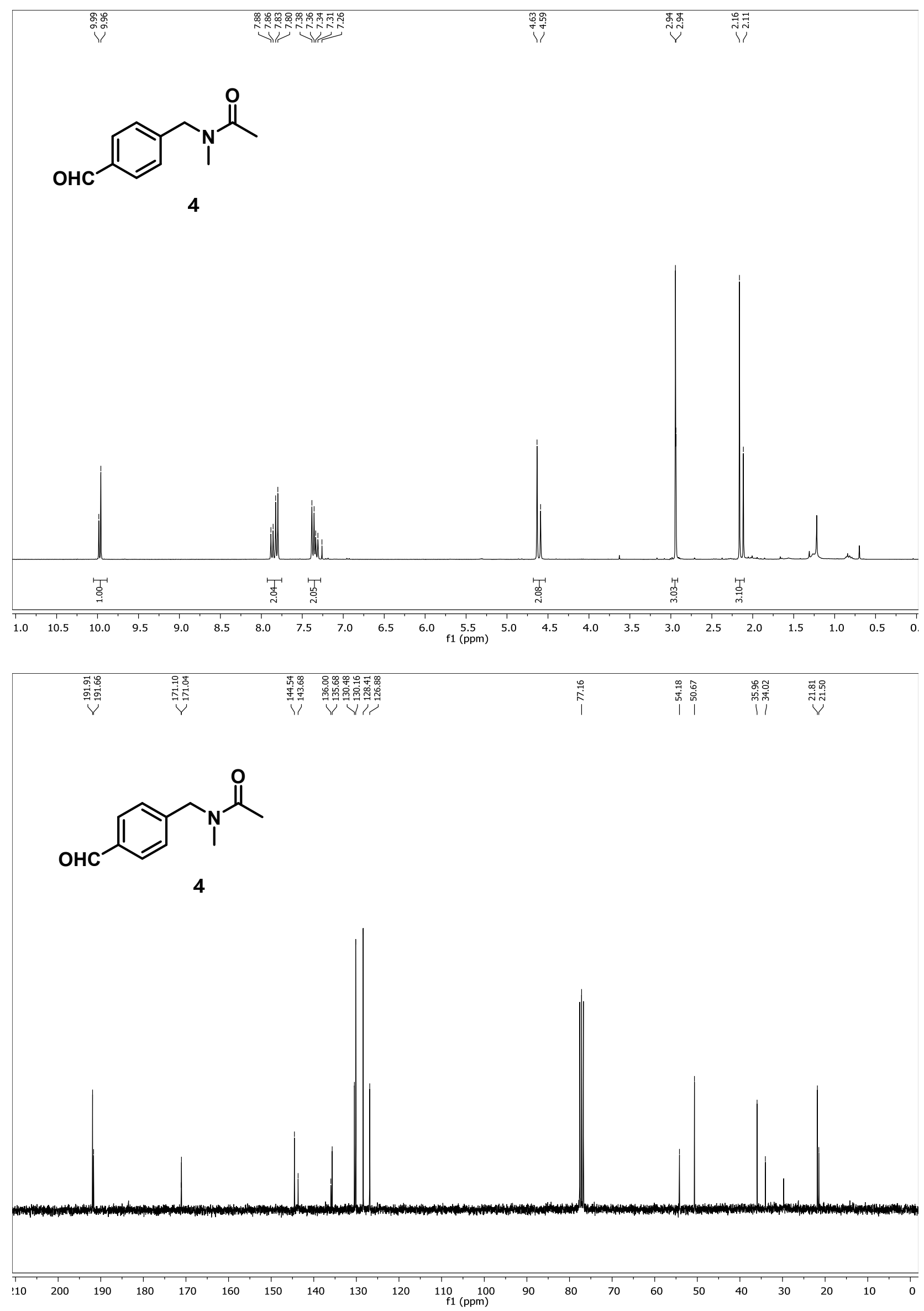

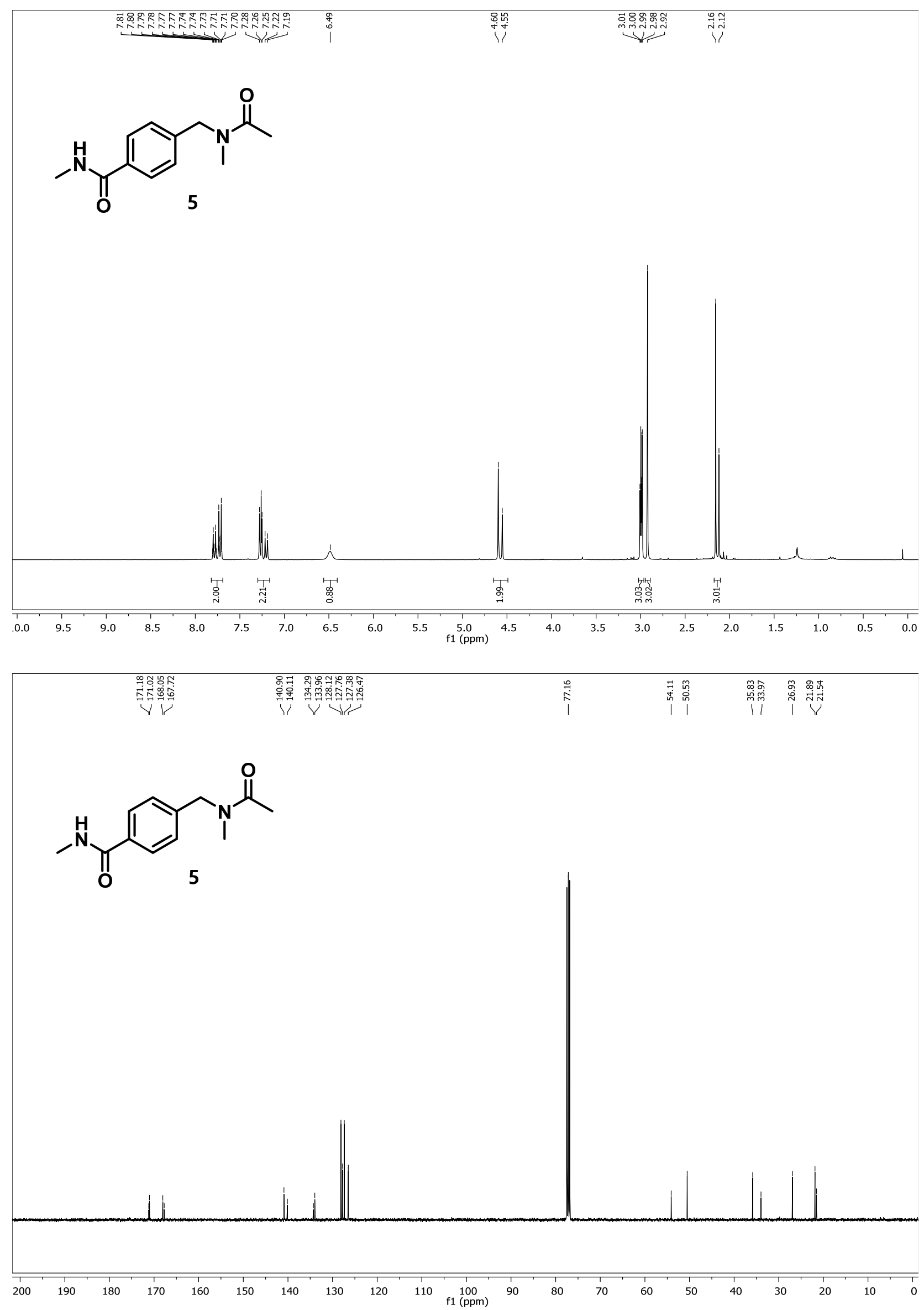

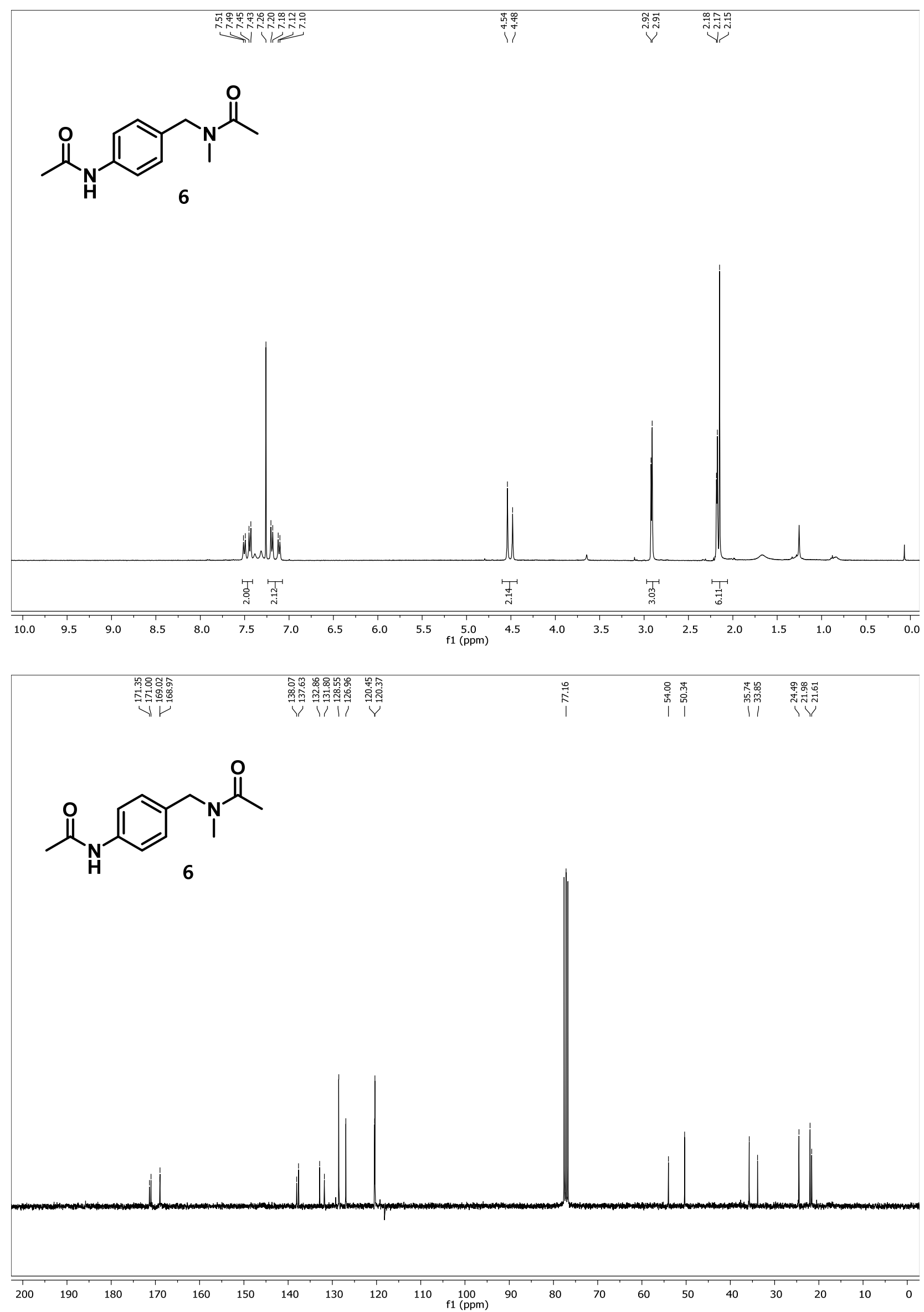

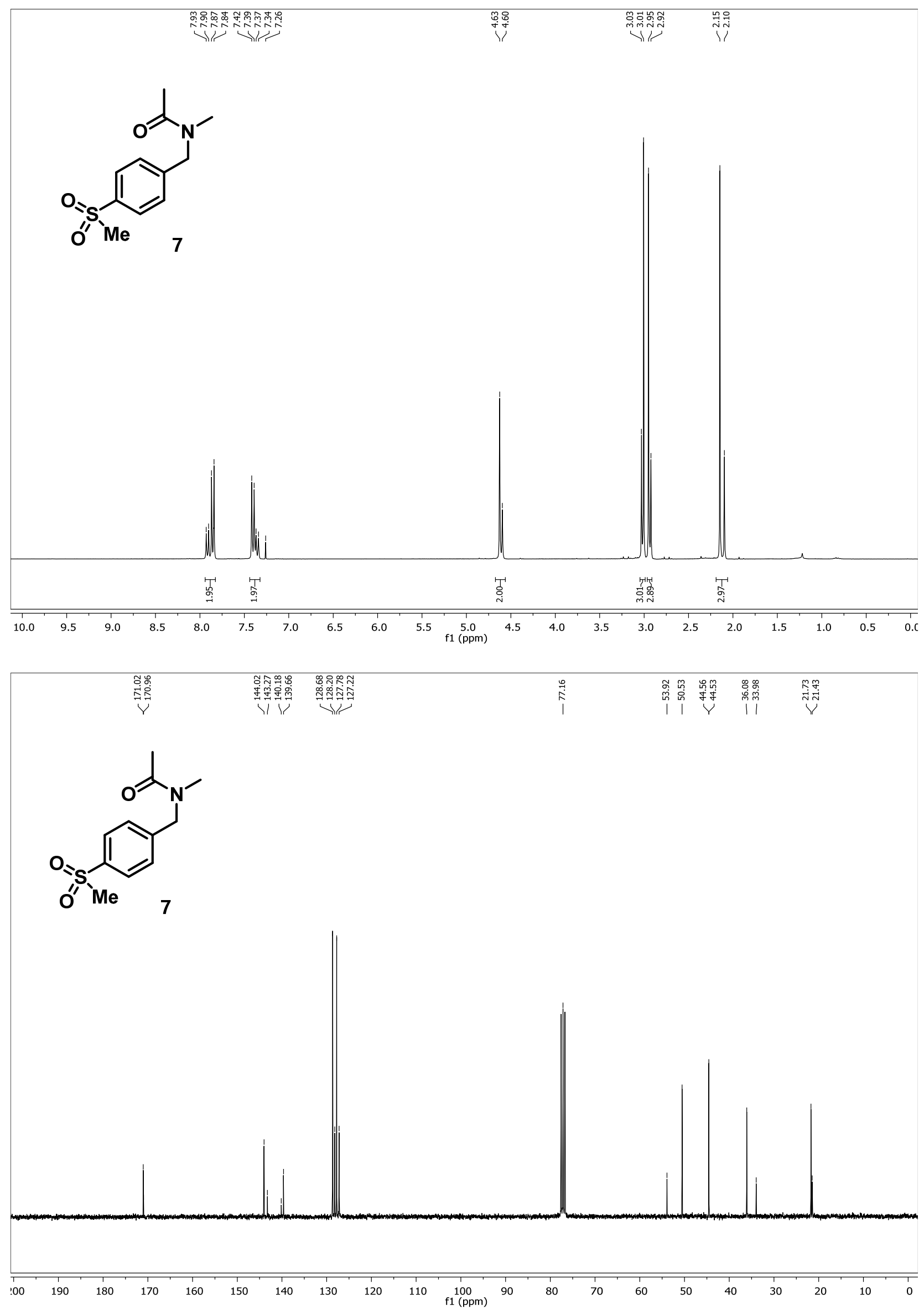

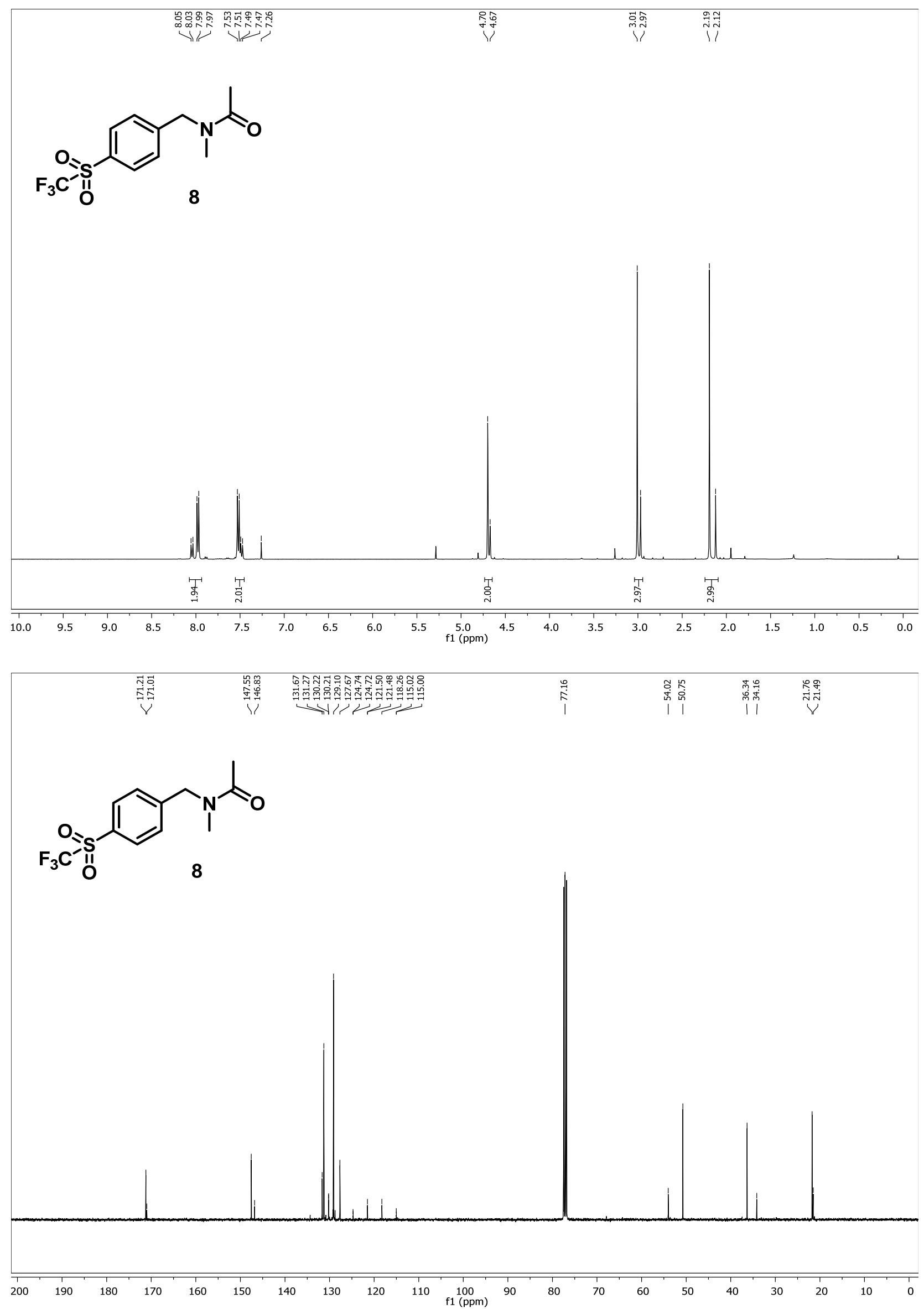

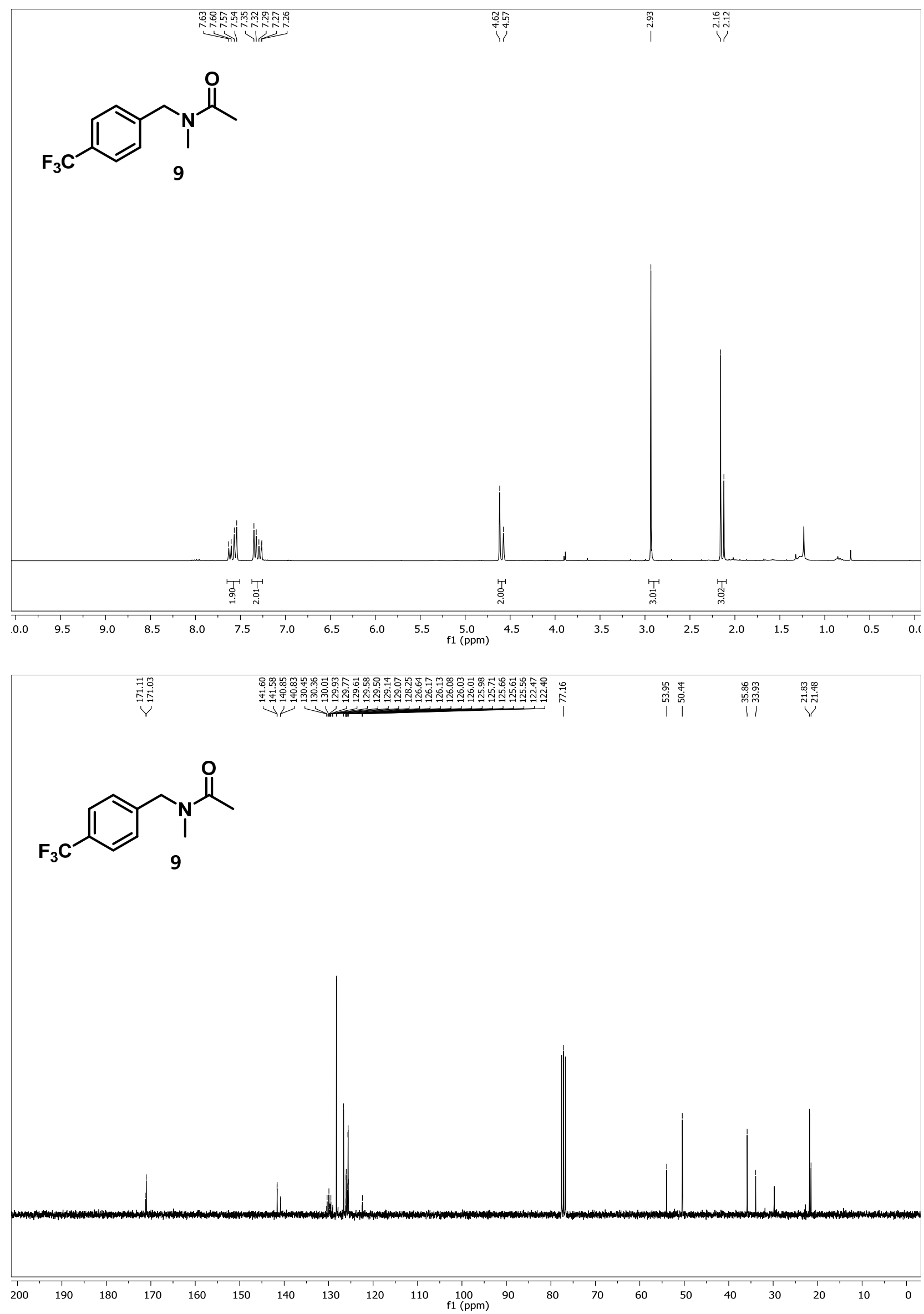

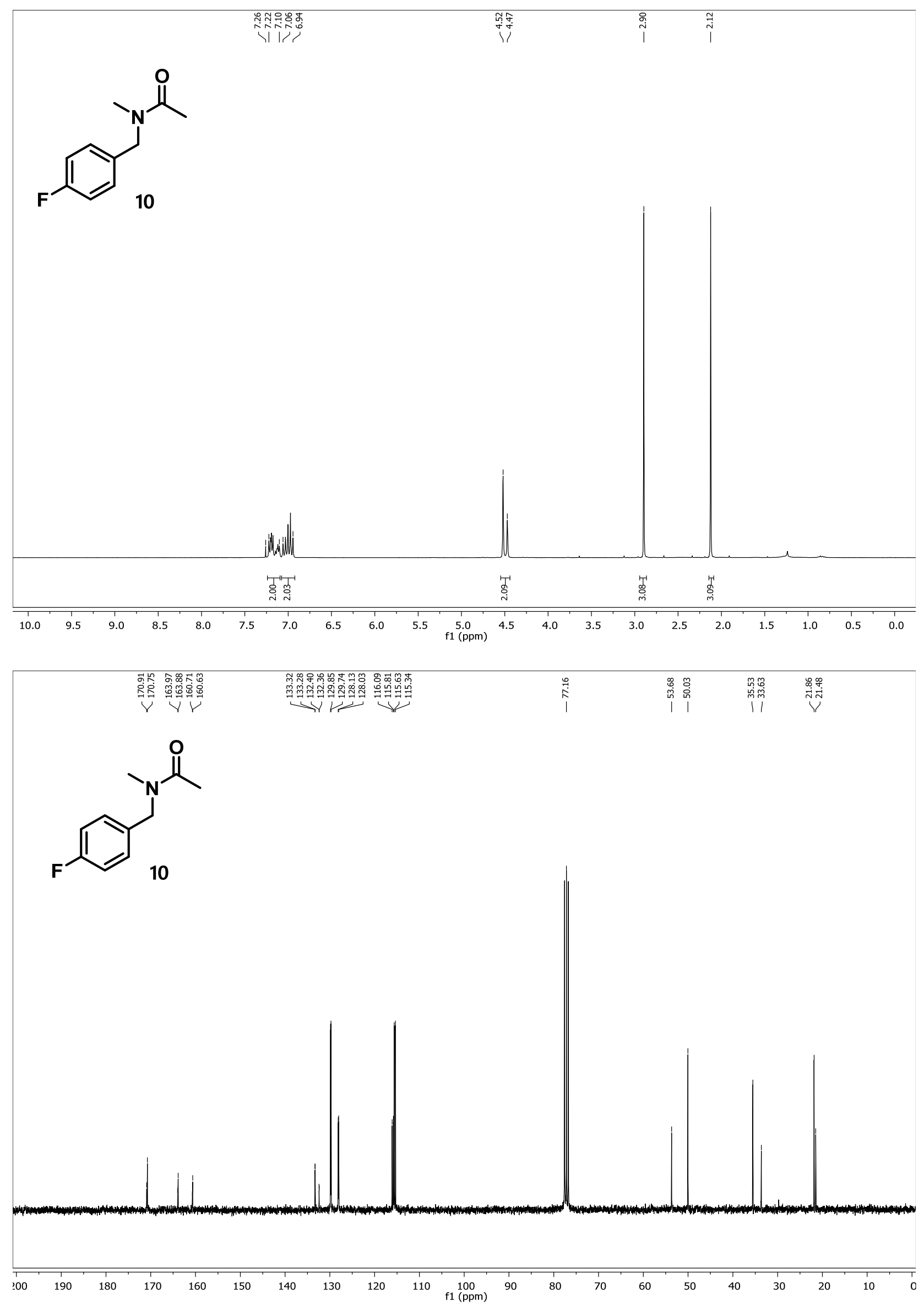

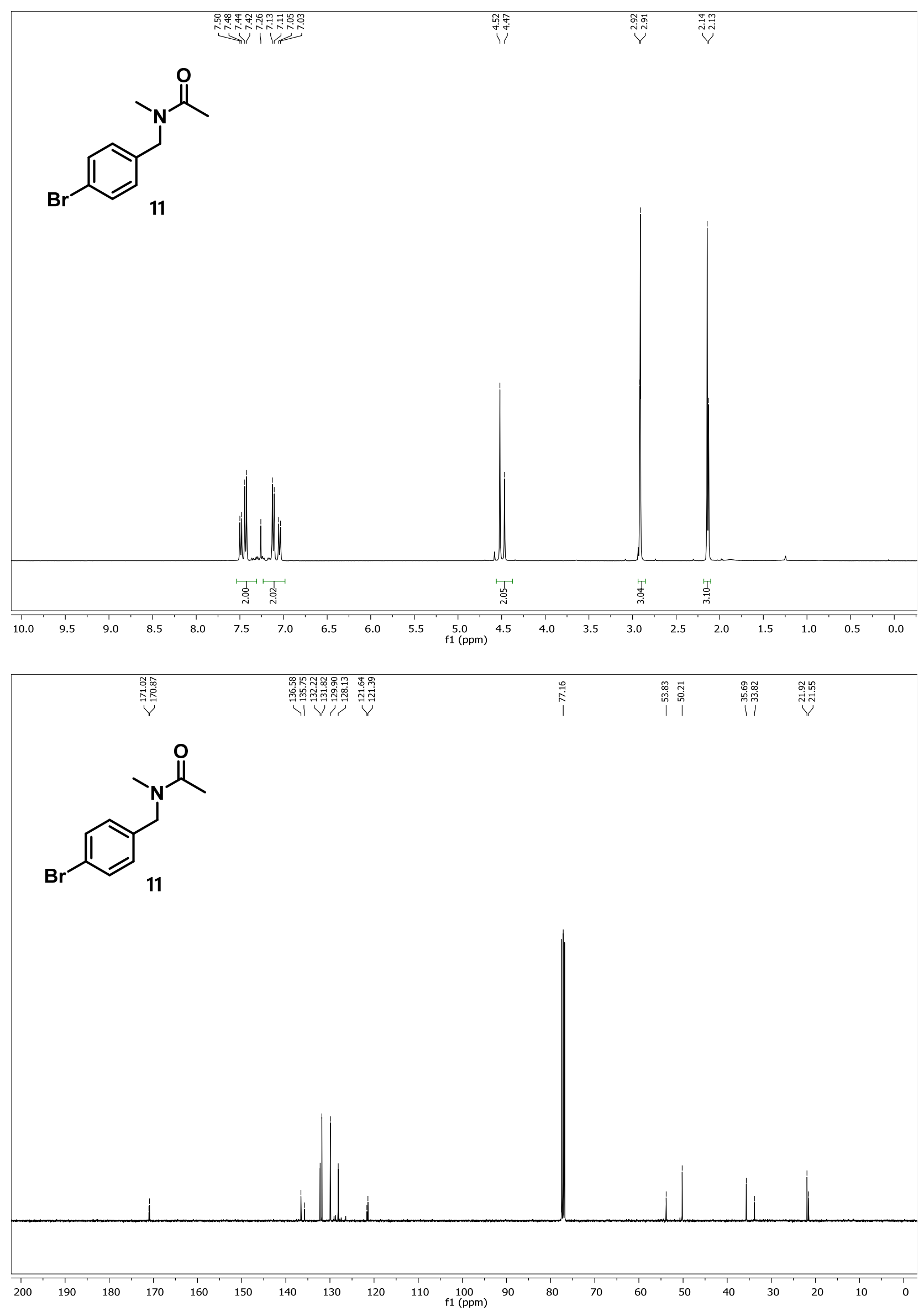

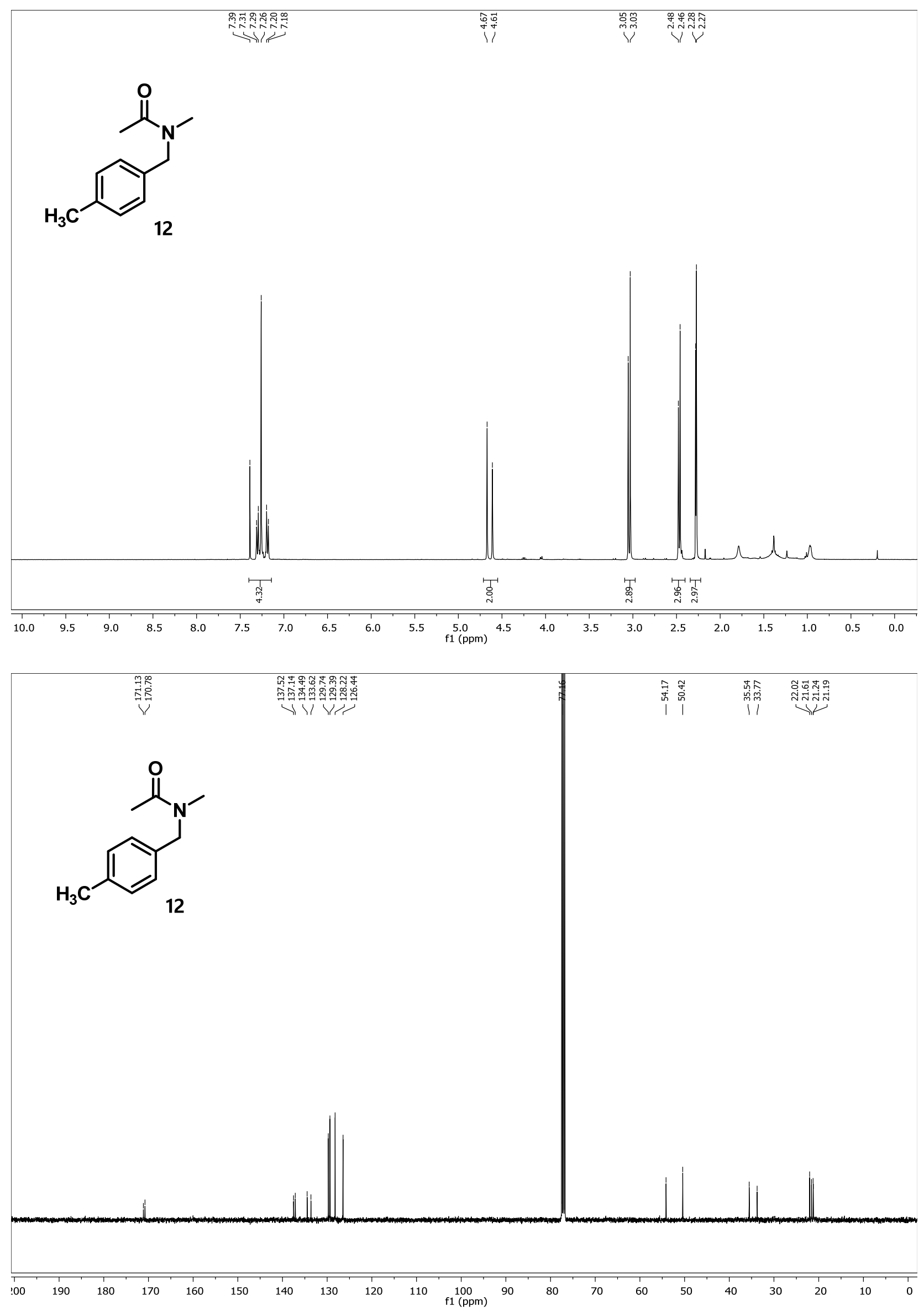

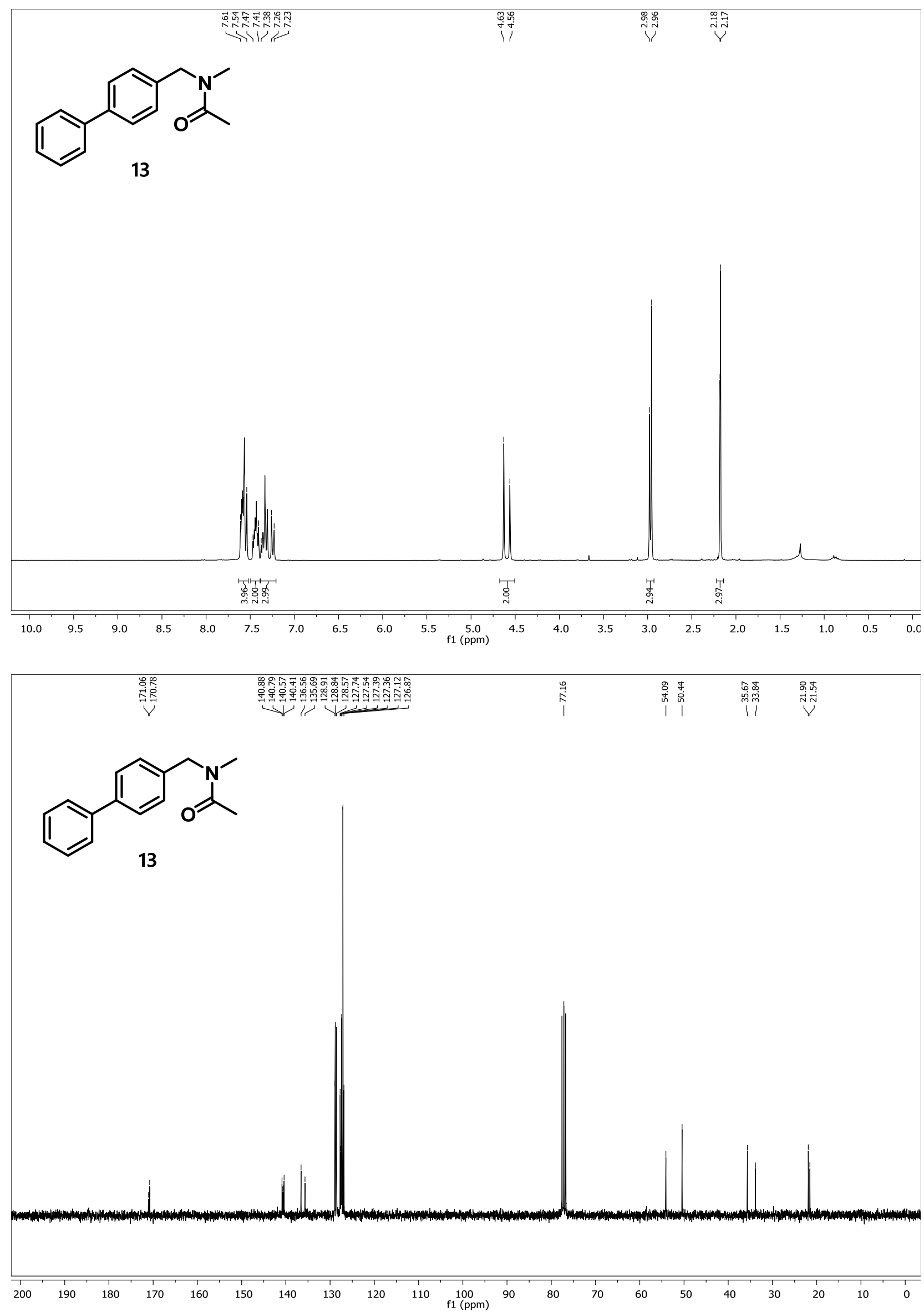

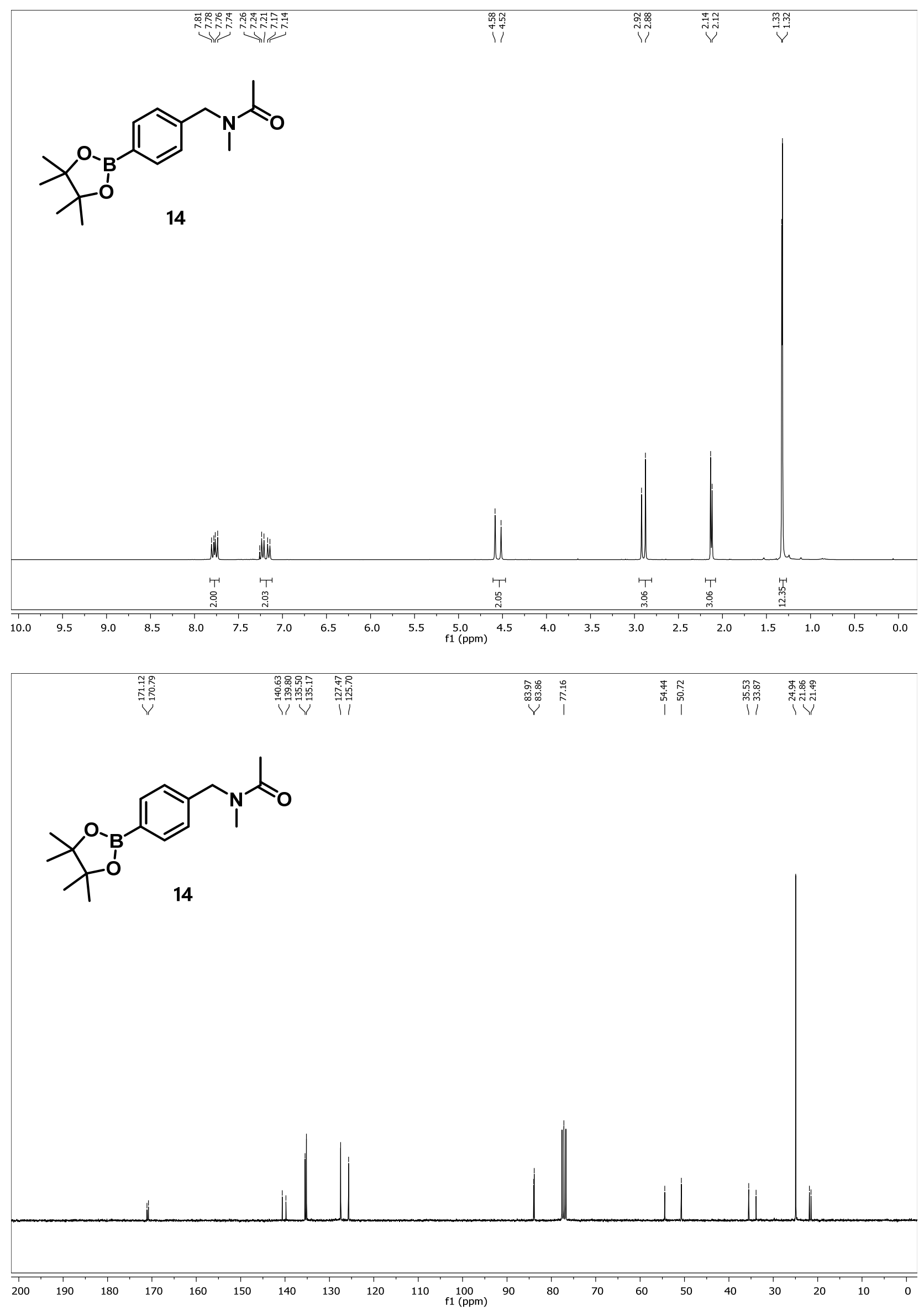

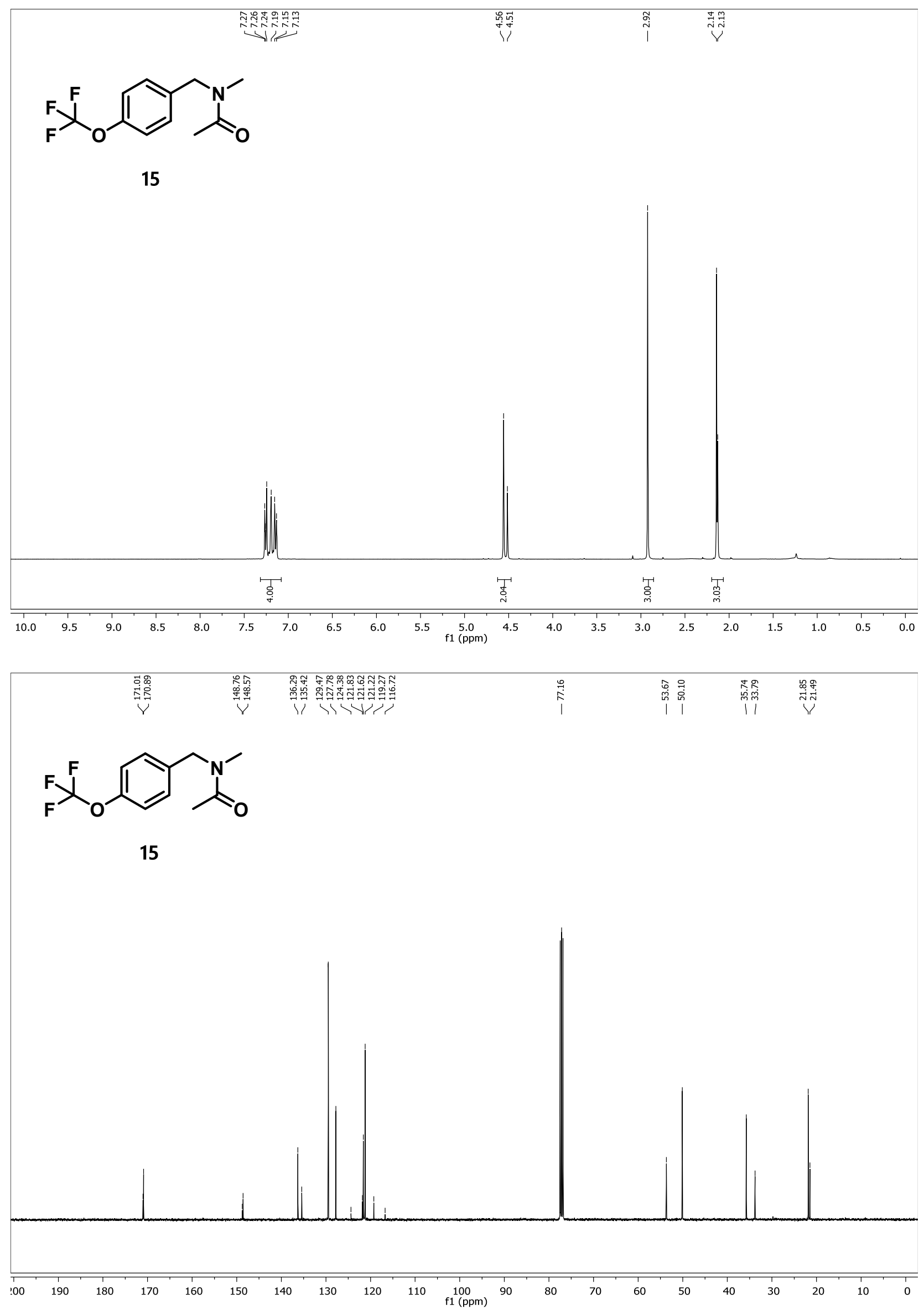

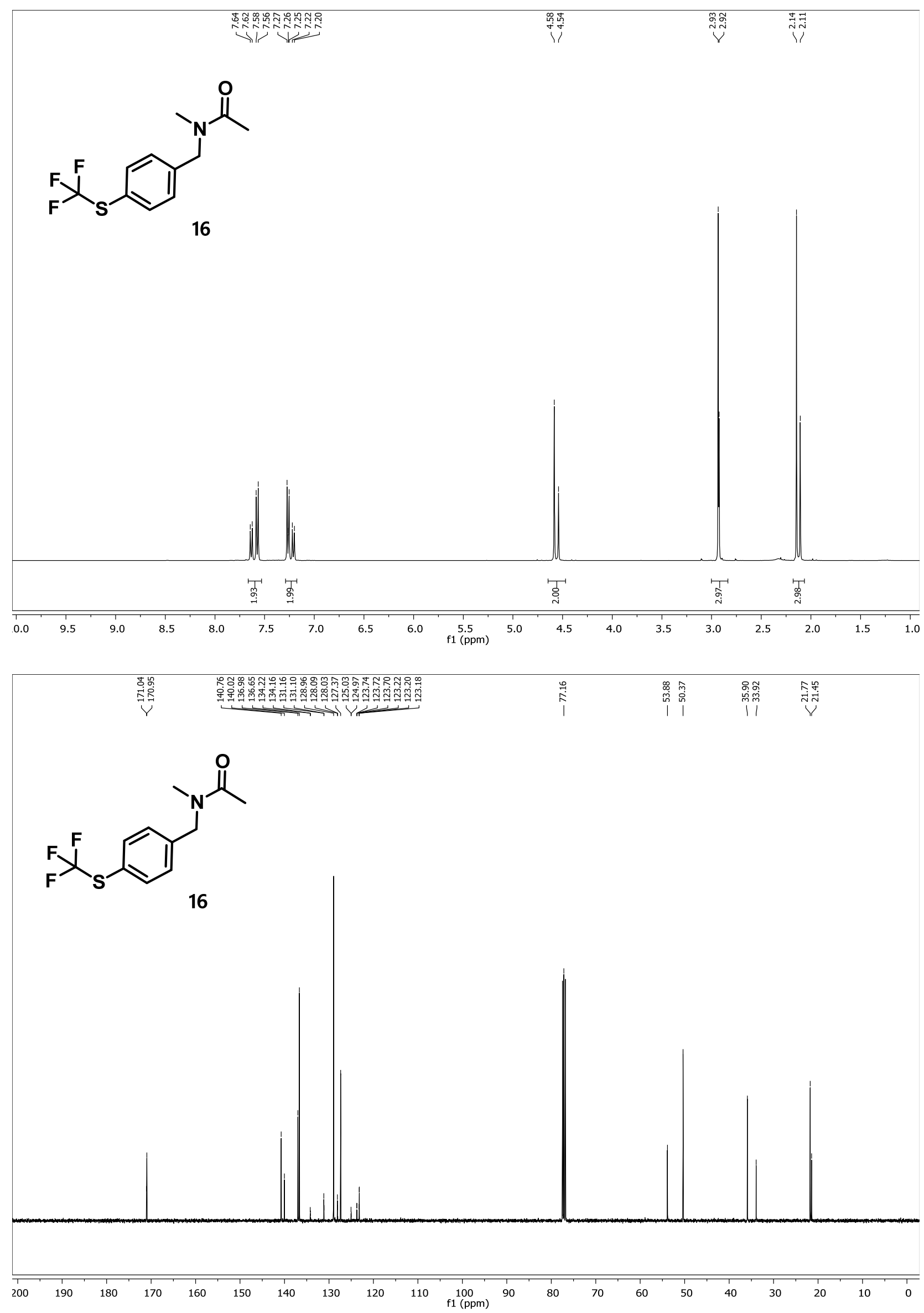

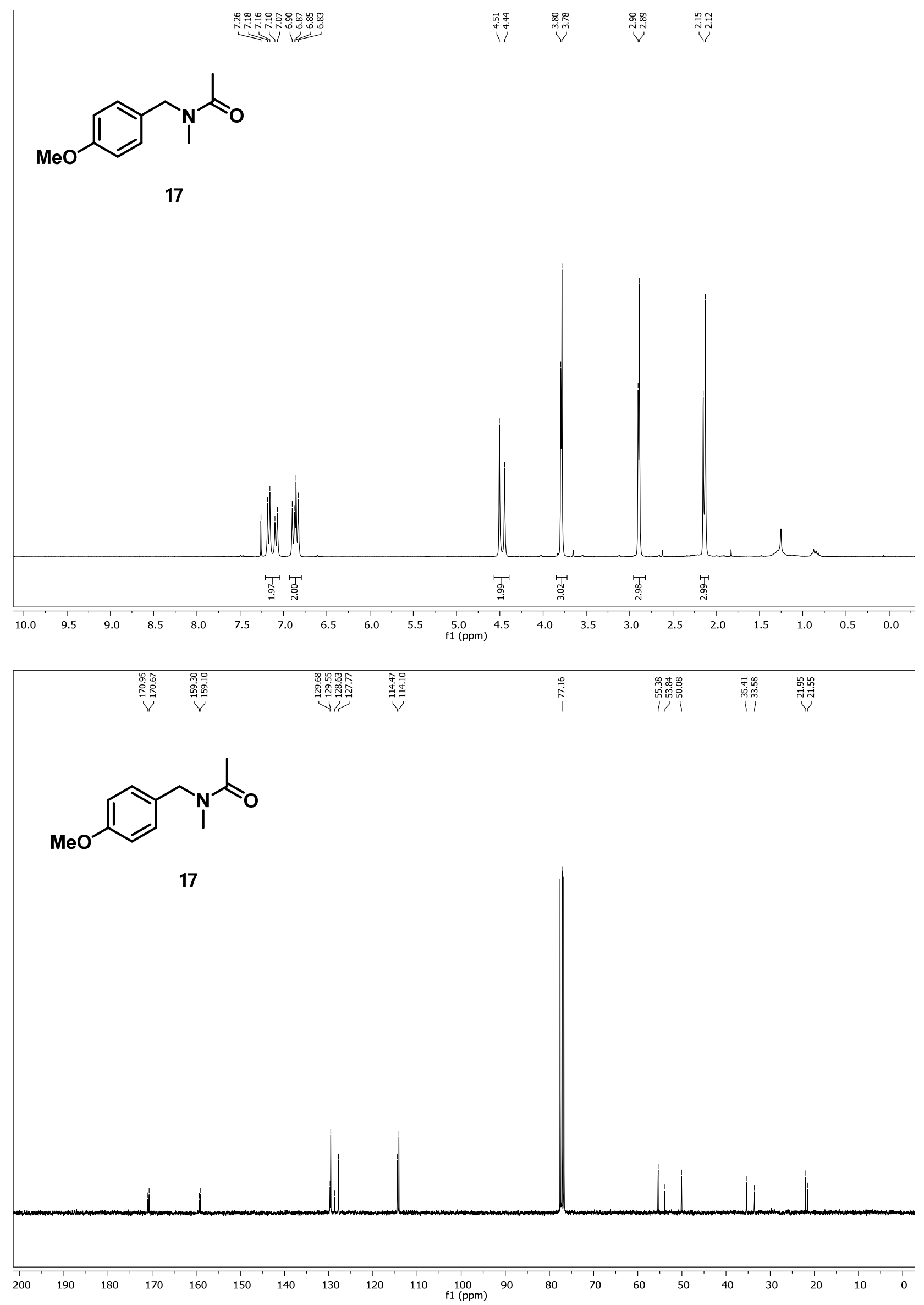

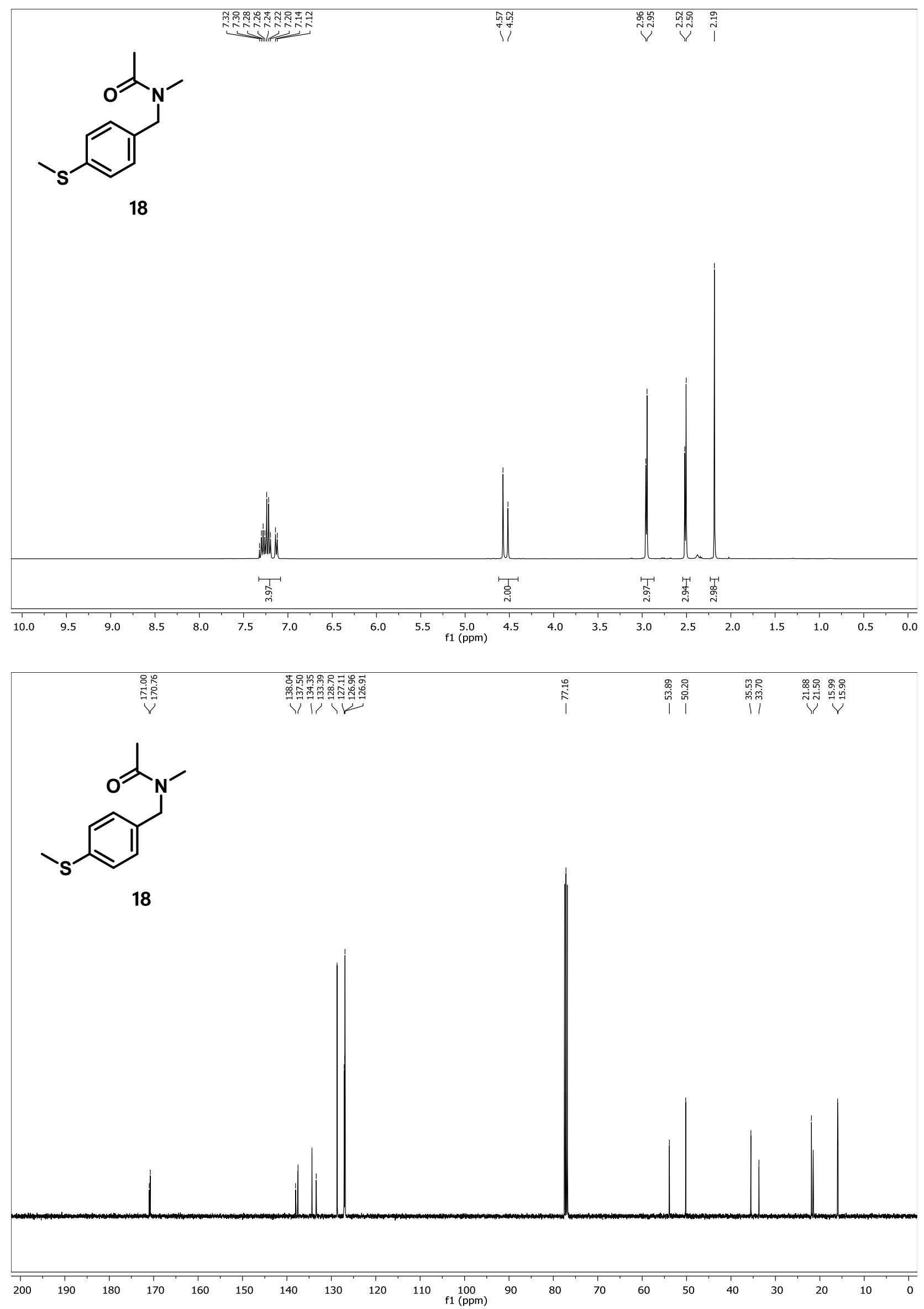

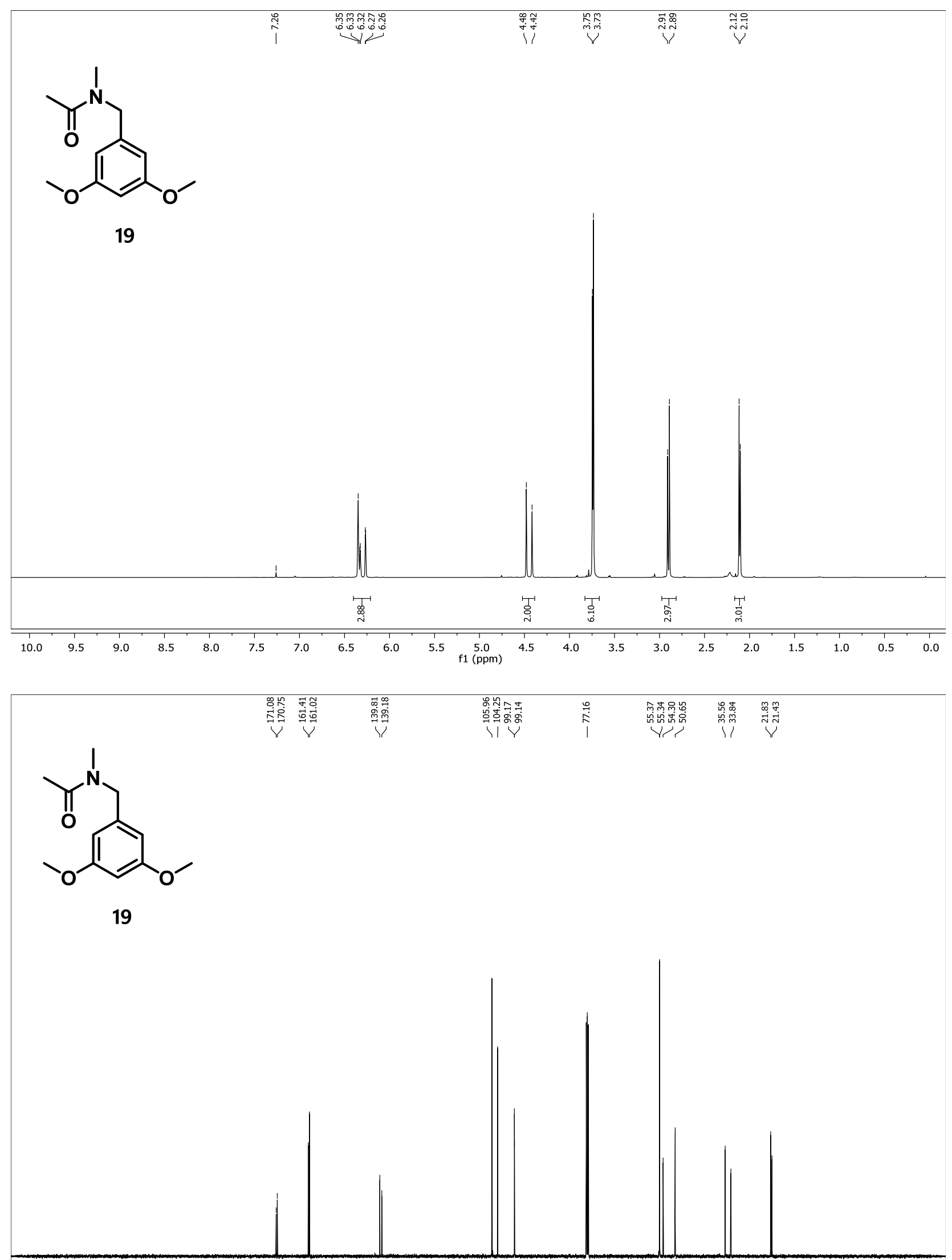

$\begin{array}{lllllllllllllllllllllllllllllll}550 & 240 & 230 & 220 & 210 & 200 & 190 & 180 & 170 & 160 & 150 & 140 & 130 & 120 & 110 & 100 & 90 & 80 & 70 & 60 & 50 & 40 & 30 & 20 & 10 & 0 & -10 & -20 & -3\end{array}$ 

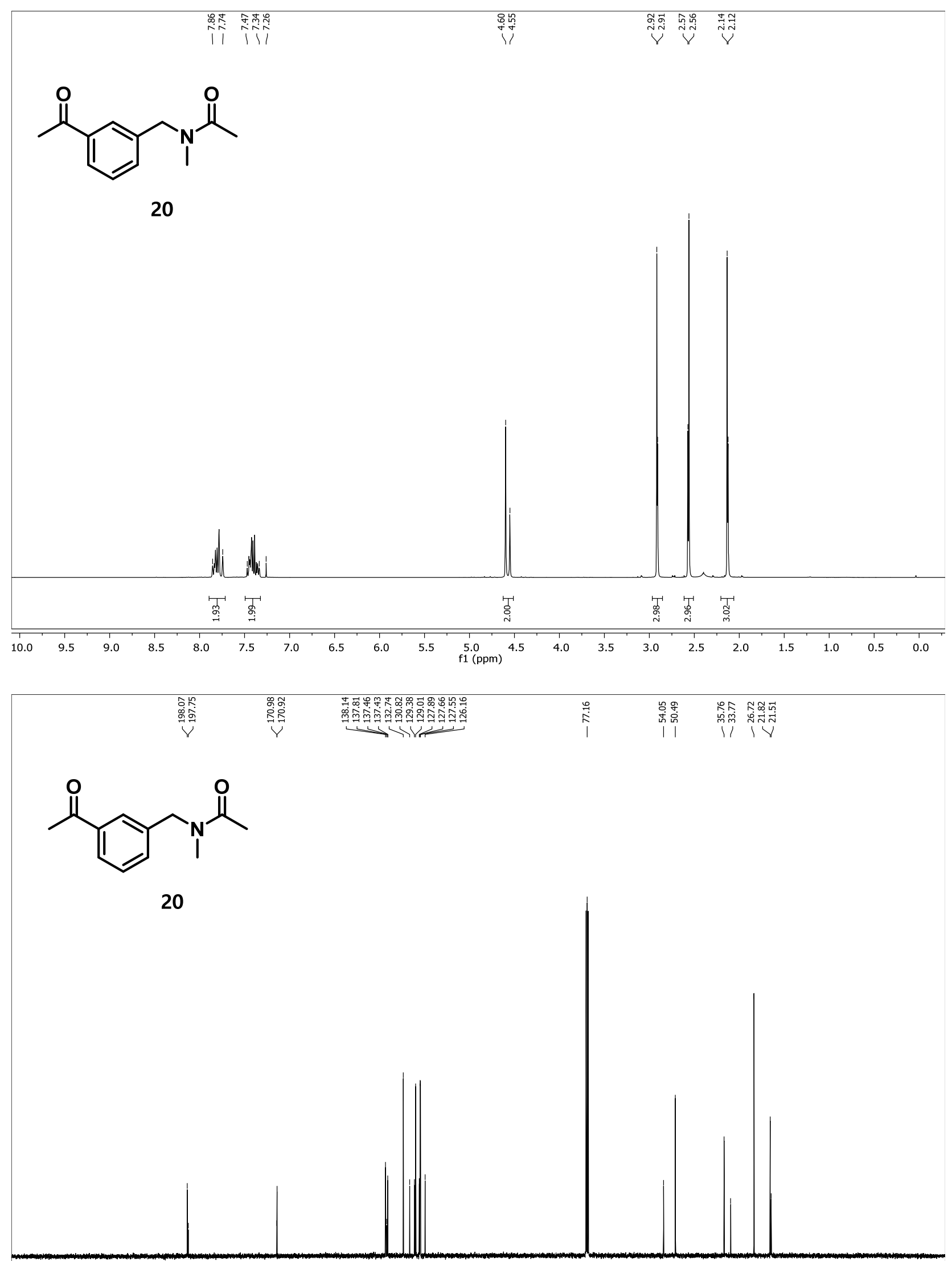

$\begin{array}{lllllllllllllllllllllllllllllll}250 & 240 & 230 & 220 & 210 & 200 & 190 & 180 & 170 & 160 & 150 & 140 & 130 & 120 & 110 & 100 & 90 & 80 & 70 & 60 & 50 & 40 & 30 & 20 & 10 & 0 & -10 & -20 & -3\end{array}$ 

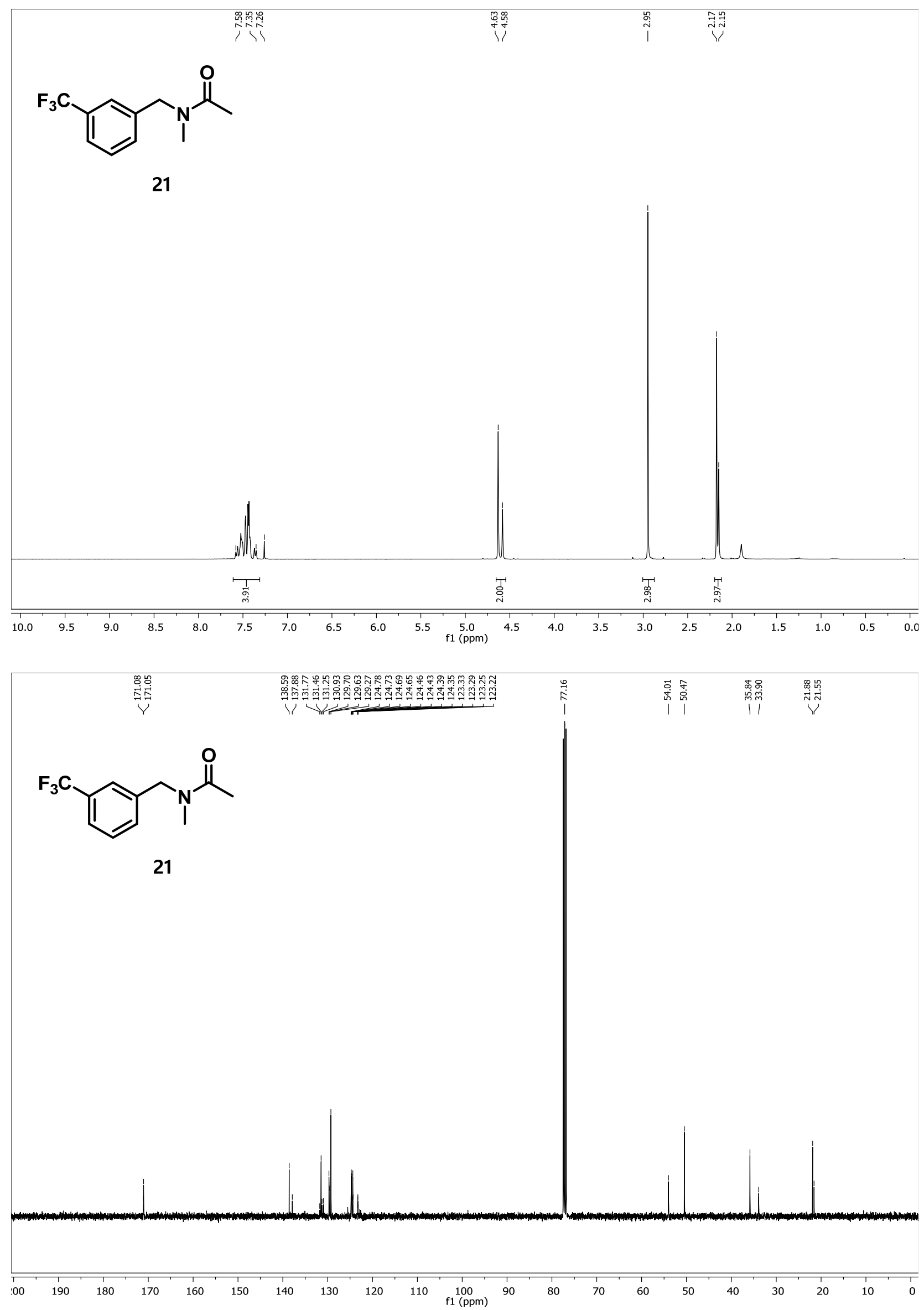

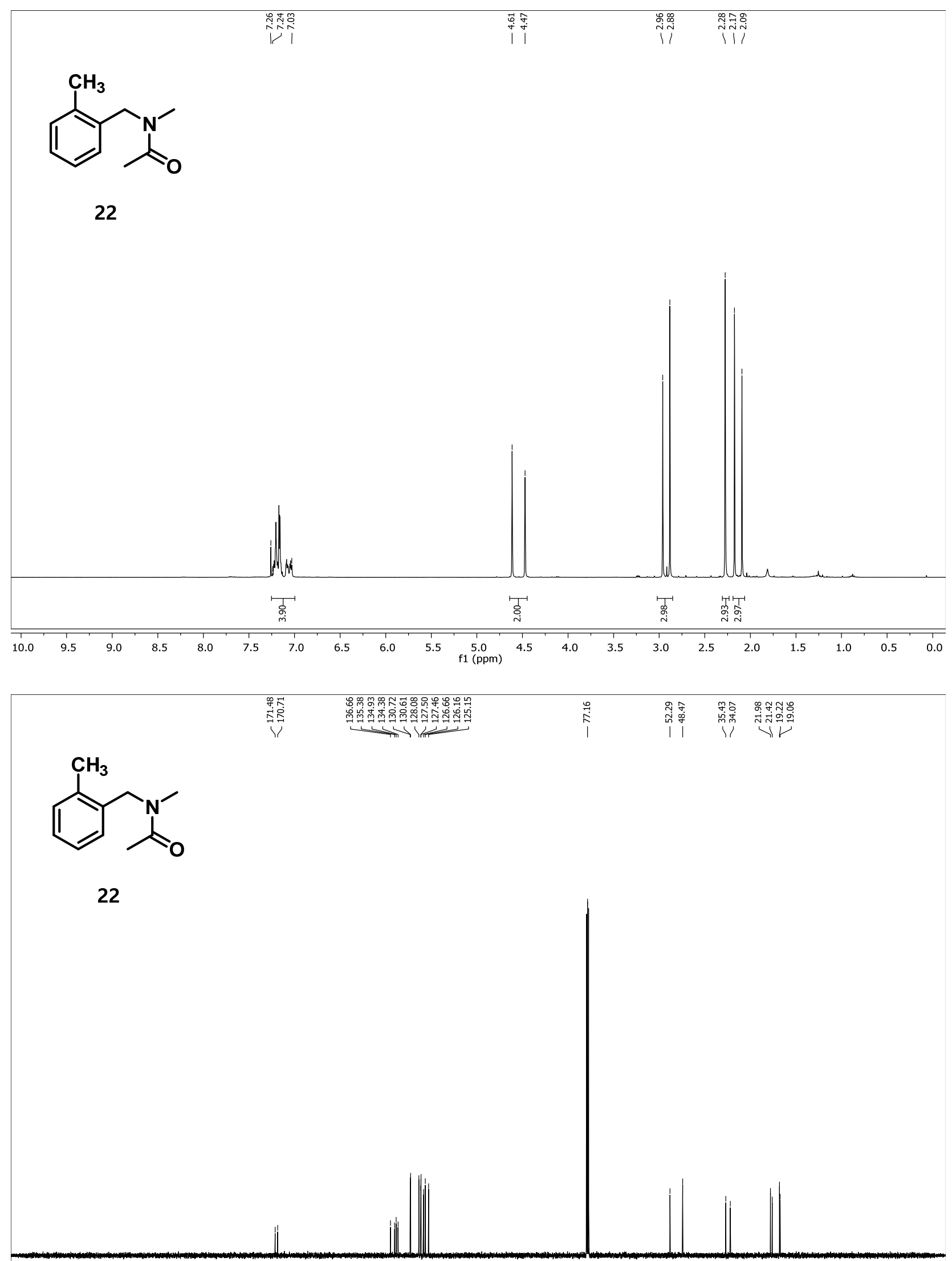

$\begin{array}{llllllllllllllllllllllllllllllllll}250 & 240 & 230 & 220 & 210 & 200 & 190 & 180 & 170 & 160 & 150 & 140 & 130 & 120 & 110 & 100 & 90 & 80 & 70 & 60 & 50 & 40 & 30 & 20 & 10 & 0 & -10 & -20 & -3\end{array}$ 

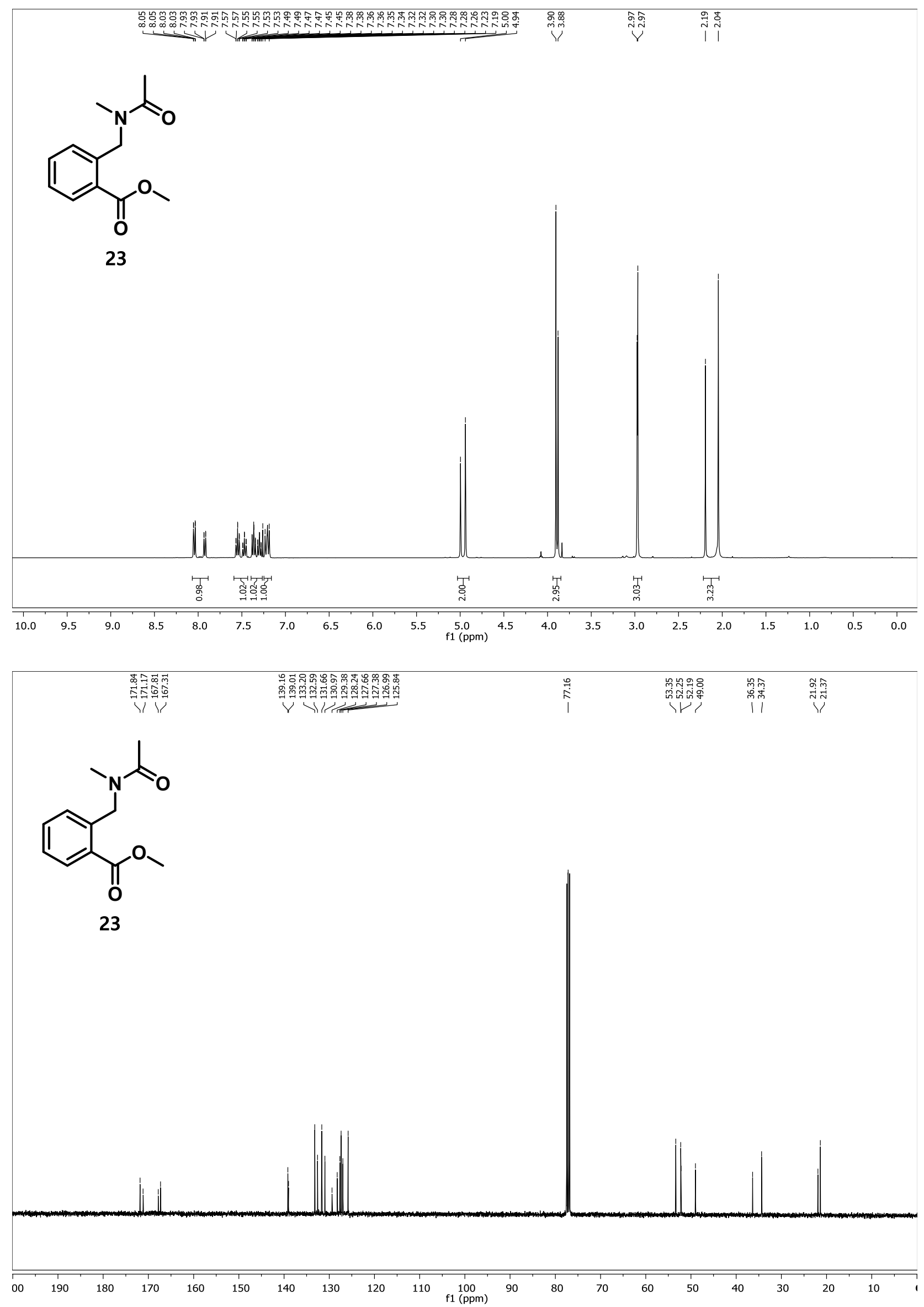

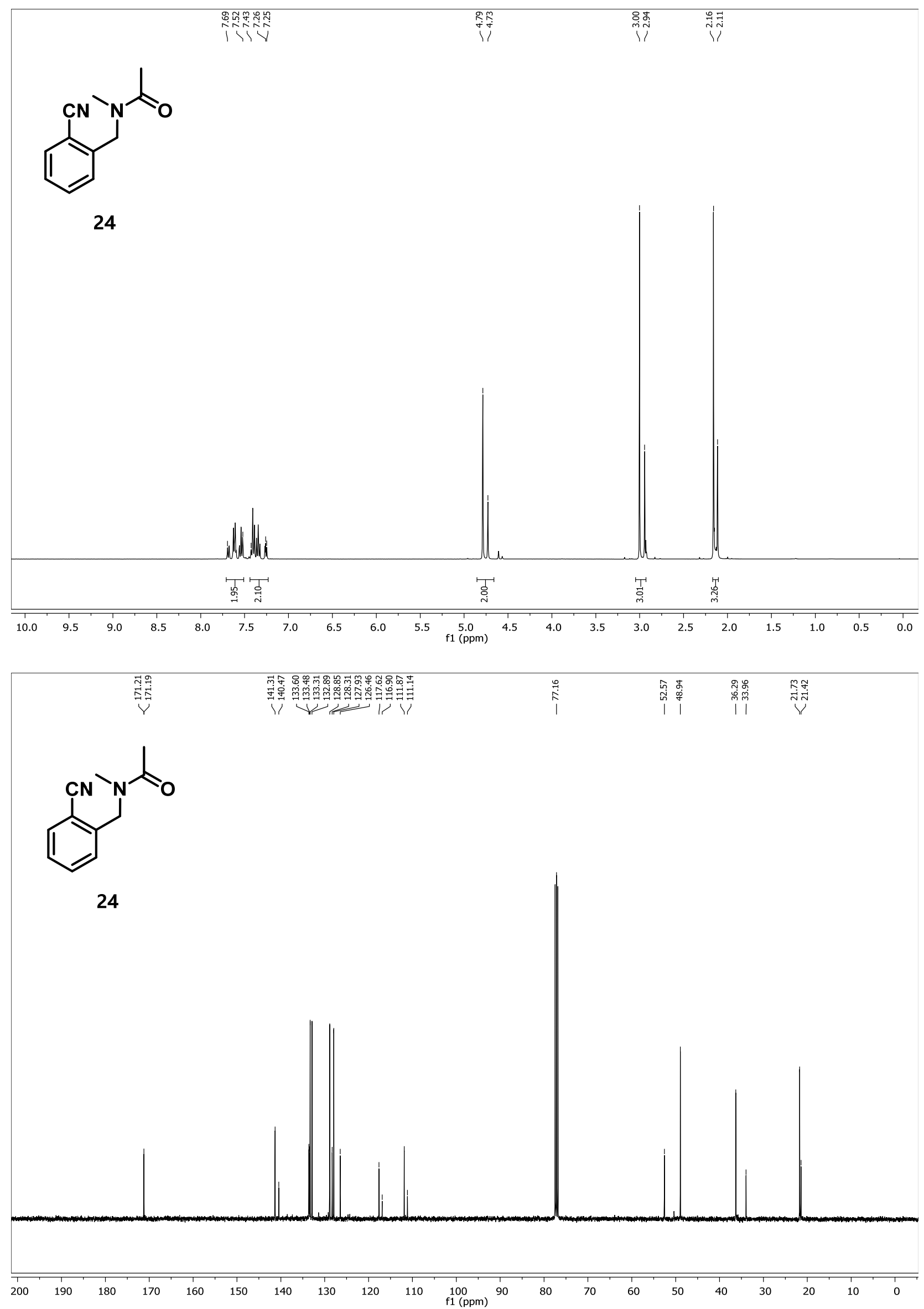

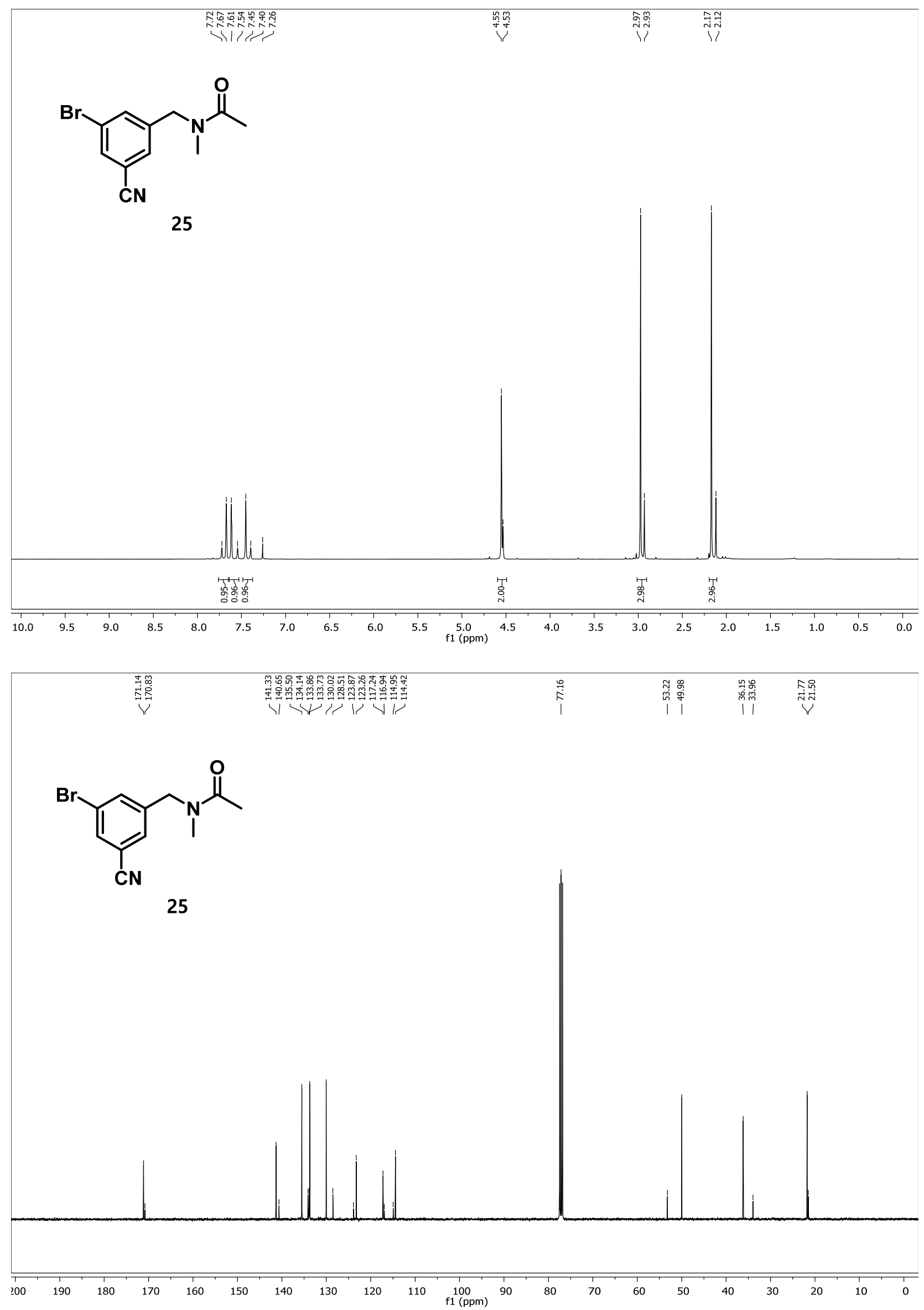

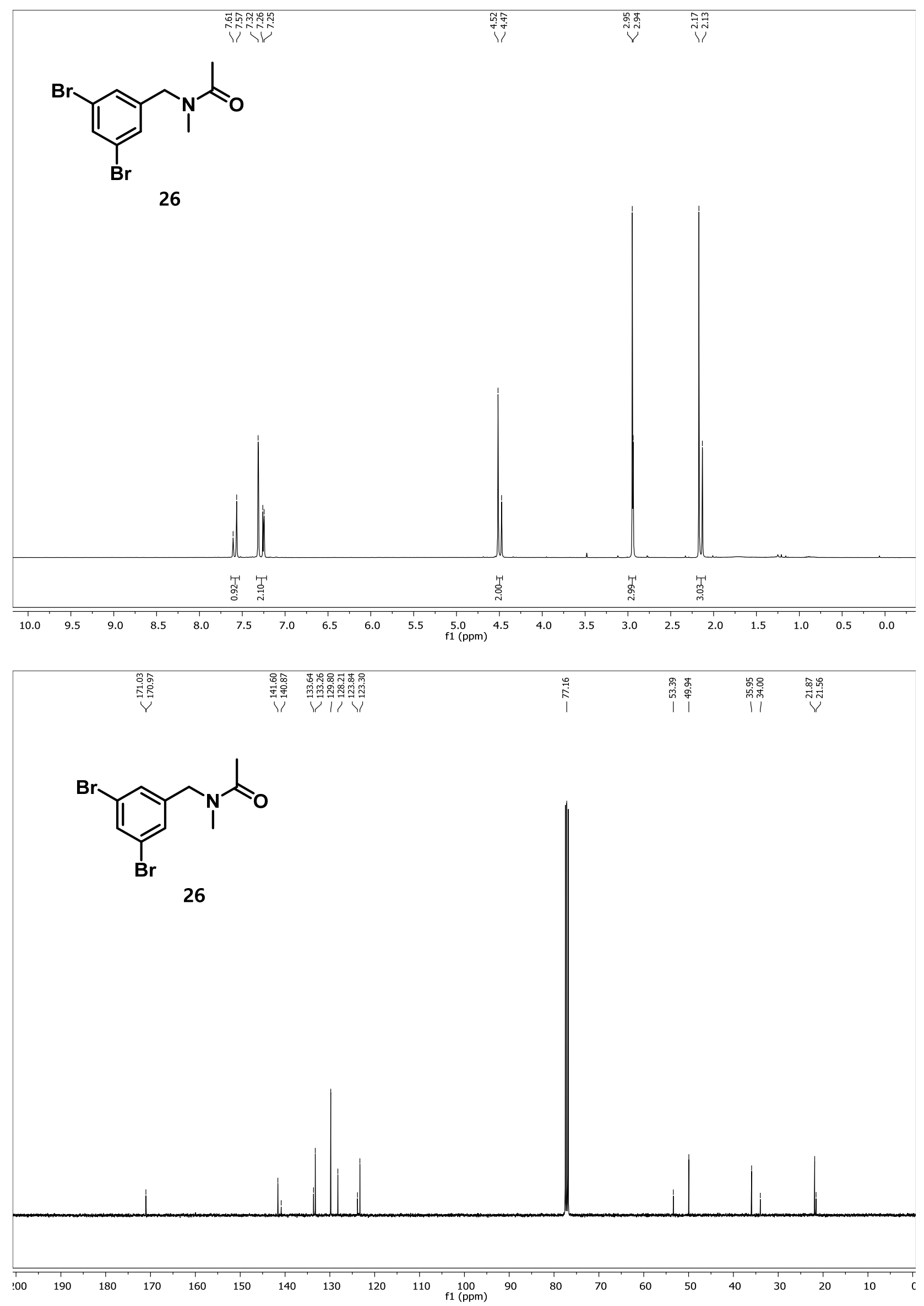

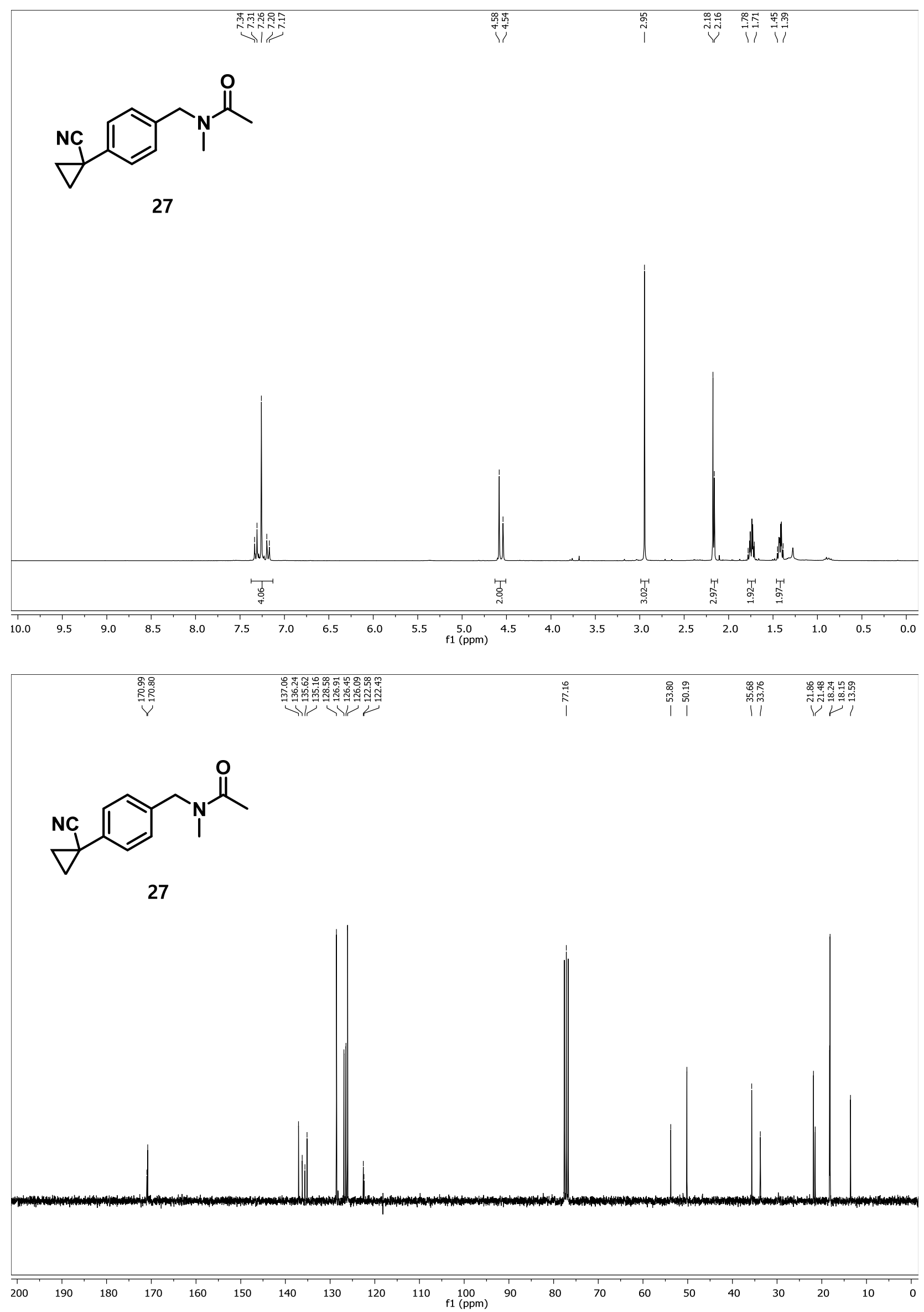

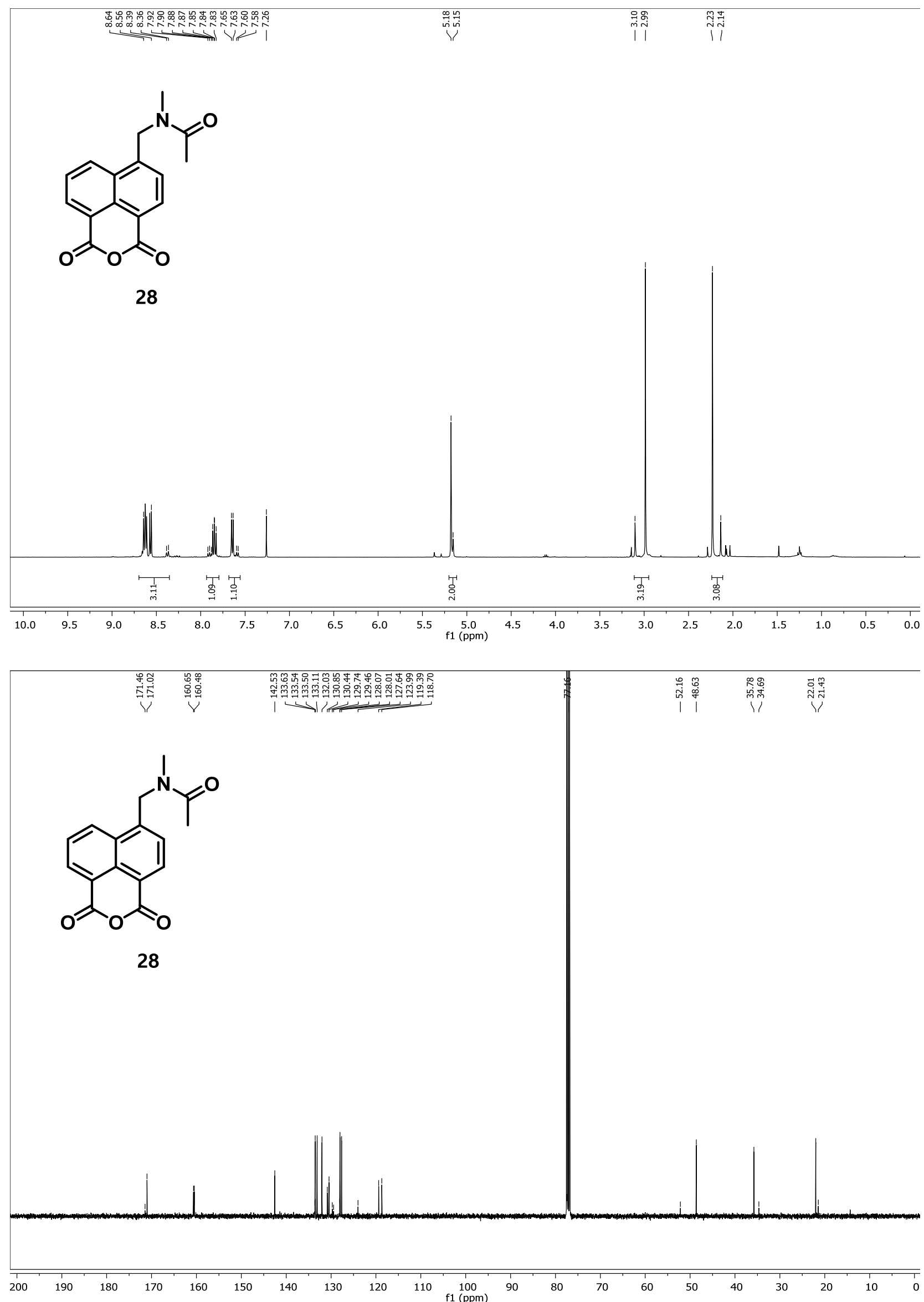

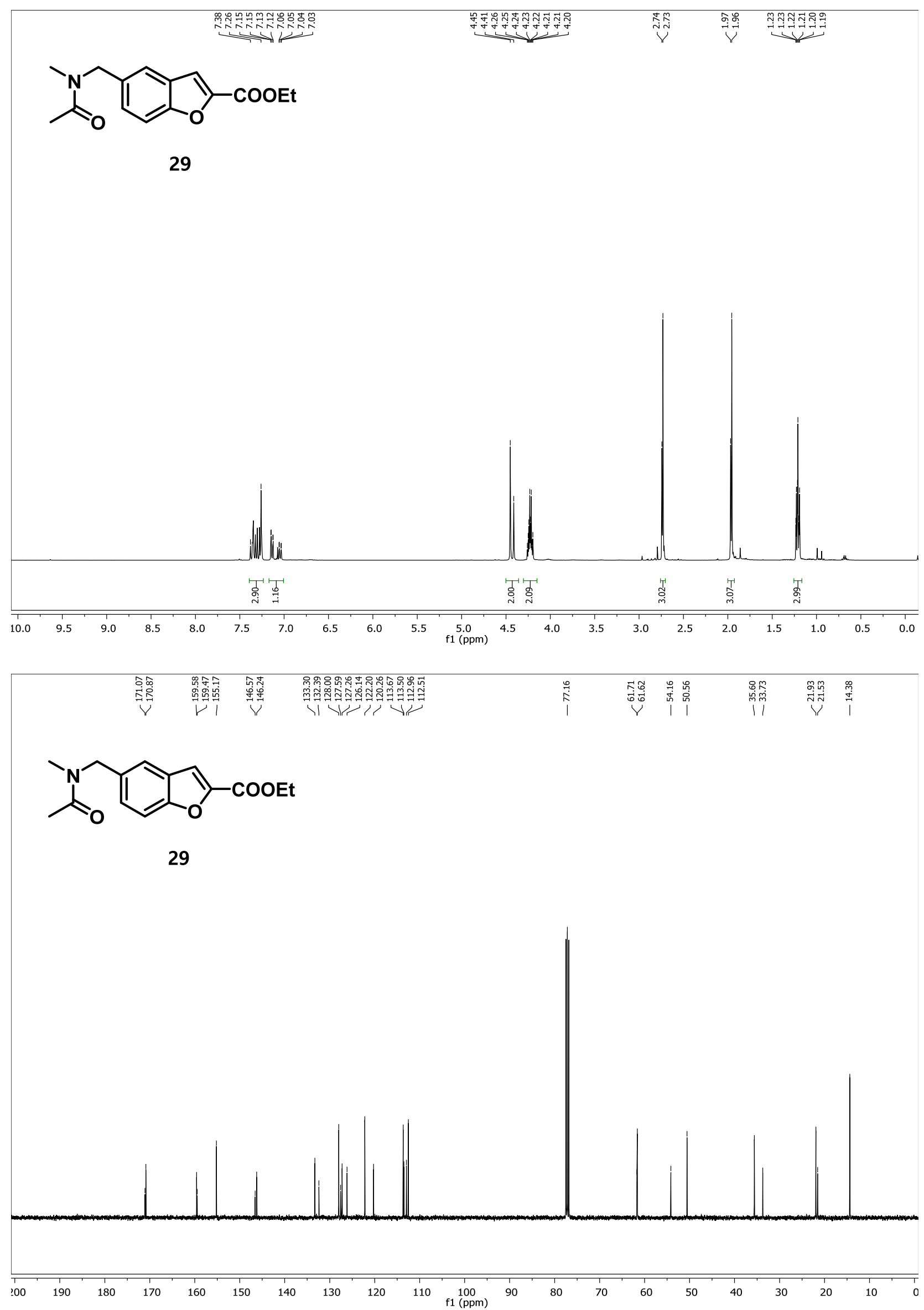

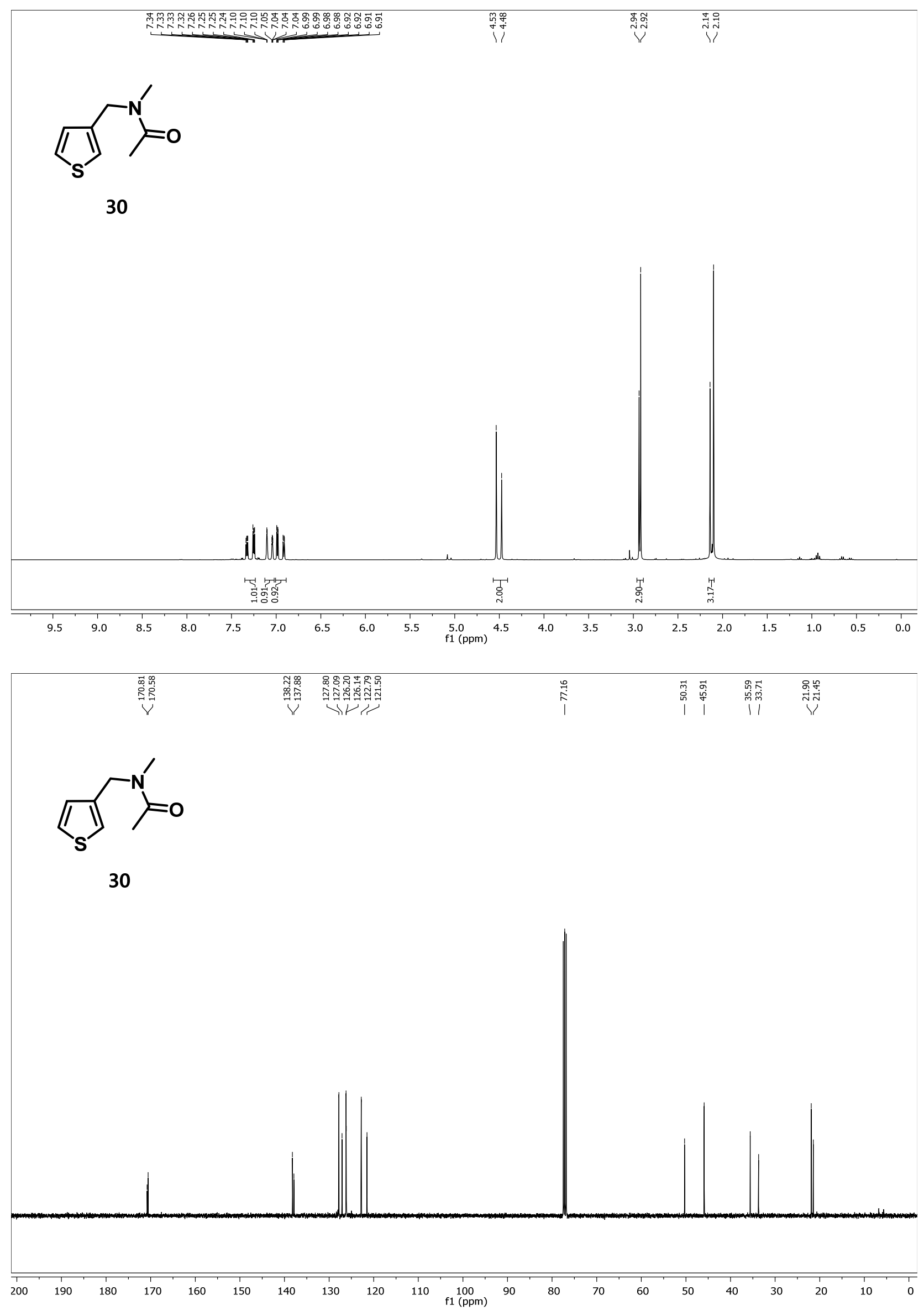

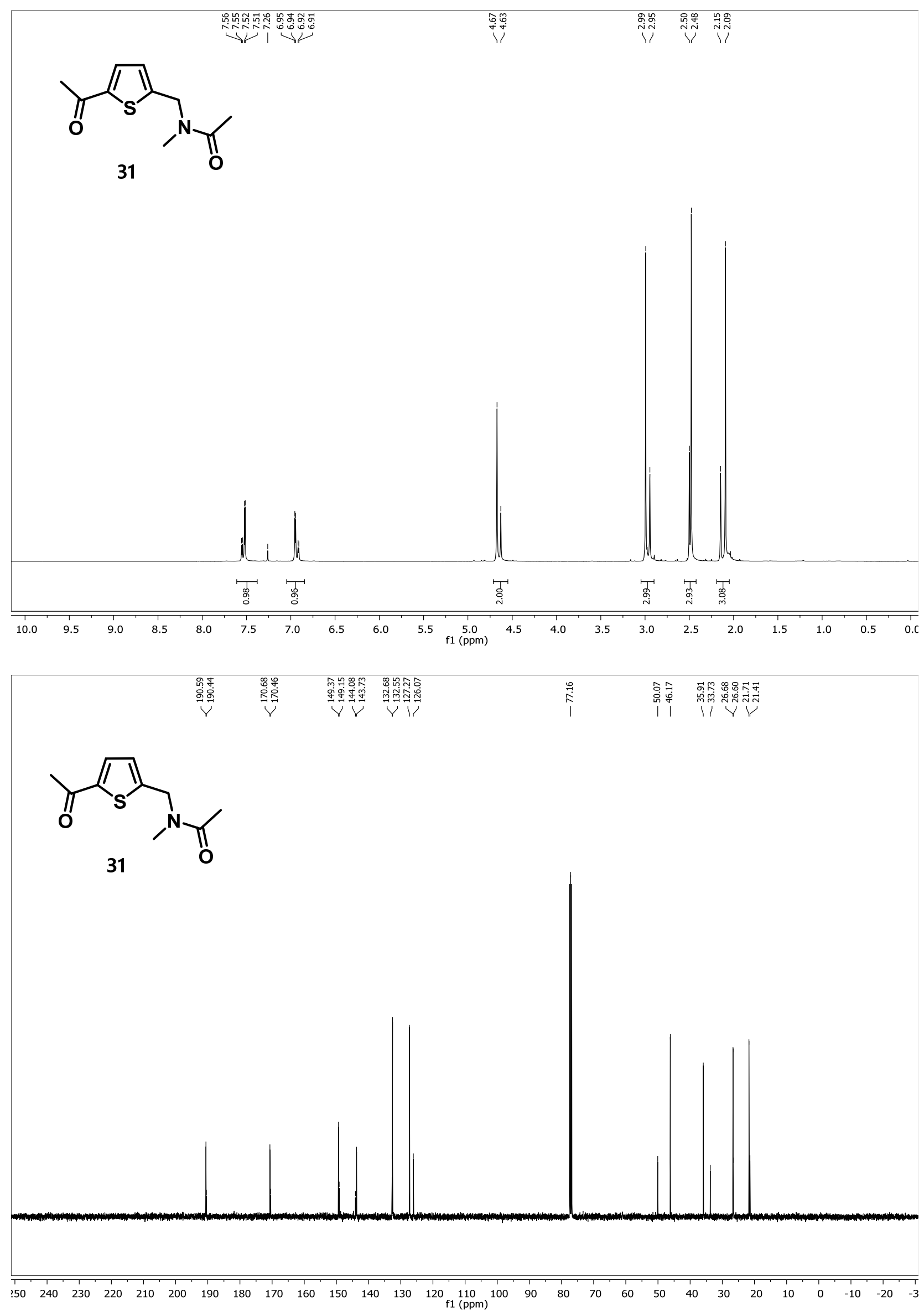

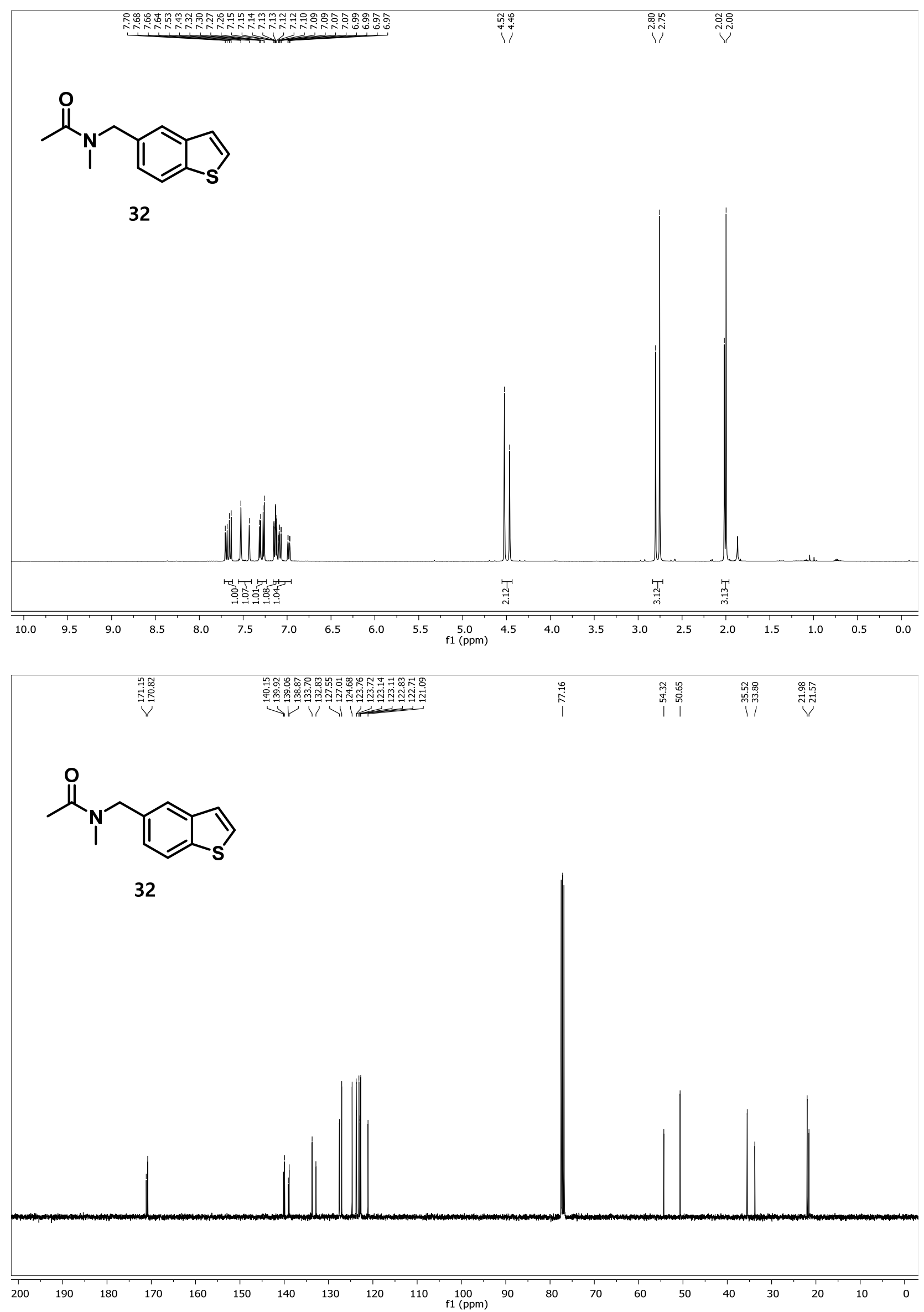

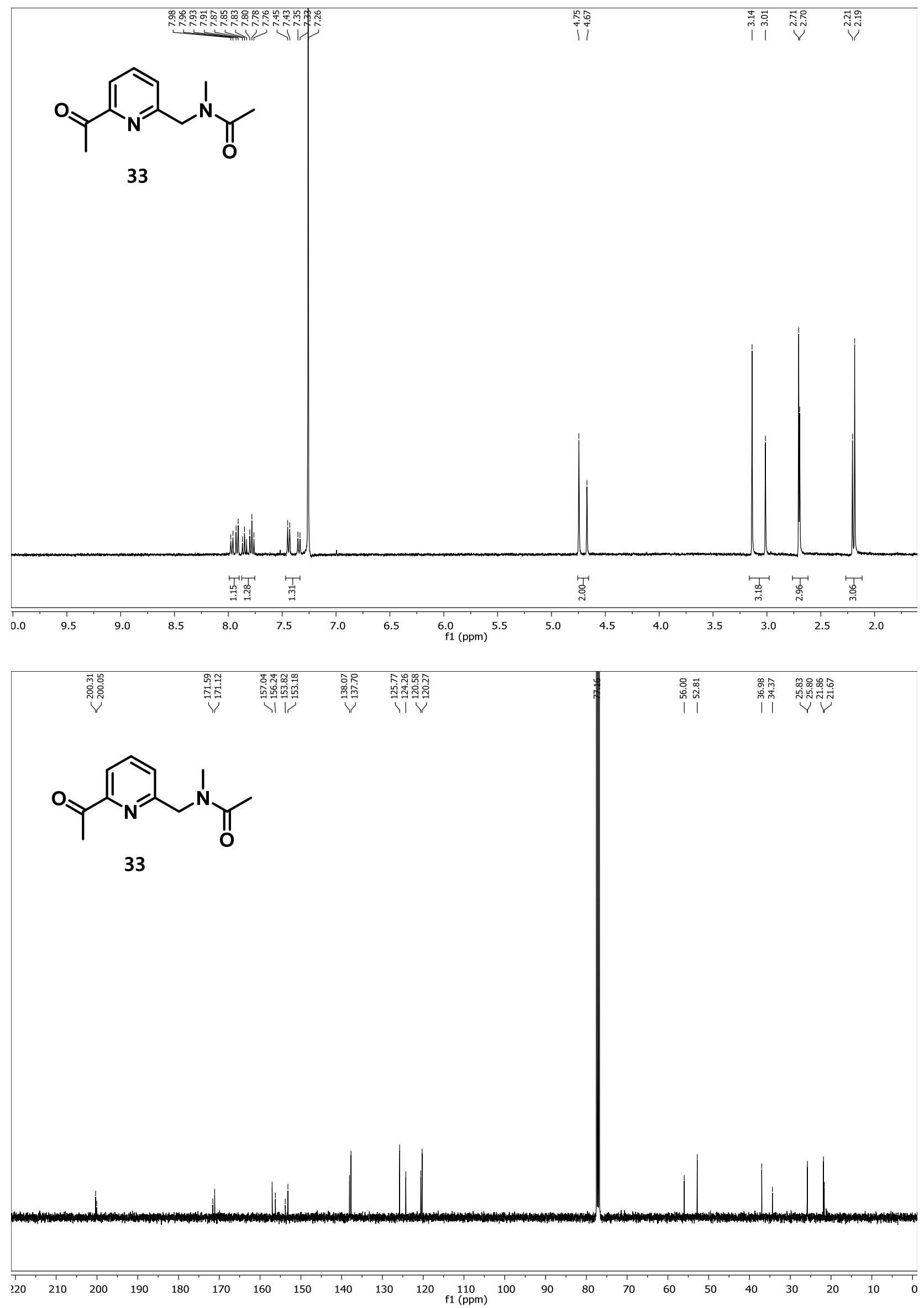

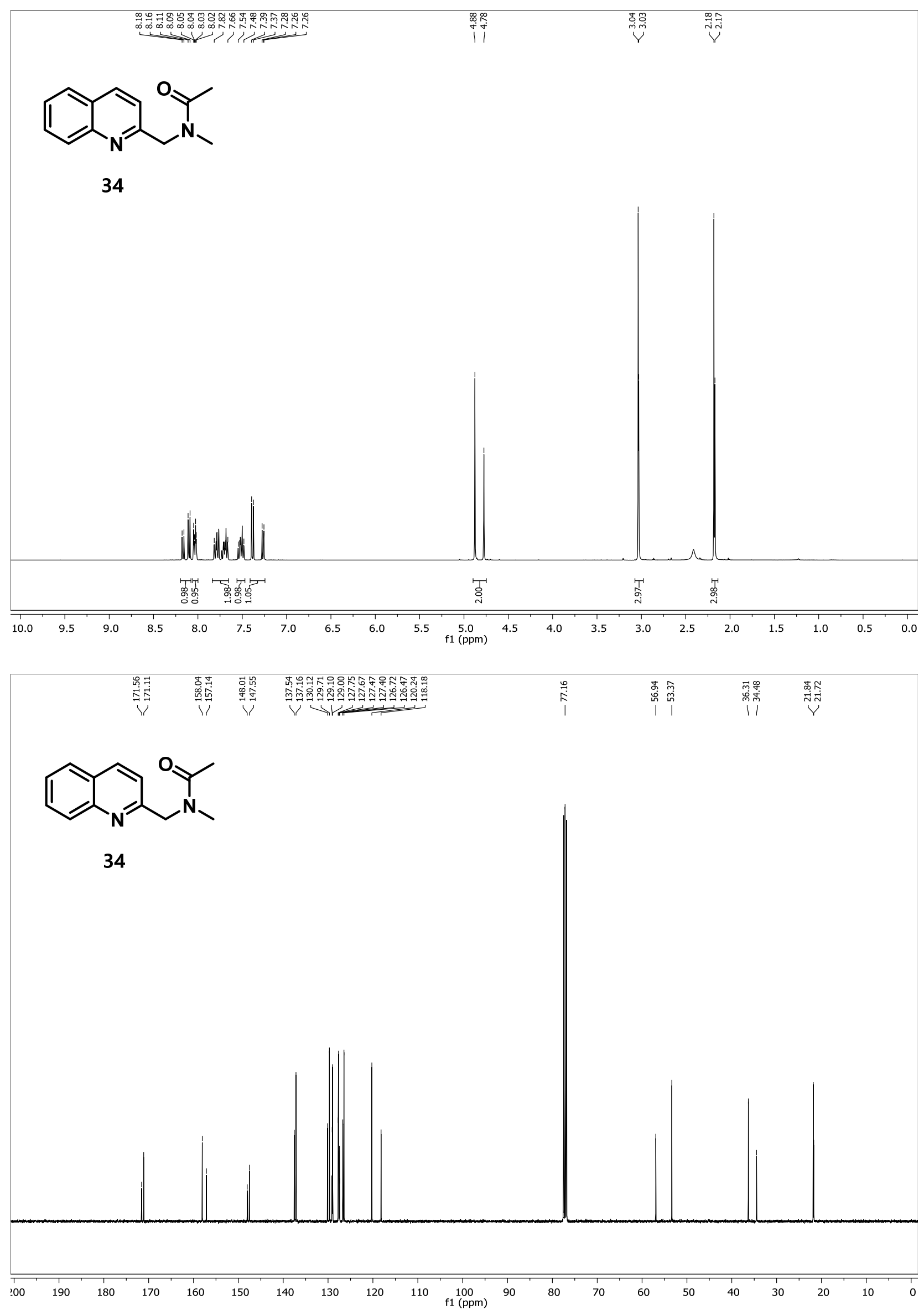

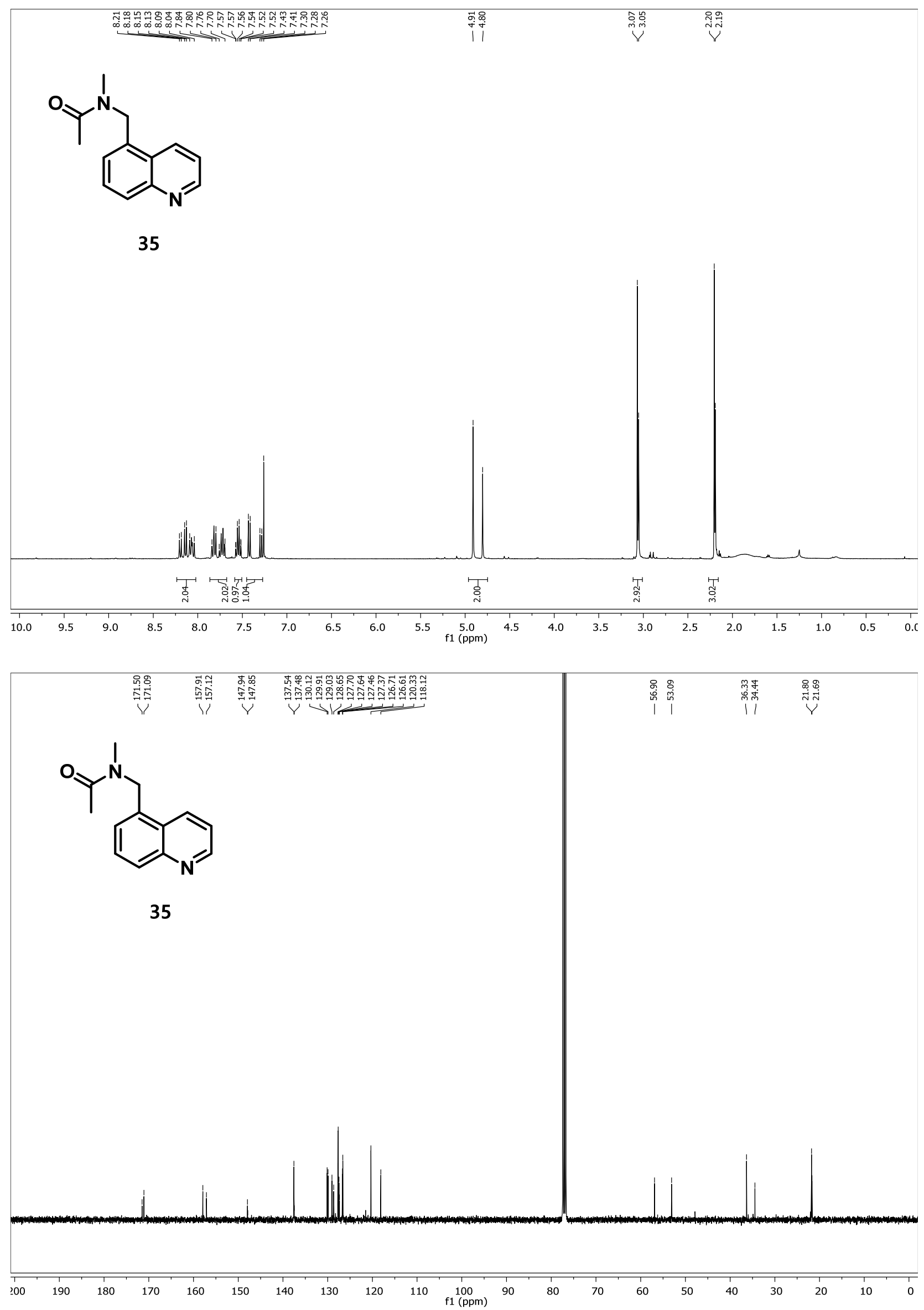

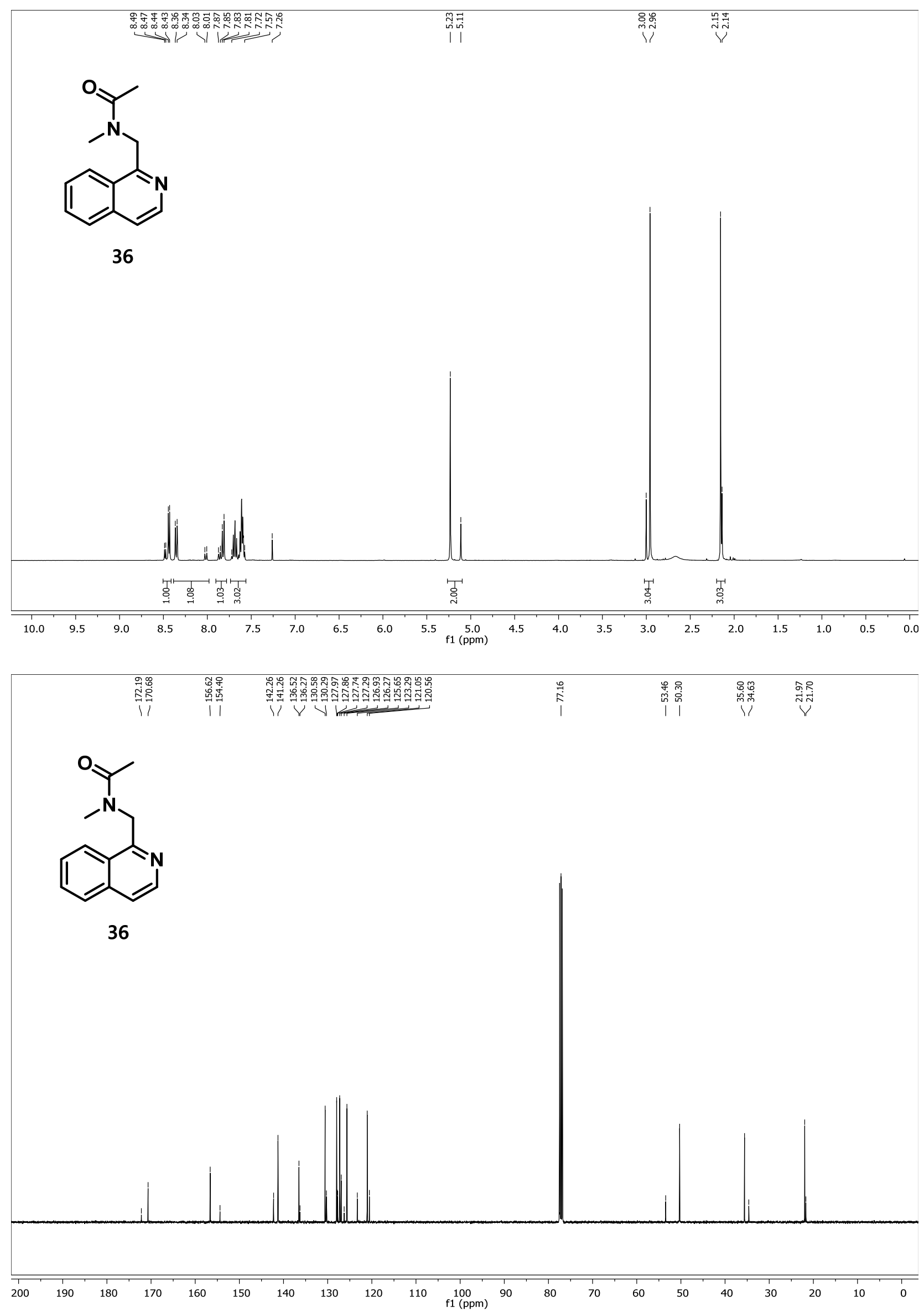

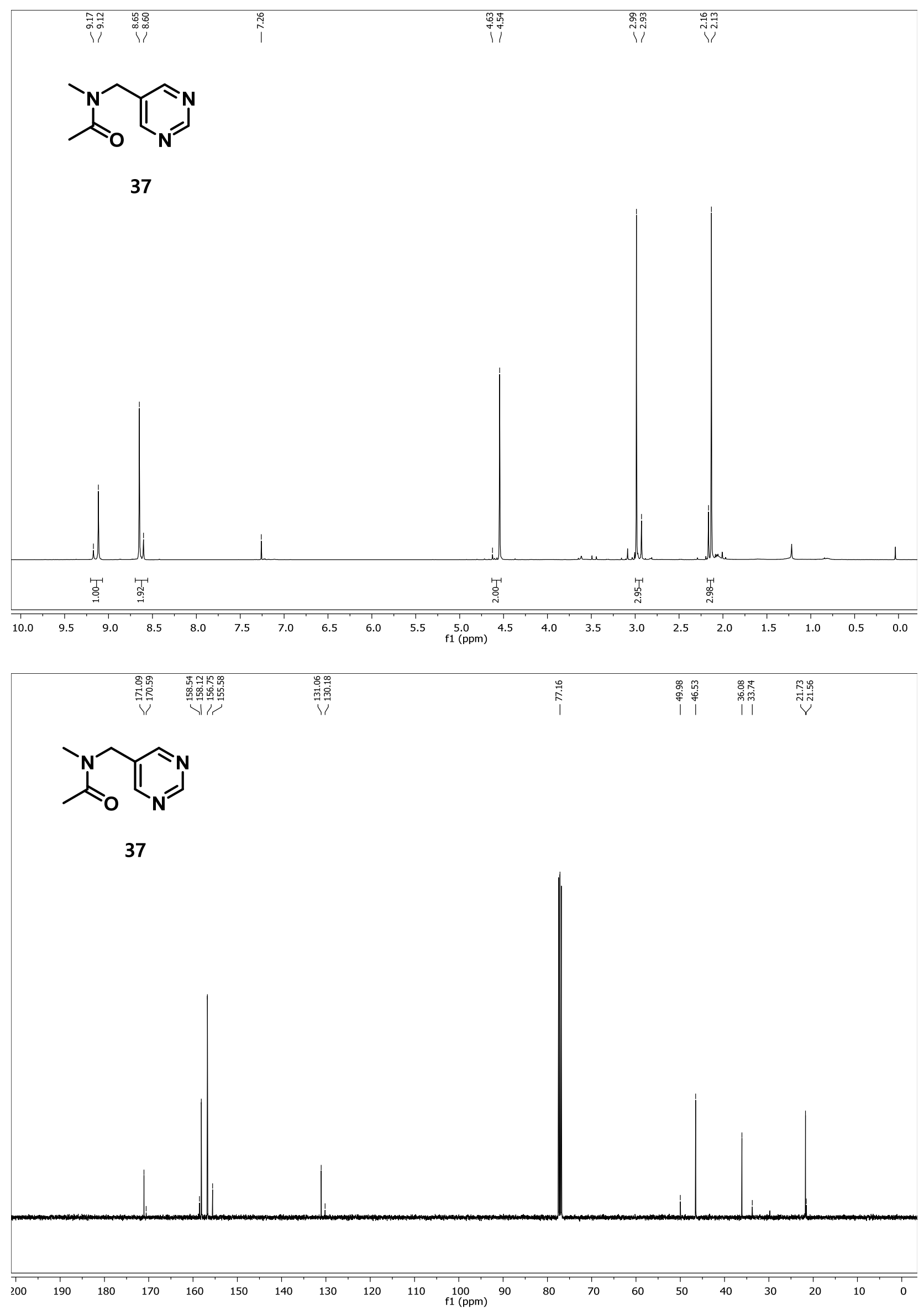

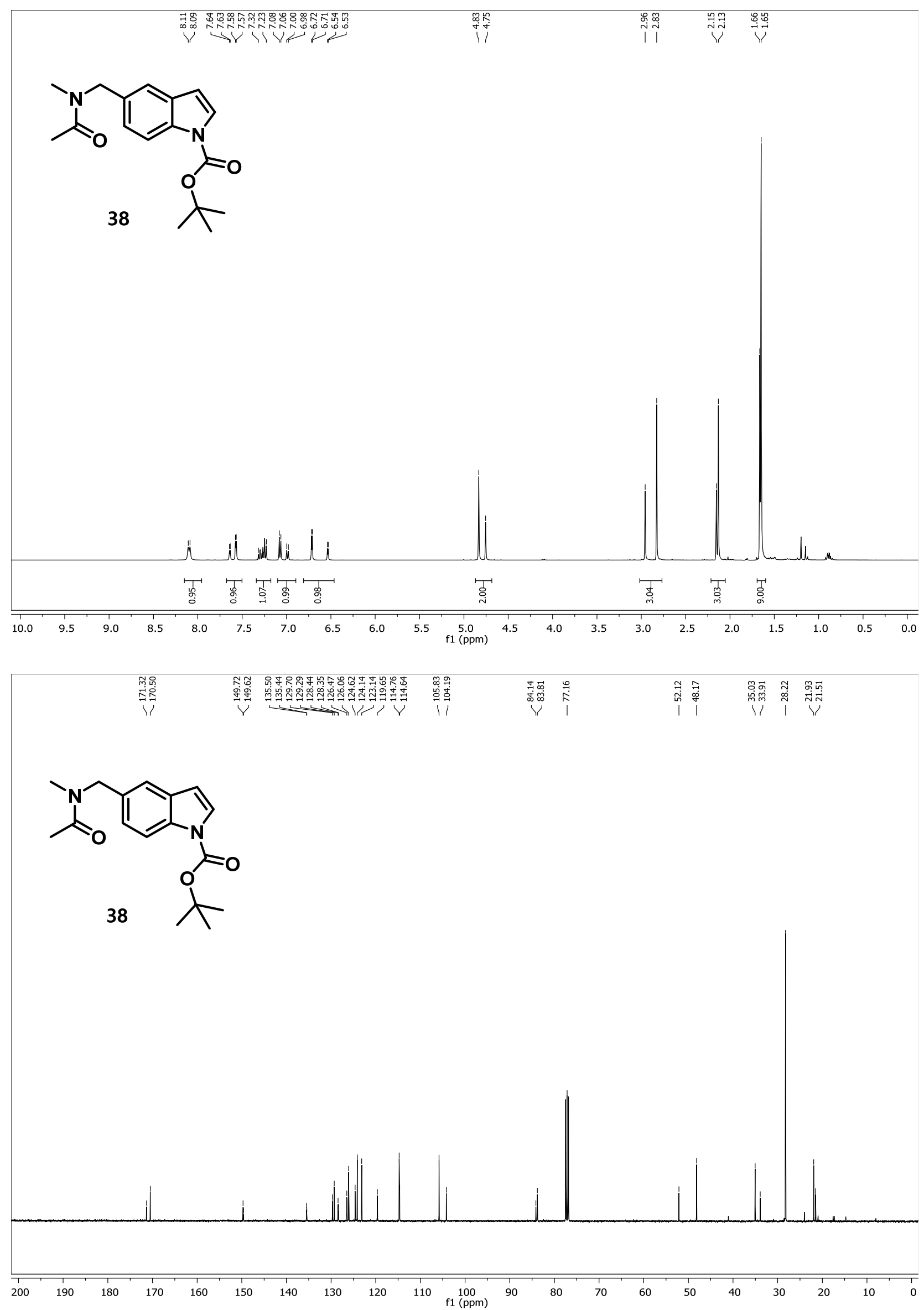

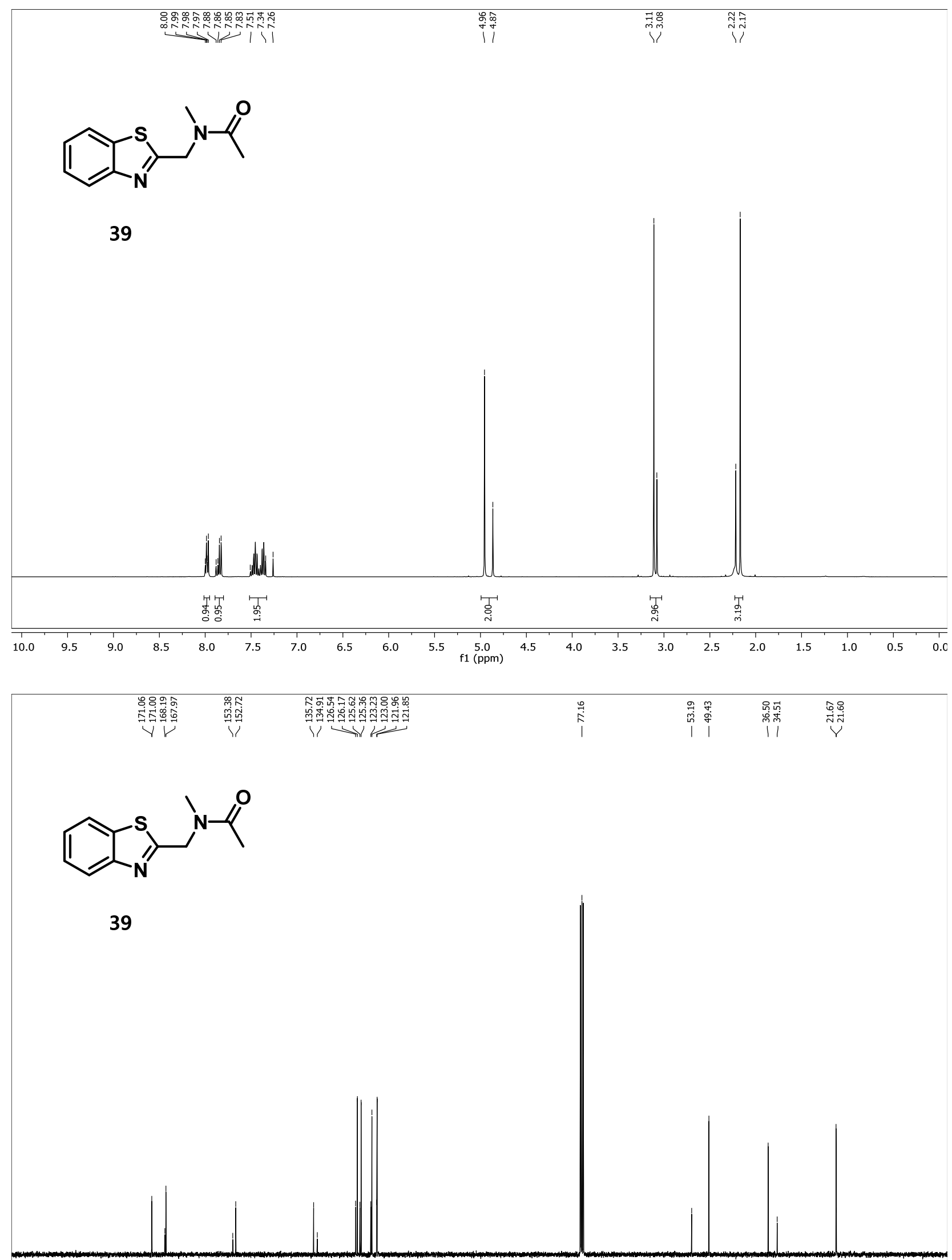

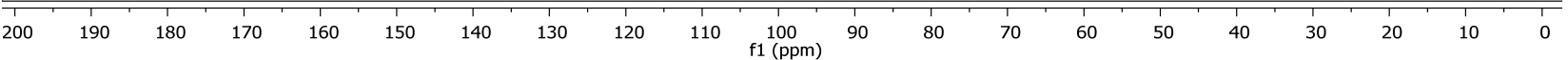



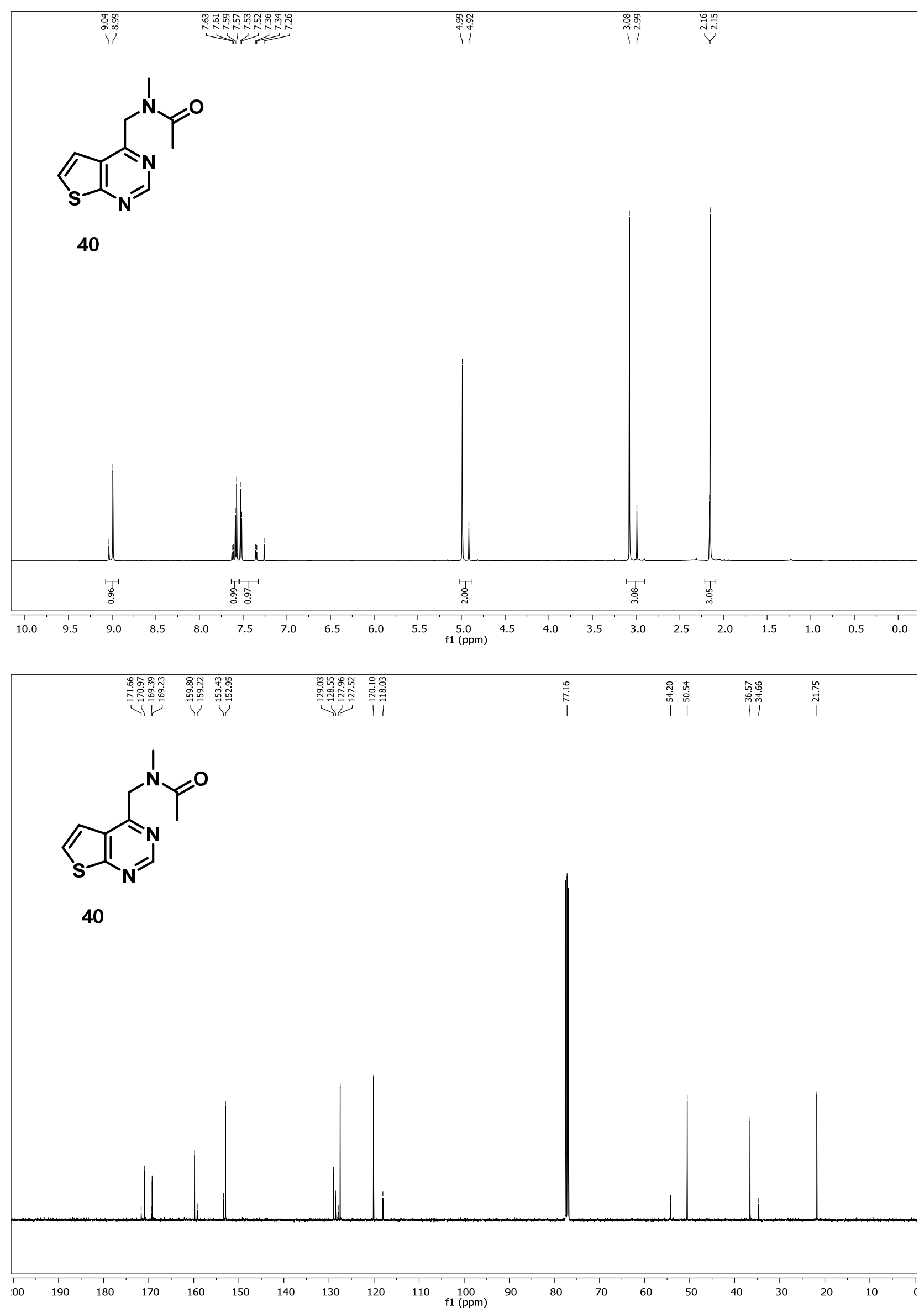

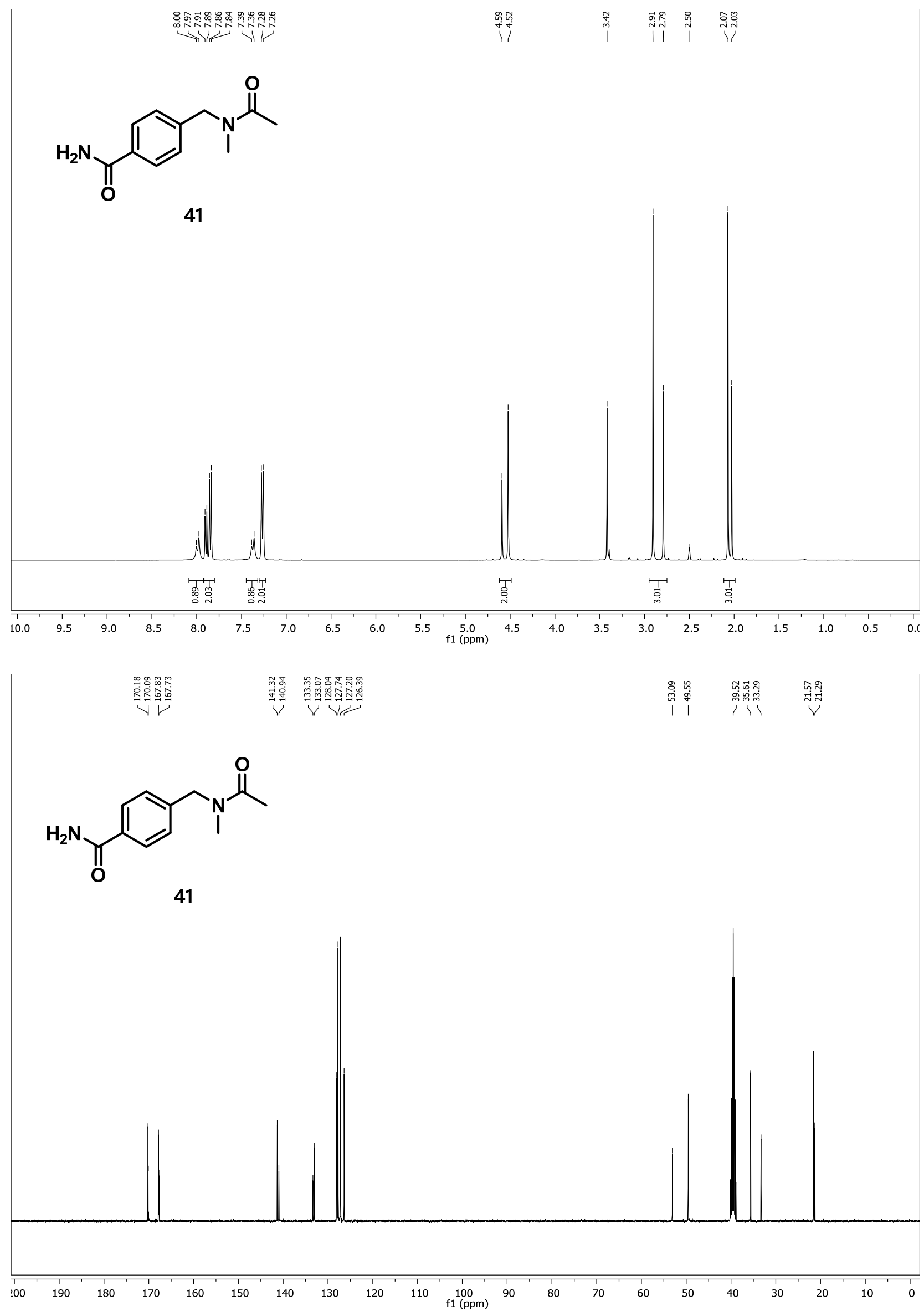

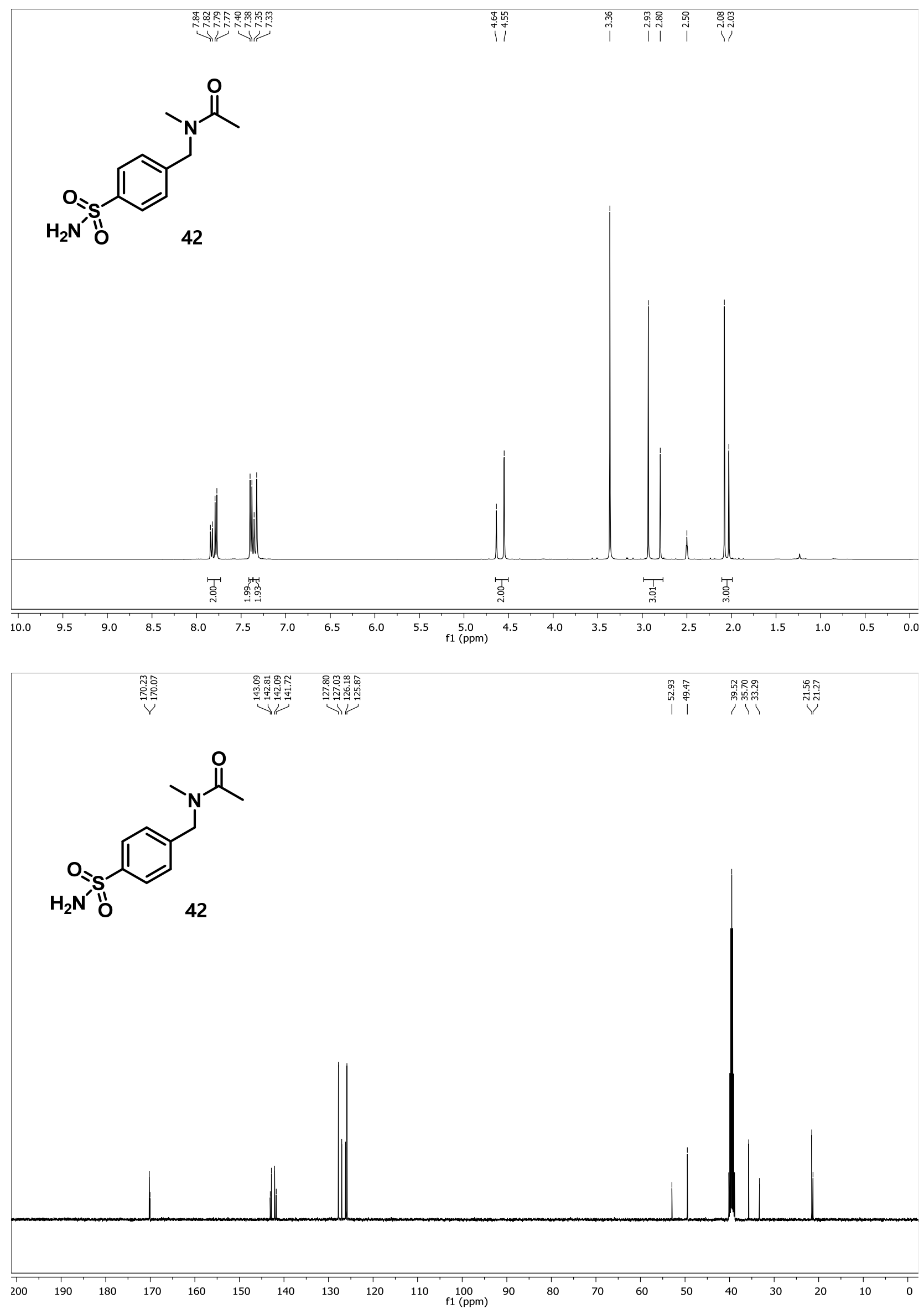

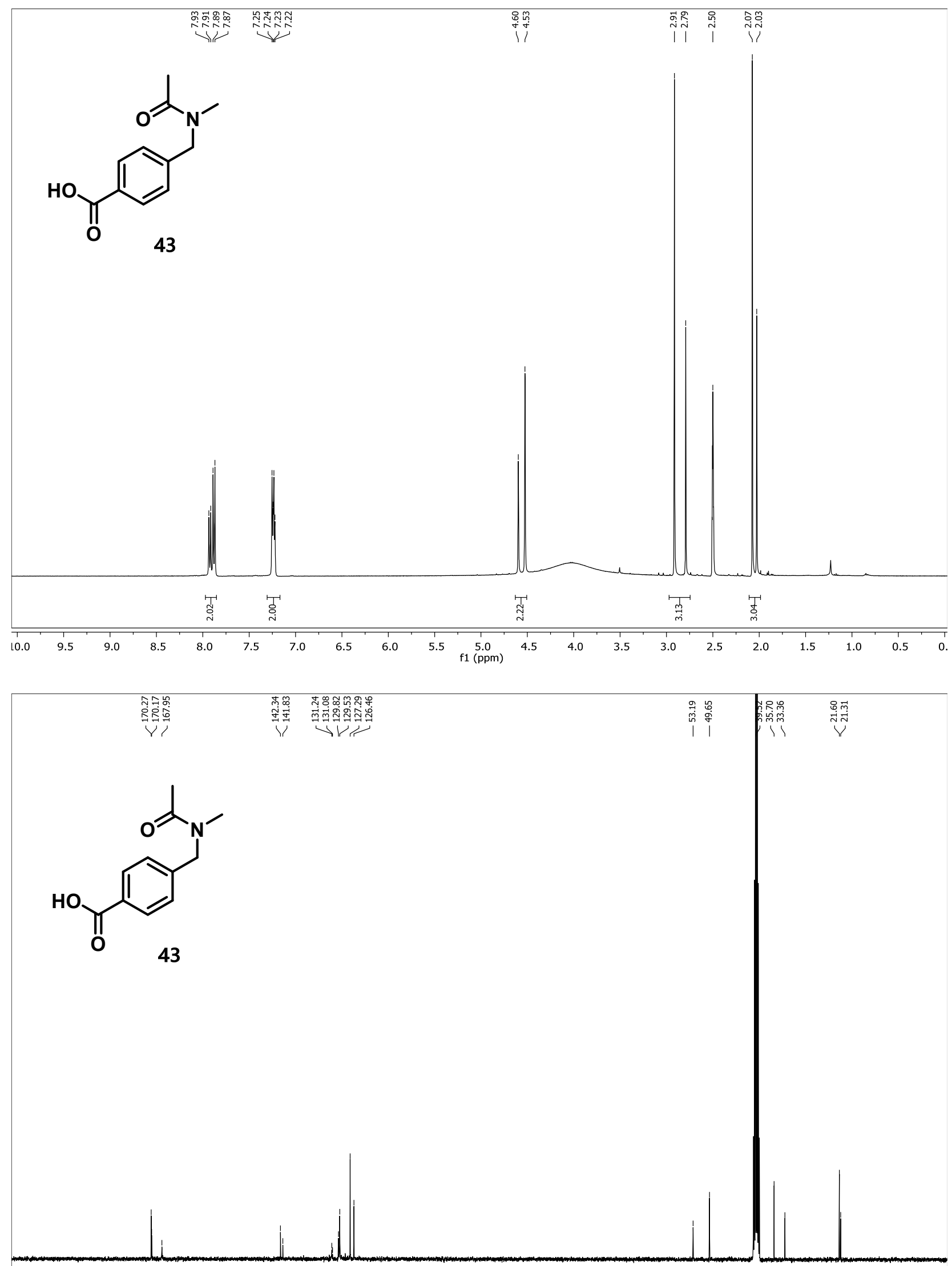

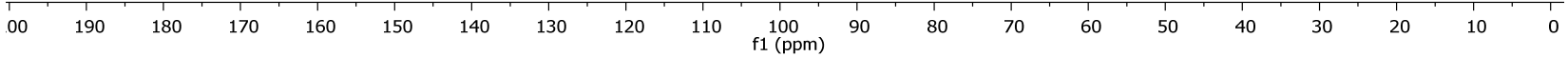



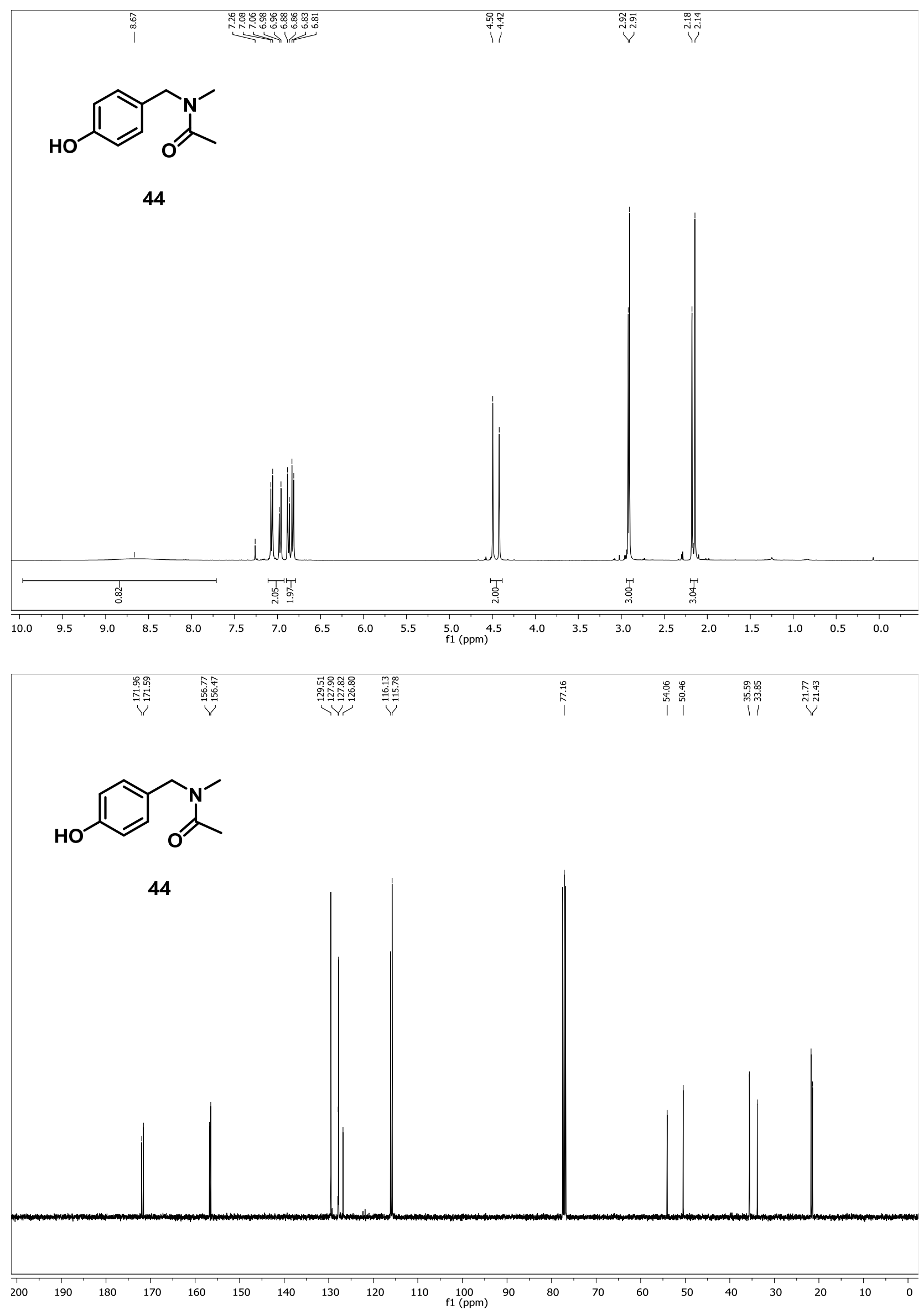

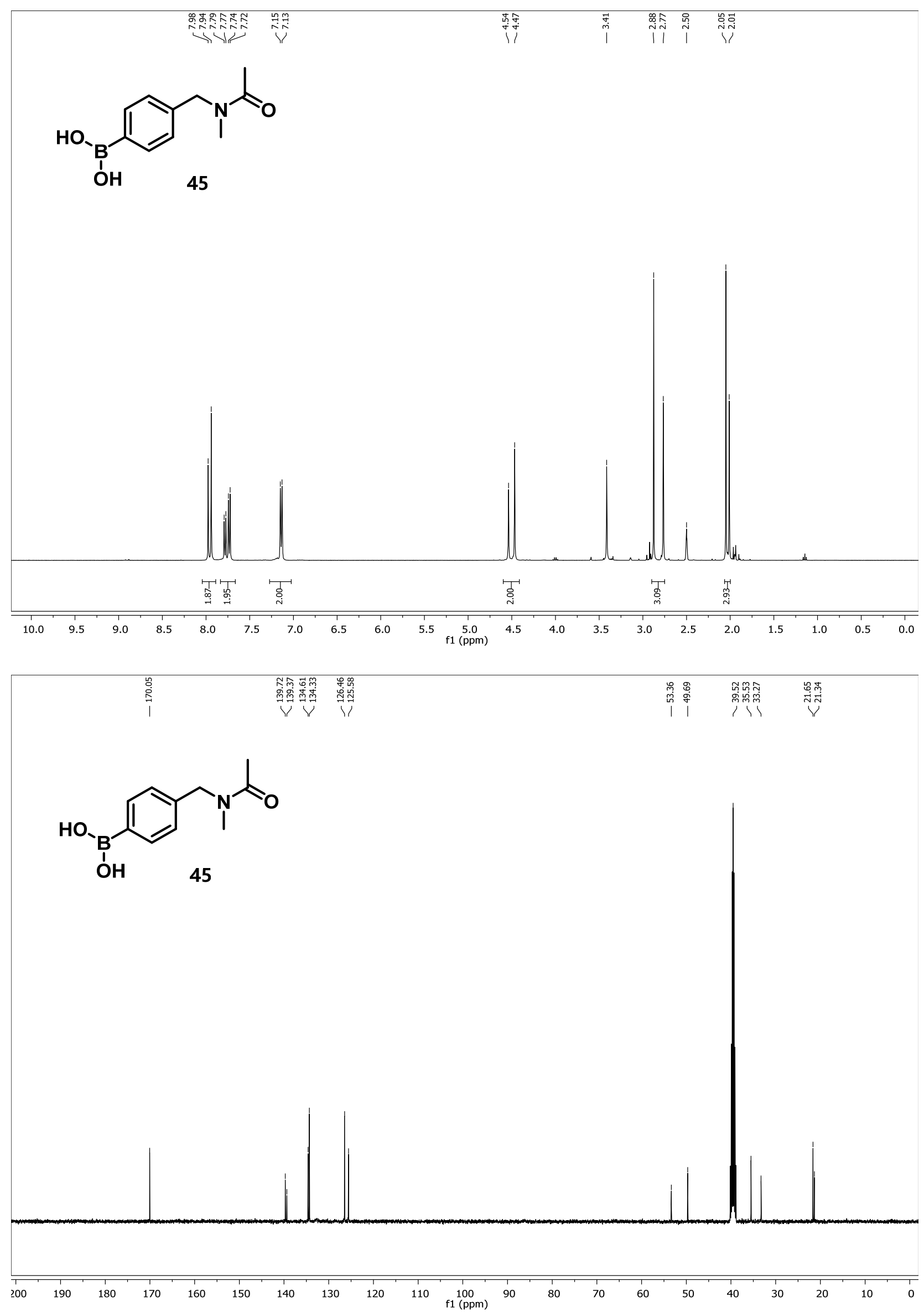

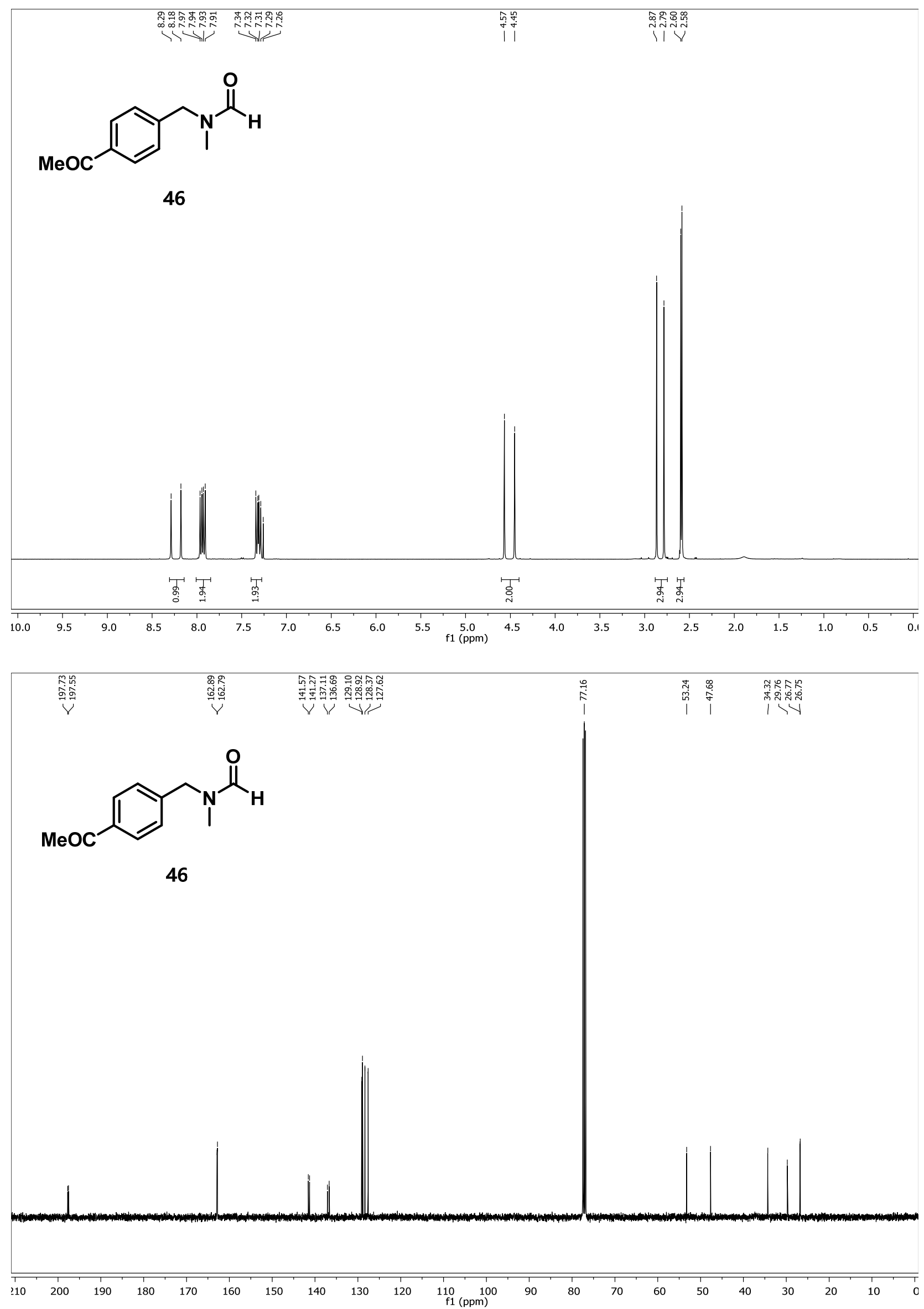

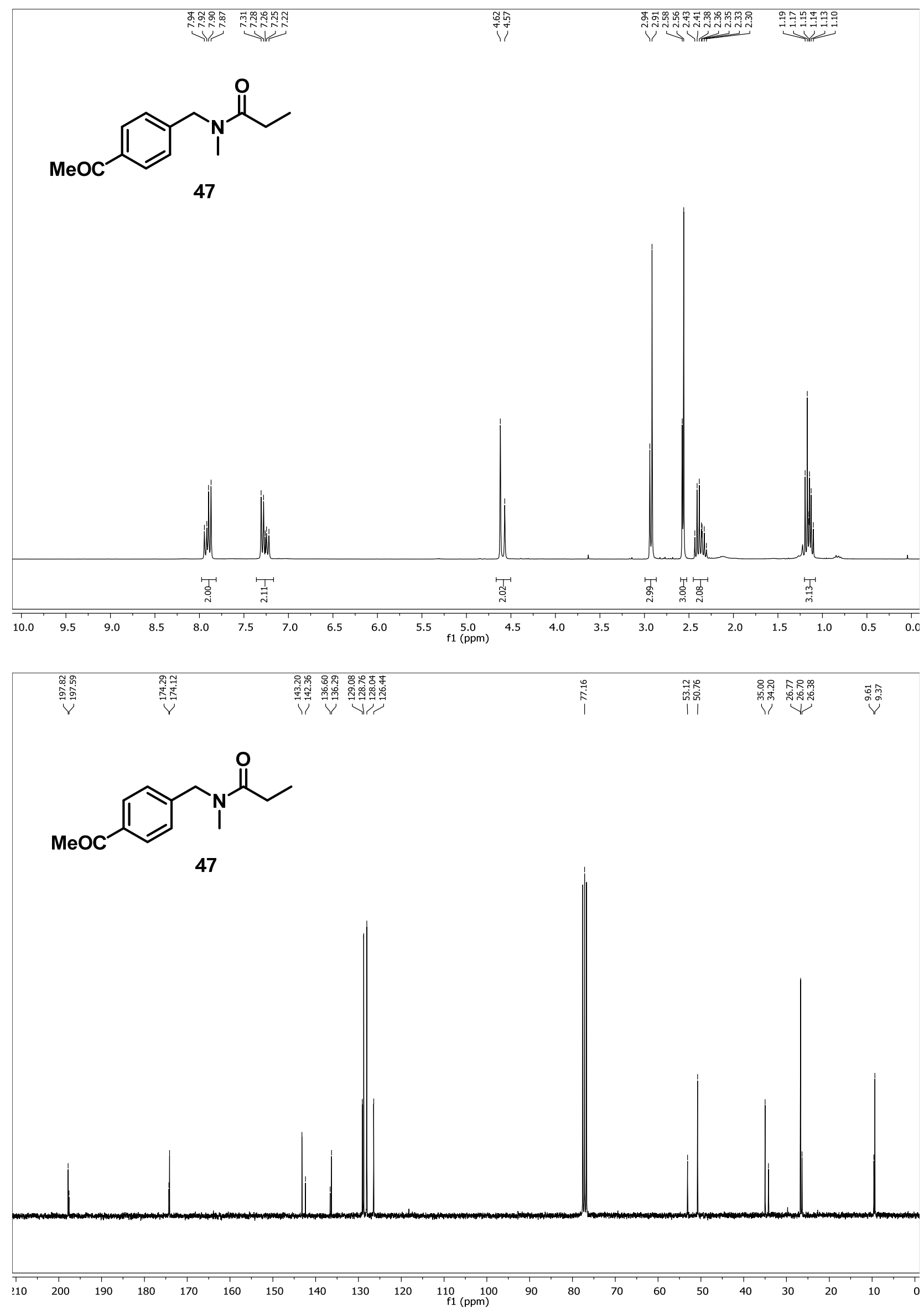

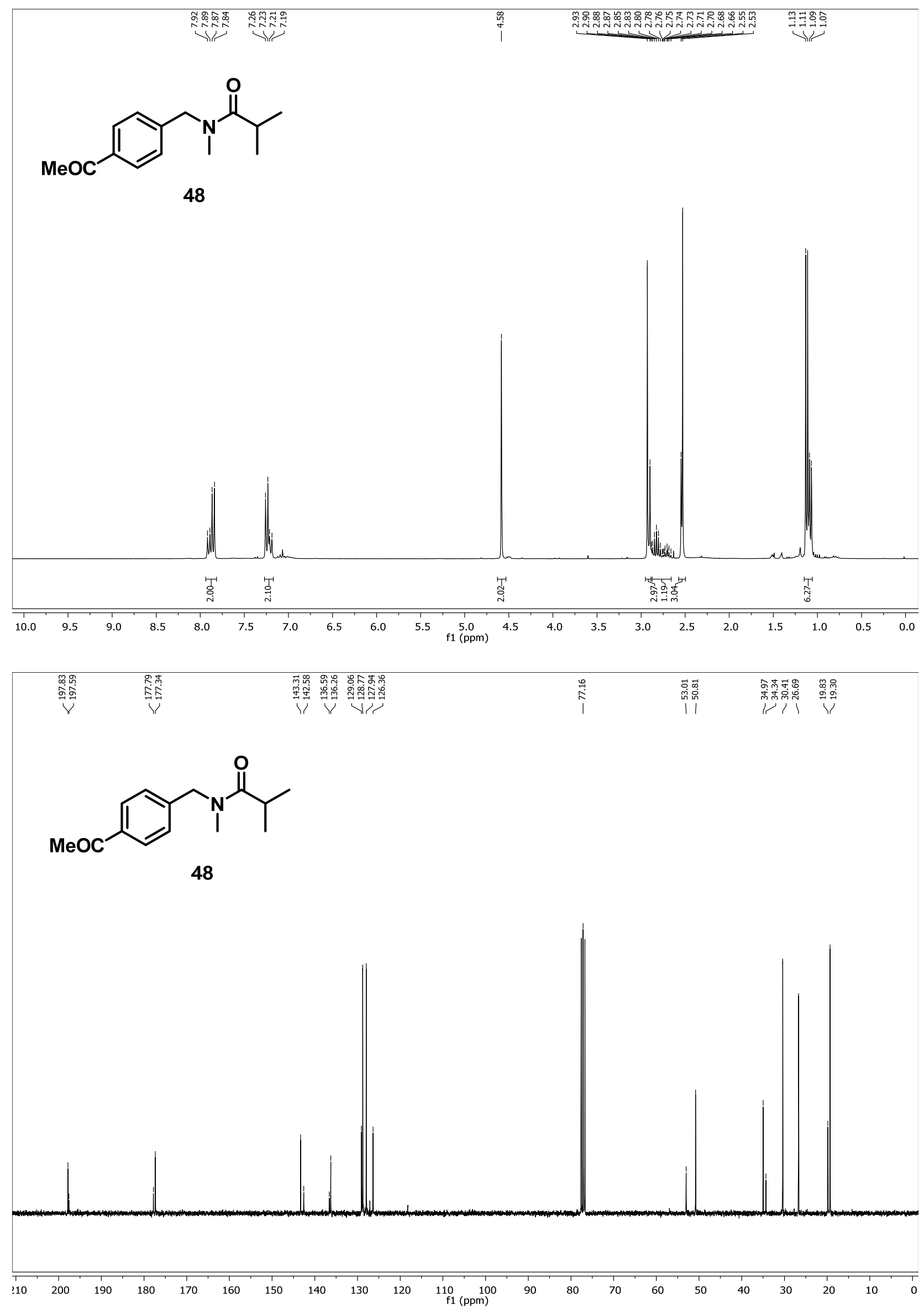

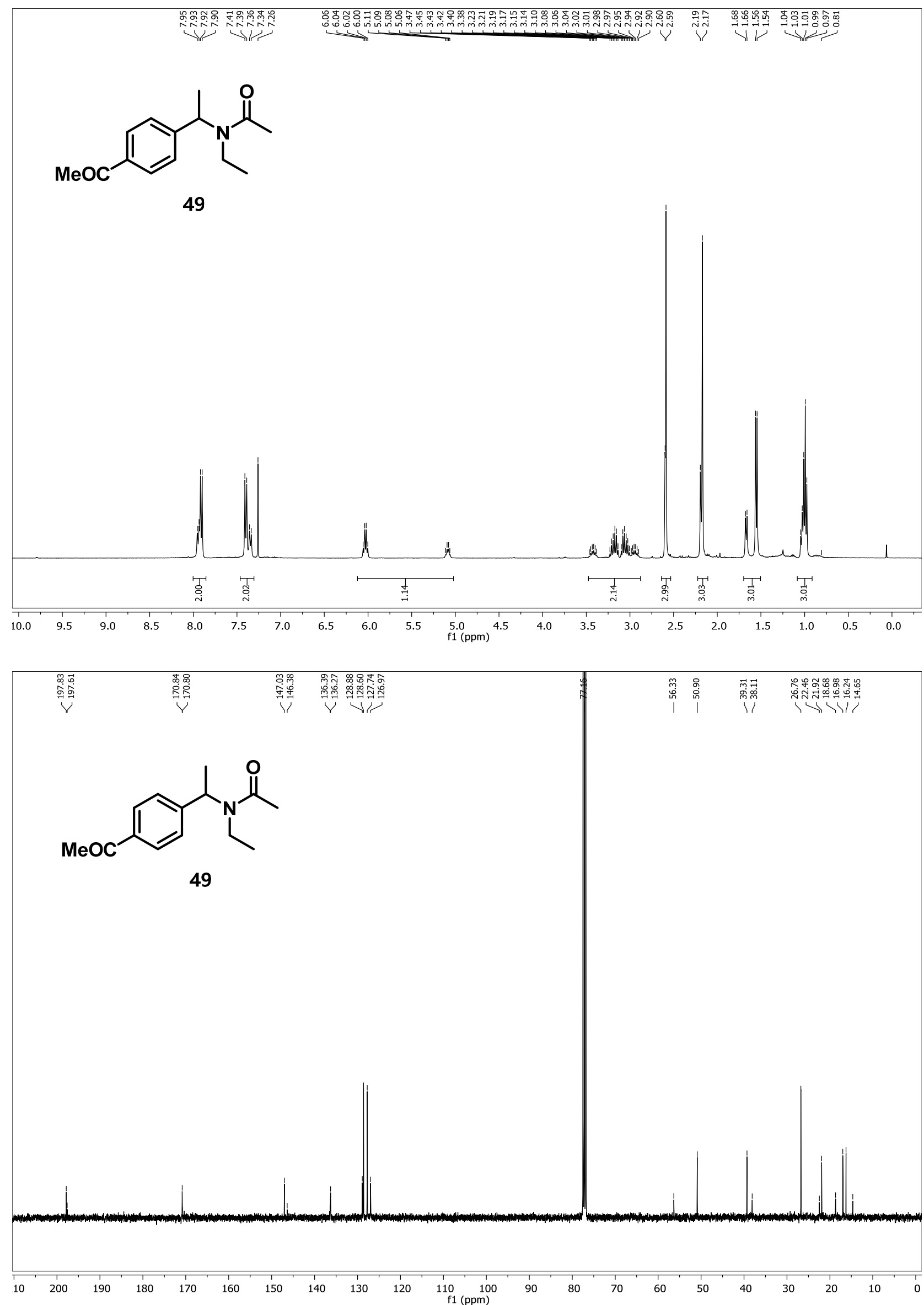

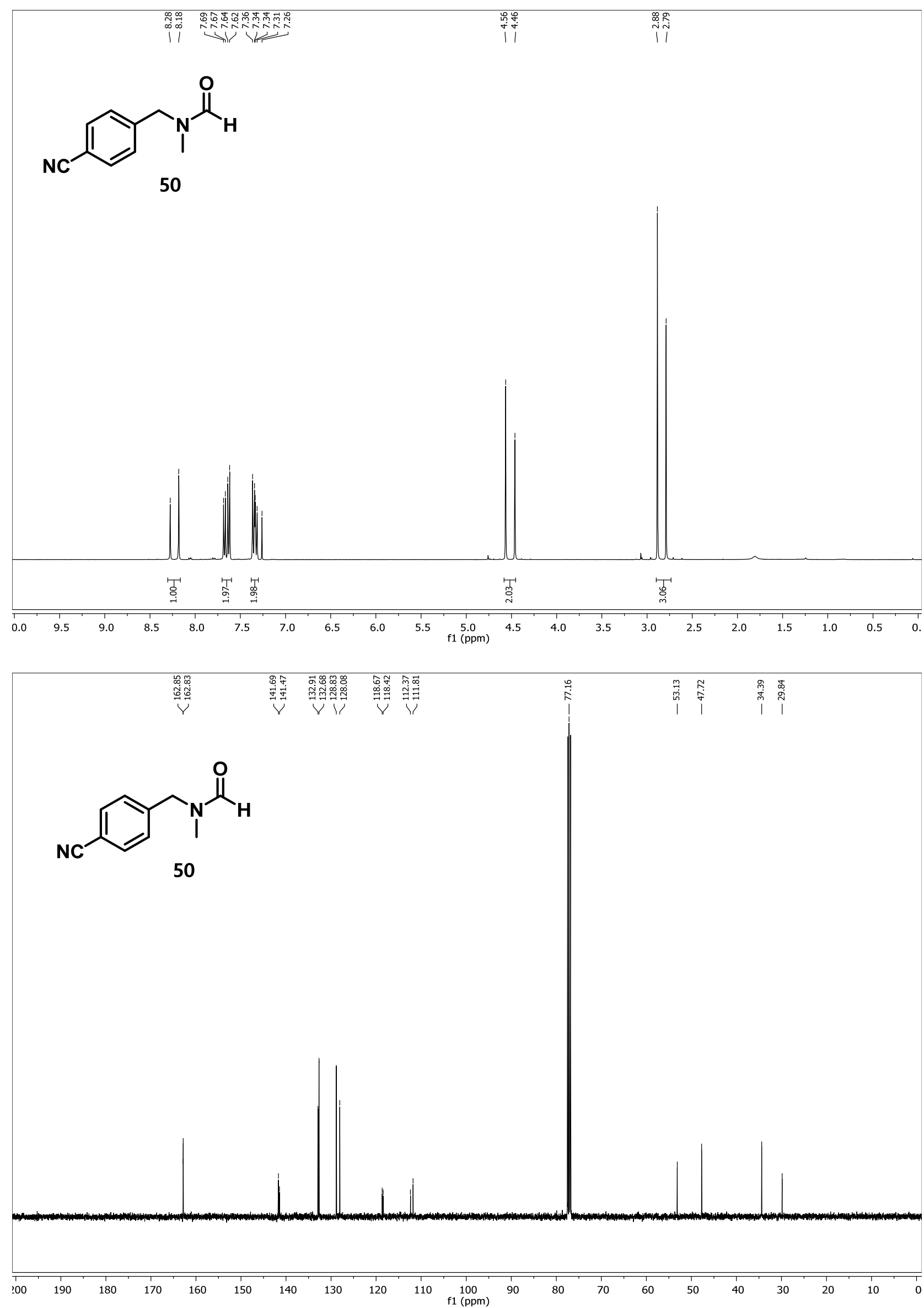

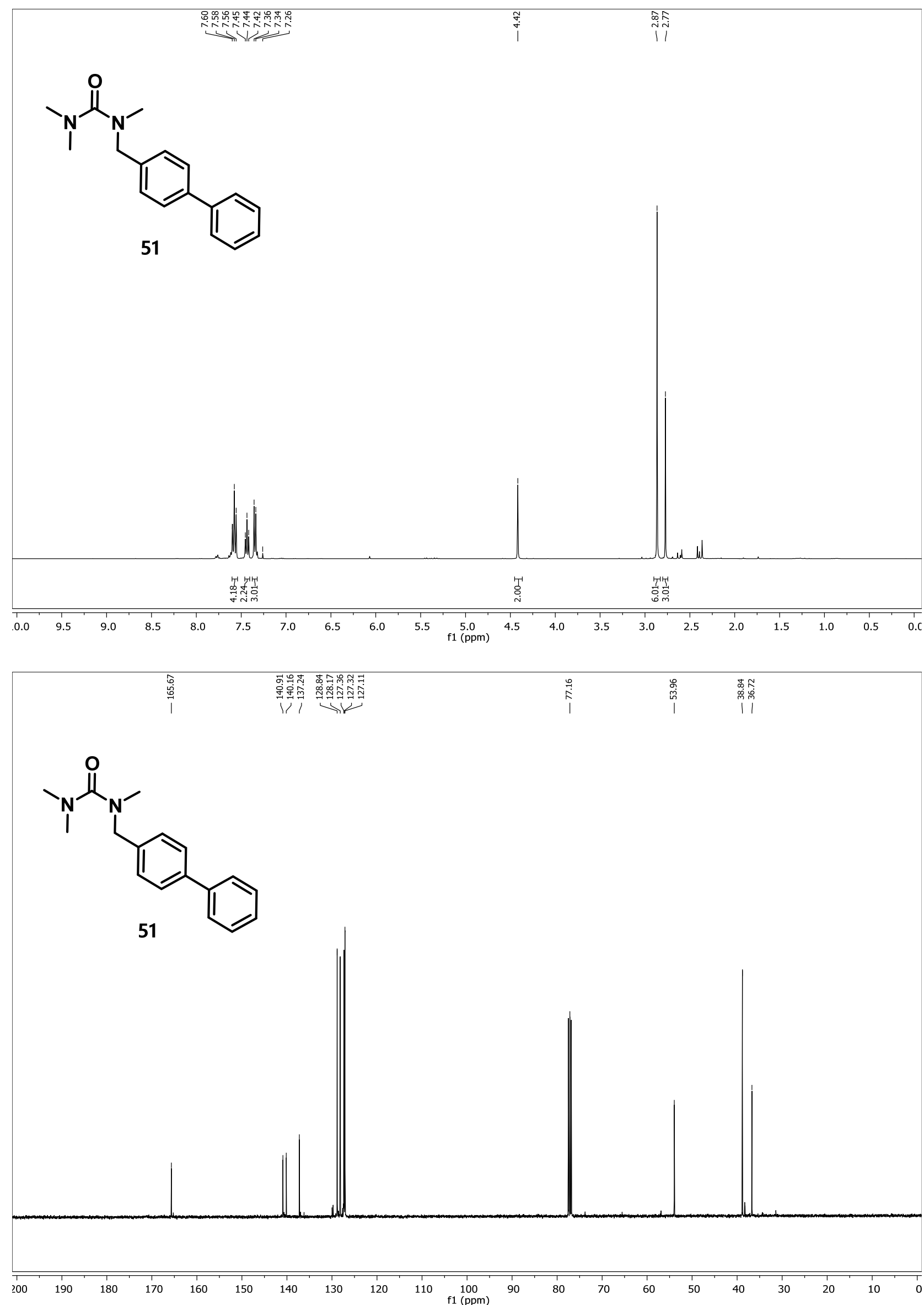

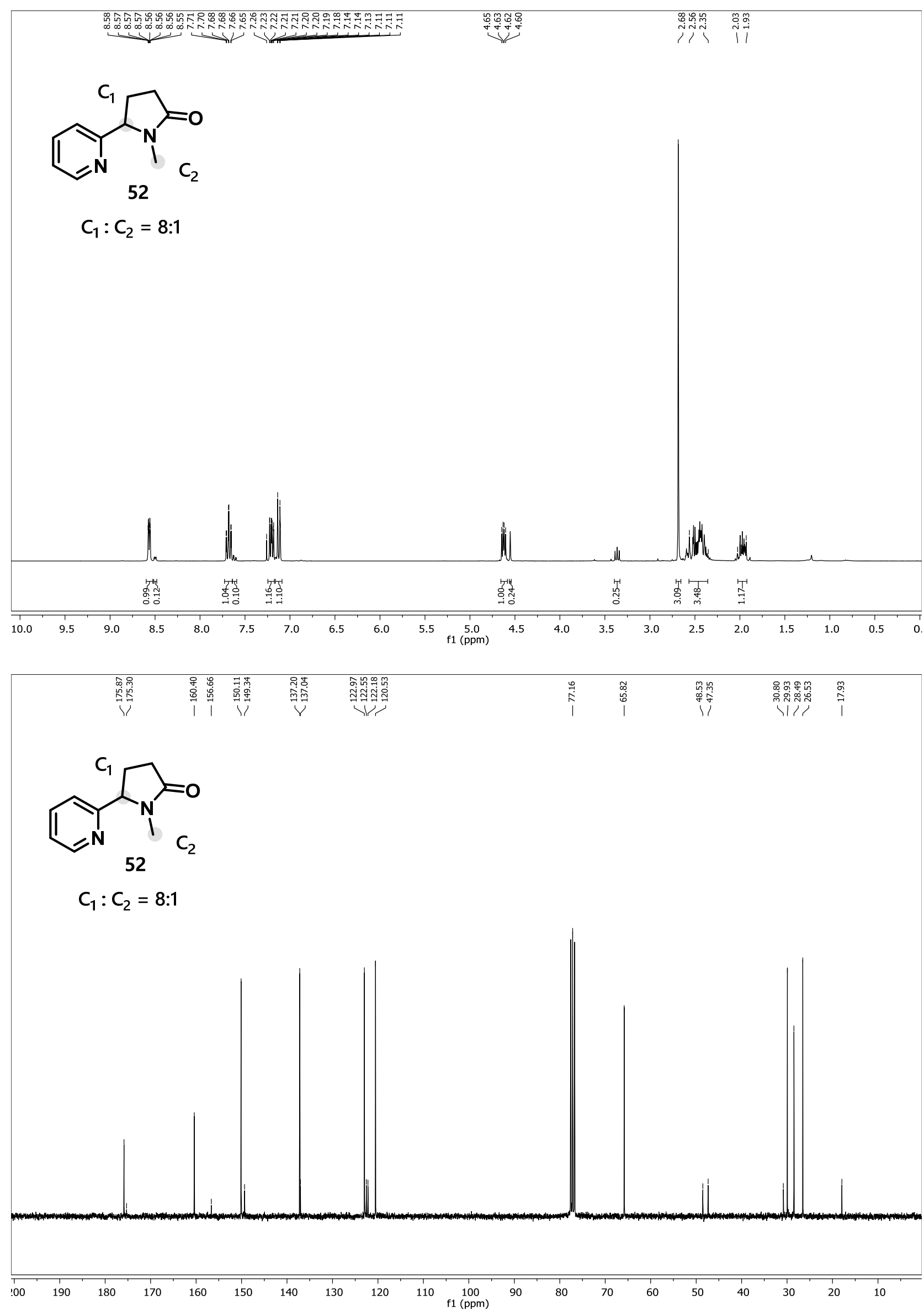

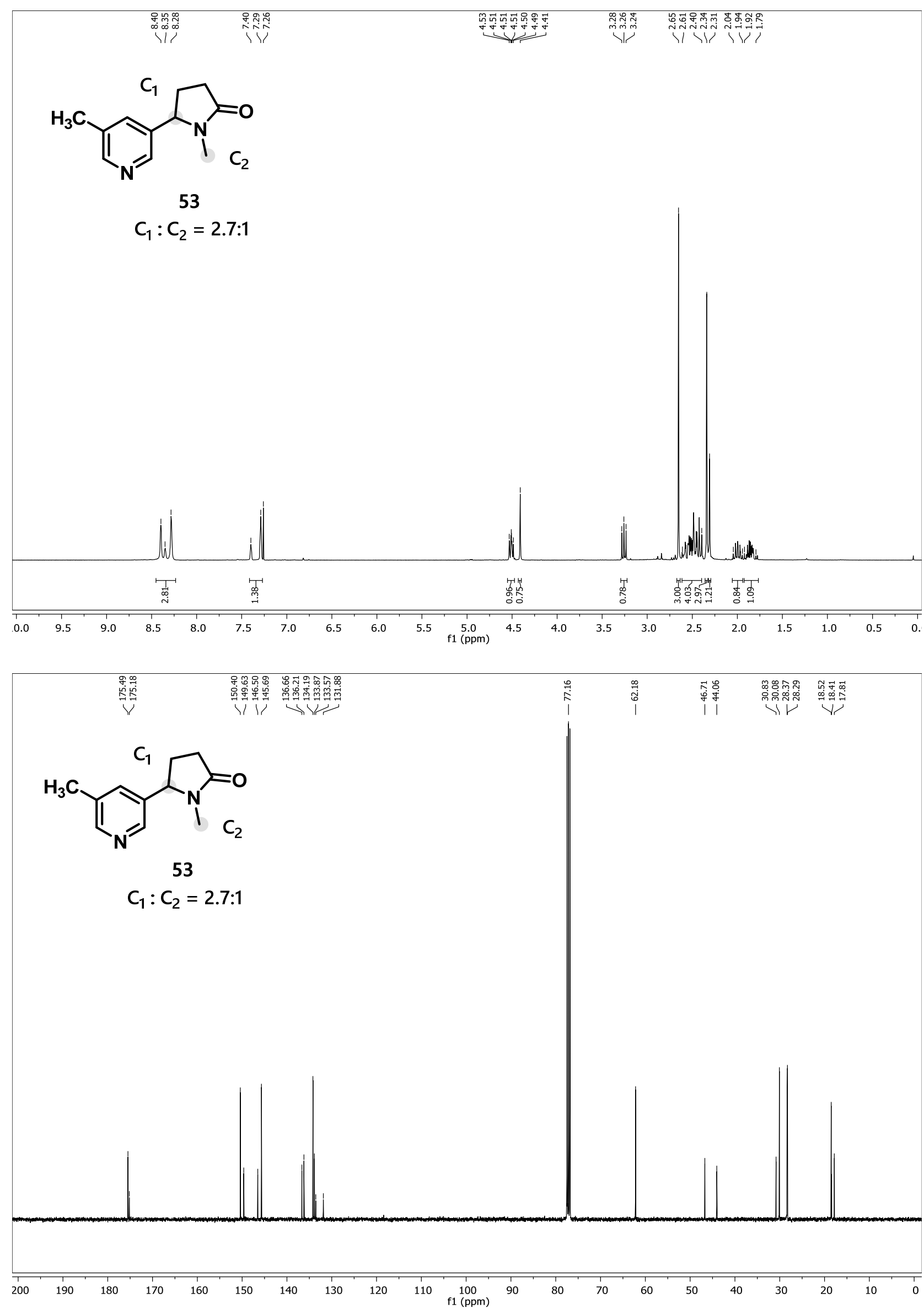

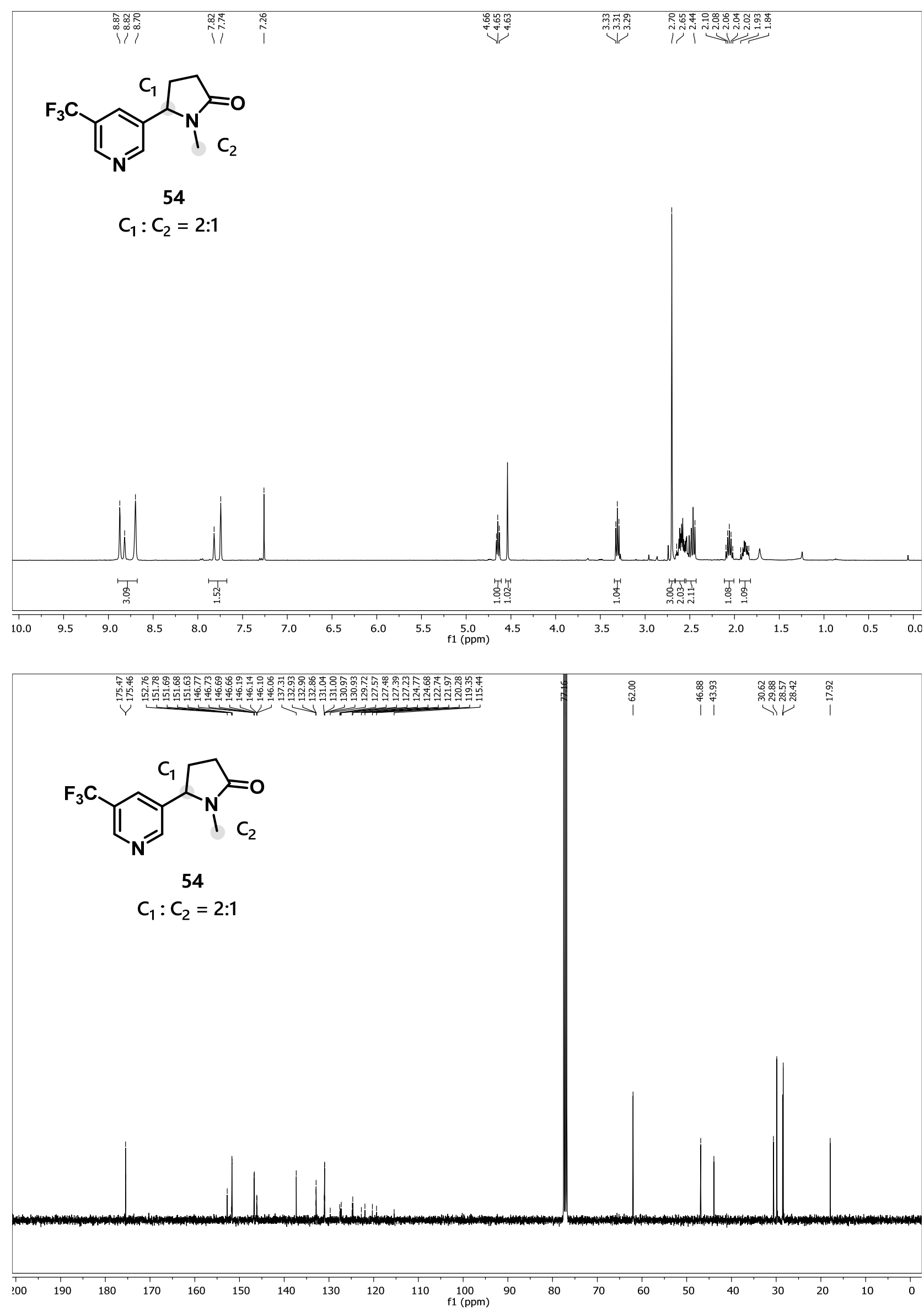

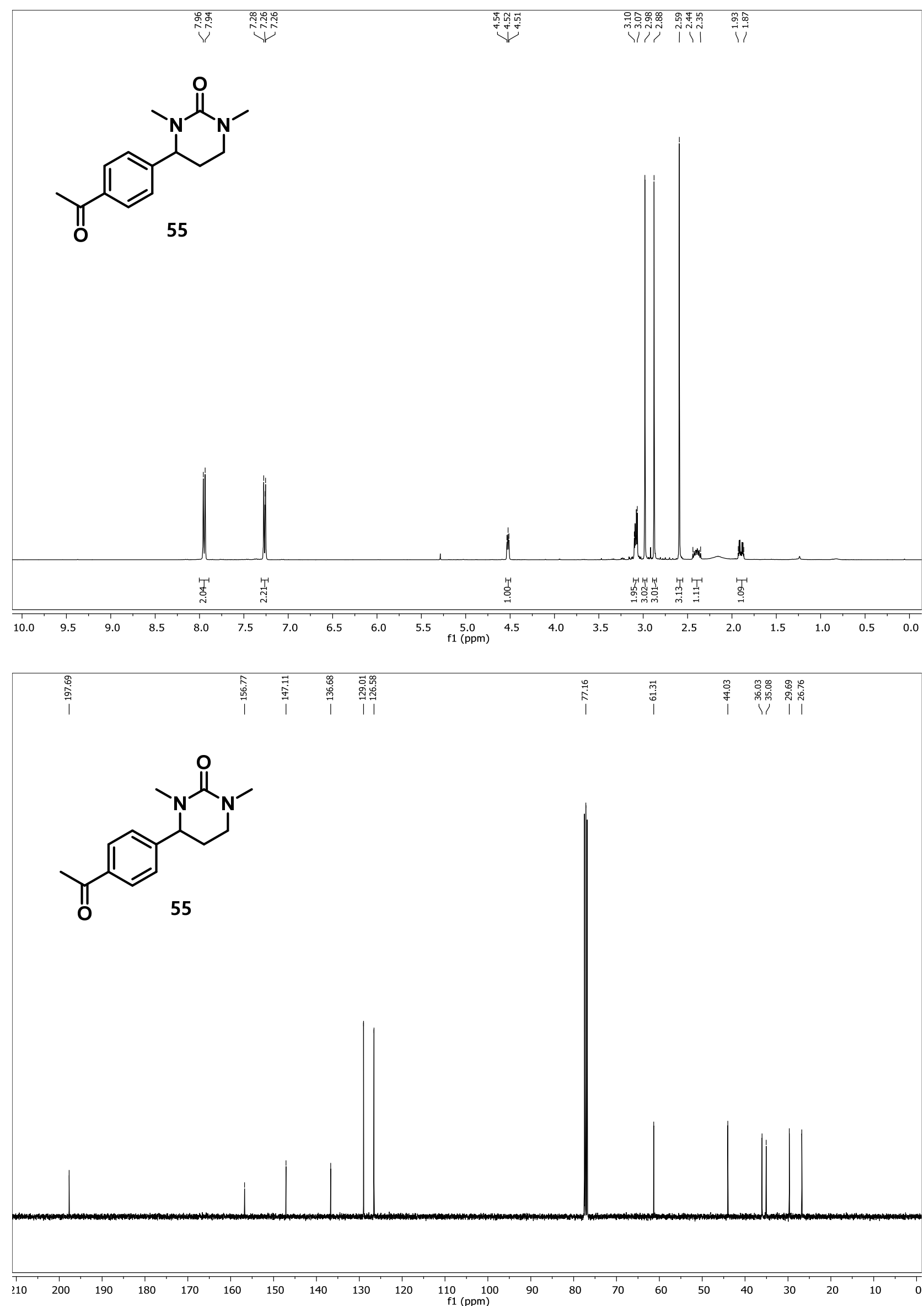

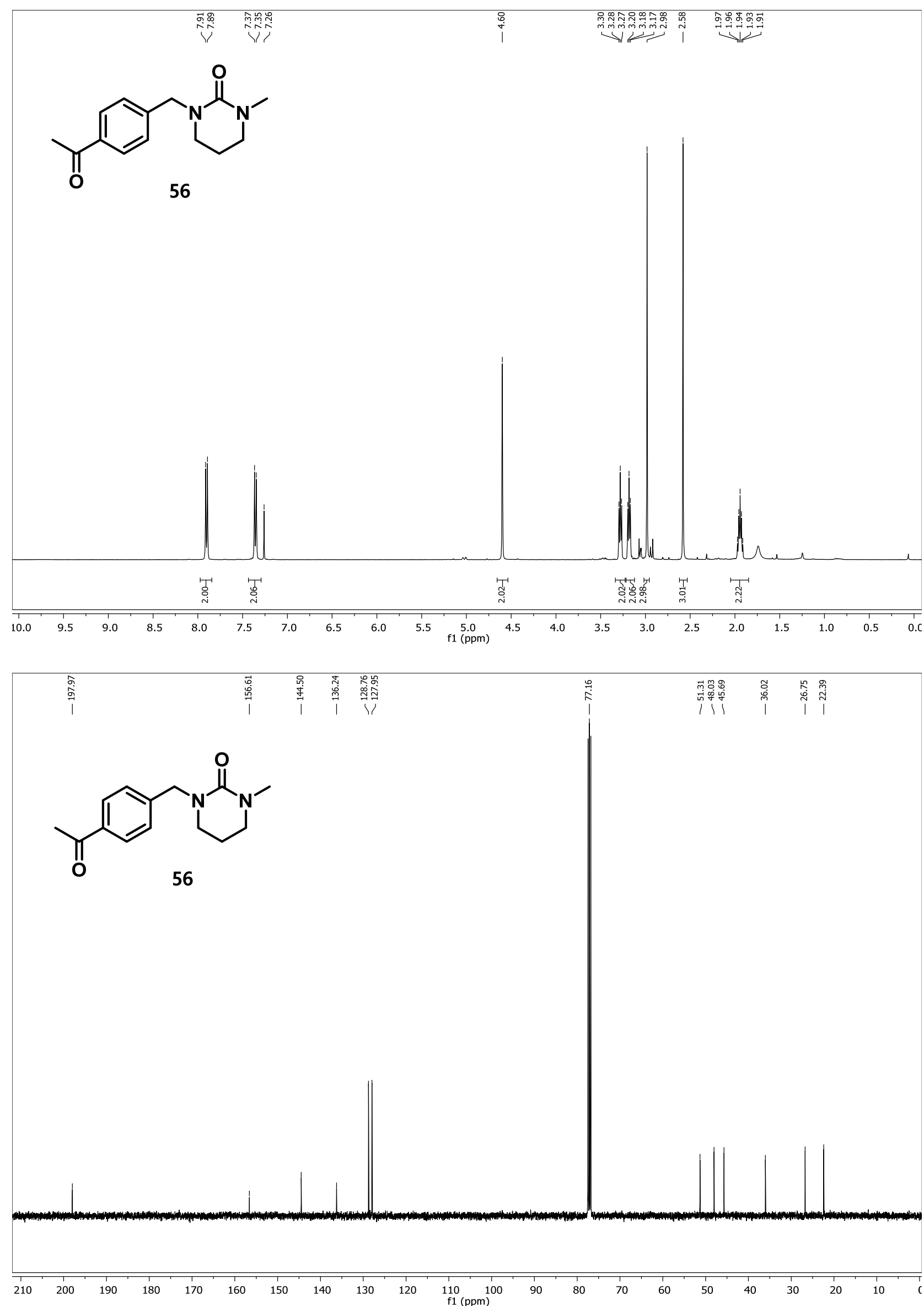

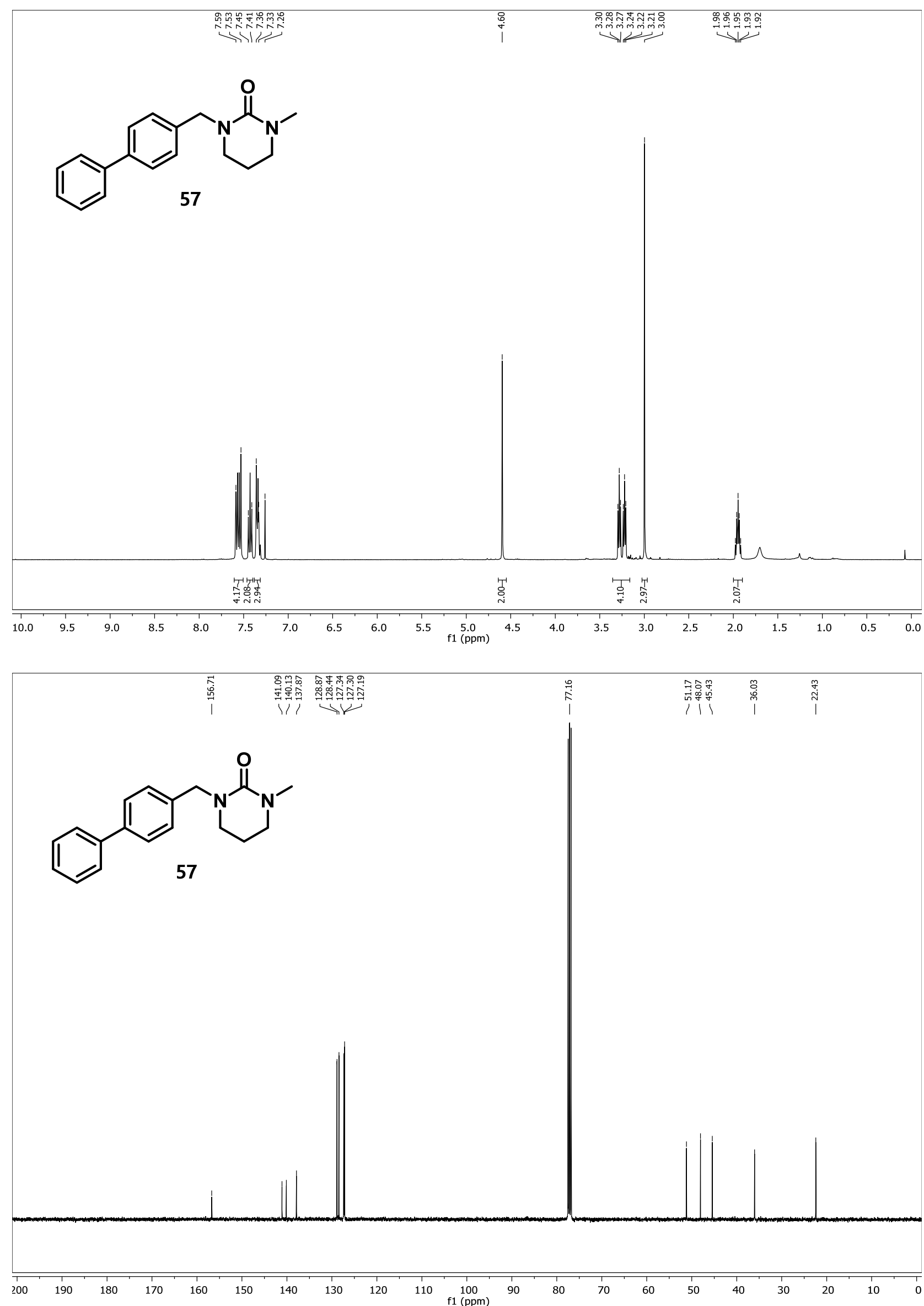

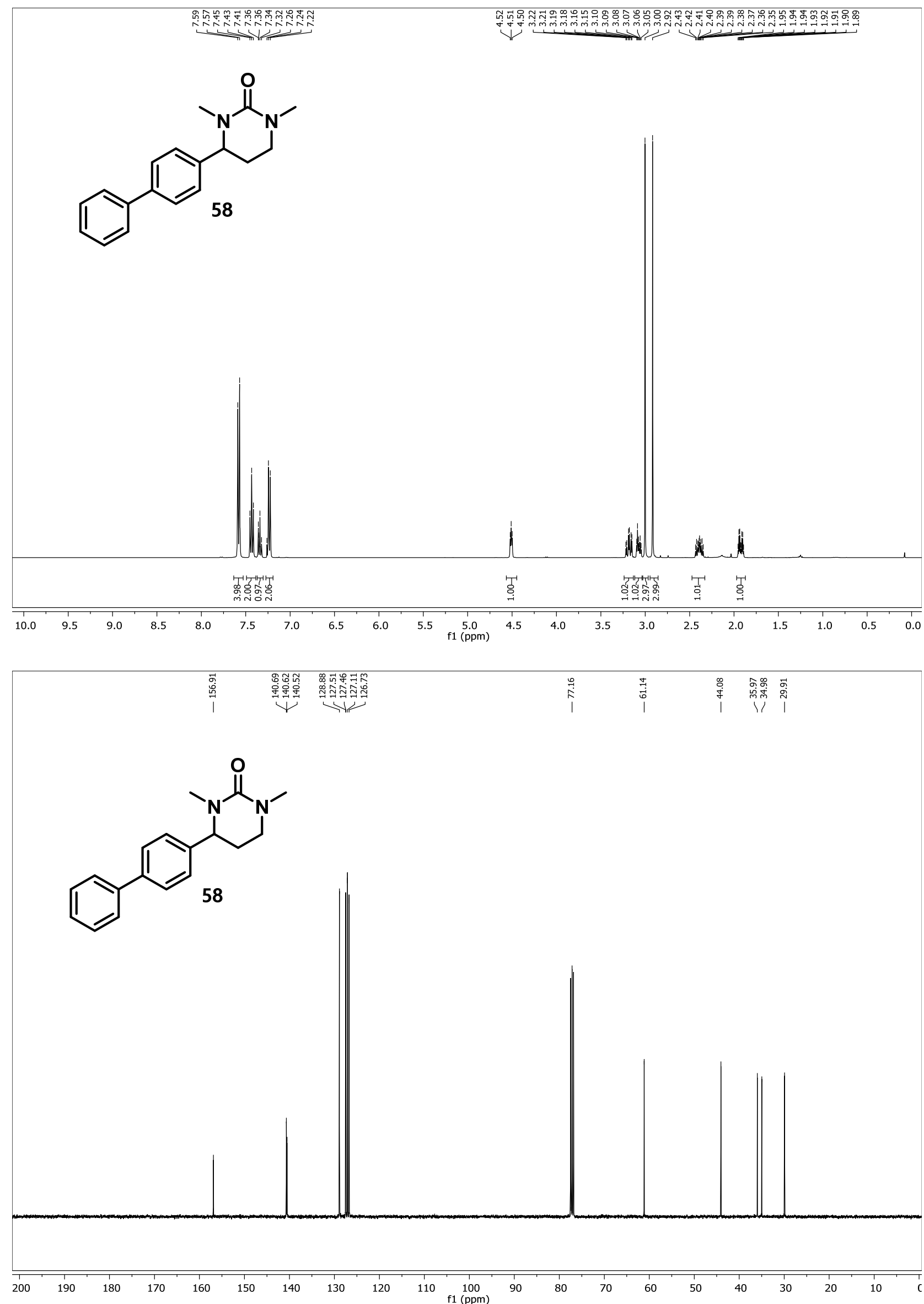


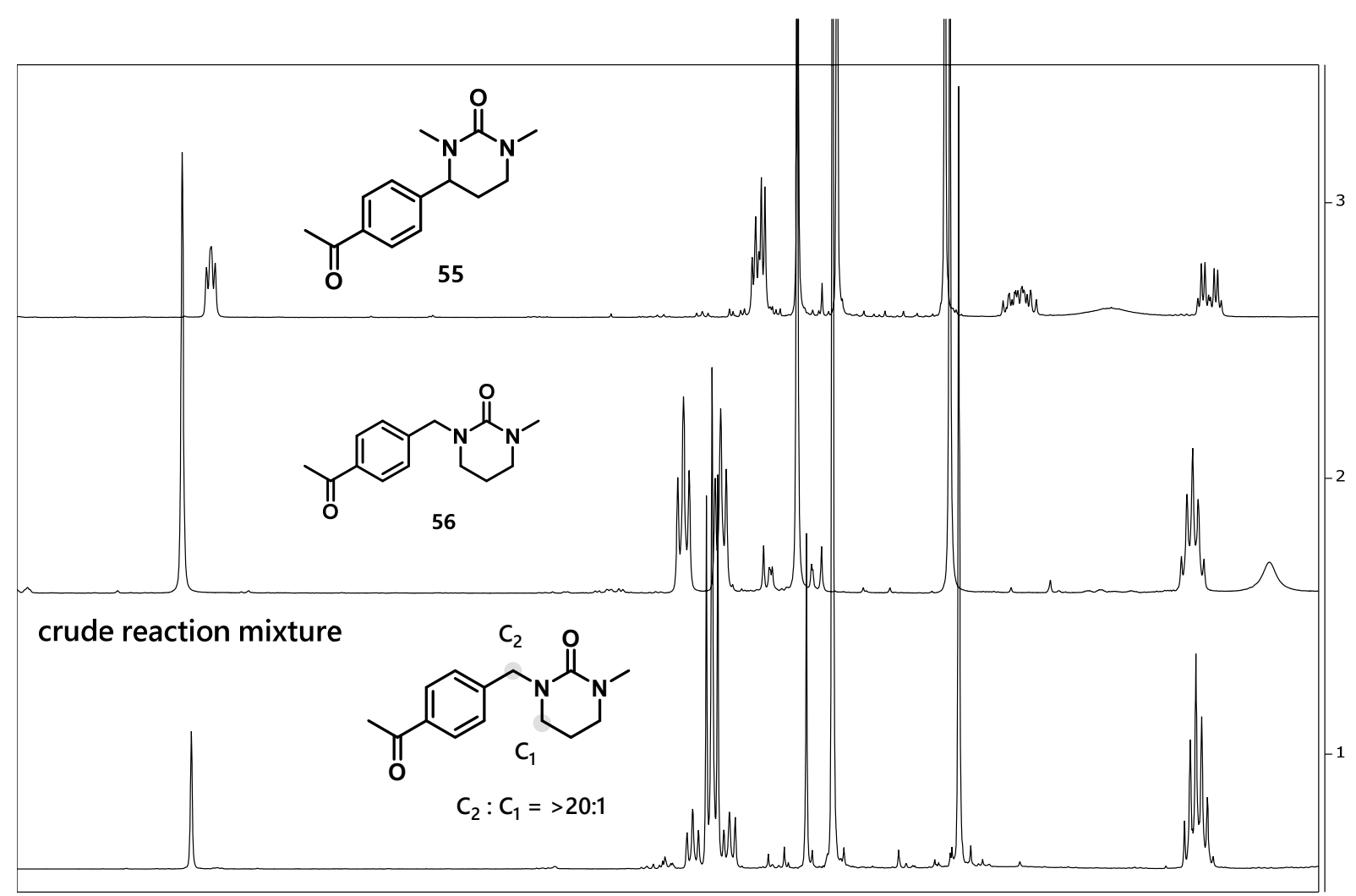

$\begin{array}{lllllllllllllllllllllllllllllllllllllllllll}5.0 & 4.9 & 4.8 & 4.7 & 4.6 & 4.5 & 4.4 & 4.3 & 4.2 & 4.1 & 4.0 & 3.9 & 3.8 & 3.7 & 3.6 & 3.5 & 3.4 & 3.3 & 3.2 & 3.1 & 3.0 & 2.9 & 2.8 & 2.7 & 2.6 & 2.5 & 2.4 & 2.3 & 2.2 & 2.1 & 2.0 & 1.9 & 1.8 & 1.7\end{array}$

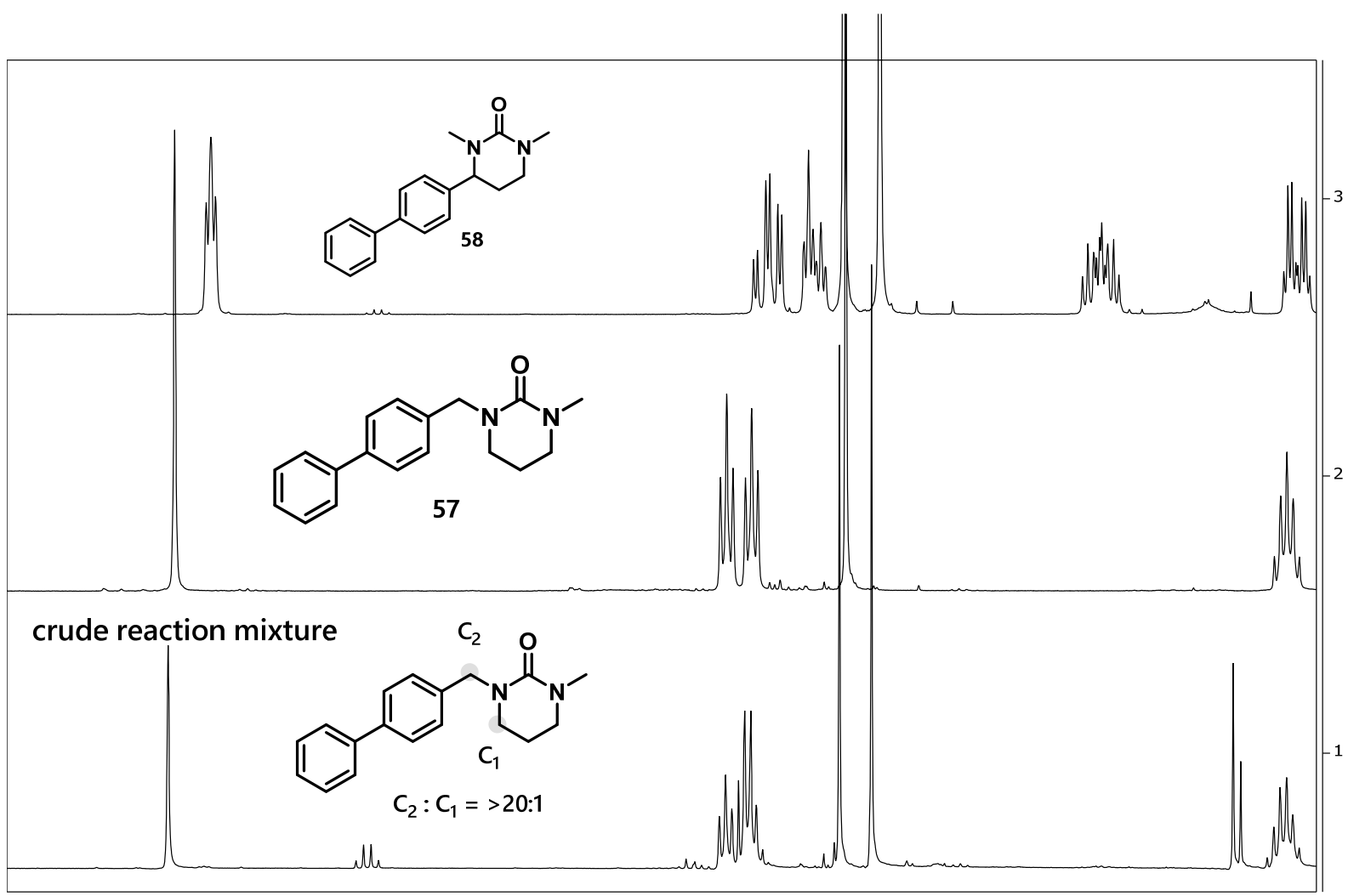

$\begin{array}{llllllllllllllllllllllllllllllllllllllllllllll}4.9 & 4.8 & 4.7 & 4.6 & 4.5 & 4.4 & 4.3 & 4.2 & 4.1 & 4.0 & 3.9 & 3.8 & 3.7 & 3.6 & 3.5 & 3.4 & 3.3 & 3.2 & 3.1 & 3.0 & 2.9 & 2.8 & 2.7 & 2.6 & 2.5 & 2.4 & 2.3 & 2.2 & 2.1 & 2.0 & 1.9\end{array}$ 

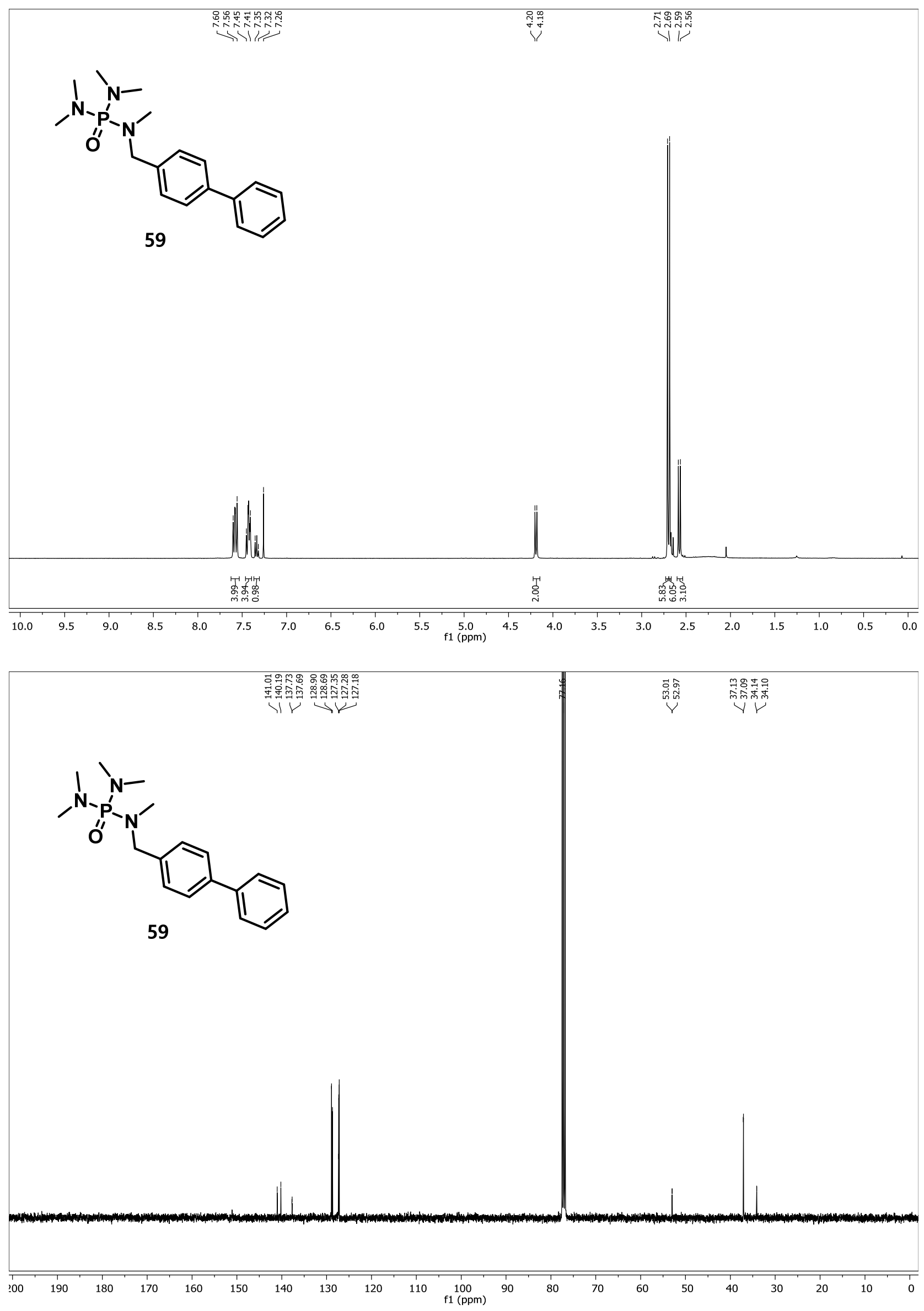

S140 

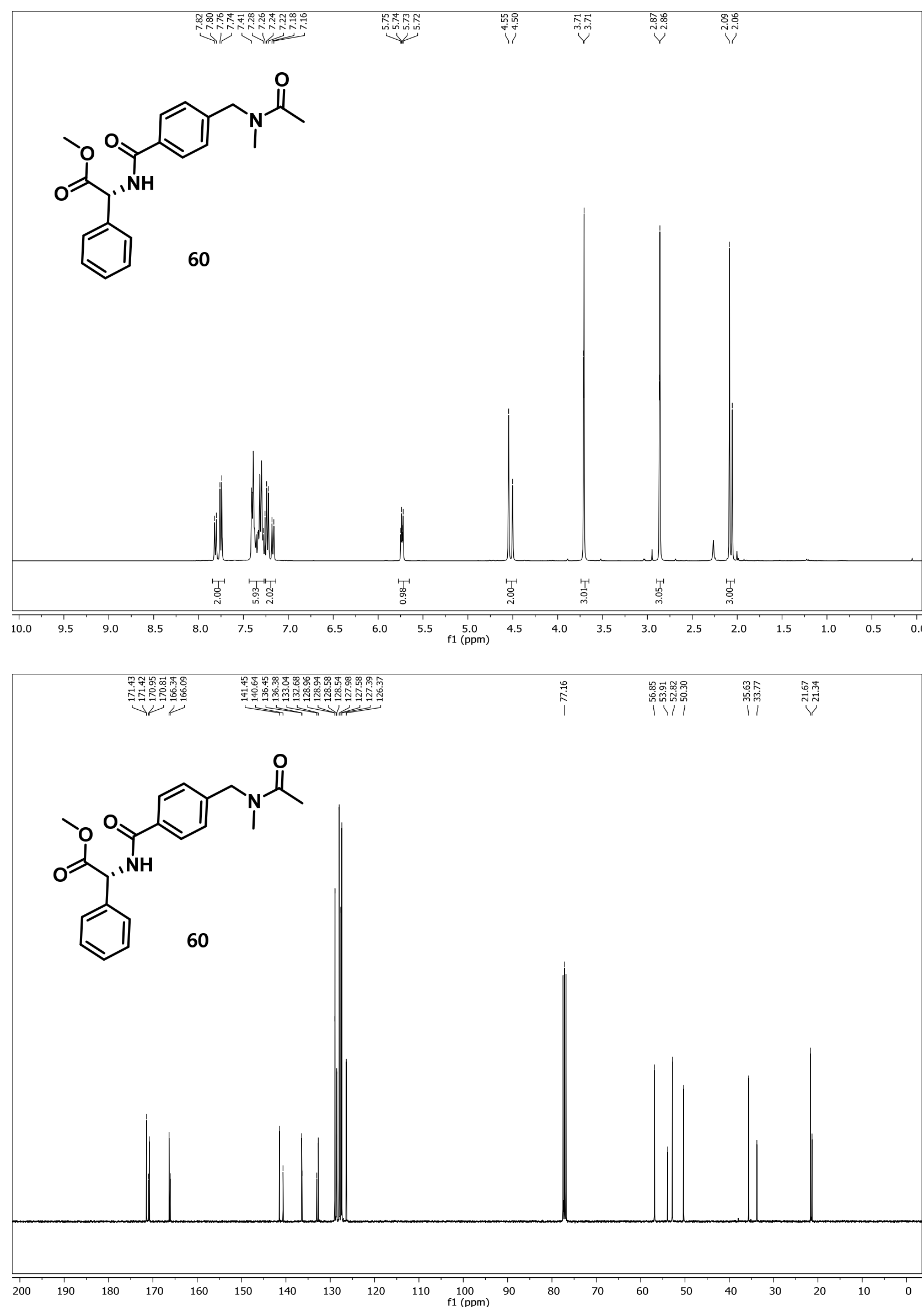

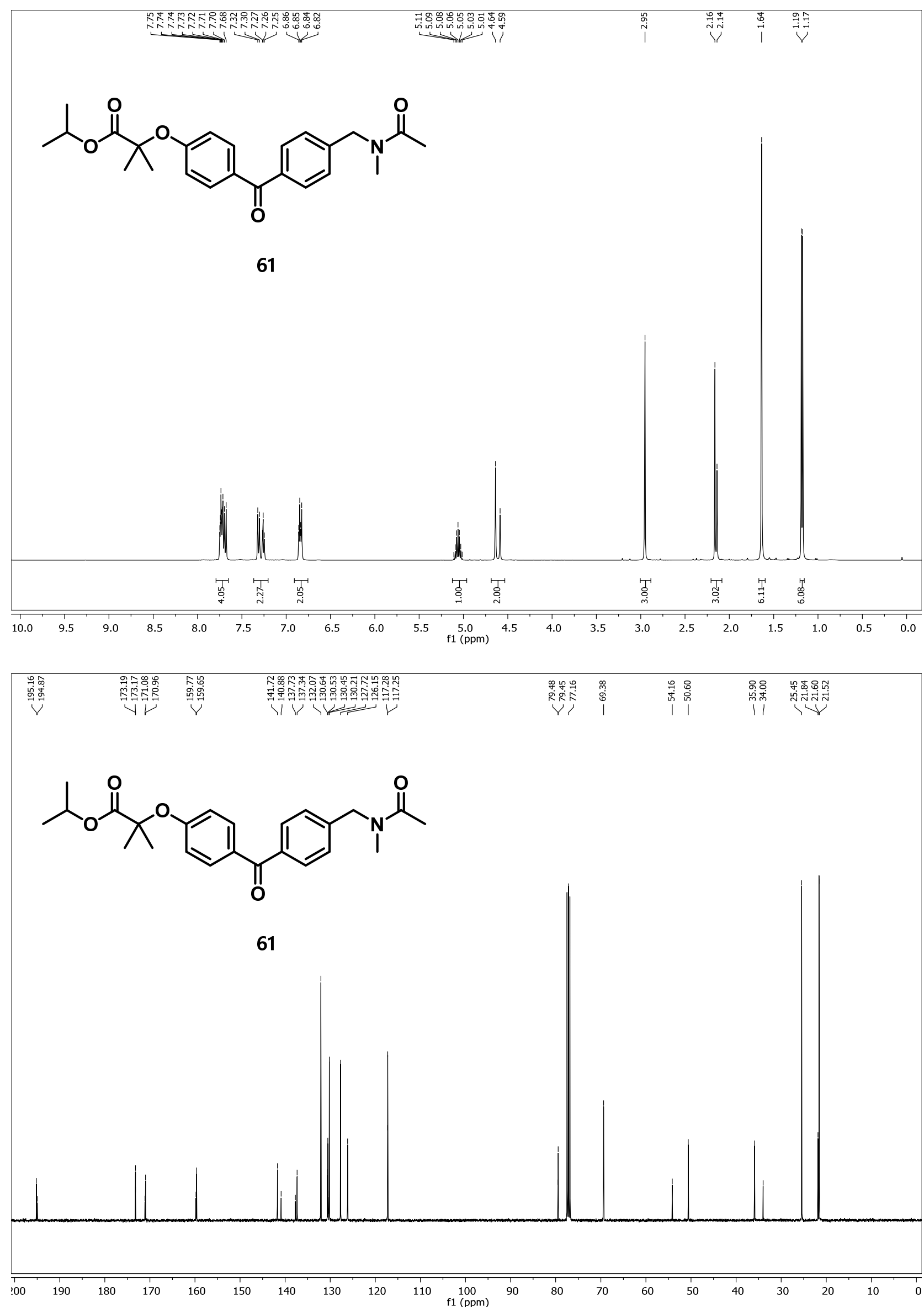

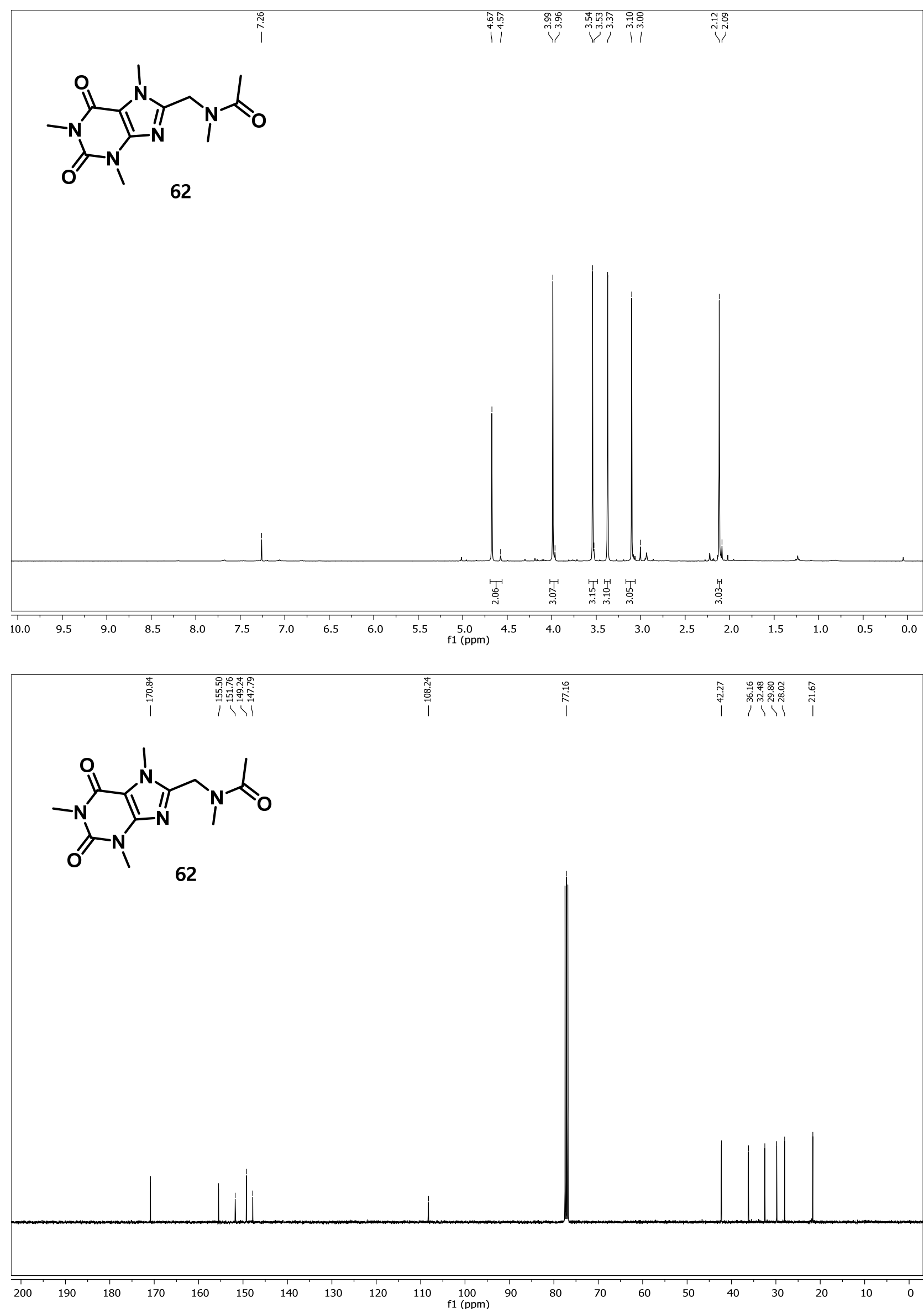

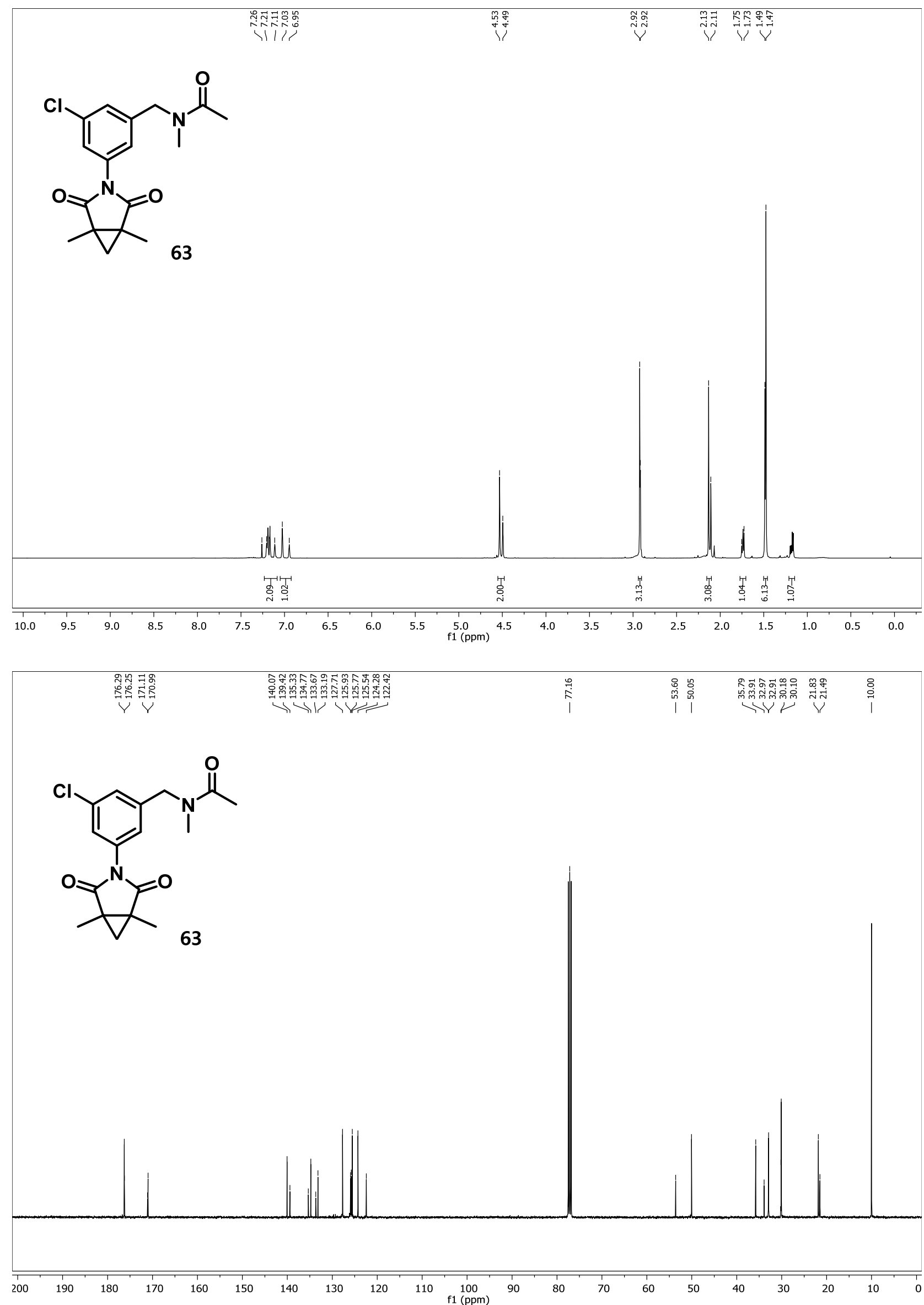

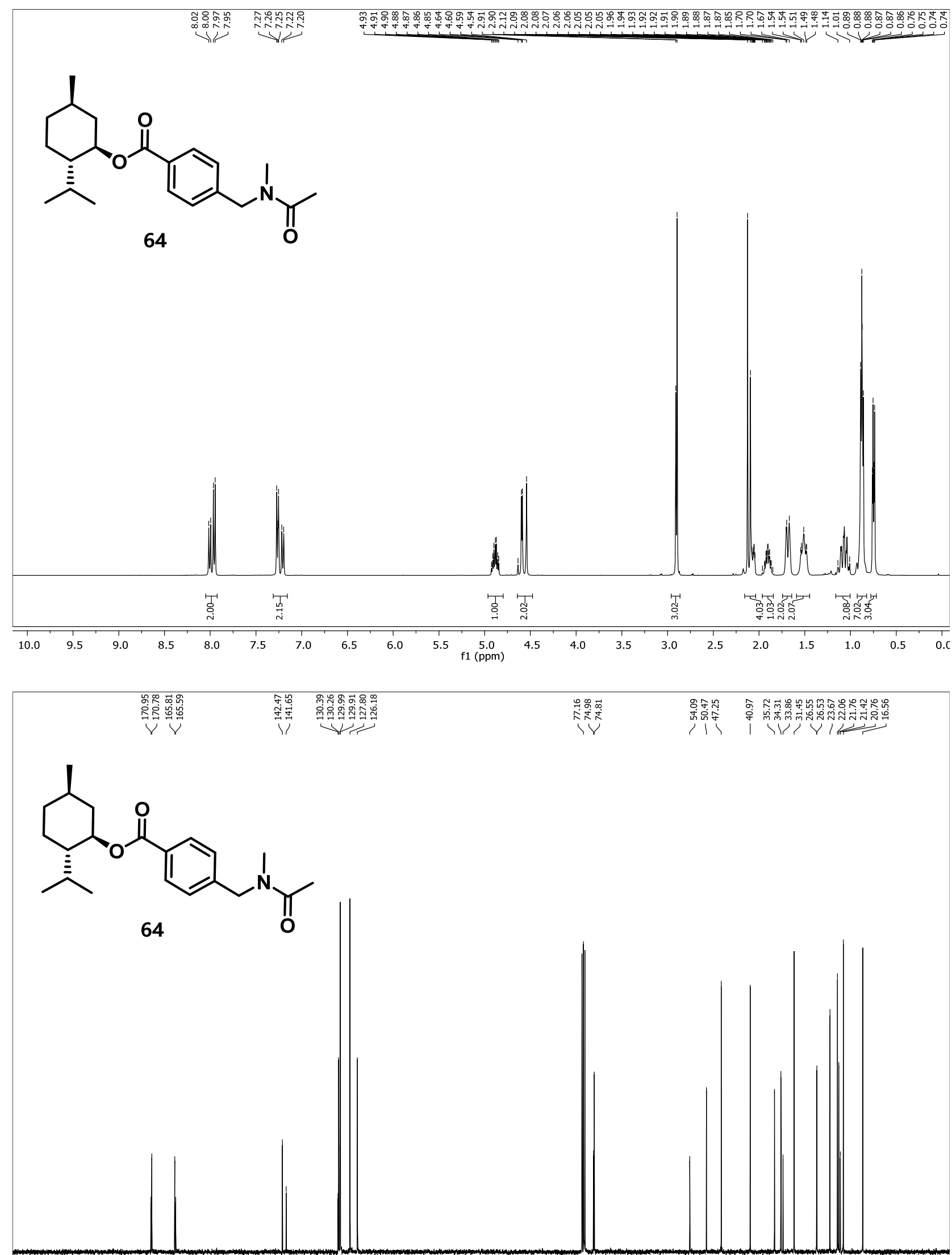

200

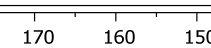

100
$1(\mathrm{ppm})$ 

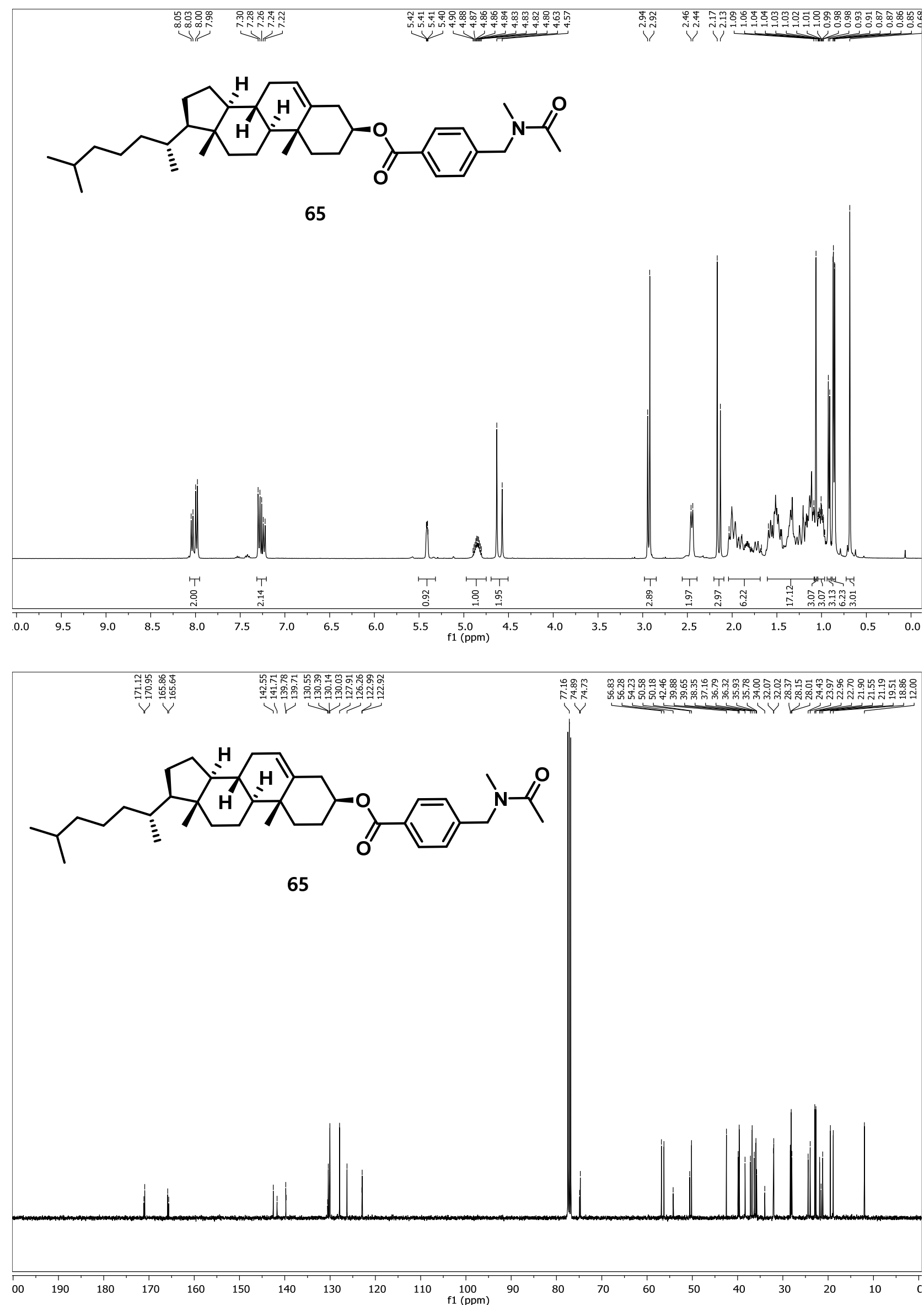

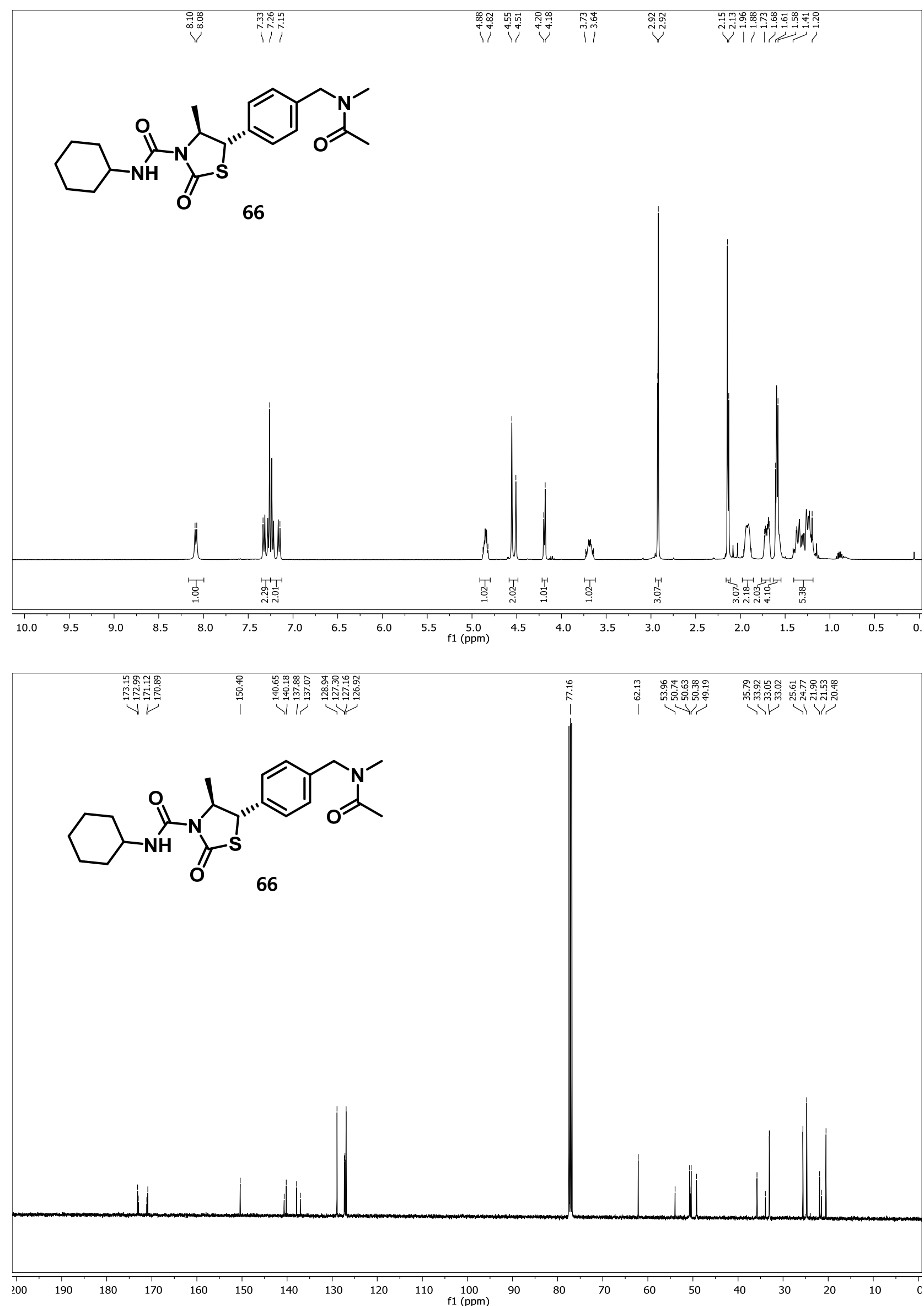

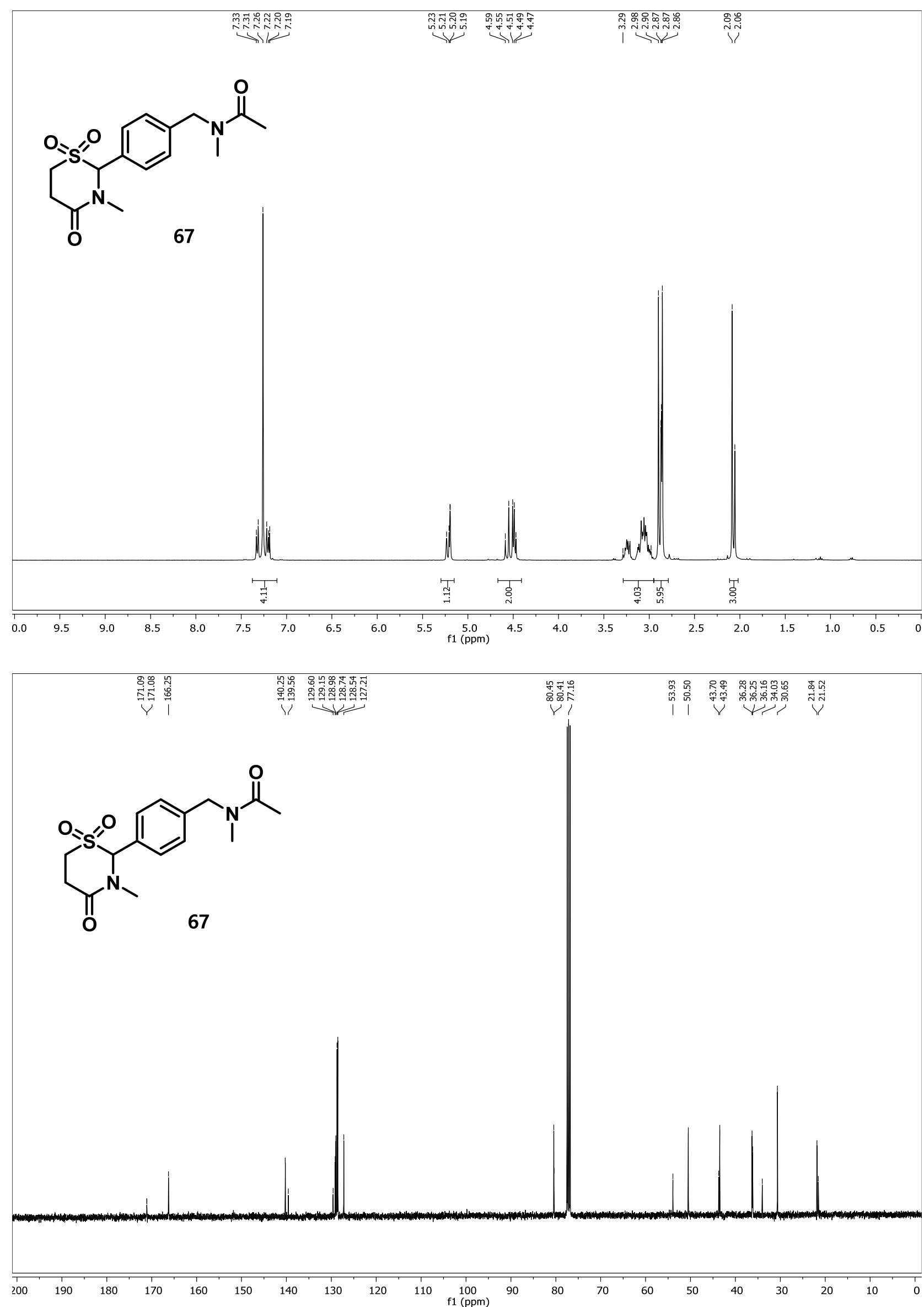

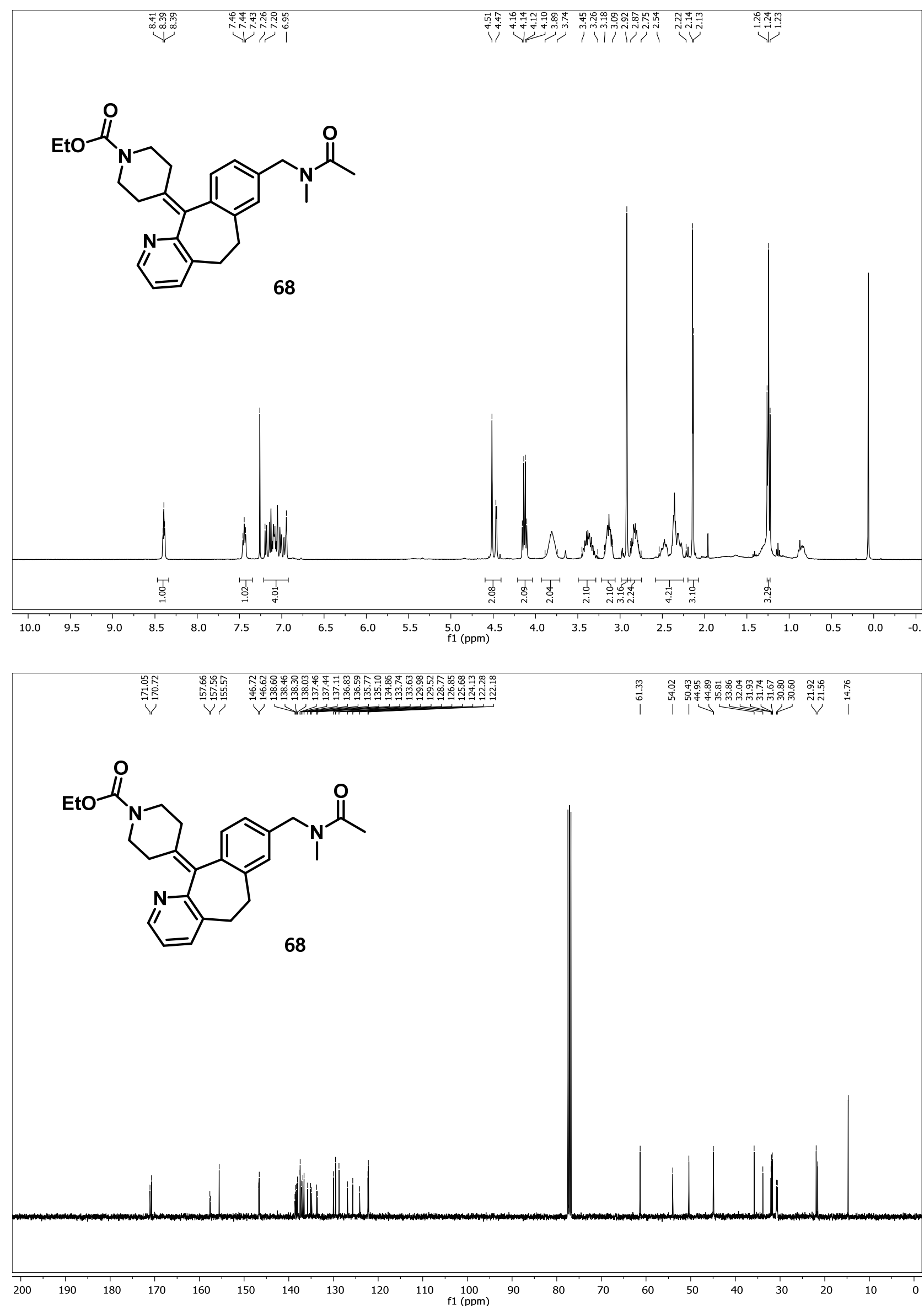

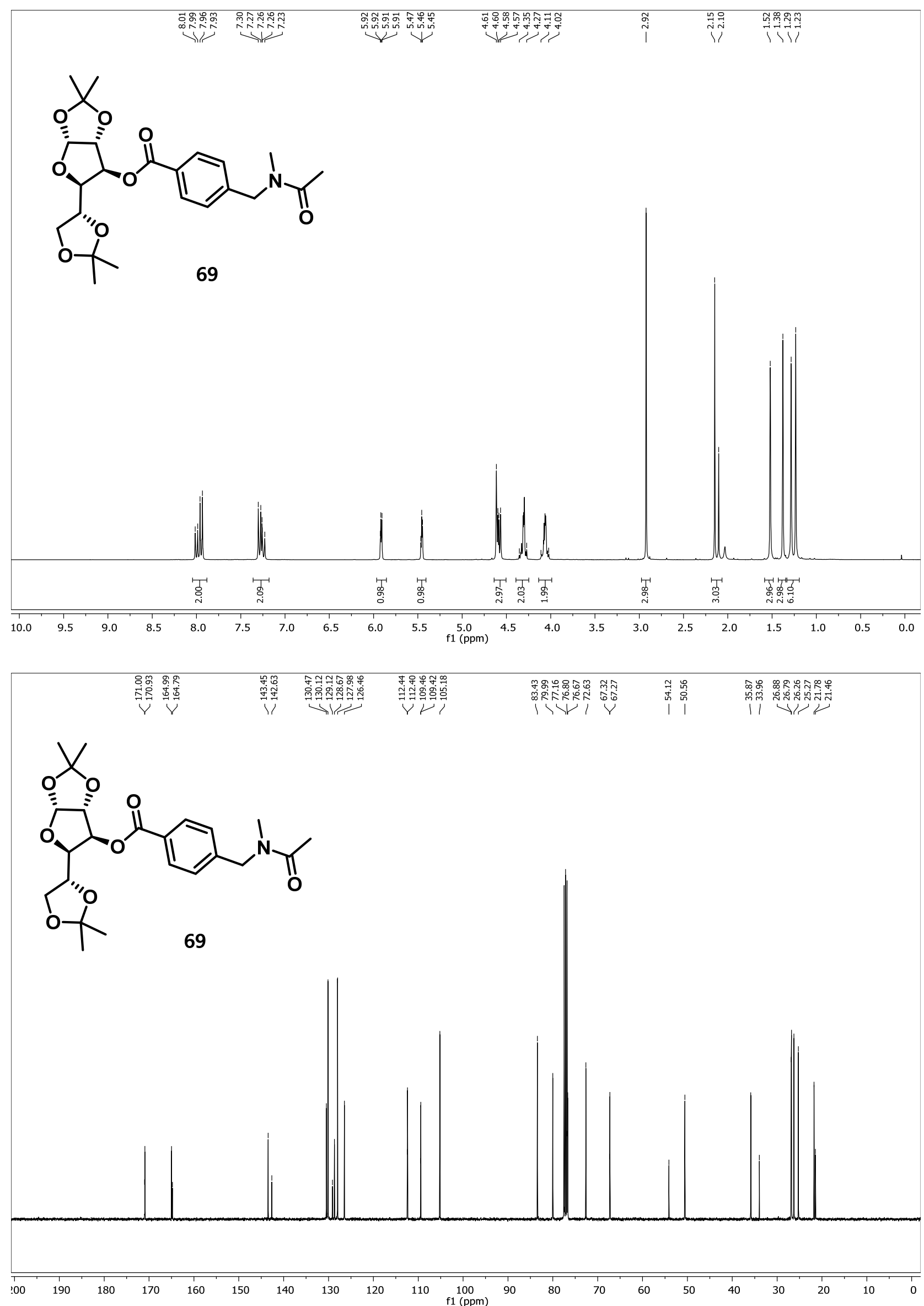

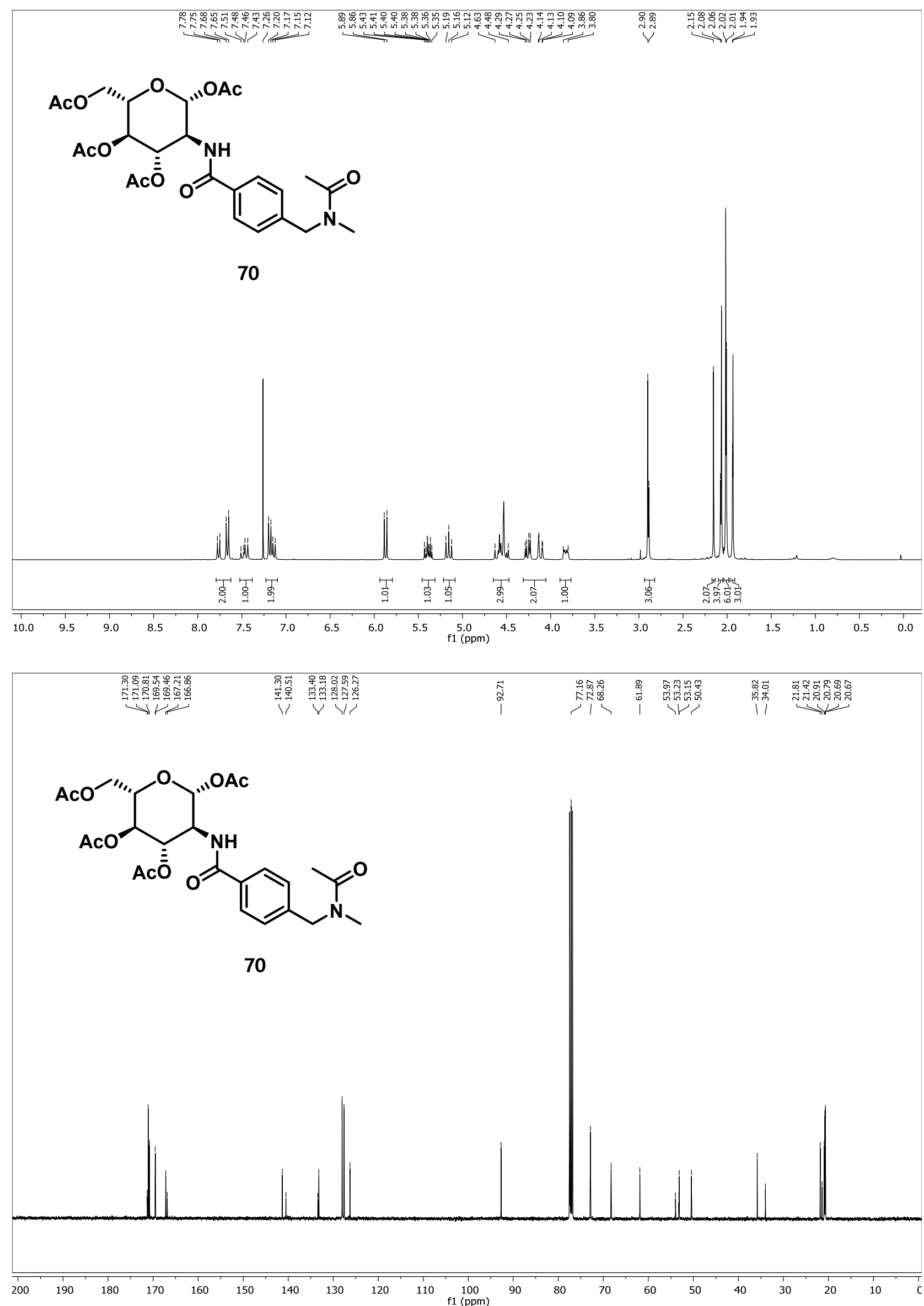

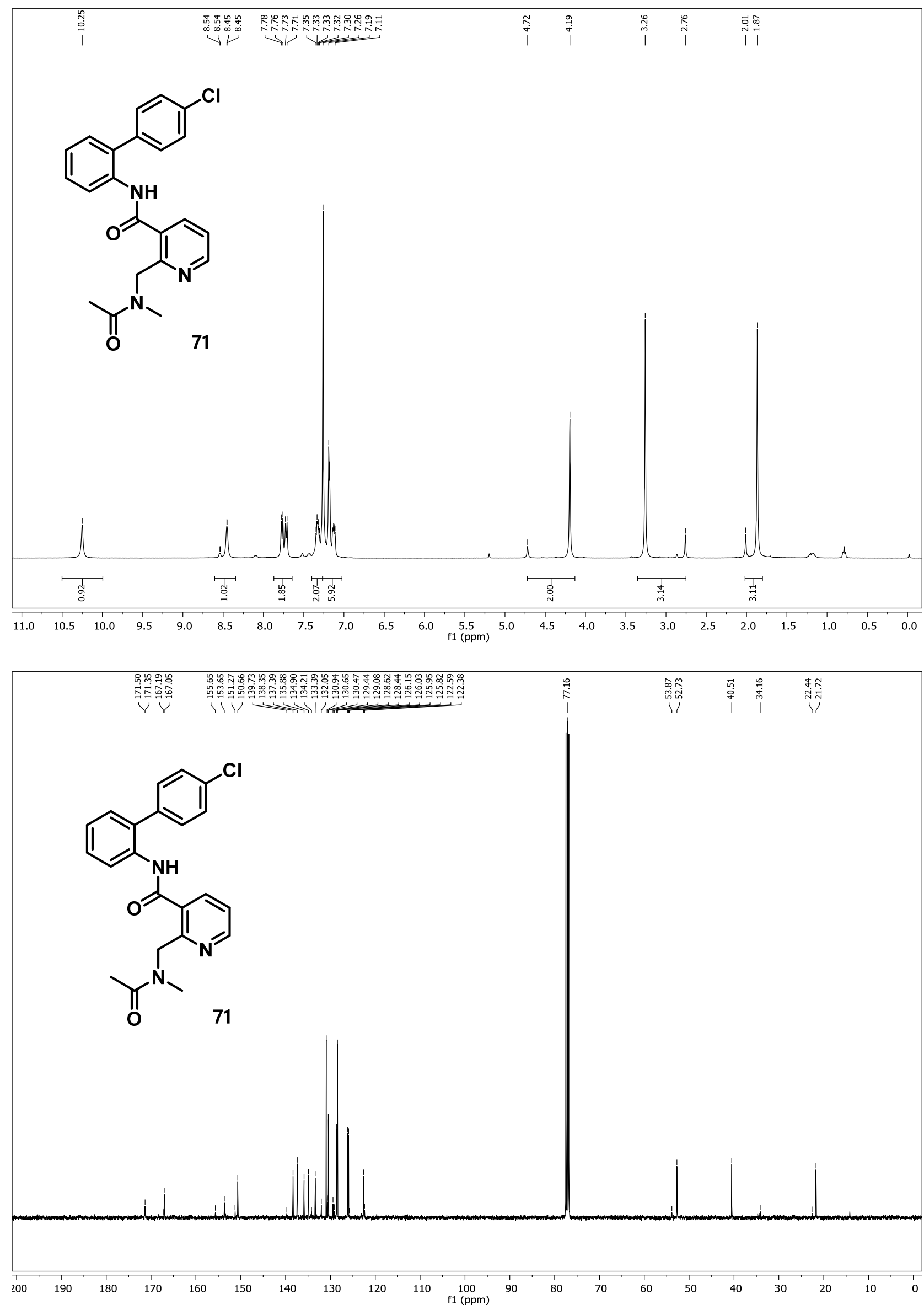

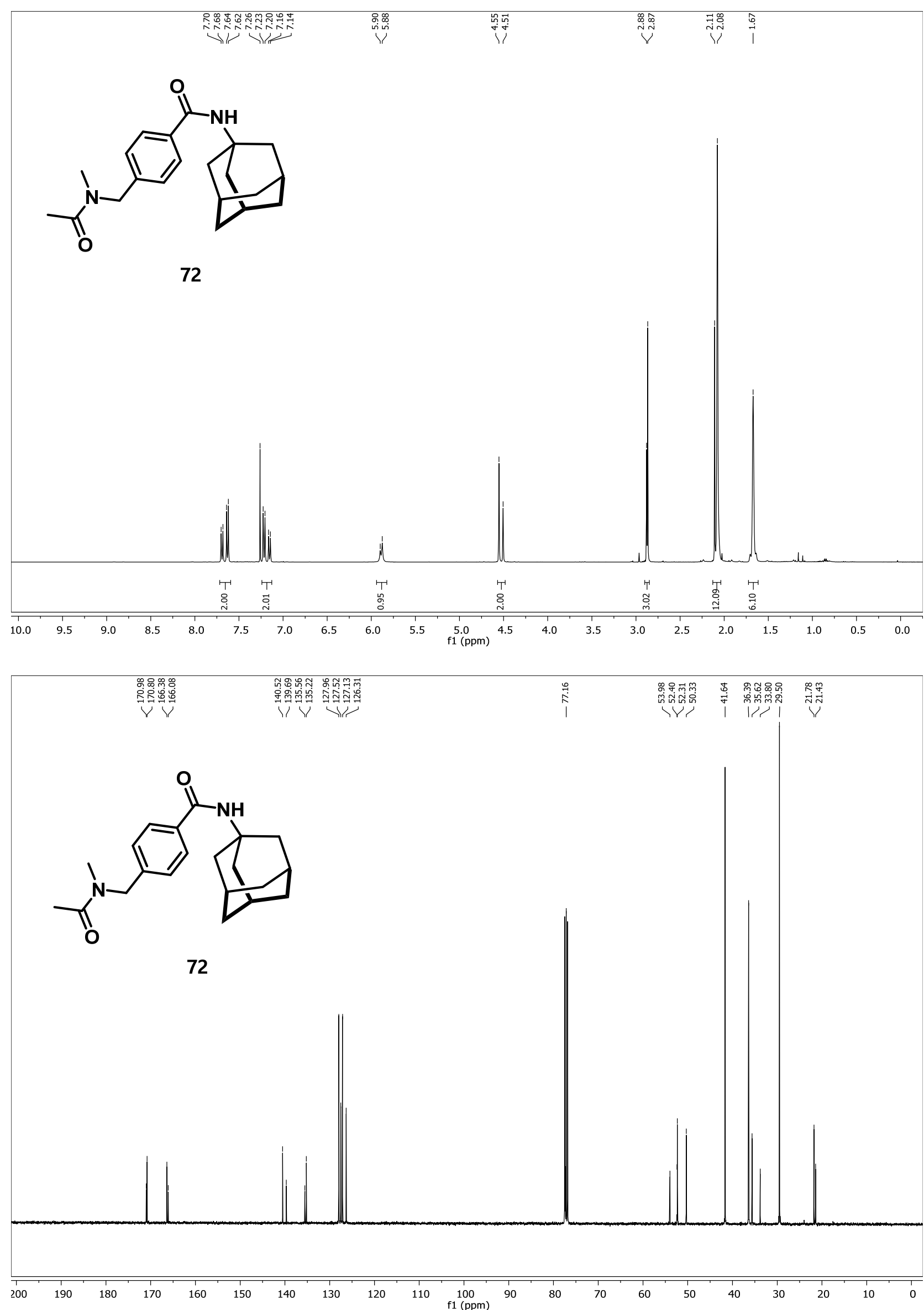

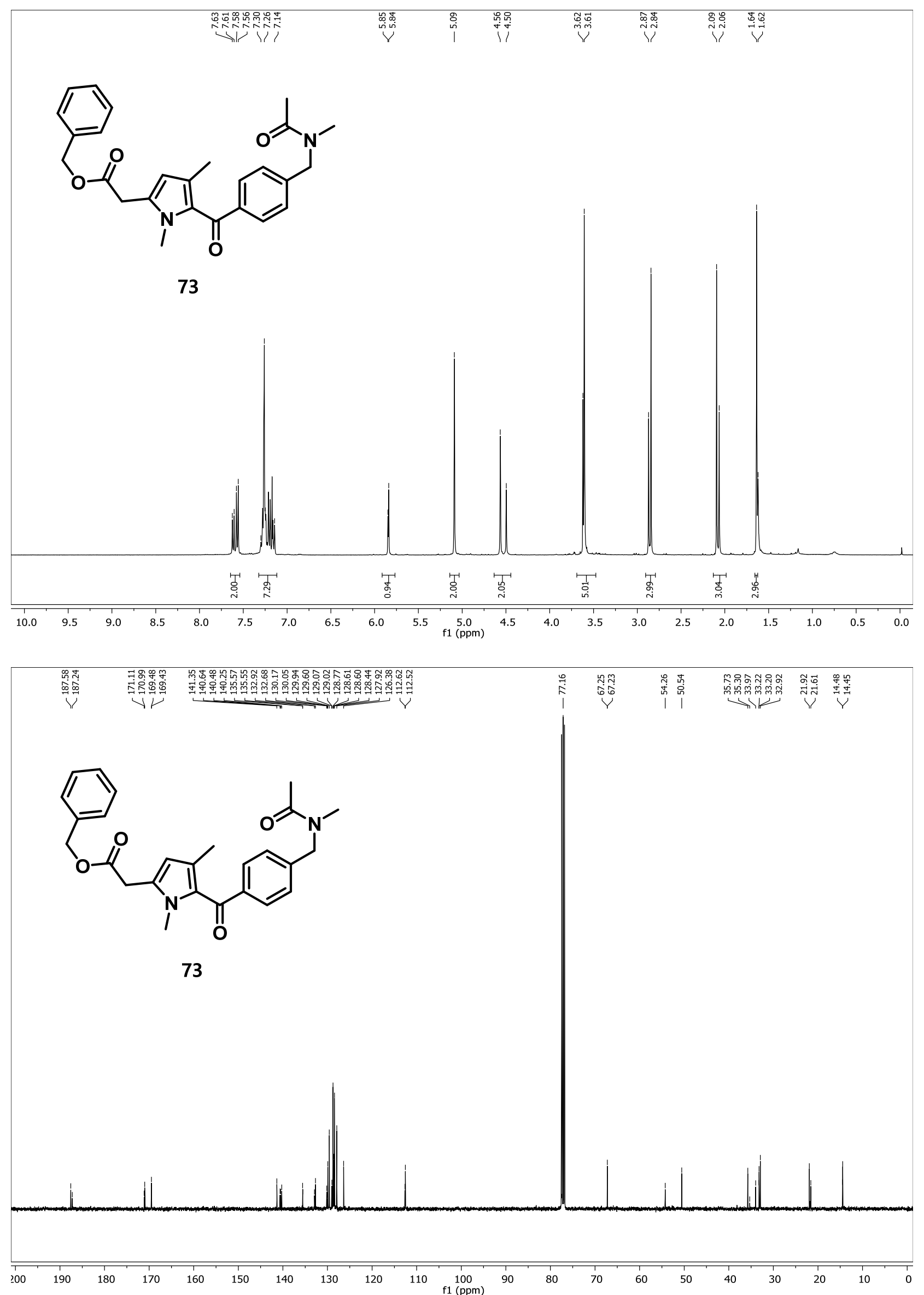

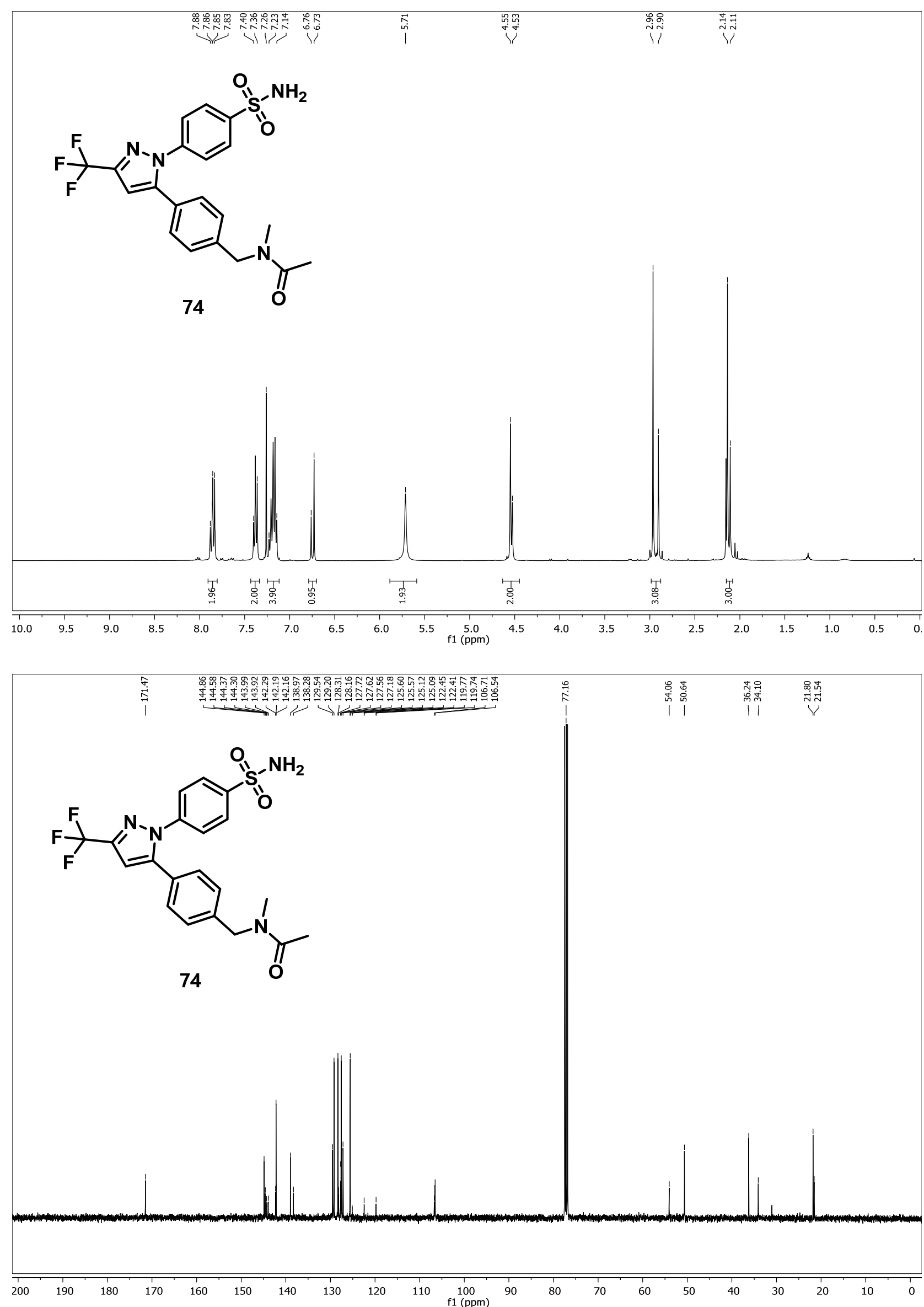

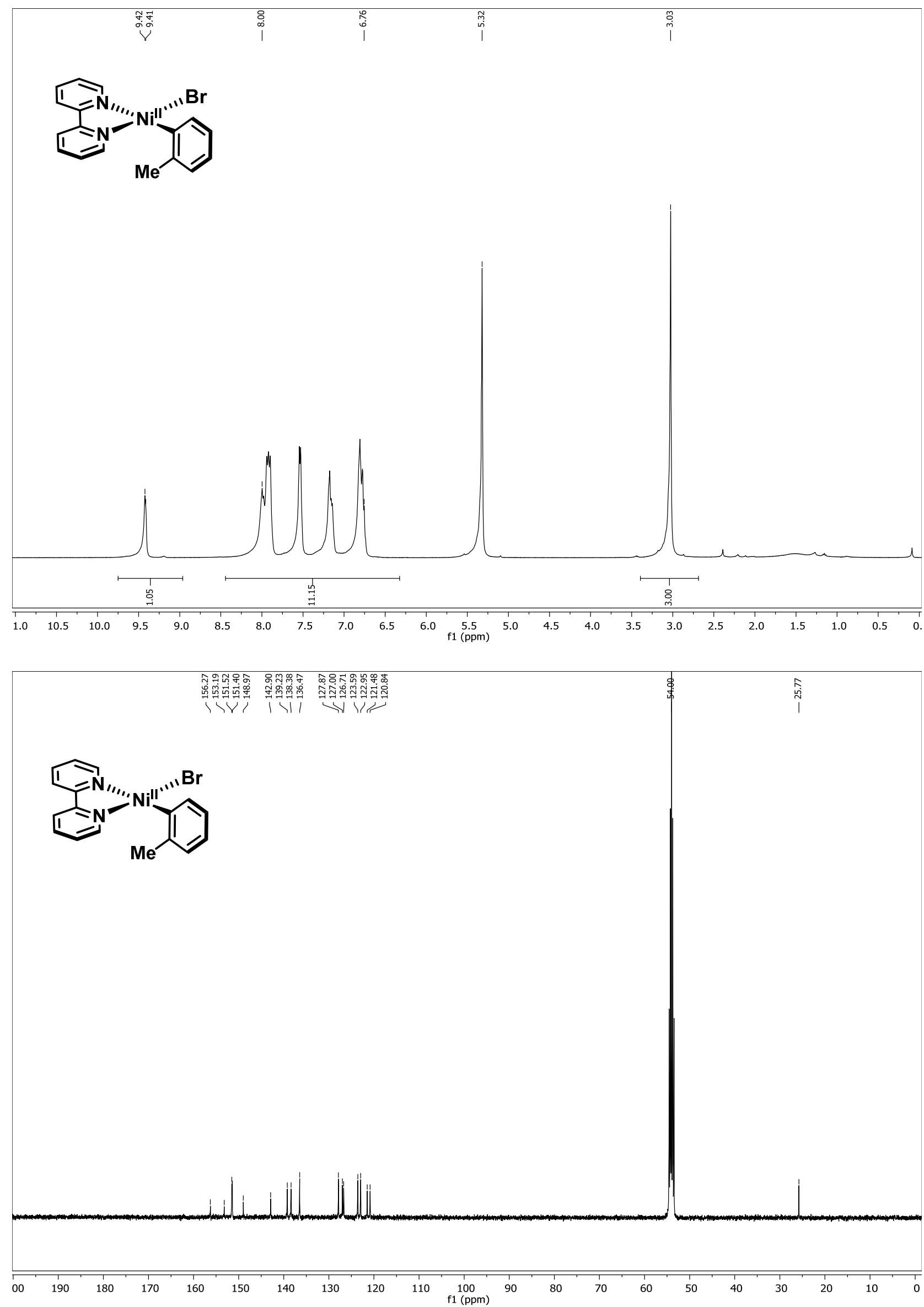